Supporting Information for

\title{
Diaza-Crown Ether-Bridged Chiral Diphosphoramidite Ligands: Synthesis and Applications in Asymmetric Catalysis
}

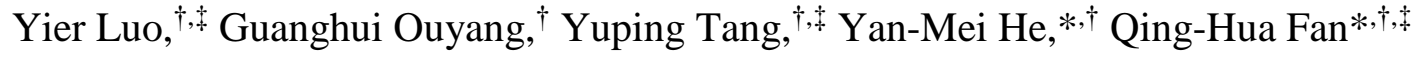 \\ 'Beijing National Laboratory for Molecular Sciences, CAS Key Laboratory of Molecular Recognition \\ and Function, Institute of Chemistry, Chinese Academy of Sciences (CAS), Beijing 100190 (P. R. \\ China) \\ University of Chinese Academy of Sciences, Beijing 100049 (P. R. China) \\ E-mail: fanqh@iccas.ac.cn; heym@iccas.ac.cn
}

\section{Contents}

1. Optimization of conditions for asymmetric reactions ----------------------------S2

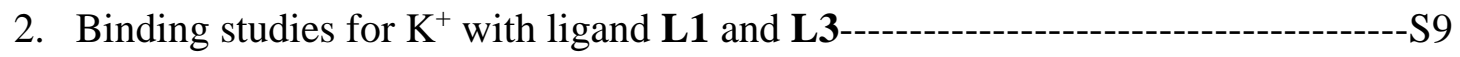

3. References--------------------------------------------------------------------------------S14

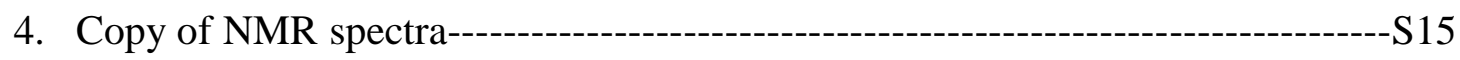

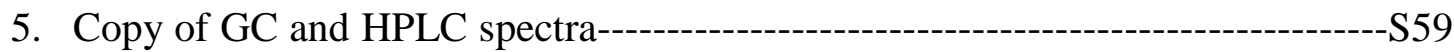




\section{Optimization of conditions for asymmetric reactions}

(a) Asymmetric hydrogenation of $\alpha$-dehydroamino acid esters

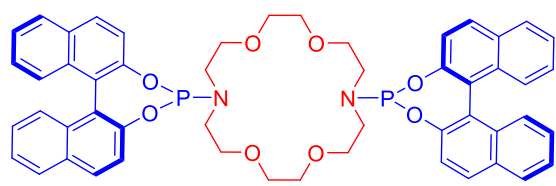

L1

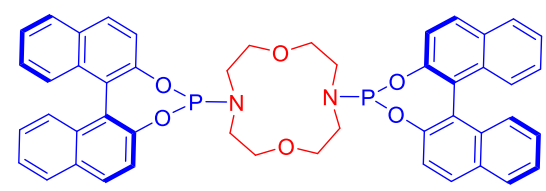

L4
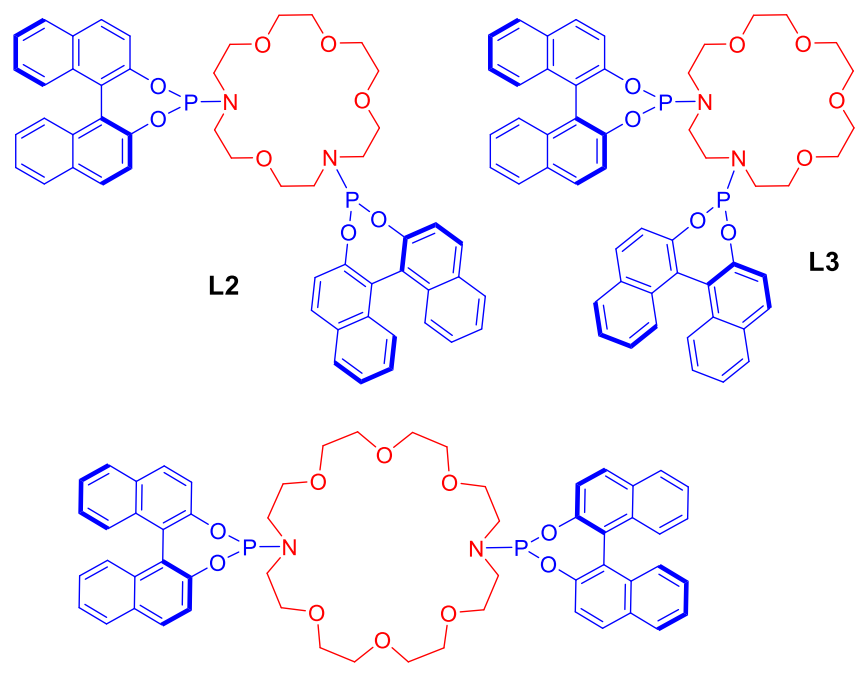

L5

Table $S 1$. Optimization of reaction solvent ${ }^{\mathrm{a}}$

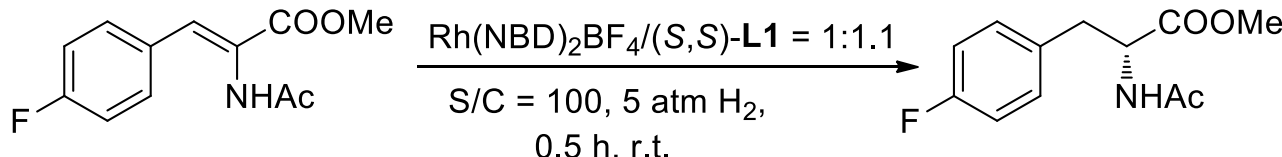

\begin{tabular}{cccc}
\hline Entry & Solvent & Conversion $(\%)^{\mathbf{b}}$ & Ee $(\%)^{\mathbf{b}}$ \\
\hline 1 & $\mathrm{MeOH}$ & $<5$ & -- \\
2 & $\mathrm{EtOH}$ & 9 & 96 \\
3 & $\mathrm{DCM}$ & $>99$ & $>99$ \\
4 & $\mathrm{THF}$ & 94 & 98 \\
5 & $\mathrm{Et}_{2} \mathrm{O}^{\mathrm{c}}$ & 12 & 97 \\
6 & acetone & 8 & 94 \\
7 & EtOAc $^{\mathrm{c}}$ & 1.1 & -- \\
8 & toluene $^{\mathrm{c}}$ & 22 & $>99$
\end{tabular}

${ }^{a}$ Reaction conditions: $\operatorname{Rh}(\mathrm{NBD}){ }_{2} \mathrm{BF}_{4}(1.0 \mathrm{~mol} \%),(S, S)-\mathbf{L 1}(1.1 \mathrm{~mol} \%)$, substrate $(0.05 \mathrm{mmol})$,

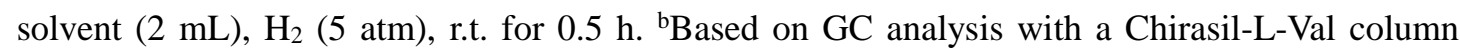
CP7495 (25 m×0.25 mm). ${ }^{c}$ Suspension solution. DCM = dichloromethane. 
Table $S 2$. Optimization of $\mathrm{H}_{2}$ pressure $^{\mathrm{a}}$

COOMe $\frac{\mathrm{Rh}(\mathrm{NBD})_{2} \mathrm{BF}_{4} /(\mathrm{S}, \mathrm{S})-\mathrm{L1}=1: 1.1}{\mathrm{~S} / \mathrm{C}=100, \mathrm{DCM}, 0.5 \mathrm{~h} \text {, r.t. }}$

\begin{tabular}{cccc}
\hline Entry & $\mathbf{H}_{\mathbf{2}}(\mathbf{a t m})$ & Conversion $(\boldsymbol{\%})^{\mathbf{b}}$ & $\mathbf{E e}(\boldsymbol{\%})^{\mathbf{b}}$ \\
\hline 1 & 1 & 30 & $>99$ \\
2 & 5 & $>99$ & $>99$ \\
3 & 10 & $>99$ & $>99$ \\
4 & 30 & $>99$ & $>99$ \\
5 & 50 & $>99$ & $>99$ \\
\hline
\end{tabular}

${ }^{a}$ Reaction conditions: $\mathrm{Rh}(\mathrm{NBD}){ }_{2} \mathrm{BF}_{4}(1.0 \mathrm{~mol} \%),(S, S)-\mathbf{L 1}(1.1 \mathrm{~mol} \%)$, Substrate: $0.05 \mathrm{mmol}$, DCM ( $2 \mathrm{~mL}), \mathrm{H}_{2}$, r.t. for 0.5 h. ${ }^{b}$ Based on GC analysis with a Chirasil-L-Val column CP7495 (25 $\mathrm{m} \times 0.25 \mathrm{~mm})$.

Table S3. Optimization of reaction temperature ${ }^{\mathrm{a}}$

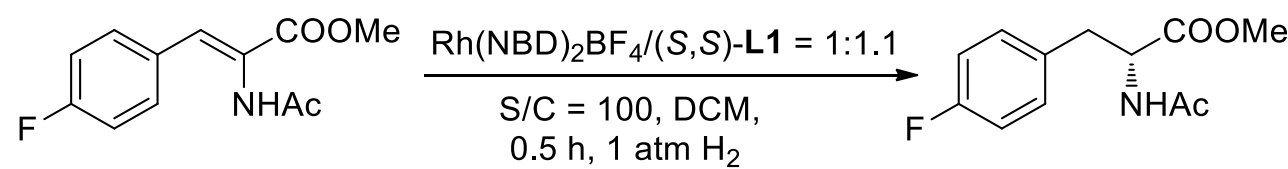

\begin{tabular}{cccc}
\hline Entry & ${\text { Temperature }\left({ }^{\circ} \mathbf{C}\right)}$ & Conversion $(\%)^{\mathbf{b}}$ & $\mathbf{E e}(\%)^{\mathbf{b}}$ \\
\hline 1 & 0 & 20 & $>99$ \\
2 & 25 & 30 & $>99$ \\
3 & 55 & 51 & 98 \\
\hline
\end{tabular}

${ }^{\mathrm{a}}$ Reaction conditions: $\mathrm{Rh}(\mathrm{NBD}){ }_{2} \mathrm{BF}_{4}(1.0 \mathrm{~mol} \%),(S, S)-\mathbf{L 1}(1.1 \mathrm{~mol} \%)$, substrate $(0.05 \mathrm{mmol})$, DCM (2 mL), $\mathrm{H}_{2}(1 \mathrm{~atm}), 0.5$ h. ${ }^{\mathrm{b}}$ Based on GC analysis with a Chirasil-L-Val column CP7495 (25 $\mathrm{m} \times 0.25 \mathrm{~mm})$. 
Table S4. Optimization of catalyst loadinga

COOMe $\frac{\mathrm{Rh}(\mathrm{NBD})_{2} \mathrm{BF}_{4} /(\mathrm{S}, S)-\mathbf{L 1}=1: 1.1}{\mathrm{DCM}, 1 \mathrm{~h}, 1 \mathrm{~atm} \mathrm{H} \mathrm{H}_{2} \text {, r.t. }} \mathrm{COOMe}$

\begin{tabular}{cccc}
\hline Entry & Substrate/Catalyst & ${\text { Conversion }(\%)^{\mathbf{b}}}$ & Ee (\%) \\
\hline 1 & 100 & 70 & $>99$ \\
2 & 300 & 11 & 98 \\
3 & 500 & 7.3 & 97 \\
4 & 1000 & 1.8 & 93 \\
$5^{\mathrm{c}}$ & 500 & 94 & 97 \\
\hline
\end{tabular}

${ }^{a}$ Reaction conditions: $\mathrm{Rh}(\mathrm{NBD})_{2} \mathrm{BF}_{4},(S, S)-\mathbf{L 1}$, substrate $(0.05 \mathrm{mmol}), \mathrm{DCM}(2 \mathrm{~mL}), \mathrm{H}_{2}(1 \mathrm{~atm})$, r.t. for $1 \mathrm{~h} .{ }^{b}$ Based on $\mathrm{GC}$ analysis with a Chirasil-L-Val column CP7495 (25 $\left.\mathrm{m} \times 0.25 \mathrm{~mm}\right) .{ }^{\mathrm{c}} \mathrm{H}_{2}$ (50 atm), $21 \mathrm{~h}$.

Table $S 5$. Screening of chiral ligand ${ }^{\mathrm{a}}$

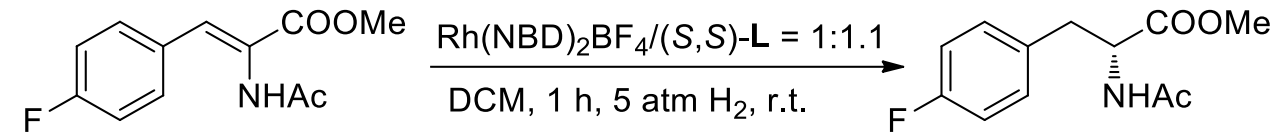

\begin{tabular}{cccc}
\hline Entry & Ligand & Conversion $(\boldsymbol{\%})^{\mathbf{b}}$ & Ee $(\boldsymbol{\%})^{\mathbf{b}}$ \\
\hline 1 & $\mathbf{L 1}$ & $>99$ & $>99(R)$ \\
2 & $\mathbf{L 2}$ & $<5$ & -- \\
3 & $\mathbf{L 3}$ & $>99$ & $94(S)$ \\
4 & $\mathbf{L 4}$ & $>99$ & $98(R)$ \\
$5^{\mathrm{c}}$ & $\mathbf{L 5}$ & 39 & $84(R)$ \\
\hline
\end{tabular}

${ }^{\mathrm{a}}$ Reaction conditions: $\mathrm{Rh}(\mathrm{NBD}){ }_{2} \mathrm{BF}_{4}(1.0 \mathrm{~mol} \%),(S, S)-\mathbf{L 1}(1.1 \mathrm{~mol} \%)$, substrate $(0.05 \mathrm{mmol})$, DCM $(2 \mathrm{~mL}), \mathrm{H}_{2}(1 \mathrm{~atm})$, r.t. for $1 \mathrm{~h} .{ }^{\mathrm{b}}$ Based on GC analysis with a Chirasil-L-Val column CP7495 (25 m×0.25 mm). ${ }^{\mathrm{c}} \mathrm{H}_{2}(50 \mathrm{~atm}), 21 \mathrm{~h}$. 


\section{(b) Asymmetric hydrogenation of enamides}

\section{Table S6. Optimization of reaction solvent ${ }^{\mathrm{a}}$}

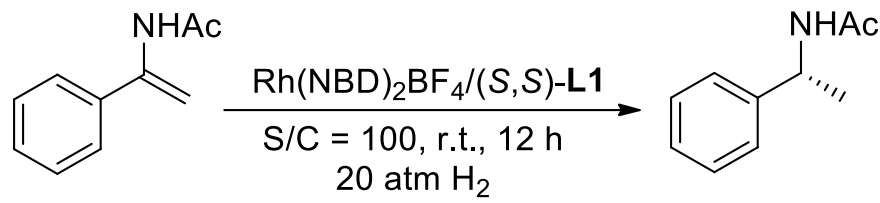

\begin{tabular}{cccc}
\hline Entry & Solvent & Conversion $(\boldsymbol{\%})^{\mathbf{b}}$ & Ee (\%) \\
\hline 1 & MeOH & 12 & 25 \\
2 & ${ }^{\mathbf{c}} \mathrm{PrOH}$ & 30 & 76 \\
3 & ${ }^{\mathrm{BuOH}}$ & 19 & 92 \\
4 & $\mathrm{DCM}$ & $>99$ & 86 \\
5 & $\mathrm{THF}$ & 47 & 68 \\
$6^{\mathrm{d}}$ & $\mathrm{EtOAc}$ & 8 & 75 \\
$7^{\mathrm{d}}$ & $n$-hexane & 49 & 98 \\
$8^{\mathrm{d}}$ & toluene & 42 & 97 \\
9 & $n$-hexane $:$ DCM $=1: 1$ & $>99$ & 97 \\
10 & $n$-hexane $:$ DCM $=1: 2$ & 89 & 95 \\
11 & $n$-hexane $:$ DCM $=1: 3$ & 81 & 96 \\
12 & $n$-hexane $:$ DCM $=1: 5$ & 62 & 97
\end{tabular}

${ }^{a}$ Reaction conditions: $\operatorname{Rh}(\mathrm{NBD}){ }_{2} \mathrm{BF}_{4}(1.0 \mathrm{~mol} \%),(S, S)-\mathbf{L 1}(1.1 \mathrm{~mol} \%)$, substrate $(0.05 \mathrm{mmol})$, solvent $(2 \mathrm{~mL}), \mathrm{H}_{2}(20 \mathrm{~atm})$, r.t. for $12 \mathrm{~h} .{ }^{\mathrm{b}}$ The conversion were determined by ${ }^{1} \mathrm{H}$ NMR. ${ }^{\mathrm{c}}$ Based on GC analysis with a chrompack Chirasil-Dex CB column CP7502 (25 $\mathrm{m} \times 0.25 \mathrm{~mm}){ }^{\mathrm{d}}$ Suspension solution. 
Table S7. Optimization of $\mathrm{H}_{2}$ pressure ${ }^{\mathrm{a}}$

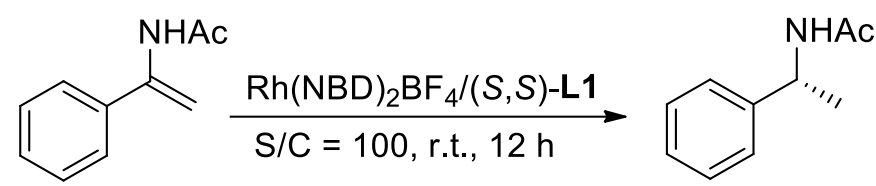

\begin{tabular}{cccc}
\hline Entry & $\mathbf{H}_{\mathbf{2}}(\mathbf{a t m})$ & ${\text { Conversion }(\boldsymbol{\%})^{\mathbf{b}}}$ & Ee (\%) \\
\hline 1 & 1 & 16 & 93 \\
2 & 5 & 67 & 92 \\
3 & 10 & 91 & 96 \\
4 & 15 & 95 & 96 \\
5 & 20 & $>99$ & 97
\end{tabular}

${ }^{a}$ Reaction conditions: $\operatorname{Rh}(\mathrm{NBD})_{2} \mathrm{BF}_{4}(1.0 \mathrm{~mol} \%),(S, S)-\mathbf{L 1}(1.1 \mathrm{~mol} \%)$, substrate $(0.05 \mathrm{mmol})$, $n$-hexane : DCM $=1: 1(2 \mathrm{~mL}), \mathrm{H}_{2}$, r.t. for $12 \mathrm{~h}$. ${ }^{\mathrm{b}}$ The conversions were determined by ${ }^{1} \mathrm{H}$ NMR. ${ }^{\mathrm{c}}$ Based on GC analysis with a chrompack Chirasil-Dex CB column CP7502 (25 m×0.25 mm).

Table $S 8$. Optimization of reaction temperature ${ }^{\mathrm{a}}$

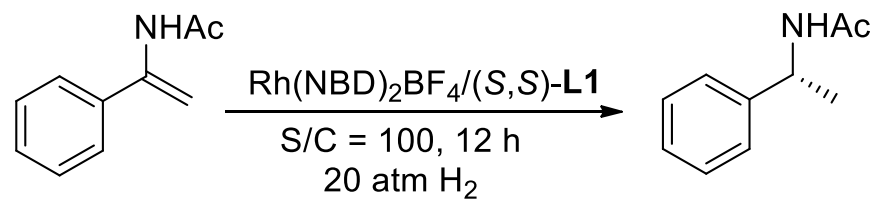

\begin{tabular}{cccc}
\hline Entry & Temperature $\left({ }^{\circ} \mathbf{C}\right)$ & Conversion $(\%)^{\mathbf{b}}$ & $\operatorname{Ee~}(\%)^{\mathbf{c}}$ \\
\hline 1 & 0 & 87 & 97 \\
2 & 25 & $>99$ & 97 \\
3 & 50 & $>99$ & 89 \\
\hline
\end{tabular}

${ }^{\mathrm{a}}$ Reaction conditions: $\mathrm{Rh}(\mathrm{NBD}){ }_{2} \mathrm{BF}_{4}(1.0 \mathrm{~mol} \%),(S, S)-\mathbf{L 1}(1.1 \mathrm{~mol} \%)$, substrate $(0.05 \mathrm{mmol})$, $n$-hexane : $\mathrm{DCM}=1: 1(2 \mathrm{~mL}), \mathrm{H}_{2}(20 \mathrm{~atm}), 12 \mathrm{~h} .{ }^{\mathrm{b}}$ The conversions were determined by ${ }^{1} \mathrm{H}$ NMR.

${ }^{\mathrm{c}}$ Based on GC analysis with a chrompack Chirasil-Dex CB column CP7502 (25 m×0.25 mm). 
Table $S 9$. Screening of ligand

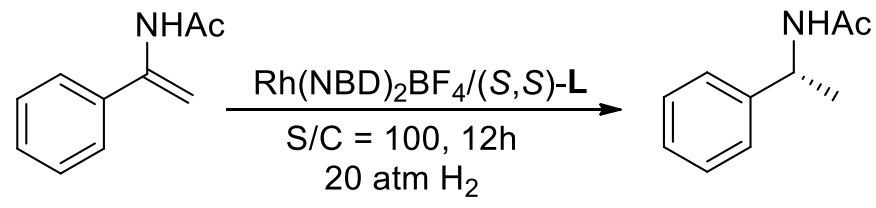

\begin{tabular}{|c|c|c|c|c|}
\hline Entry & Ligand & Additive (mol \%) & Conversion $(\%)^{\mathbf{b}}$ & $\operatorname{Ee}(\%)^{\mathrm{c}}$ \\
\hline 1 & $(S, S)-\mathbf{L} \mathbf{1}$ & None & $>99$ & $97(R)$ \\
\hline 2 & $(S, S)-\mathbf{L} \mathbf{2}$ & None & $<5$ & -- \\
\hline 3 & $(S, S)-\mathbf{L} \mathbf{3}$ & None & $>99$ & $86(S)$ \\
\hline 4 & $(S, S)-\mathbf{L} \mathbf{4}$ & None & 44 & $85(R)$ \\
\hline 5 & $(S, S)-\mathbf{L} \mathbf{5}$ & None & $>99$ & $72(S)$ \\
\hline 6 & $(S, S)-\mathbf{L} \mathbf{1}$ & $\operatorname{KB} \operatorname{ArF}(1.1)$ & $>99$ & $41(R)$ \\
\hline 7 & $(S, S)-\mathbf{L} \mathbf{3}$ & $\operatorname{KBArF}(1.1)$ & $>99$ & $91(S)$ \\
\hline 8 & $(S, S)-\mathbf{L} \mathbf{3}$ & $\begin{array}{c}\text { KBArF (1.1)/ } \\
\text { Kryptofix } 222(1.1)\end{array}$ & $>99$ & $86(S)$ \\
\hline
\end{tabular}

${ }^{\mathrm{a}}$ Reaction conditions: $\mathrm{Rh}(\mathrm{NBD})_{2} \mathrm{BF}_{4}(1.0 \mathrm{~mol} \%),(S, S)-\mathrm{L}(1.1 \mathrm{~mol} \%)$, substrate $(0.05 \mathrm{mmol})$, $n$-hexane $: \mathrm{DCM}=1: 1(2 \mathrm{~mL}), \mathrm{H}_{2}(20 \mathrm{~atm})$, r.t. for $12 \mathrm{~h} .{ }^{\mathrm{b}}$ The conversions were determined by ${ }^{1} \mathrm{H}$ NMR. ${ }^{c}$ Based on GC analysis with a chrompack Chirasil-Dex CB column CP7502 (25 m×0.25 $\mathrm{mm})$. 
(c) Asymmetric hydroformylation of styrenes

Table S10. Optimization of reaction solvent ${ }^{\mathrm{a}}$

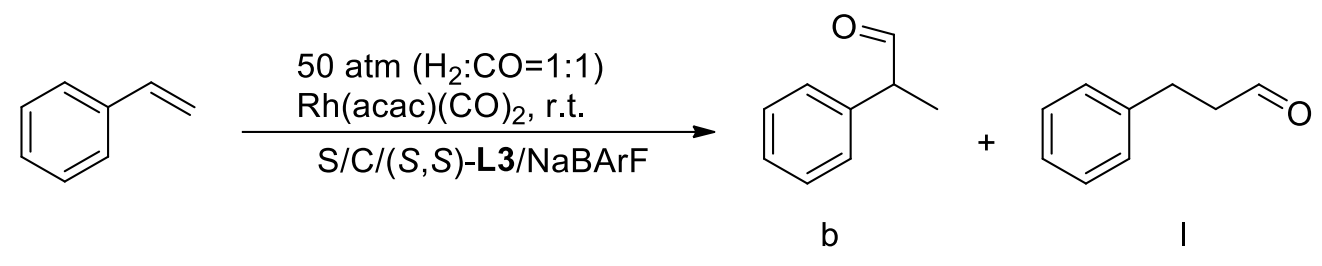

\begin{tabular}{|c|c|c|c|c|}
\hline Entry & Solvent & Conversion $(\%)^{\mathbf{b}}$ & b/l (Product) ${ }^{b}$ & $\operatorname{Ee}(\%)^{b}$ \\
\hline 1 & $\mathrm{EtOH}$ & 7 & $92: 8$ & 32 \\
\hline 2 & ${ }^{i} \mathrm{PrOH}$ & 8 & $92: 8$ & 35 \\
\hline 3 & THF & 6 & $94: 6$ & -13 \\
\hline 4 & DCM & 43 & $92: 8$ & -70 \\
\hline $5^{c}$ & $\mathrm{DCM}$ & 98 & $93: 7$ & -71 \\
\hline $6^{c}$ & $\mathrm{CDCl}_{3}$ & 90 & $93: 7$ & -70 \\
\hline $7^{c}$ & toluene & 80 & $93: 7$ & -67 \\
\hline $8^{c}$ & toluene & 80 & $93: 7$ & -67 \\
\hline
\end{tabular}

${ }^{a}$ Reaction conditions: $\operatorname{Rh}(\mathrm{acac})(\mathrm{CO})_{2}(1.0 \mathrm{~mol} \%),(S, S)-\mathbf{L 3}(1.0 \mathrm{~mol} \%), \mathrm{NaBArF}(1.0 \mathrm{~mol} \%)$, substrate $(0.1 \mathrm{mmol})$, solvent $(1 \mathrm{~mL})$, syngas $\left(\mathrm{H}_{2} / \mathrm{CO}=1: 1,50 \mathrm{~atm}\right)$, r.t. for $12 \mathrm{~h} .{ }^{\mathrm{b}}$ Based on $\mathrm{GC}$ analysis with a Supelco's Beta Dex 225 column $(30 \mathrm{~m} \times 0.25 \mathrm{~mm} \times 0.25 \mu \mathrm{m}) .{ }^{\mathrm{c}} \mathrm{Rh}(\mathrm{acac})(\mathrm{CO})_{2}(2.0$ mol \%), (S,S)-L3 (2.0 mol \%), NaBArF (2.0 mol \%), $24 \mathrm{~h}$. 
Table S11. Optimization of syngas $\left(\mathrm{H}_{2} / \mathrm{CO}=1: 1\right)$ pressure $^{\mathrm{a}}$

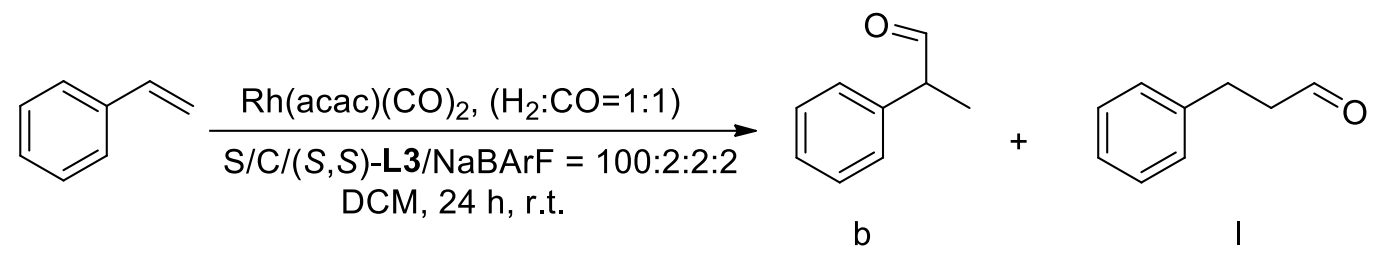

\begin{tabular}{ccccc}
\hline Entry & Pressure & Conversion $(\%)^{\mathbf{b}}$ & b/l (Product) & Ee $(\%)^{\mathbf{b}}$ \\
\hline 1 & $10 \mathrm{~atm}$ & $>99$ & $93: 7$ & -70 \\
2 & $20 \mathrm{~atm}$ & $>99$ & $93: 7$ & -70 \\
3 & $30 \mathrm{~atm}$ & 98 & $93: 7$ & -70 \\
4 & $50 \mathrm{~atm}$ & 98 & $93: 7$ & -71 \\
$5^{c}$ & $50 \mathrm{~atm}$ & $>99$ & $92: 8$ & -23 \\
\hline
\end{tabular}

${ }^{\text {a }}$ Reaction conditions: $\mathrm{Rh}(\mathrm{acac})(\mathrm{CO})_{2}(2.0 \mathrm{~mol} \%),(S, S)-\mathbf{L 3}(2.0 \mathrm{~mol} \%)$, NaBArF (2.0 mol \%), substrate $(0.1 \mathrm{mmol})$, DCM $(1 \mathrm{~mL})$, syngas $\left(\mathrm{H}_{2} / \mathrm{CO}=1: 1\right)$, r.t. for $24 \mathrm{~h} .{ }^{\mathrm{b}}$ Based on $\mathrm{GC}$ analysis with a Supelco's Beta Dex 225 column $(30 \mathrm{~m} \times 0.25 \mathrm{~mm} \times 0.25 \mu \mathrm{m}) .{ }^{\mathrm{c}} 50{ }^{\circ} \mathrm{C}$ for $5 \mathrm{~h}$.

\section{Binding studies for $\mathrm{K}^{+}$with ligand $\mathrm{L1}$ and $\mathbf{L 3}$}

The association of alkali metal cations with the synthesized ligands ( $\mathbf{L} \mathbf{1}$ and $\mathbf{L 3}$ ) was investigated. Upon the addition of KBArF, both ligands showed peaks shifts for both oxyethylene and aryl protons in ${ }^{1} \mathrm{H}$ NMR spectra and downfield shifts of the phosphoramidtes signals in ${ }^{31} \mathrm{P}$ NMR spectra, indicating the host-guest assembly occurred. Furthermore, a 1:1 guest-host $\left(\mathrm{K}^{+}-\mathrm{L} 3\right)$ ratio was determined by Job plot analysis ${ }^{[1 \mathrm{a}]}$, and the association constant calculated from the ${ }^{1} \mathrm{H}$ NMR spectroscopy titration data by linear data fitting ${ }^{[1 \mathrm{~b}]}$ was $33 \pm 1 \mathrm{M}^{-1}$. 
(1) ${ }^{1} \mathrm{H}$ NMR and ${ }^{31} \mathrm{P}$ NMR spectra for ligands $\mathrm{L1}$ and $\mathrm{L3}$ without and with KBArF
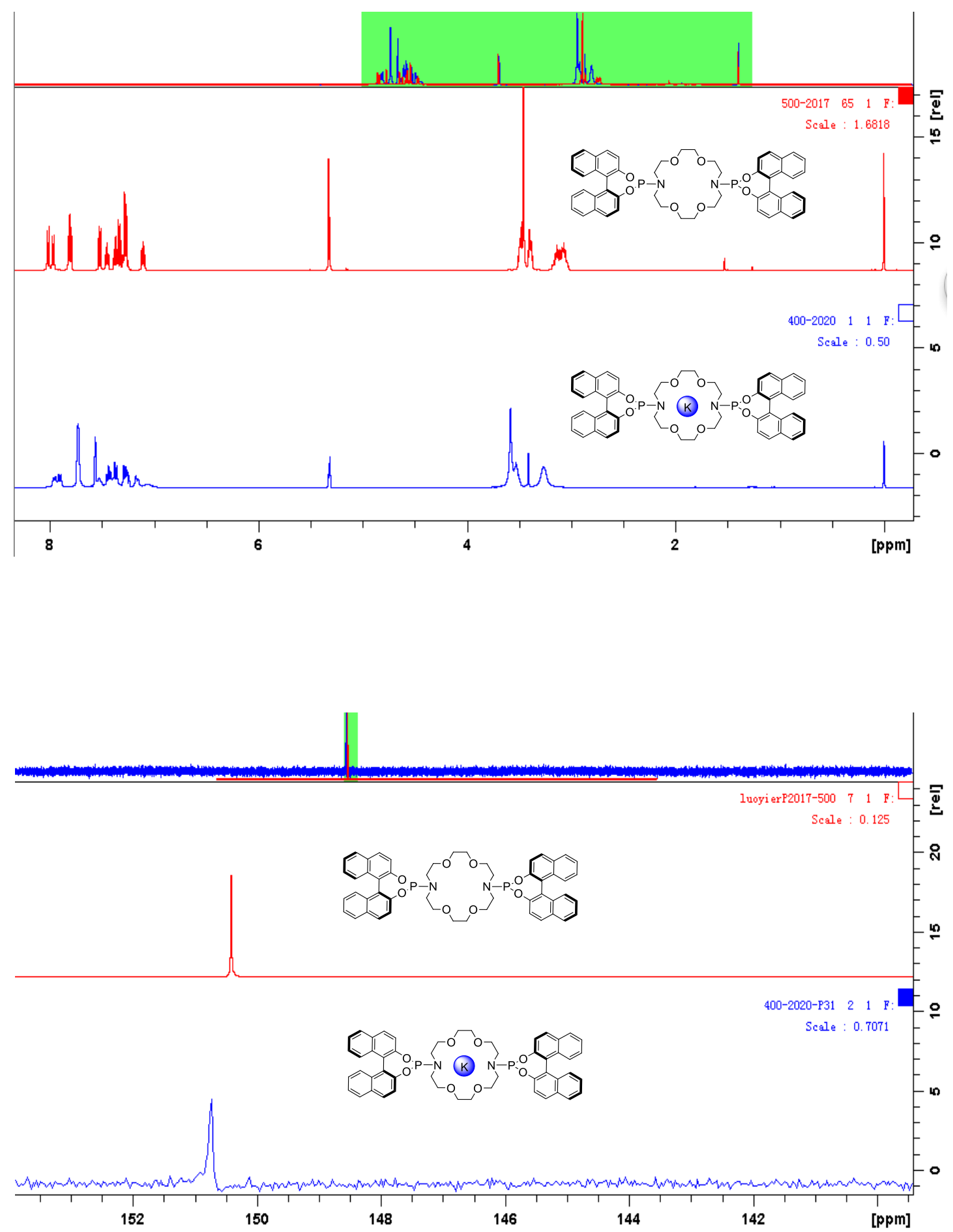

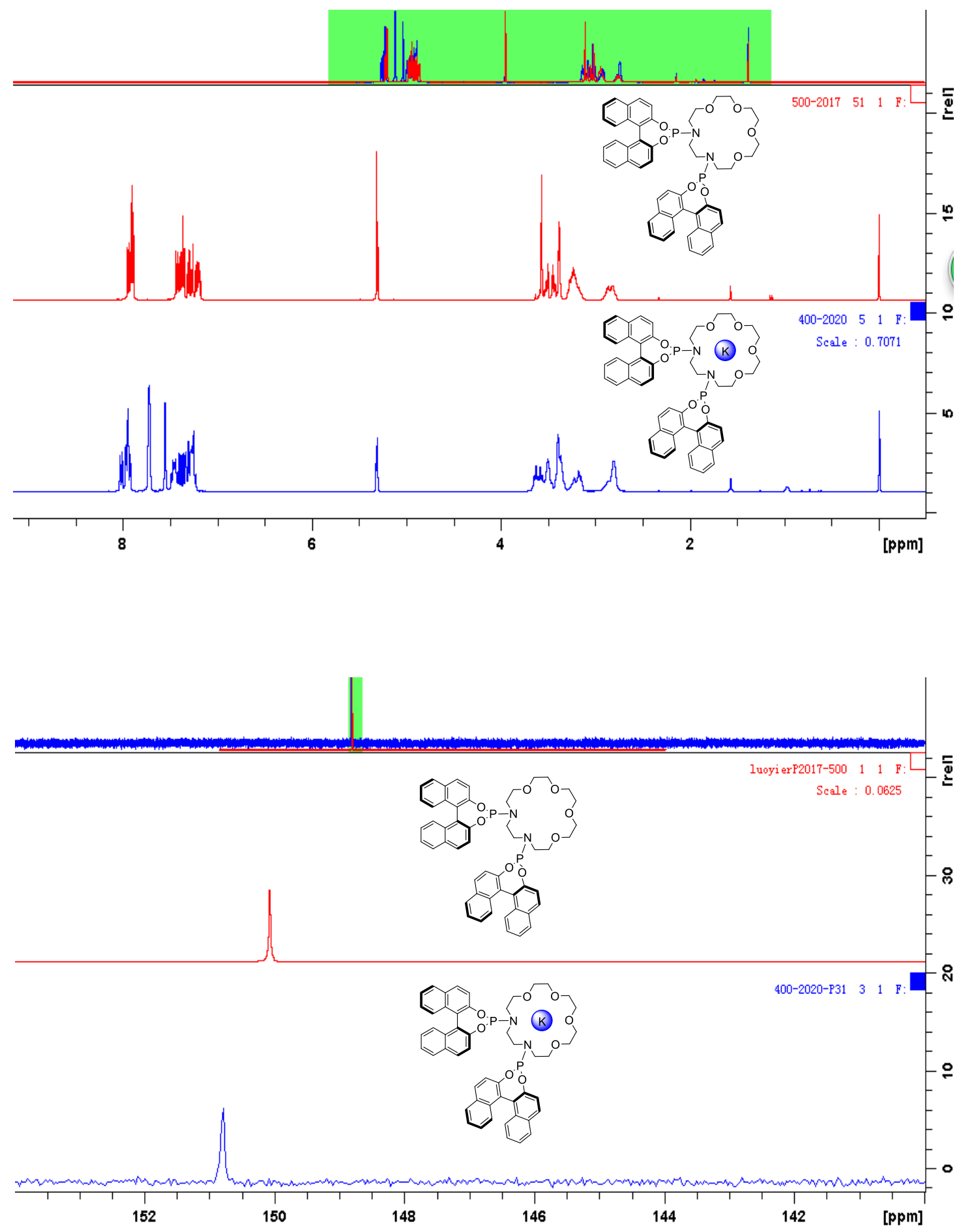


\section{(2) Determination of $\mathbf{L 3}-\mathrm{K}^{+}$association constant by Scatchard plot}

A general procedure for ${ }^{1} \mathrm{H}$ NMR titration is as follows: the required amount of $\mathrm{KBArF}$ and $(S, S)-\mathbf{L 3}$ ligand $(0.005 \mathrm{mmol})$ was dissolved in $0.5 \mathrm{~mL}$ mixed solvent $\left(\mathrm{d}_{8}\right.$-Toluene $\left./ \mathrm{d}_{8}-\mathrm{THF}=7: 3\right)$ in an NMR tube. This procedure ensures a constant concentration of the Host molecule $((S, S)-\mathbf{L 3})$ throughout the NMR titration. The data used for the calculation of association constant $\mathrm{K}_{\mathrm{a}}$ are as follows:

\begin{tabular}{|c|c|c|c|c|}
\hline $\mathrm{M}_{\mathrm{G}}(\mathrm{mmol})$ & {$[\mathrm{G}](\mathrm{mol} / \mathrm{L})$} & $\delta(\mathrm{ppm})$ & $\Delta \delta(\mathrm{ppm})$ & $\Delta \delta /[\mathrm{G}](\mathrm{ppm} * \mathrm{~L} / \mathrm{mol})$ \\
\hline 0 & 0 & 3.3721 & 0 & --- \\
\hline 0.001 & 0.002 & 3.3655 & 0.0066 & 3.3000 \\
\hline 0.002 & 0.004 & 3.3597 & 0.0124 & 3.1000 \\
\hline 0.003 & 0.006 & 3.3544 & 0.0177 & 2.9500 \\
\hline 0.004 & 0.008 & 3.3496 & 0.0225 & 2.8125 \\
\hline 0.005 & 0.01 & 3.3455 & 0.0266 & 2.6600 \\
\hline 0.006 & 0.012 & 3.3416 & 0.0305 & 2.5417 \\
\hline 0.007 & 0.014 & 3.3382 & 0.0339 & 2.4214 \\
\hline 0.008 & 0.016 & 3.3351 & 0.0370 & 2.3125 \\
\hline 0.009 & 0.018 & 3.3322 & 0.0399 & 2.2167 \\
\hline 0.01 & 0.02 & 3.3299 & 0.0422 & 2.1100 \\
\hline
\end{tabular}

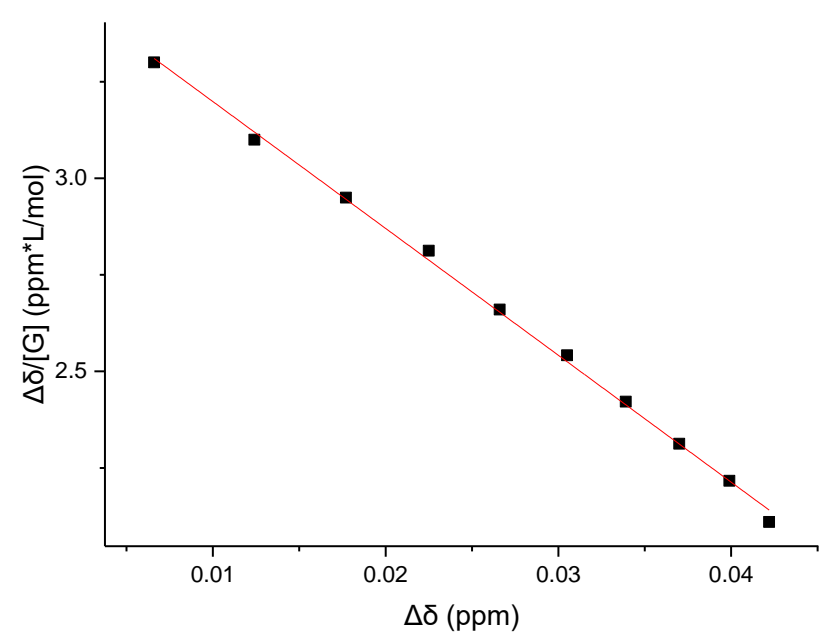

\begin{tabular}{|c|c|c|c|c|}
\hline \multicolumn{2}{|c|}{ Intercept } & \multicolumn{2}{c|}{ Slope } & Statistics \\
\hline Value & Standard Error & Value & Standard Error & R-Square \\
\hline 3.52711 & 0.01422 & -32.8492 & 0.4864 & 0.99803 \\
\hline
\end{tabular}

Linear data fitting using Scatchard plot $\left(\frac{\Delta}{[\mathrm{G}]}=-\Delta \mathrm{K}+\triangle_{0} \mathrm{~K}\right)$ delivered the association constant $\mathrm{K}_{\mathrm{a}}=32.85 \pm 0.49$. 


\section{(3) Determination of $\mathbf{L 3}-\mathrm{K}^{+}$molar ratio by Job Plot analysis}

To further understand the complexation between $(S, S)-\mathbf{L} \mathbf{3}$ and $\mathrm{KBArF}$, we used the classical Job Plot analysis to measure the Host-Guest ratio. A general procedure for ${ }^{1} \mathrm{H}$ NMR titration is as follows: the required amount of $\mathrm{KBArF}$ and $(S, S)-\mathbf{L} 3$ ligand was dissolved in $0.5 \mathrm{~mL}$ mixed solvent $\left(\mathrm{d}_{8}\right.$-Toluene $\left./ \mathrm{d}_{8}-\mathrm{THF}=7: 3\right)$ in an NMR tube. This procedure ensures a constant concentration of a total amount of the $(S, S)-\mathbf{L 3}$ and $\operatorname{KBArF}([$ Host $]+[$ Guest $]=0.02 \mathrm{~mol} / \mathrm{L})$.

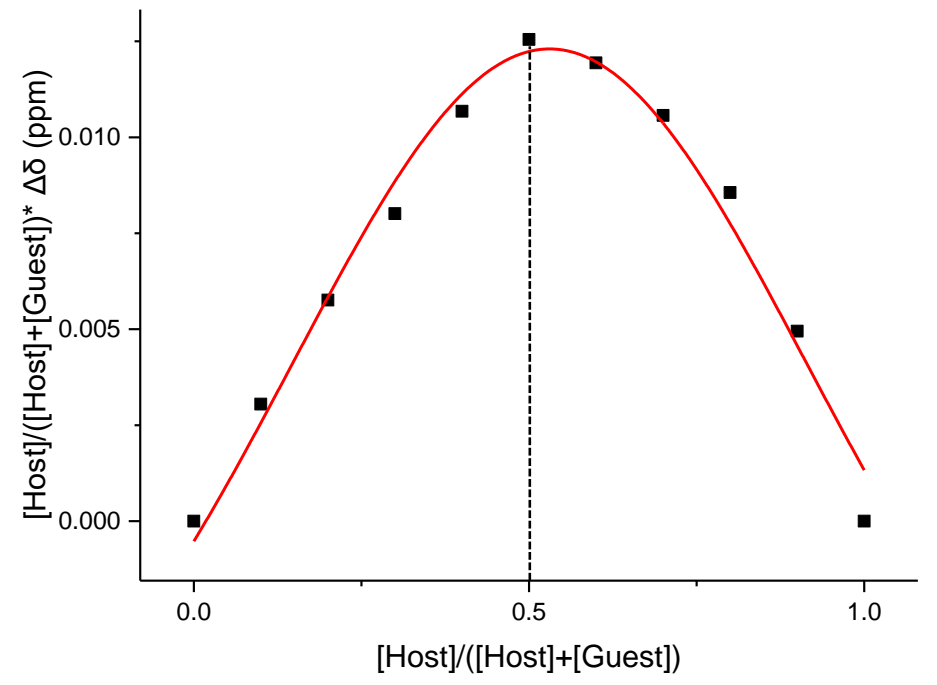

\begin{tabular}{|c|c|c|c|c|}
\hline entry & $[$ Host $] /[$ Host $]+[$ Guest $])$ & $\delta(\mathrm{ppm})$ & $\Delta \delta(\mathrm{ppm})$ & $[$ Host $] /[$ Host $]+[$ Guest $]) * \Delta \delta(\mathrm{ppm})$ \\
\hline 1 & 0 & --- & --- & 0 \\
\hline 2 & 0.1 & 3.3600 & 0.0305 & 0.00305 \\
\hline 3 & 0.2 & 3.3617 & 0.0288 & 0.00576 \\
\hline 4 & 0.3 & 3.3638 & 0.0267 & 0.00801 \\
\hline 5 & 0.4 & 3.3638 & 0.0267 & 0.01068 \\
\hline 6 & 0.5 & 3.3654 & 0.0251 & 0.01255 \\
\hline 7 & 0.6 & 3.3706 & 0.0199 & 0.01194 \\
\hline 8 & 0.7 & 3.3754 & 0.0151 & 0.01057 \\
\hline 9 & 0.8 & 3.3798 & 0.0107 & 0.00856 \\
\hline 10 & 0.9 & 3.3850 & 0.0055 & 0.00495 \\
\hline 11 & 1 & 3.3905 & 0 & 0 \\
\hline
\end{tabular}




\section{References}

1. (a) Huang, C. Y. Determination of binding stoichiometry by the continuous variation method: The job plot. Methods Enzymol. 1982, 87, 509-525. (b) Scatchard, G. The attractions of proteins for small molecules and ions. Ann. N. Y. Acad. Sci. 1949, 51, 660-672. 


\section{Copy of NMR spectra}

\section{NMR spectra of crown ether-based diphosphoramidite ligand}

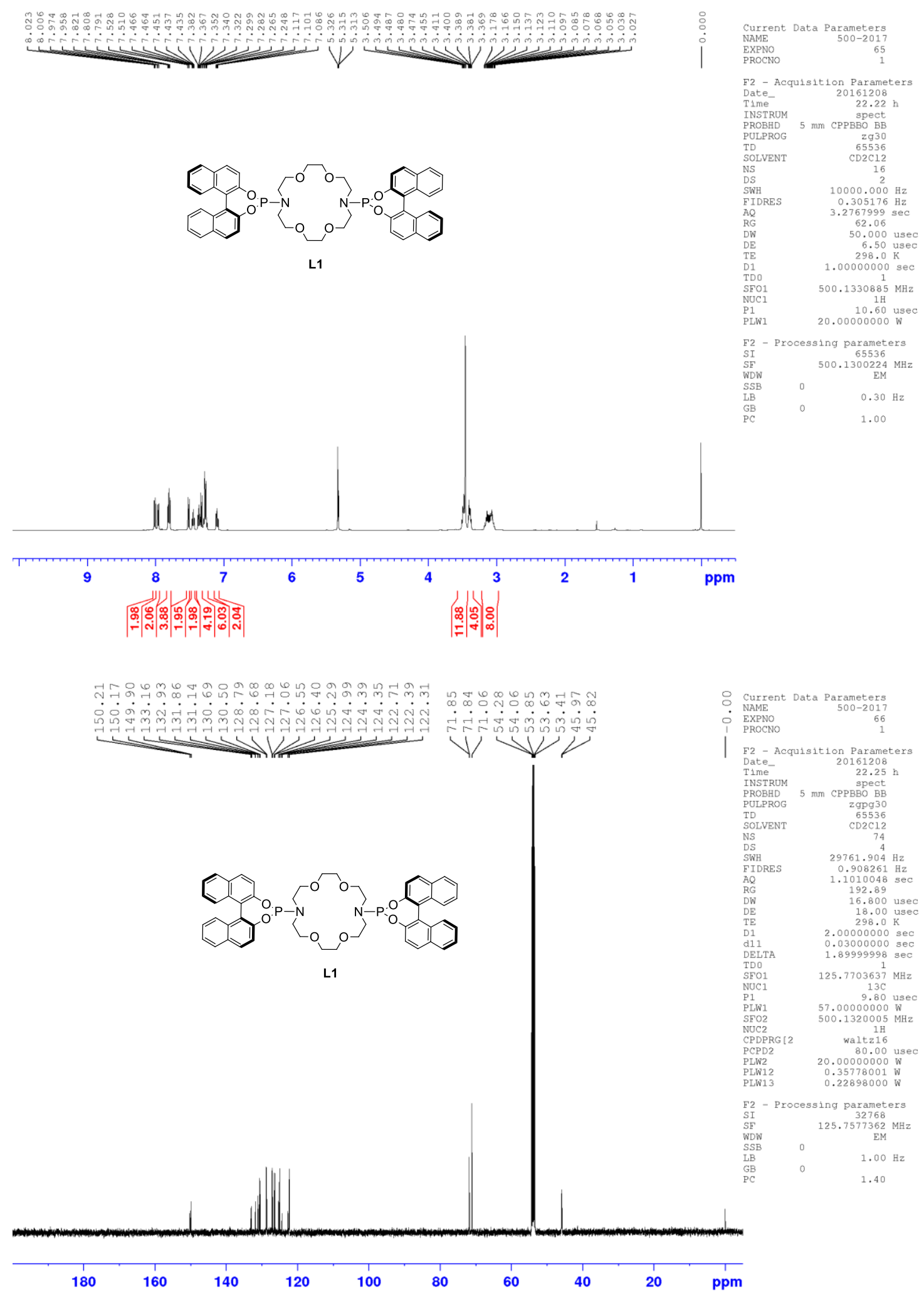



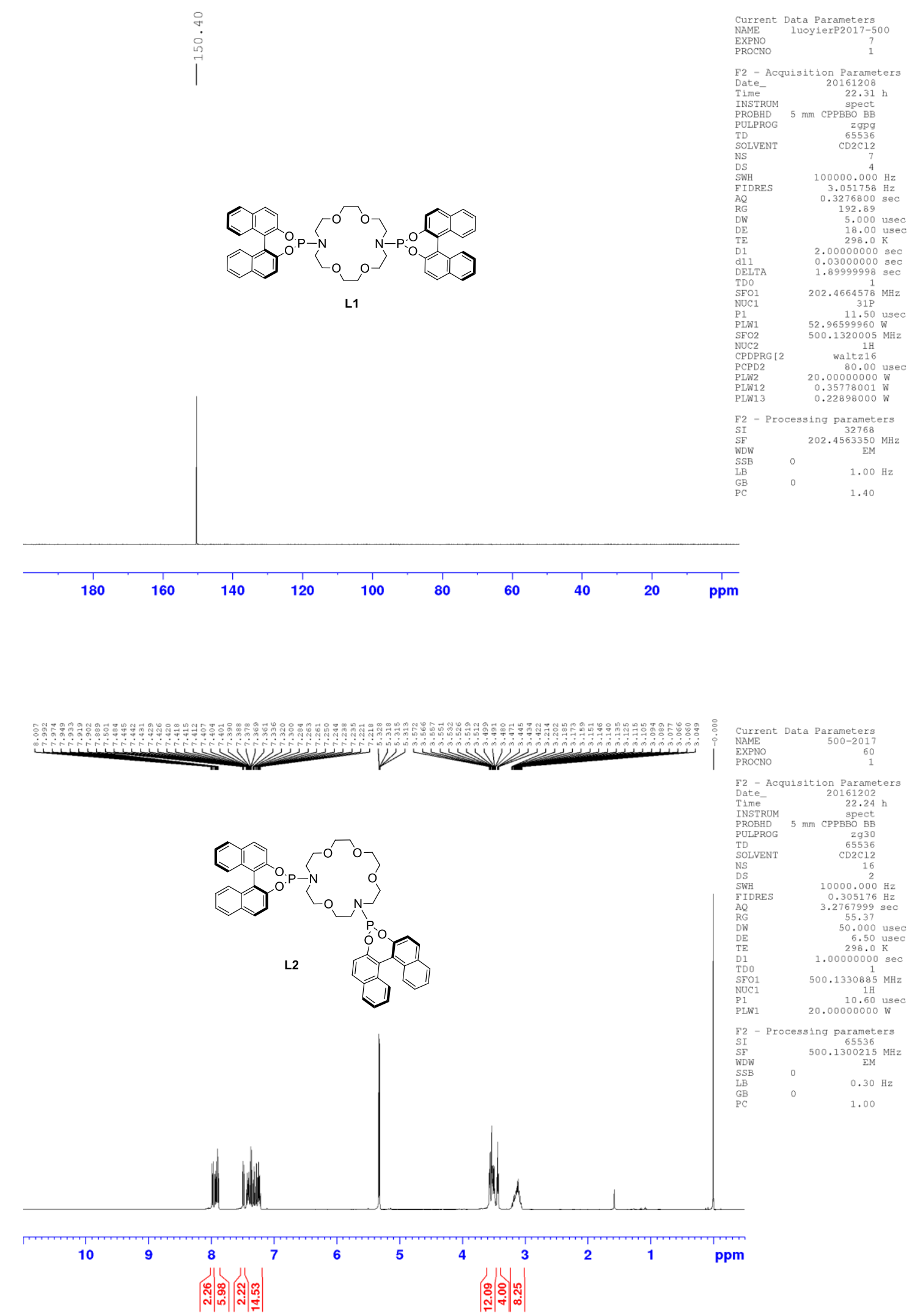

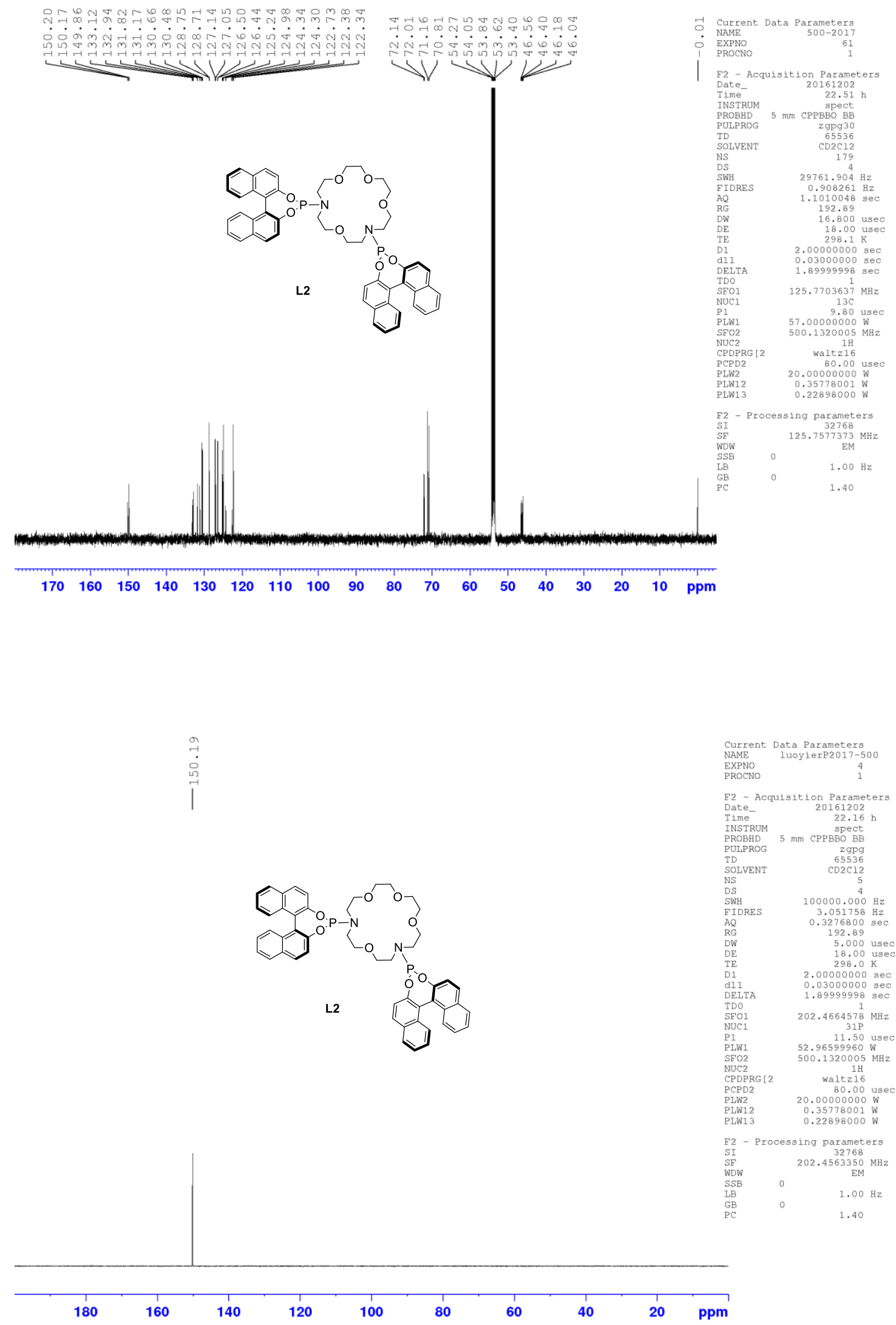
L3
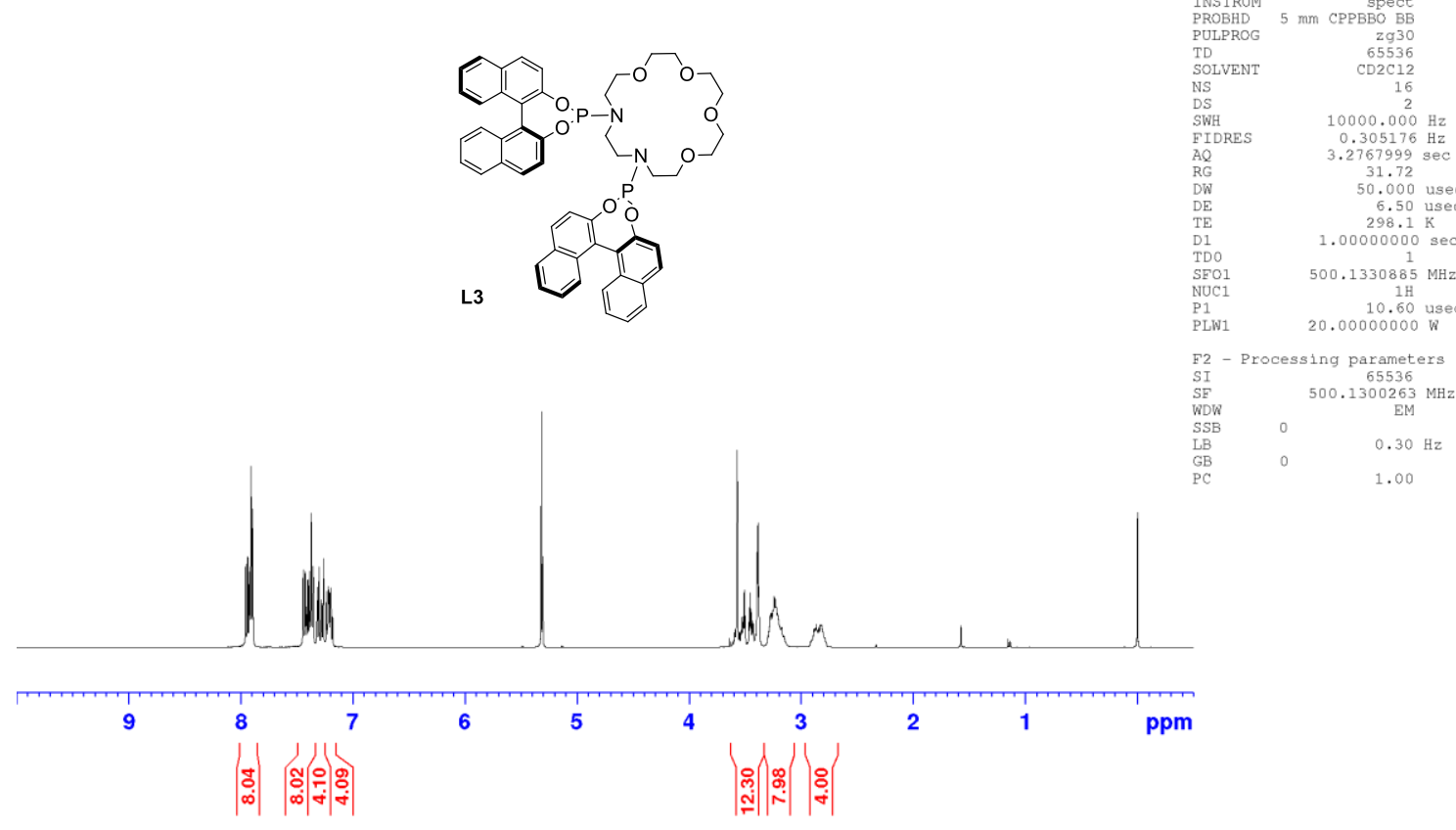

F2 - Processing parameters

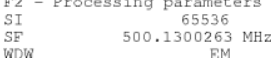

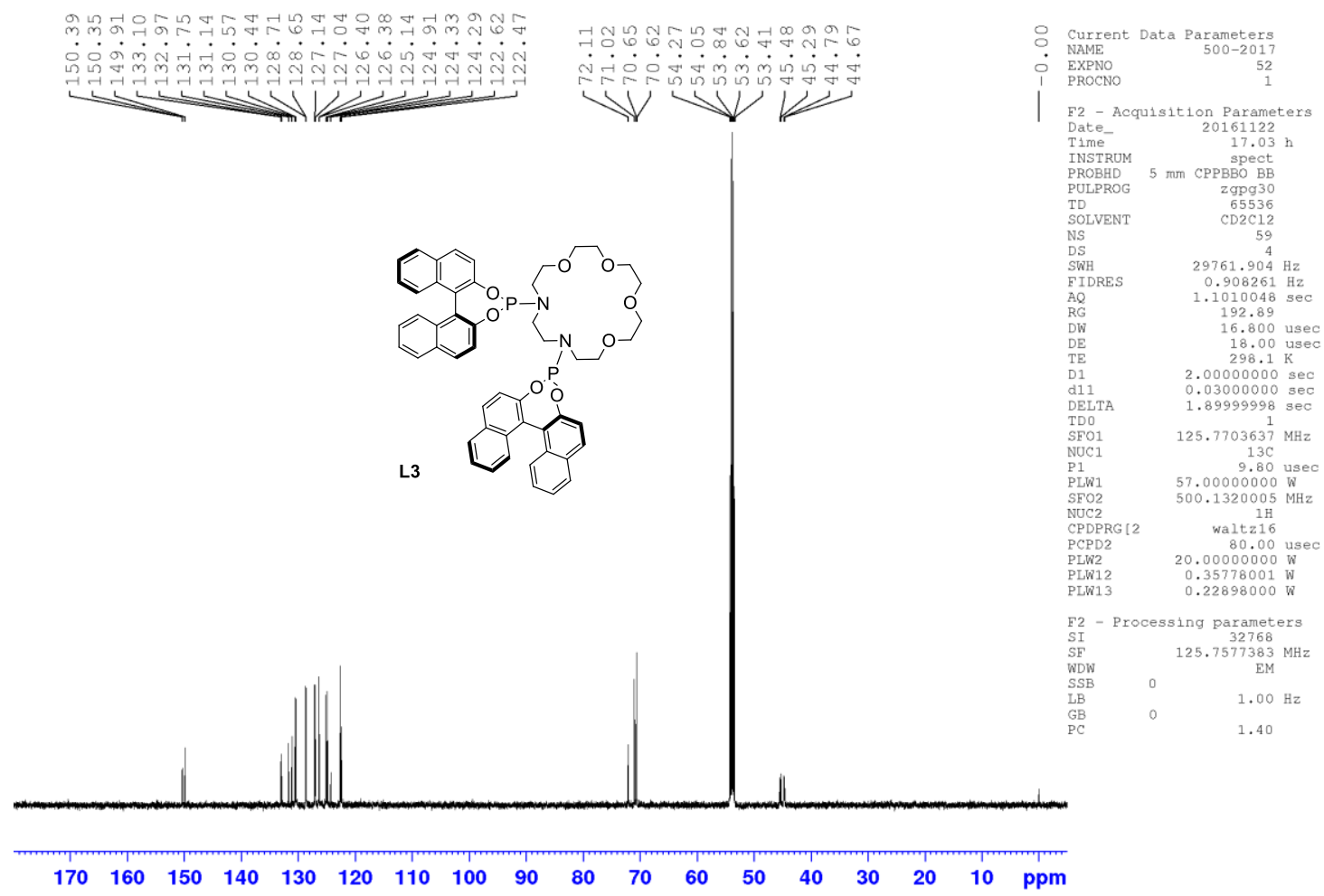



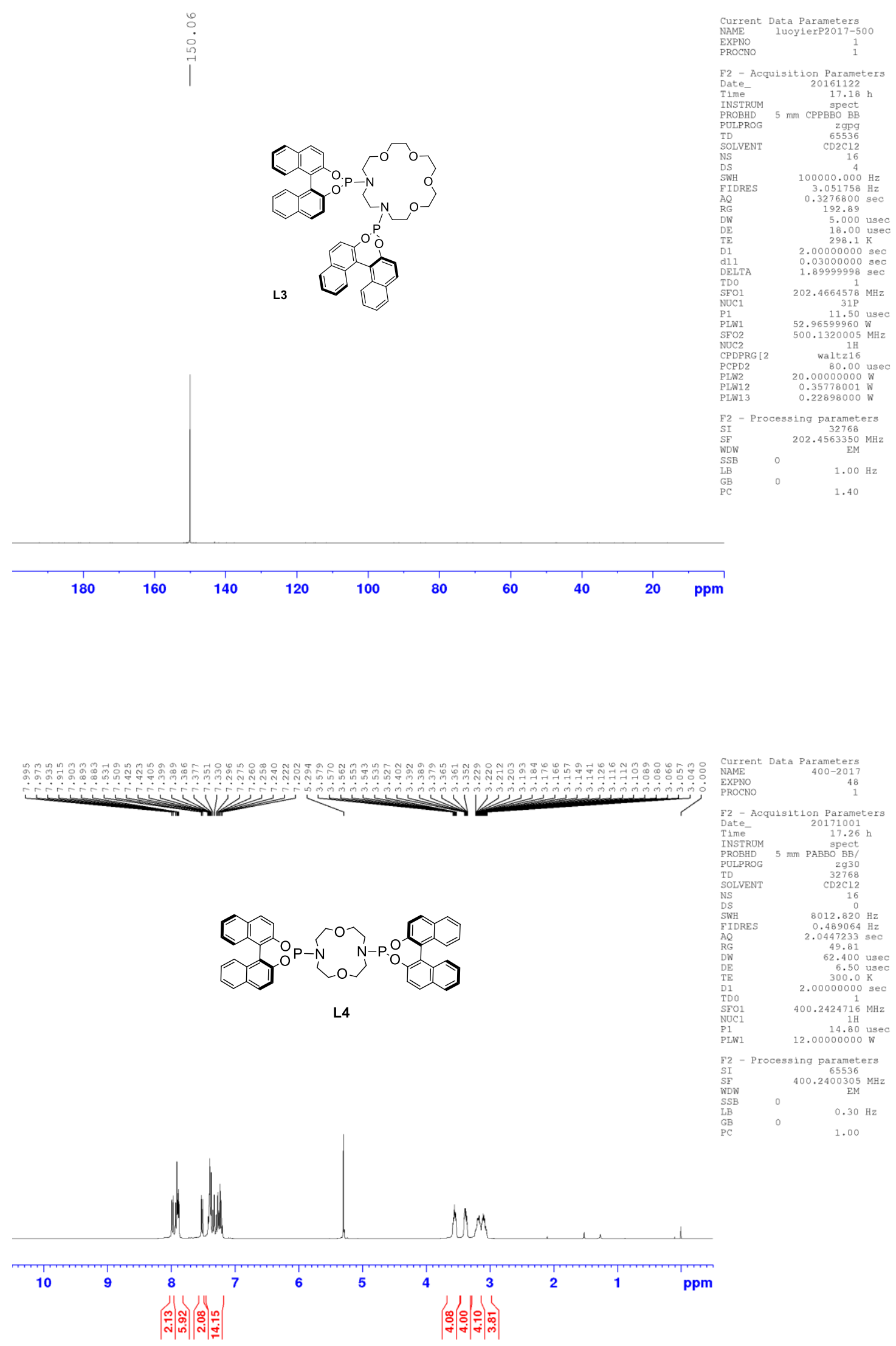

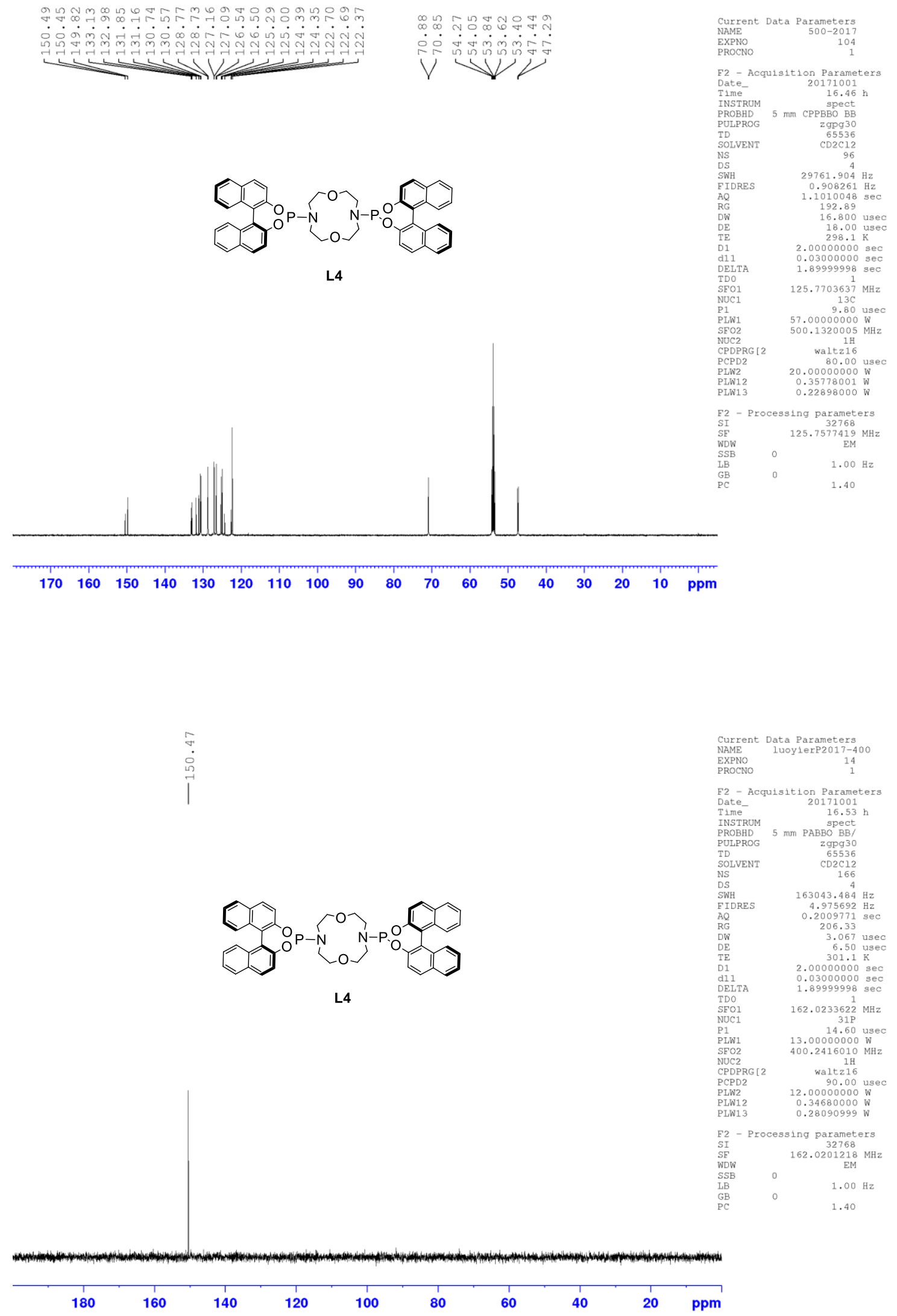


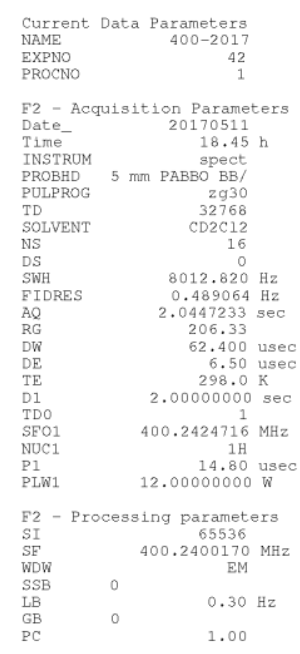

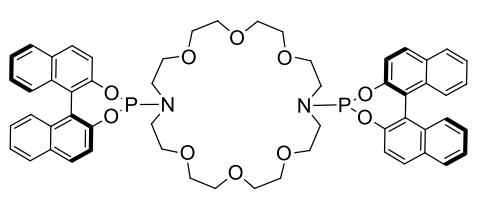

L5

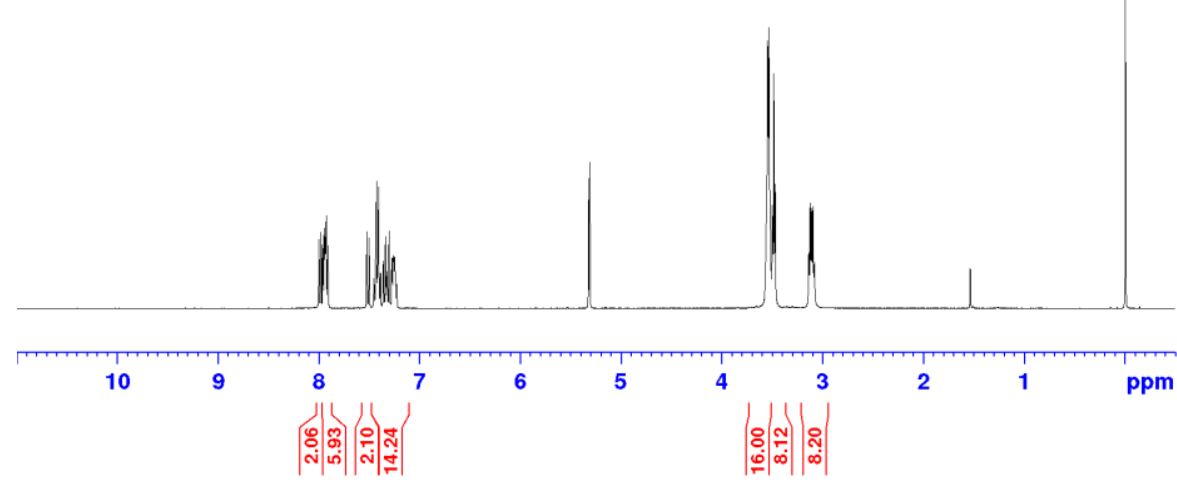

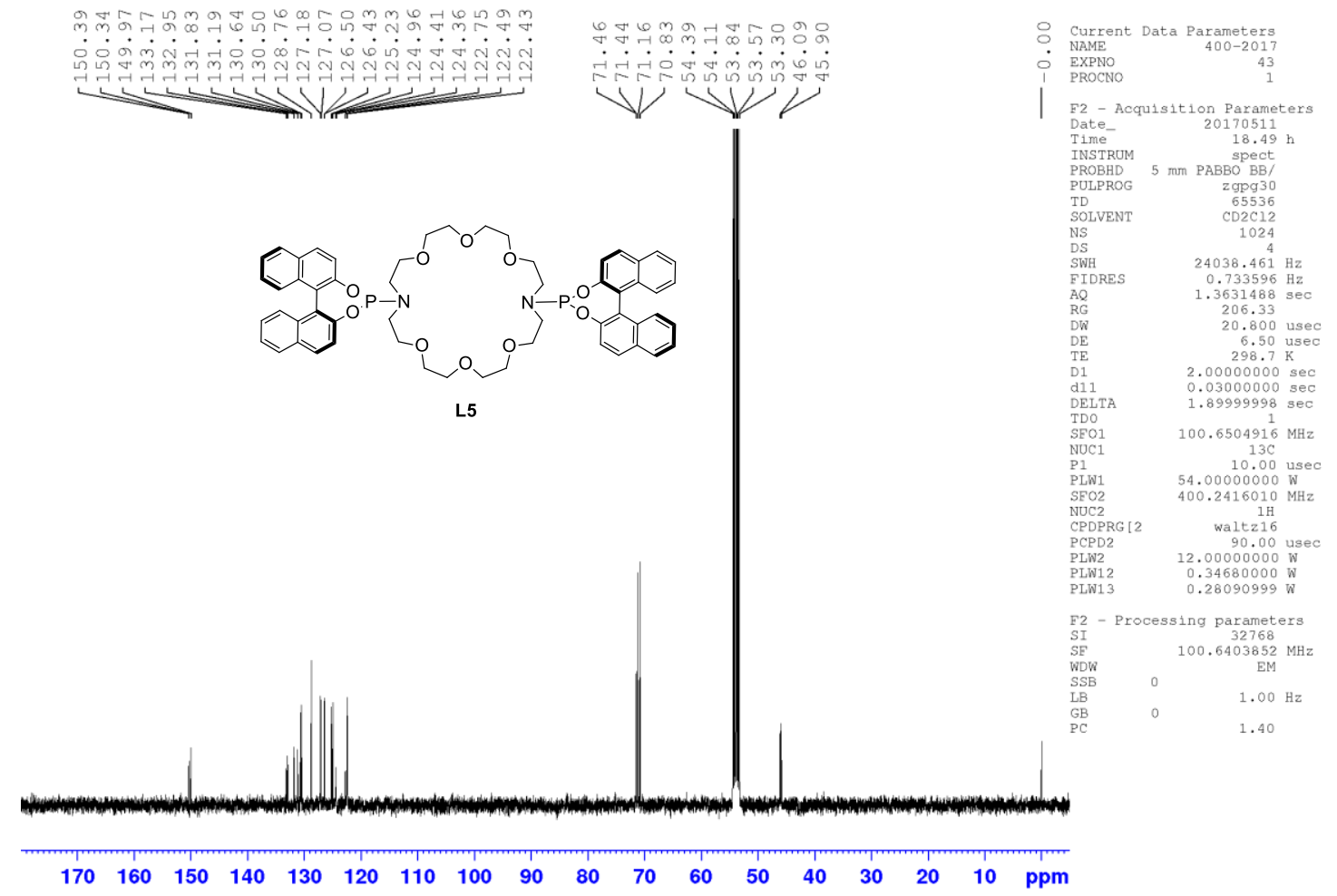




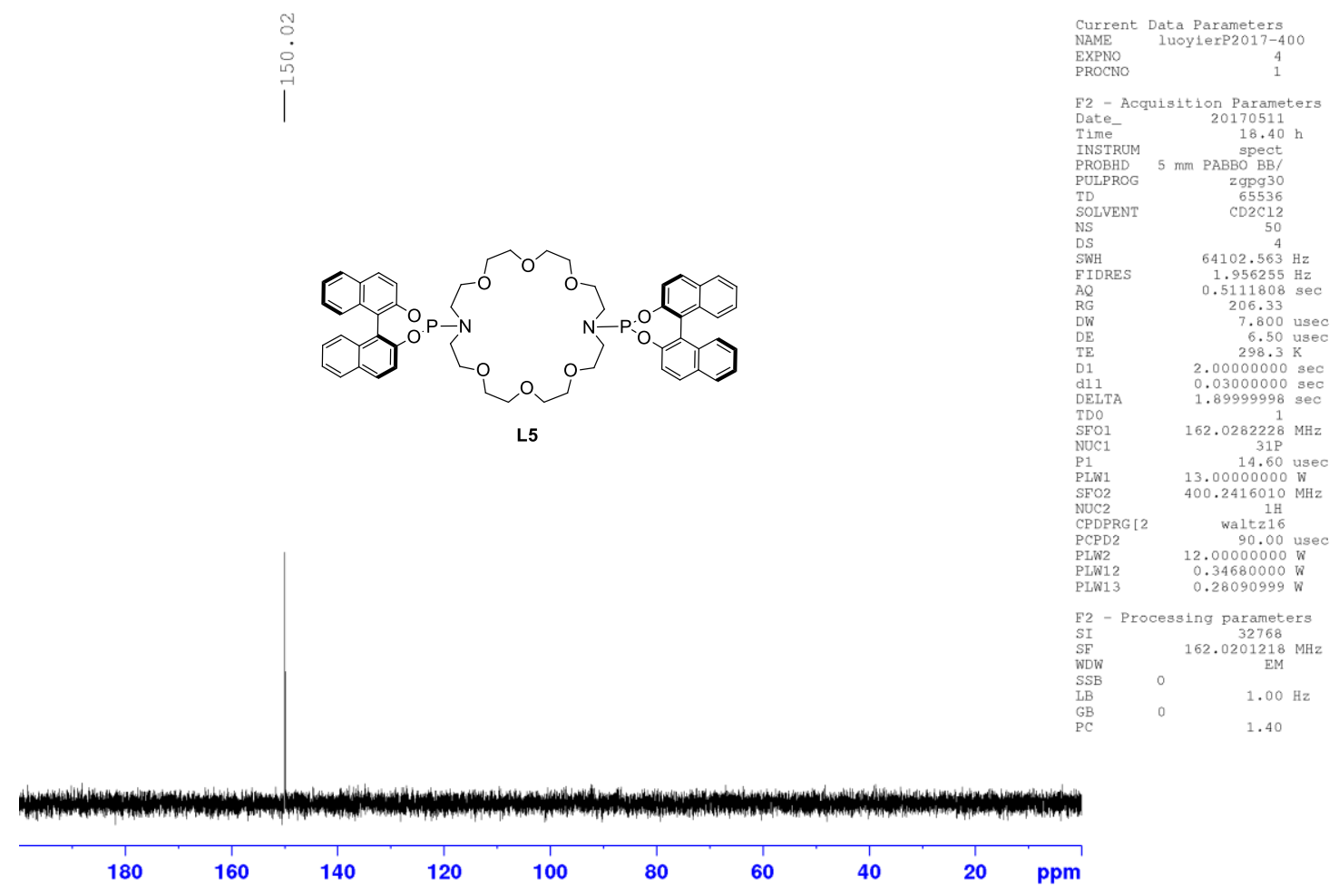


NMR spectra of the amino acid esters products
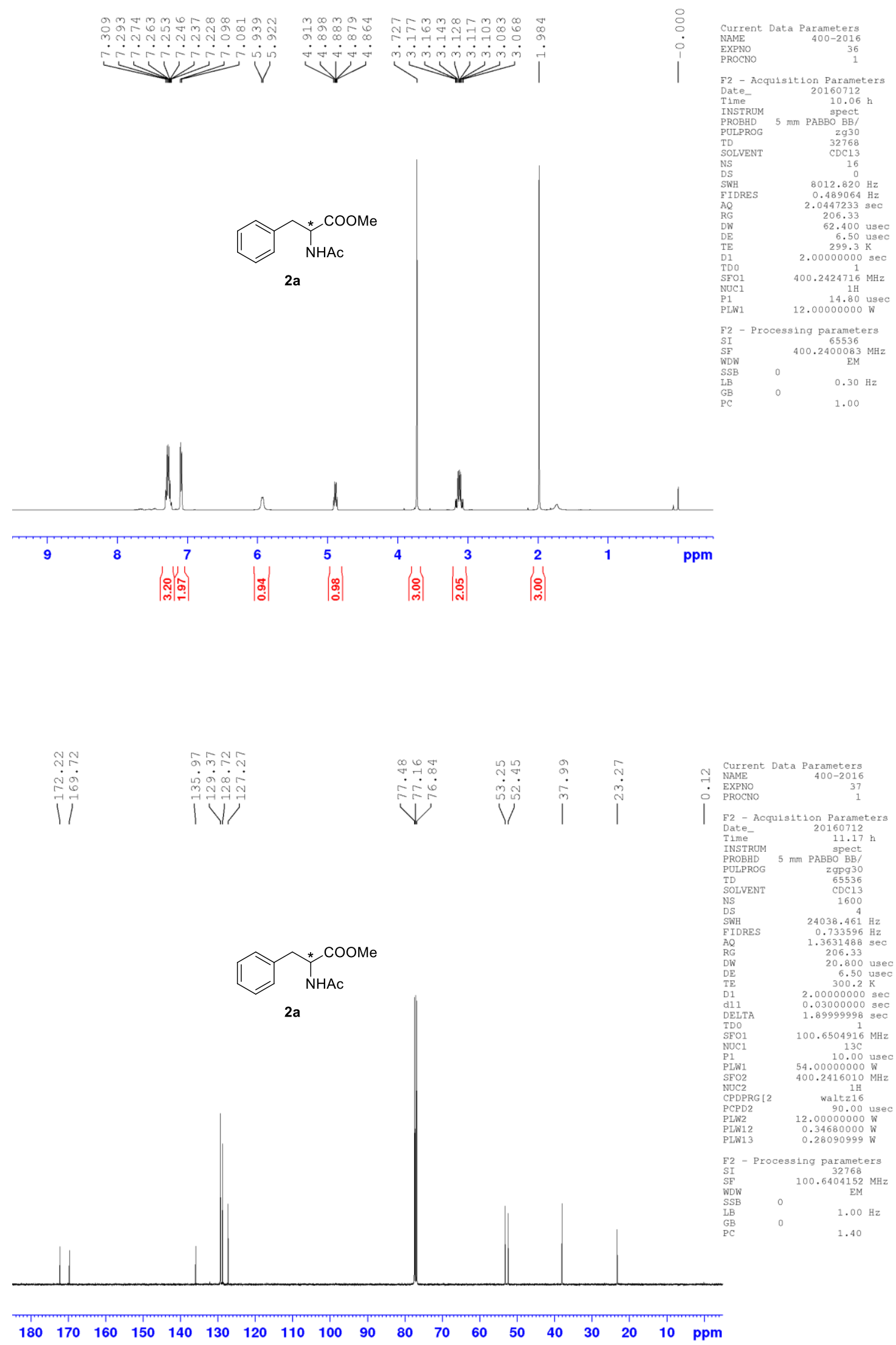

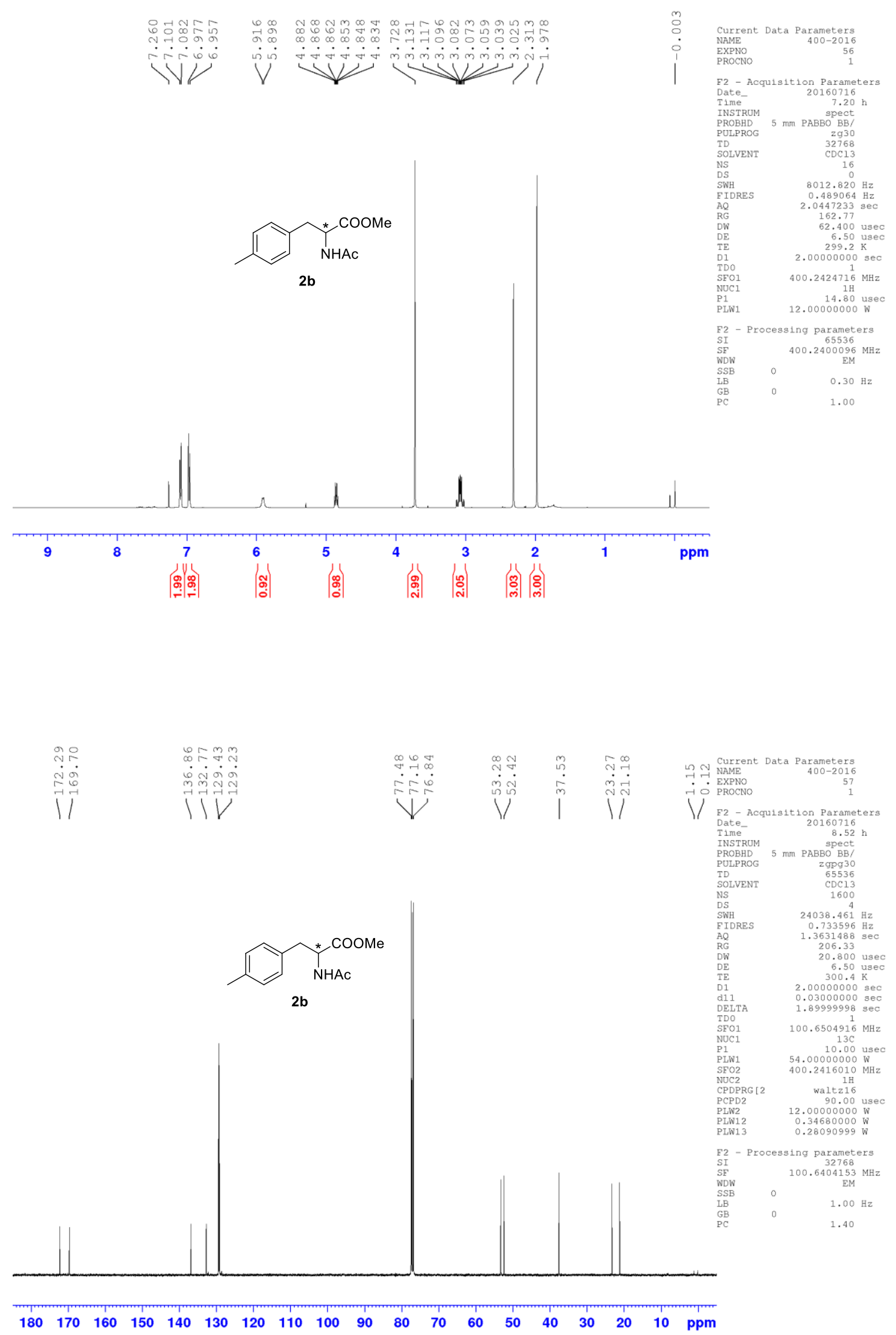

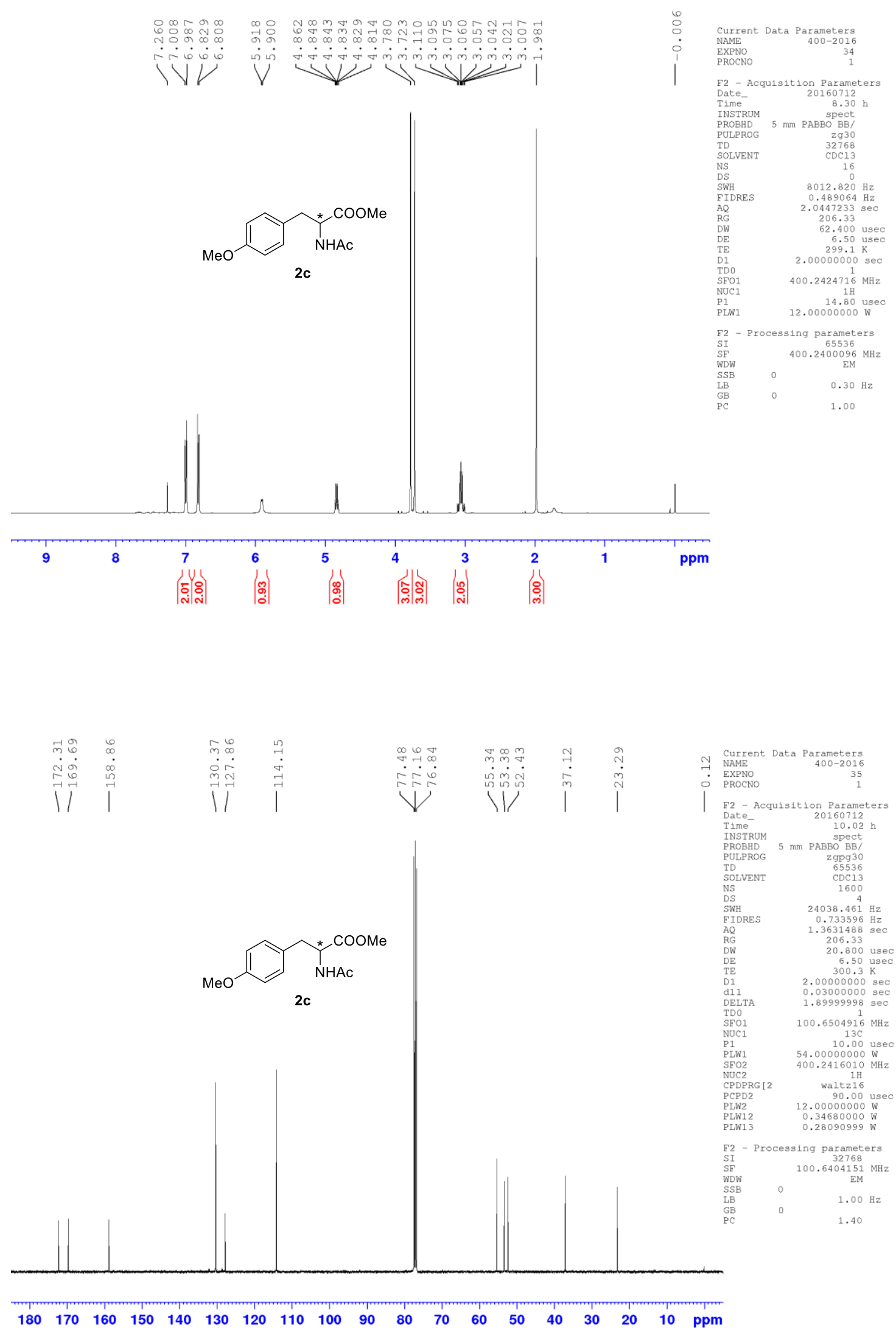

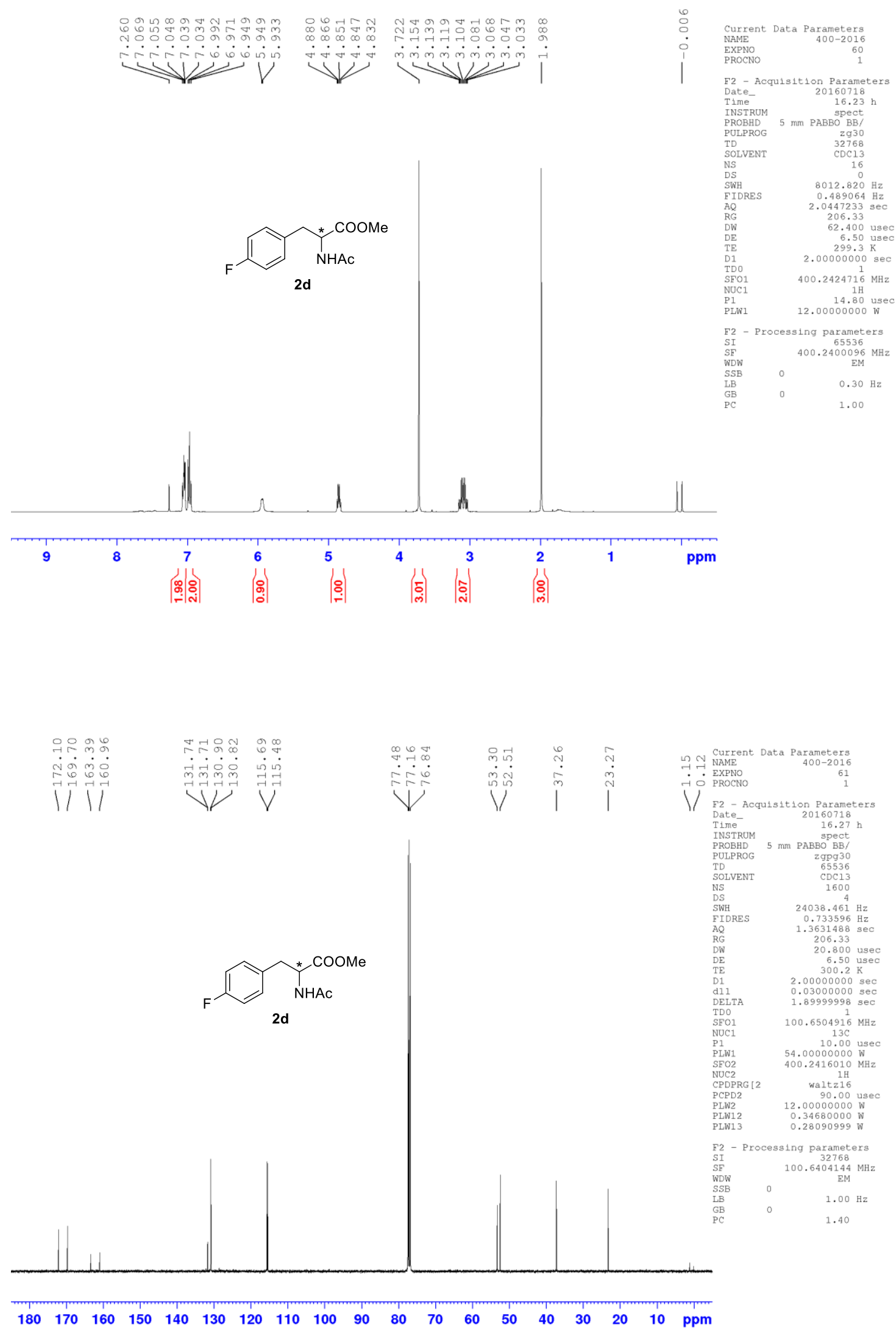

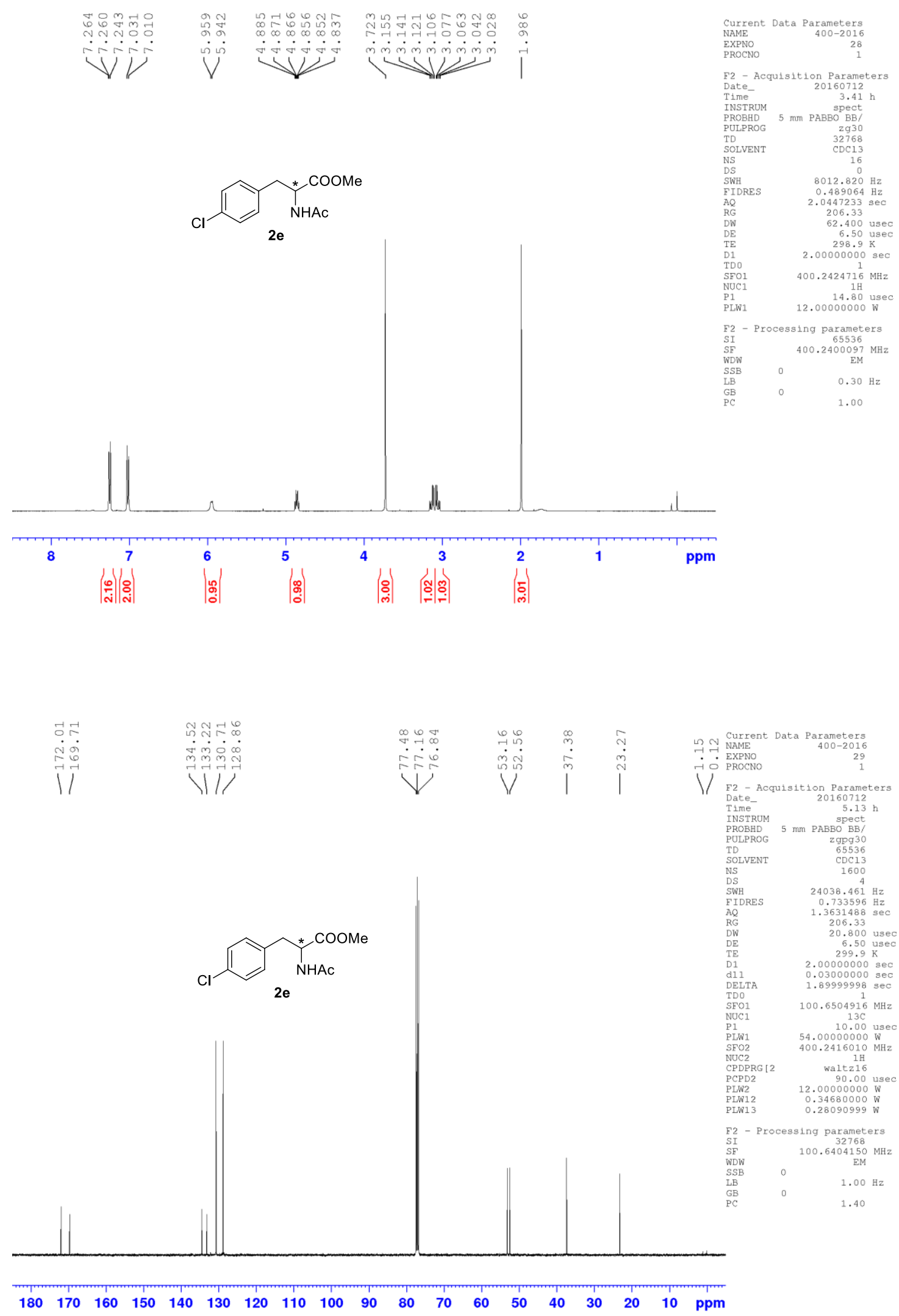

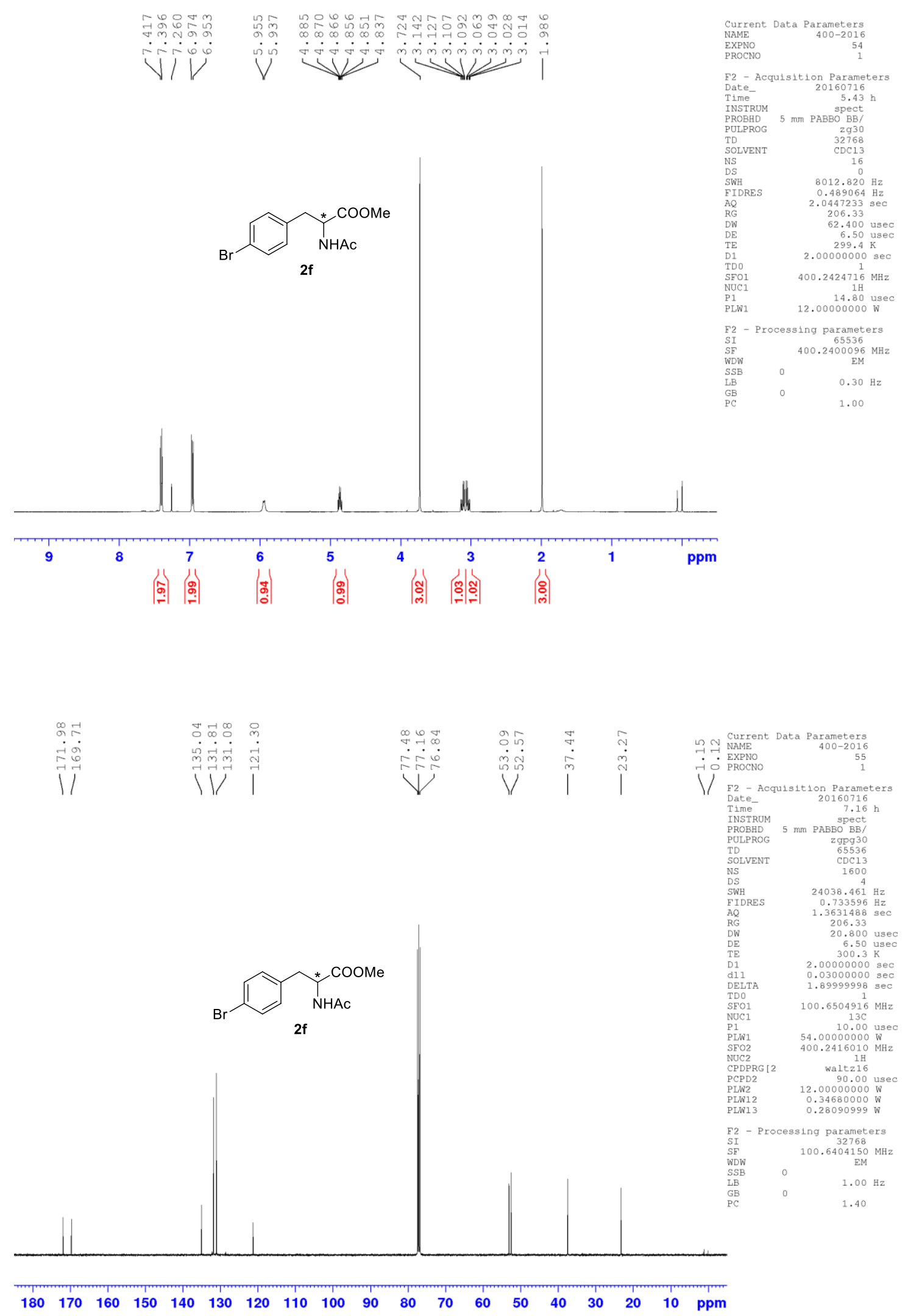

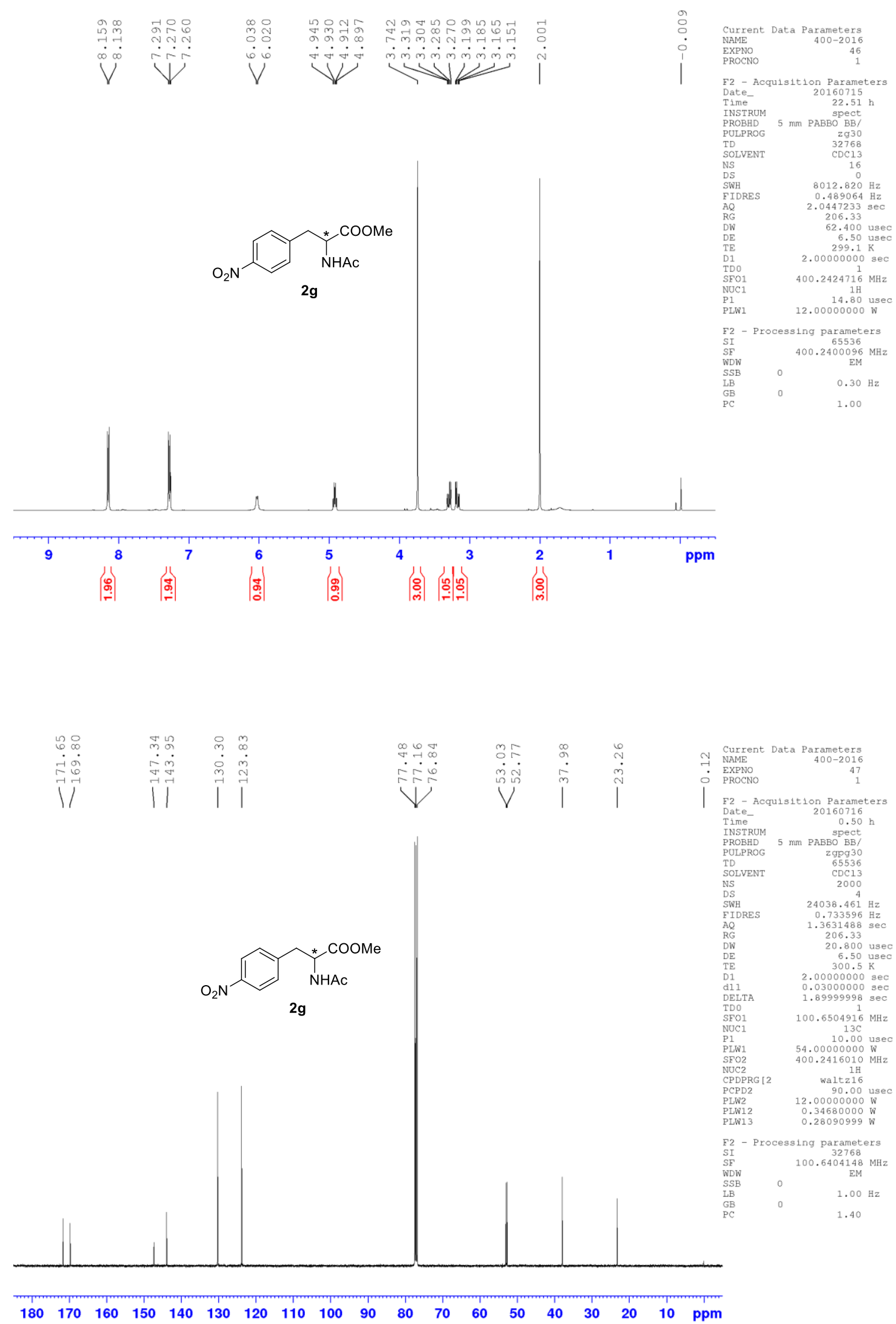

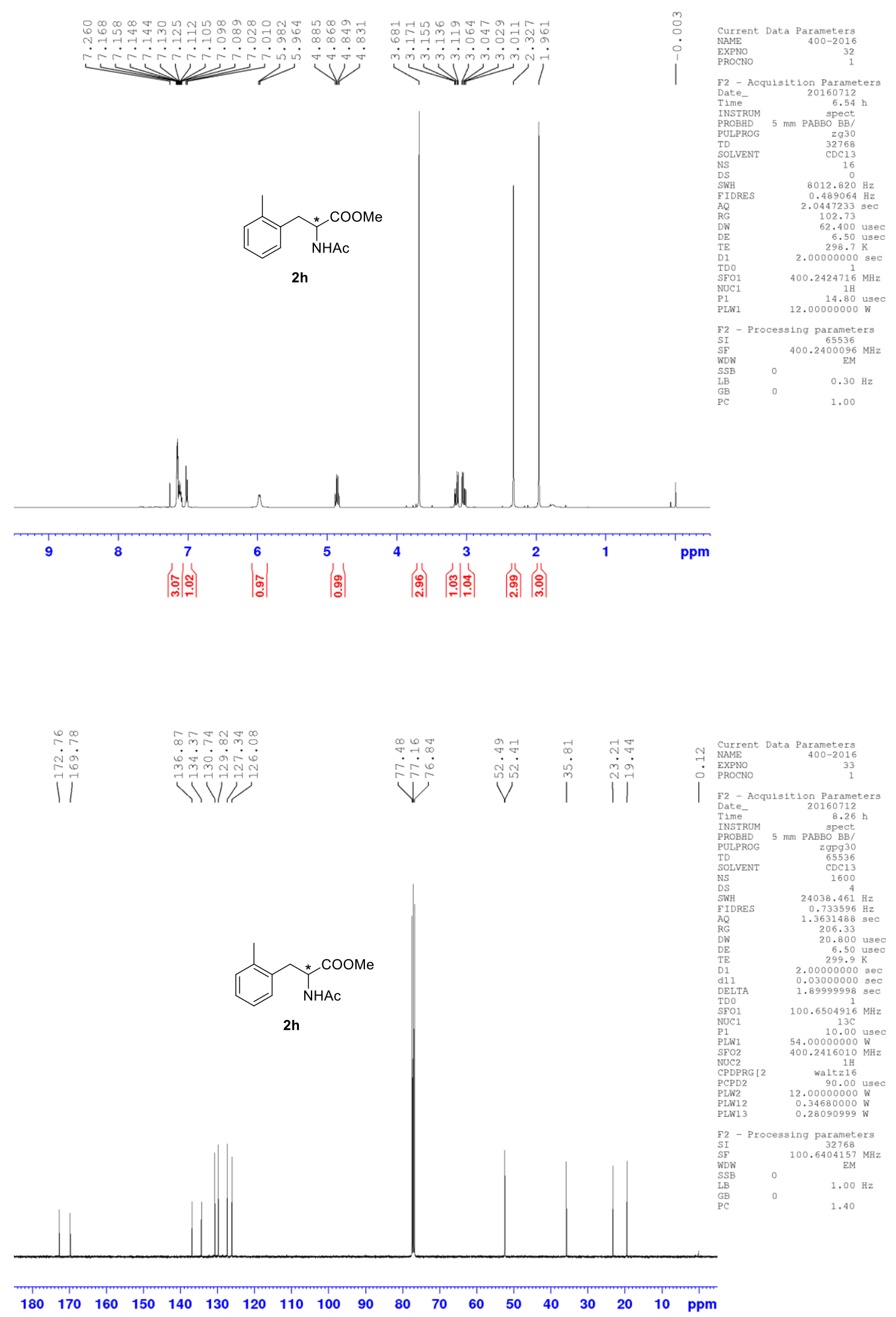

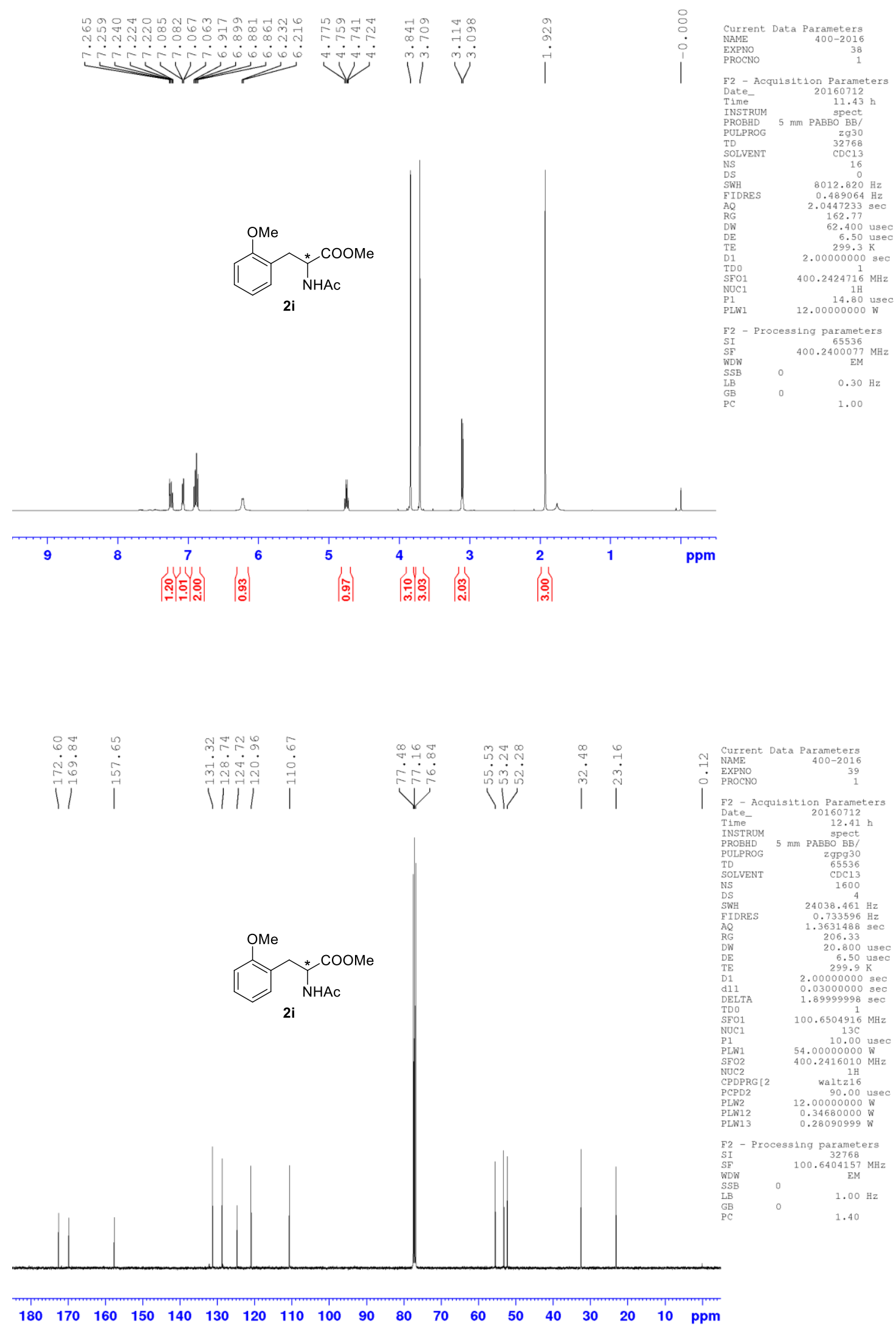

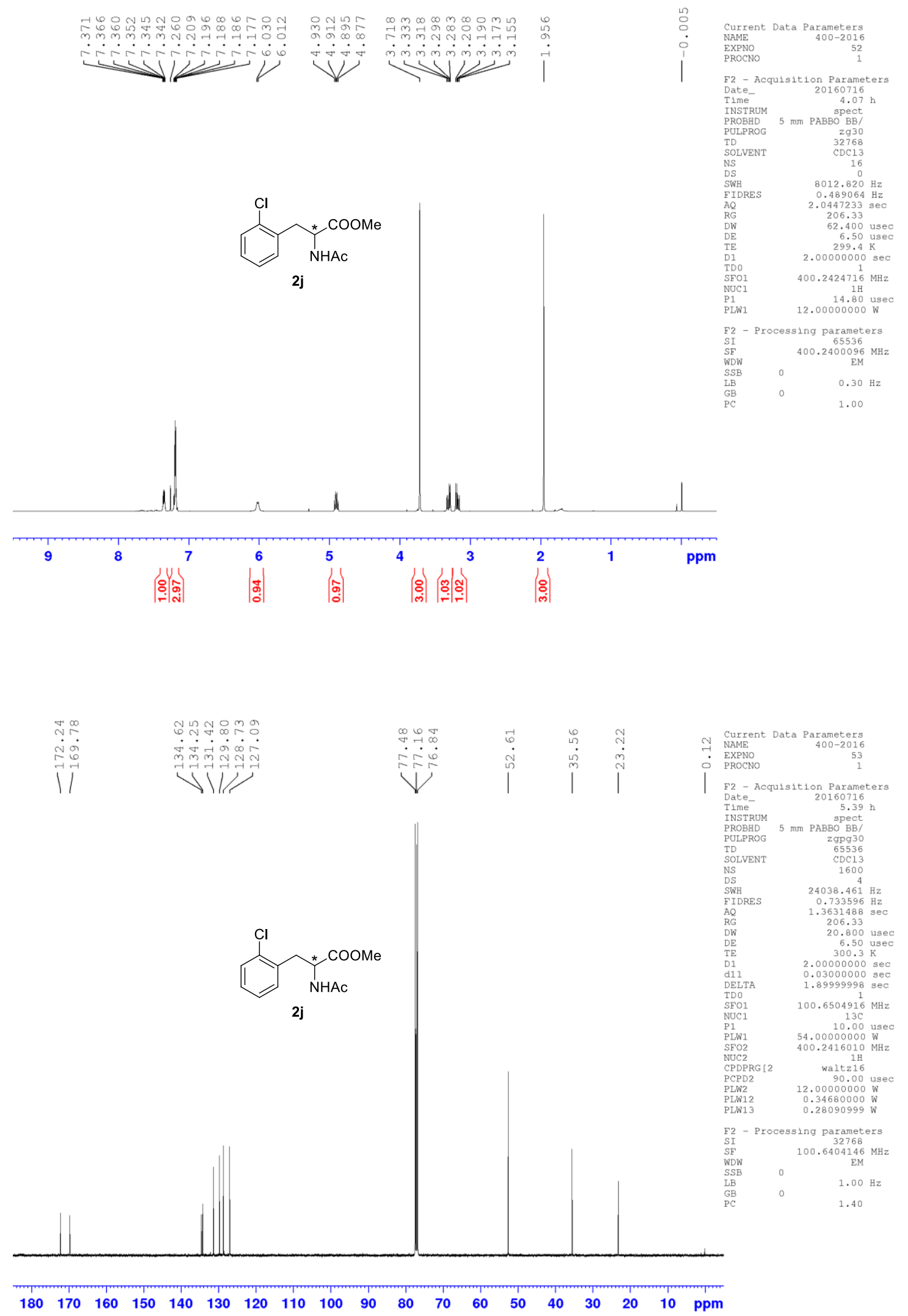

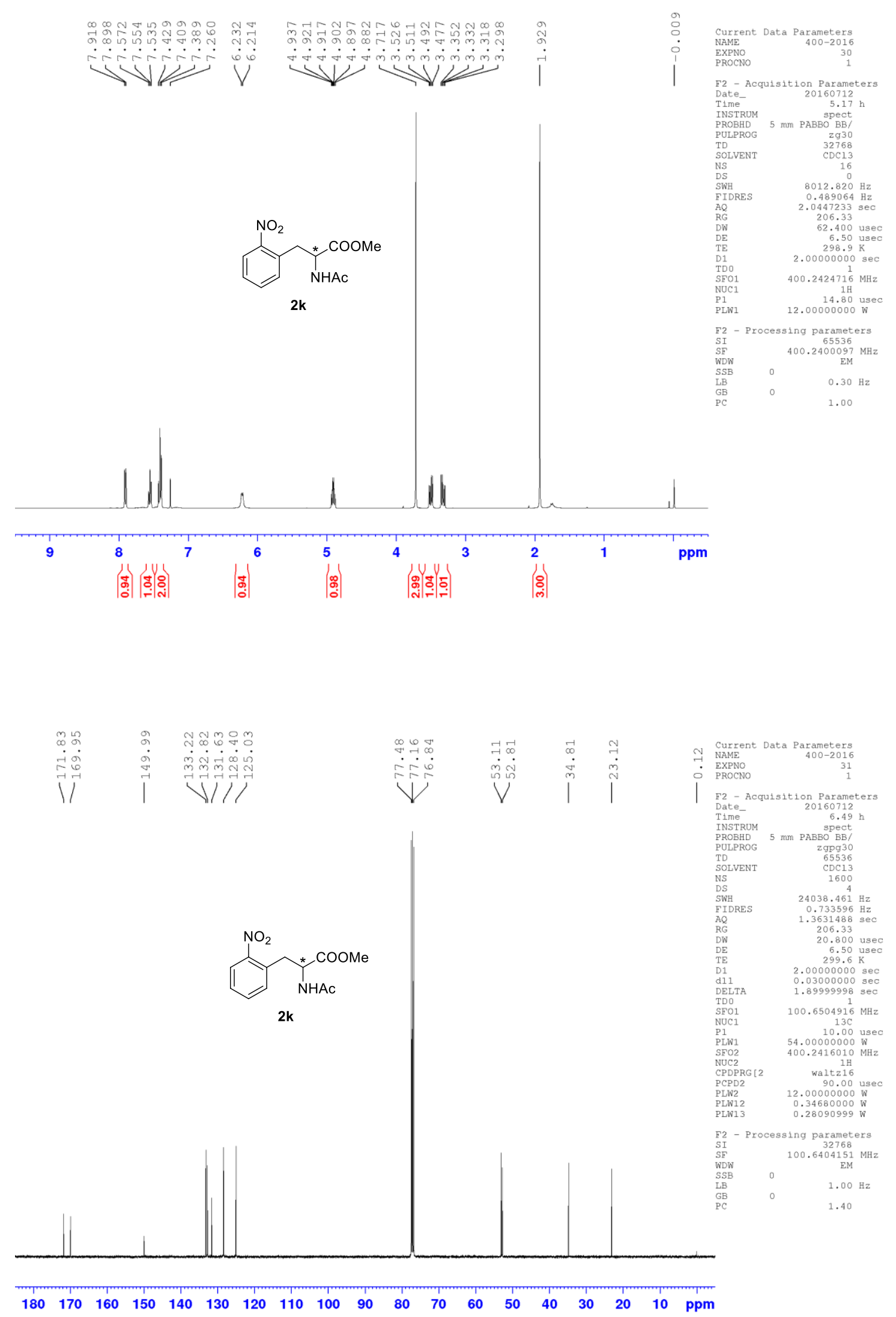

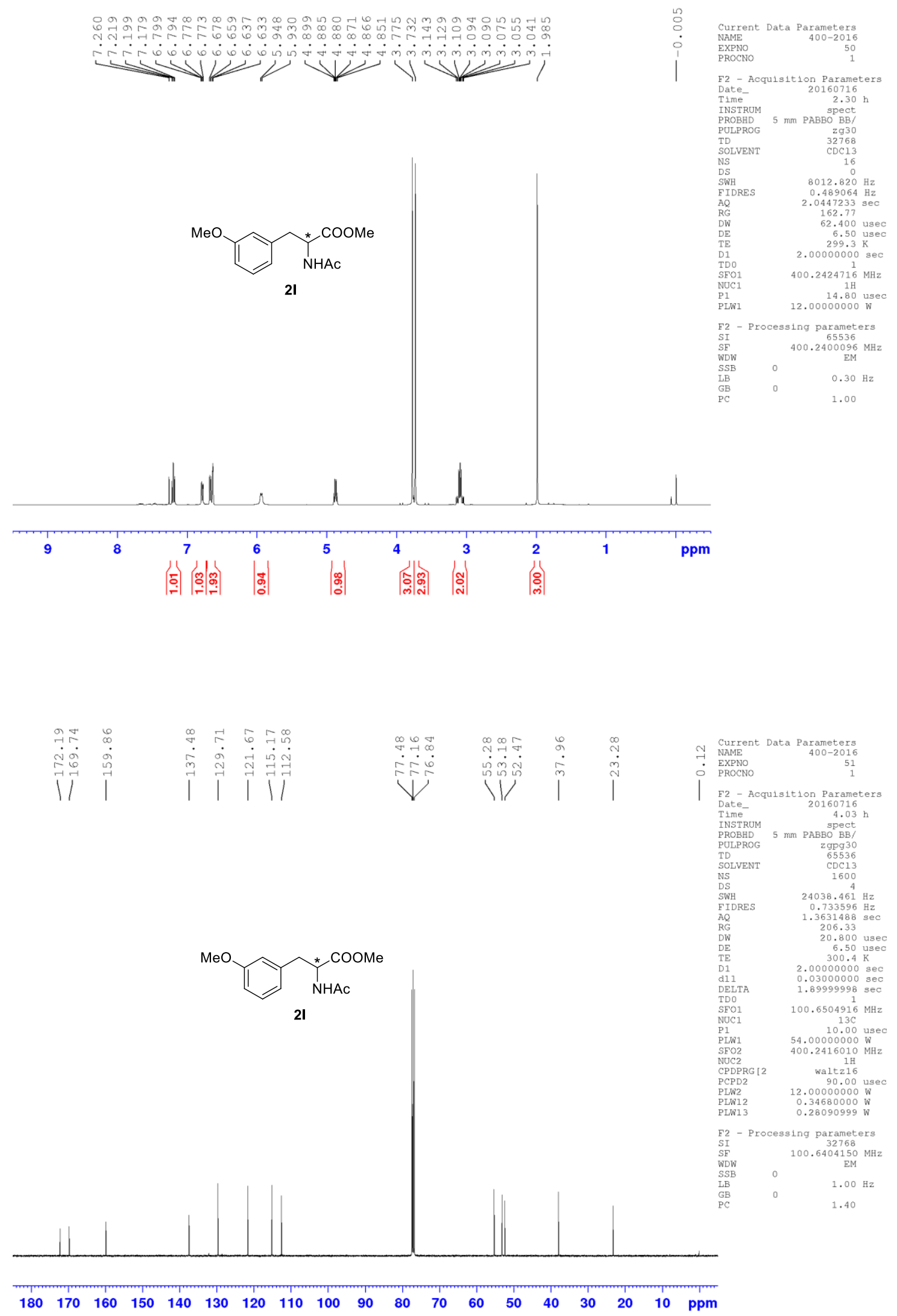

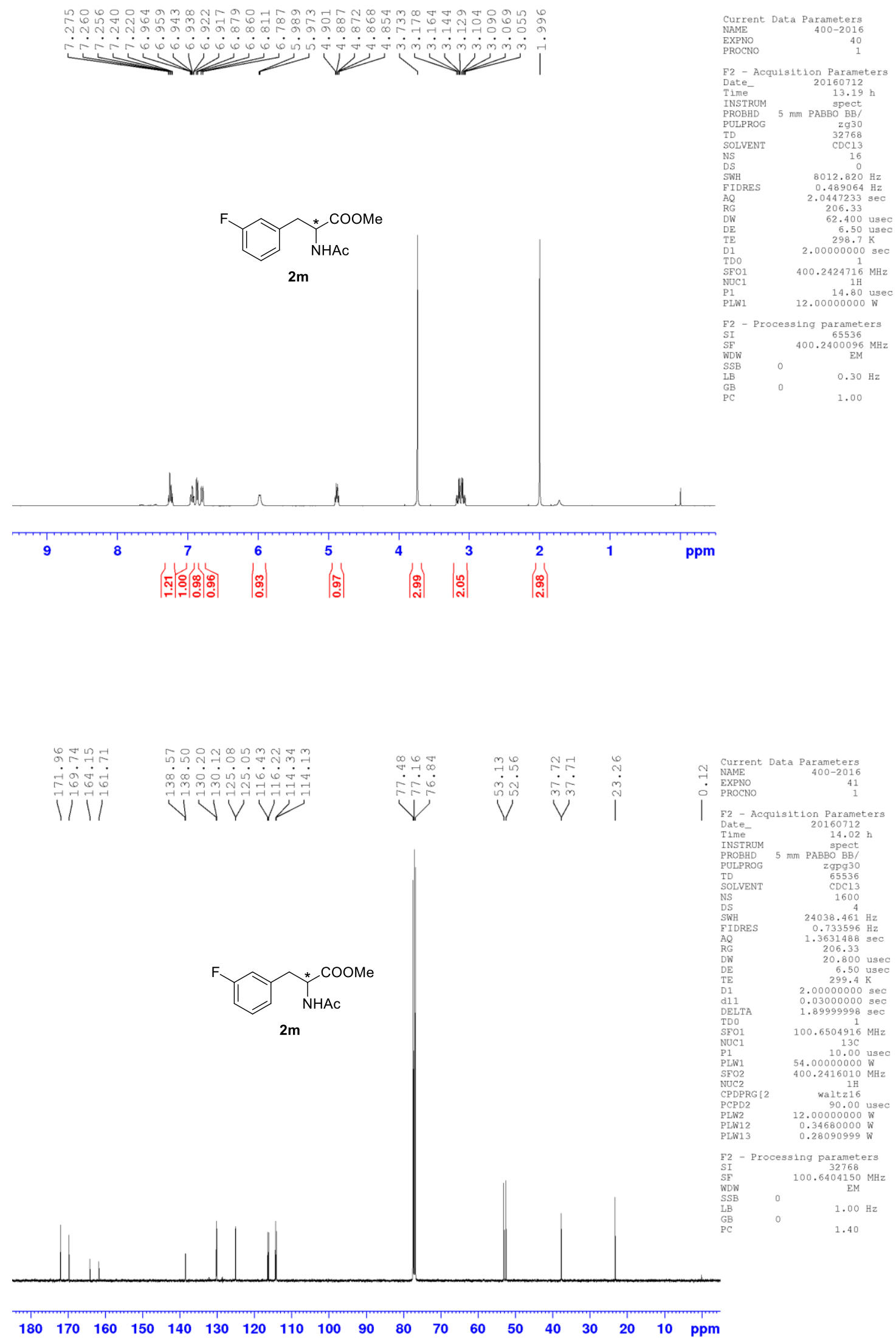

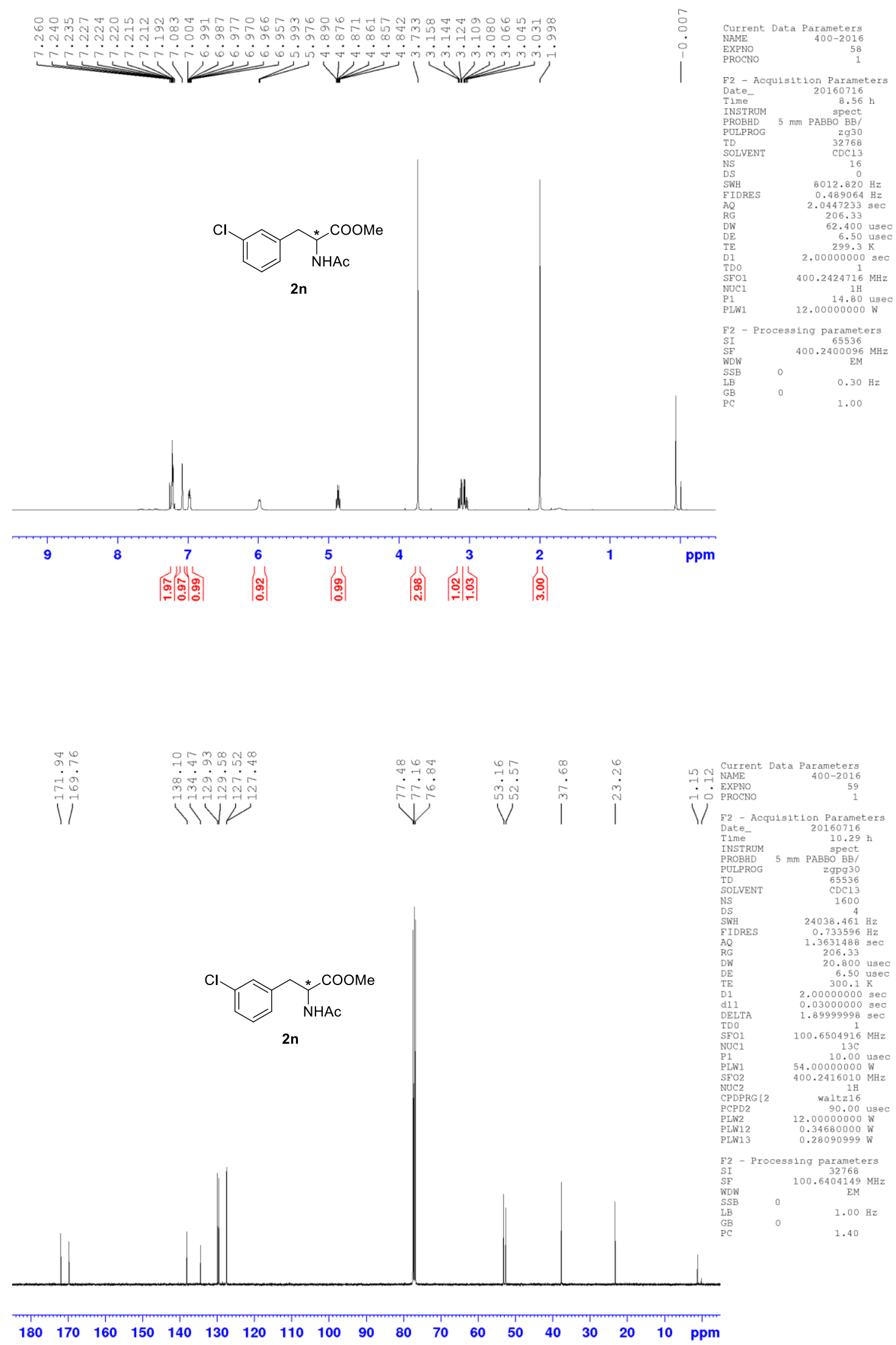
NMR spectra of amide products
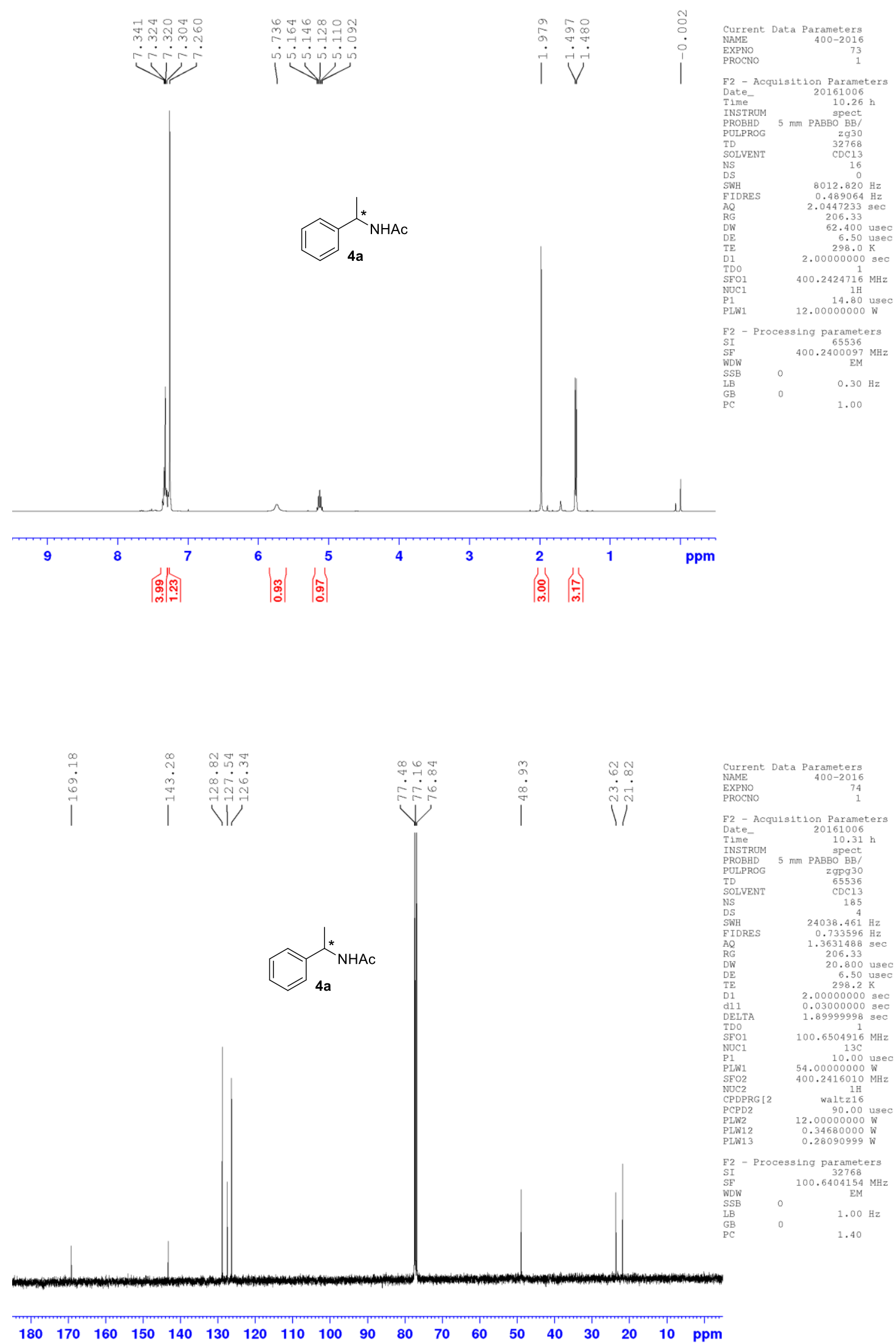

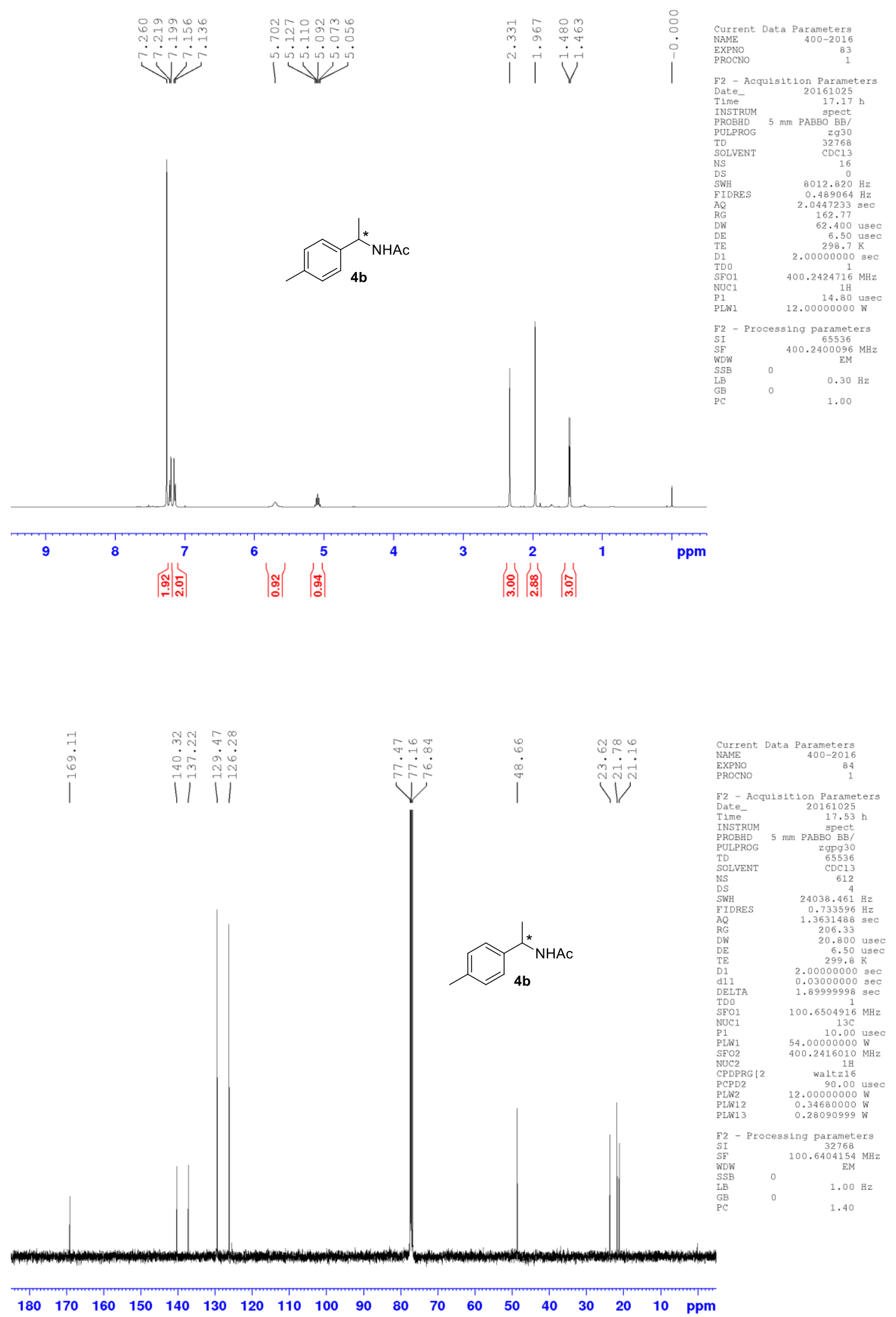

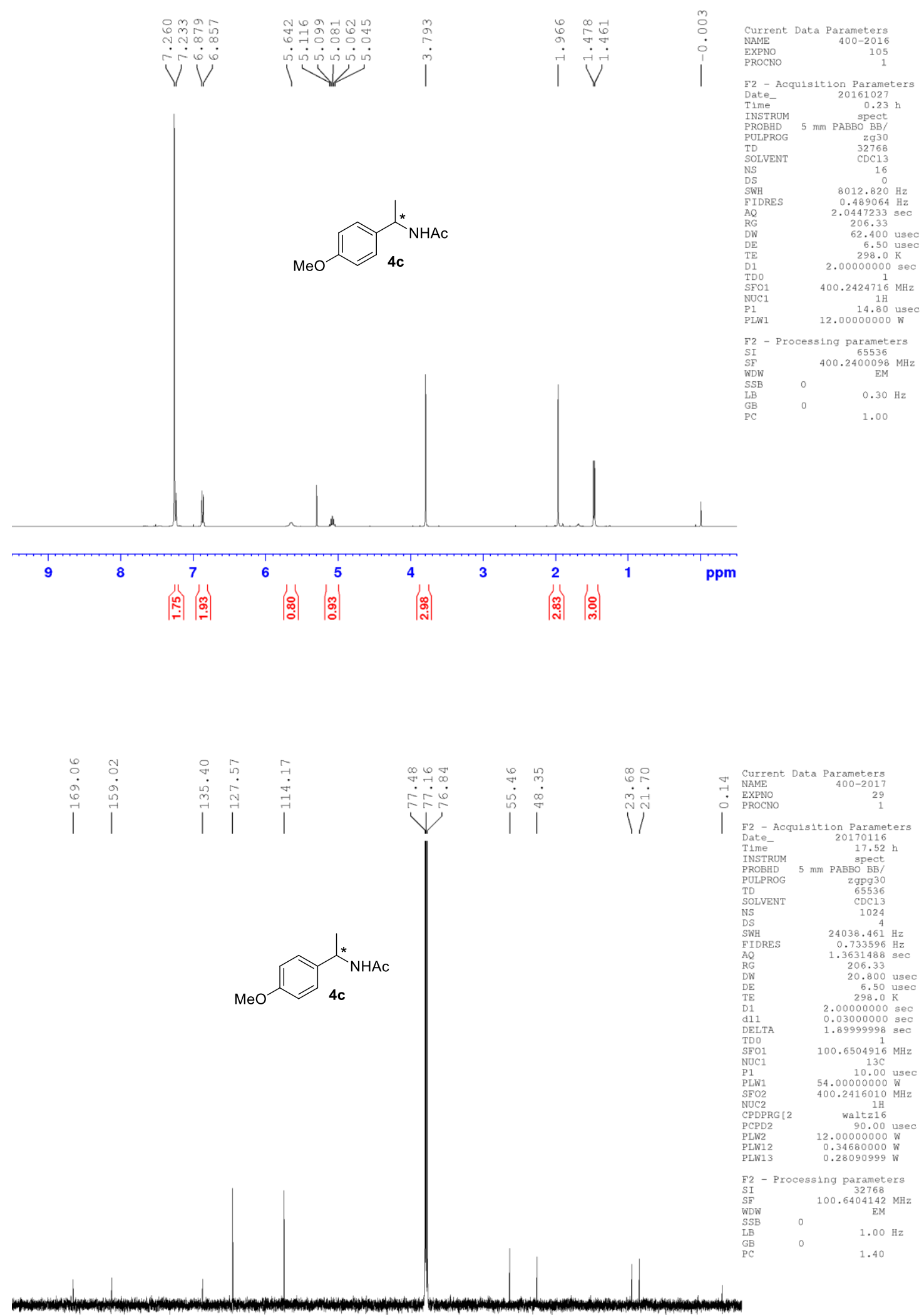

$\begin{array}{lllllllllllllllllll}180 & 170 & 160 & 150 & 140 & 130 & 120 & 110 & 100 & 90 & 80 & 70 & 60 & 50 & 40 & 30 & 20 & 10 & \mathrm{ppm}\end{array}$ 

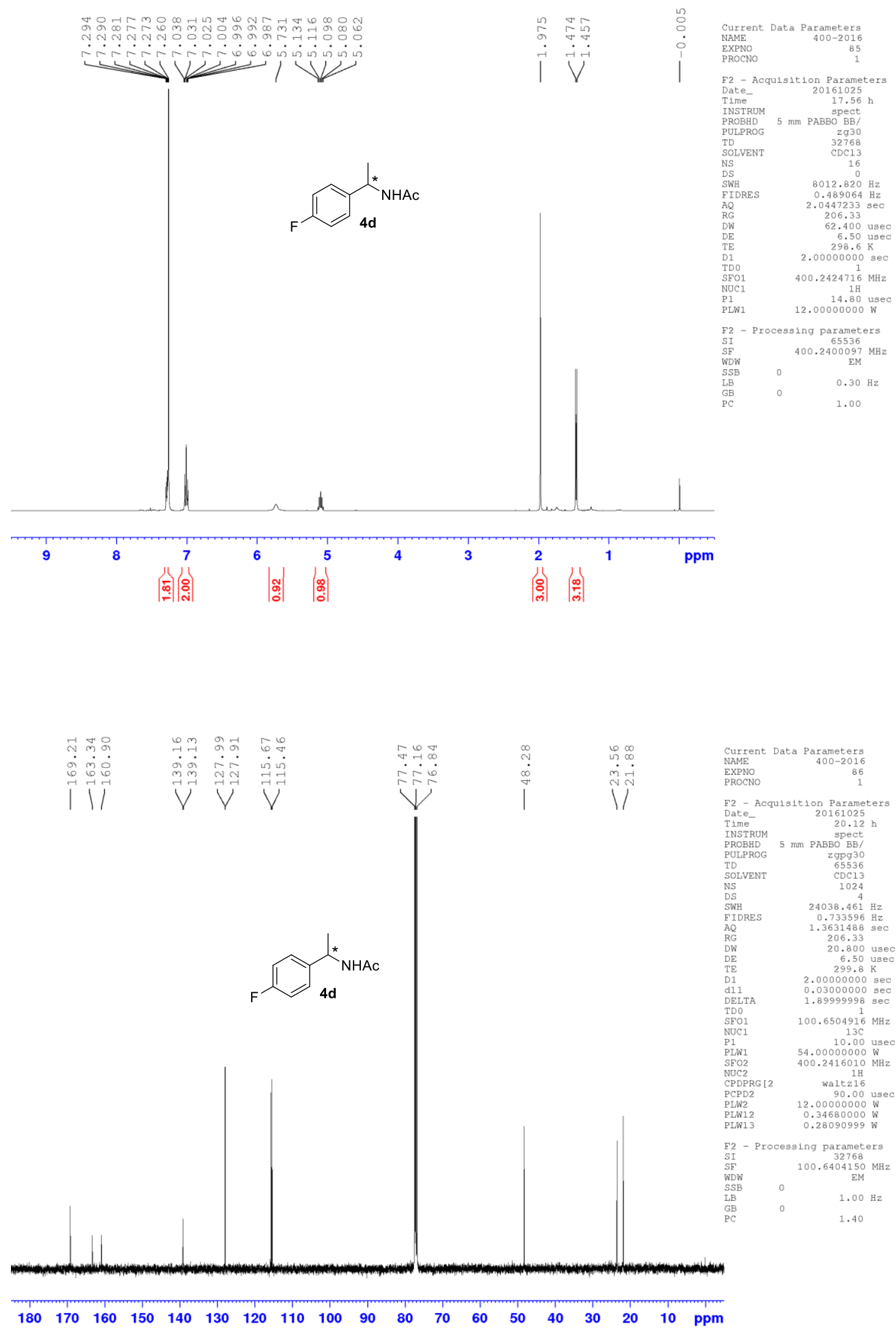

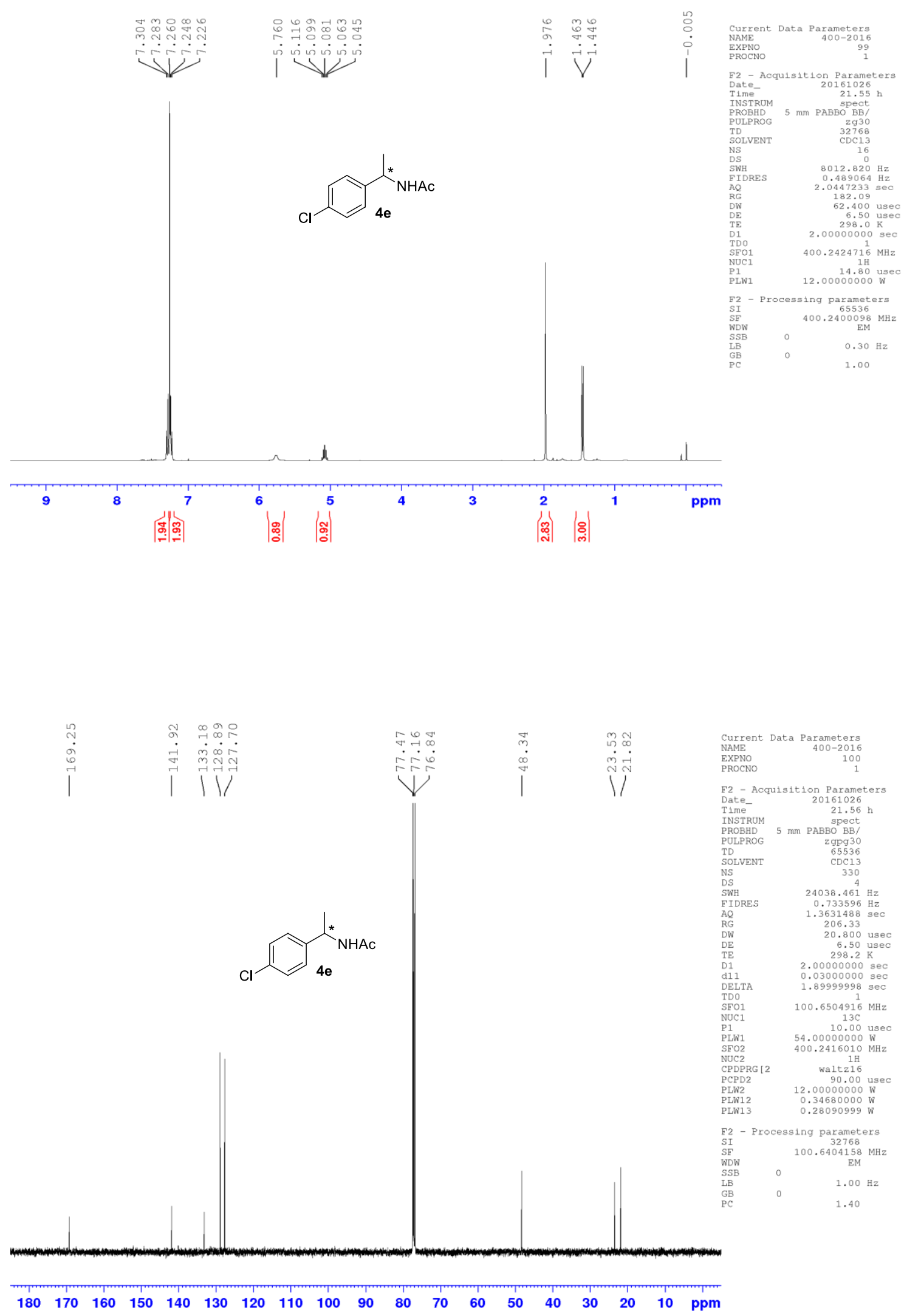

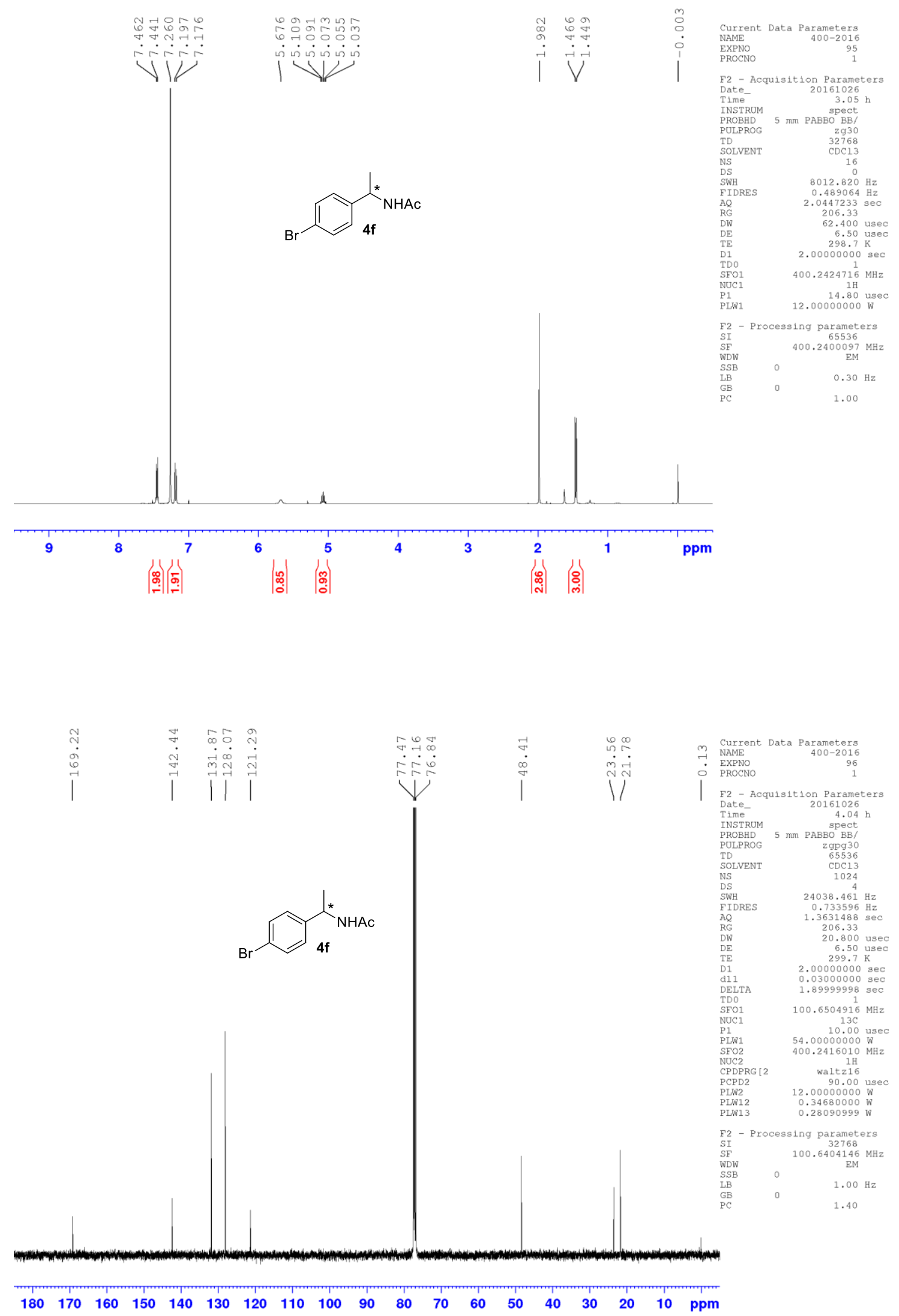

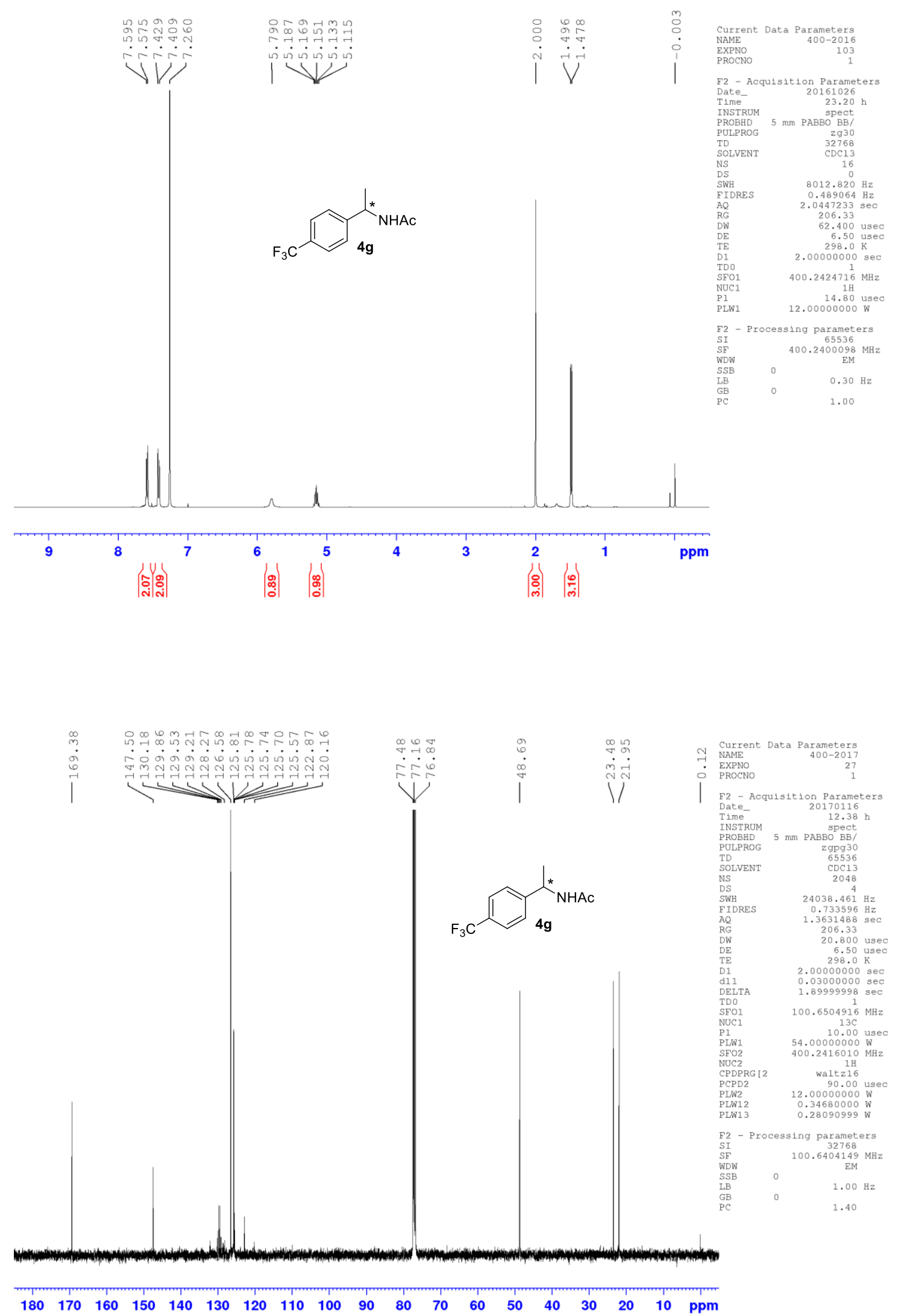

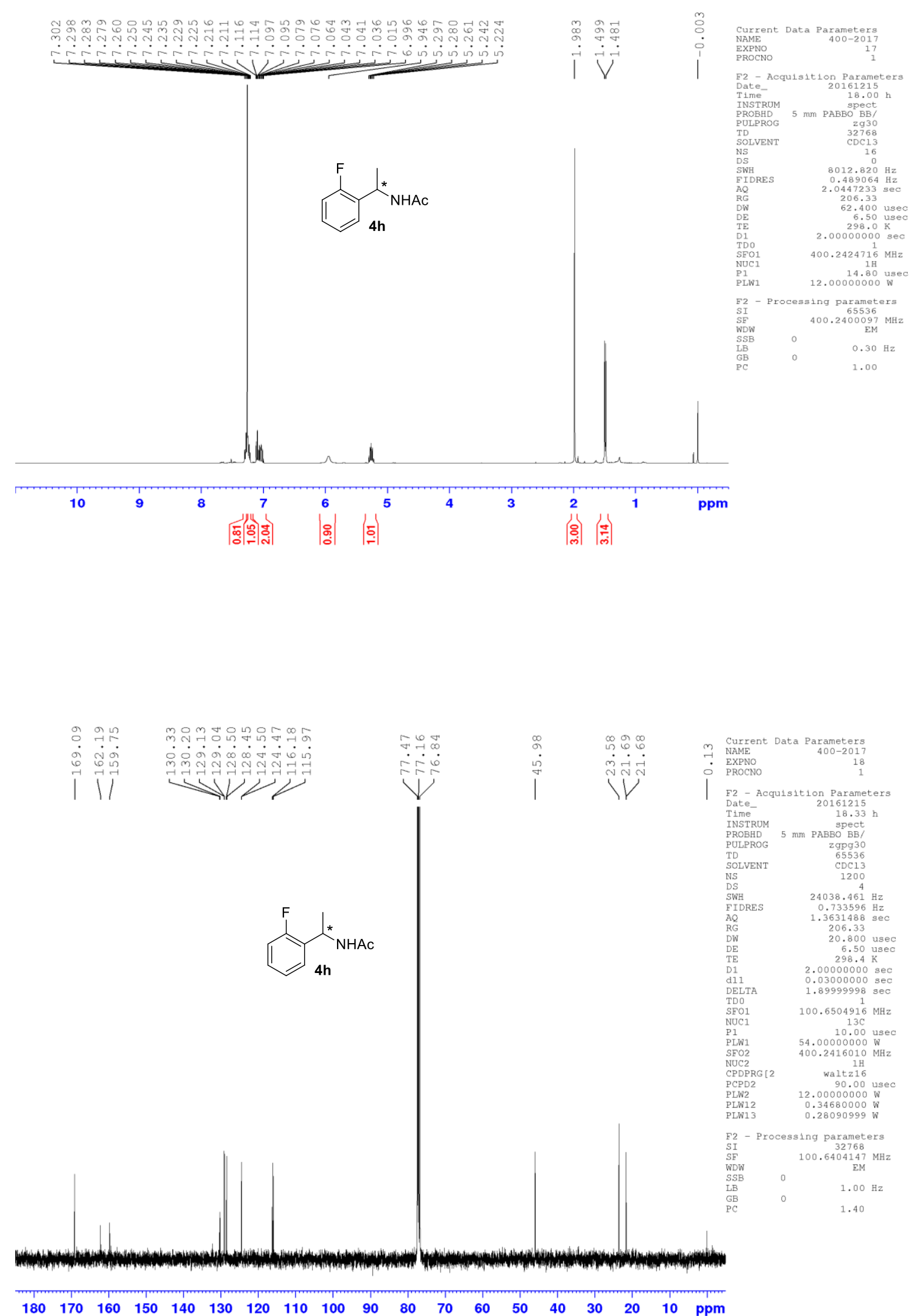

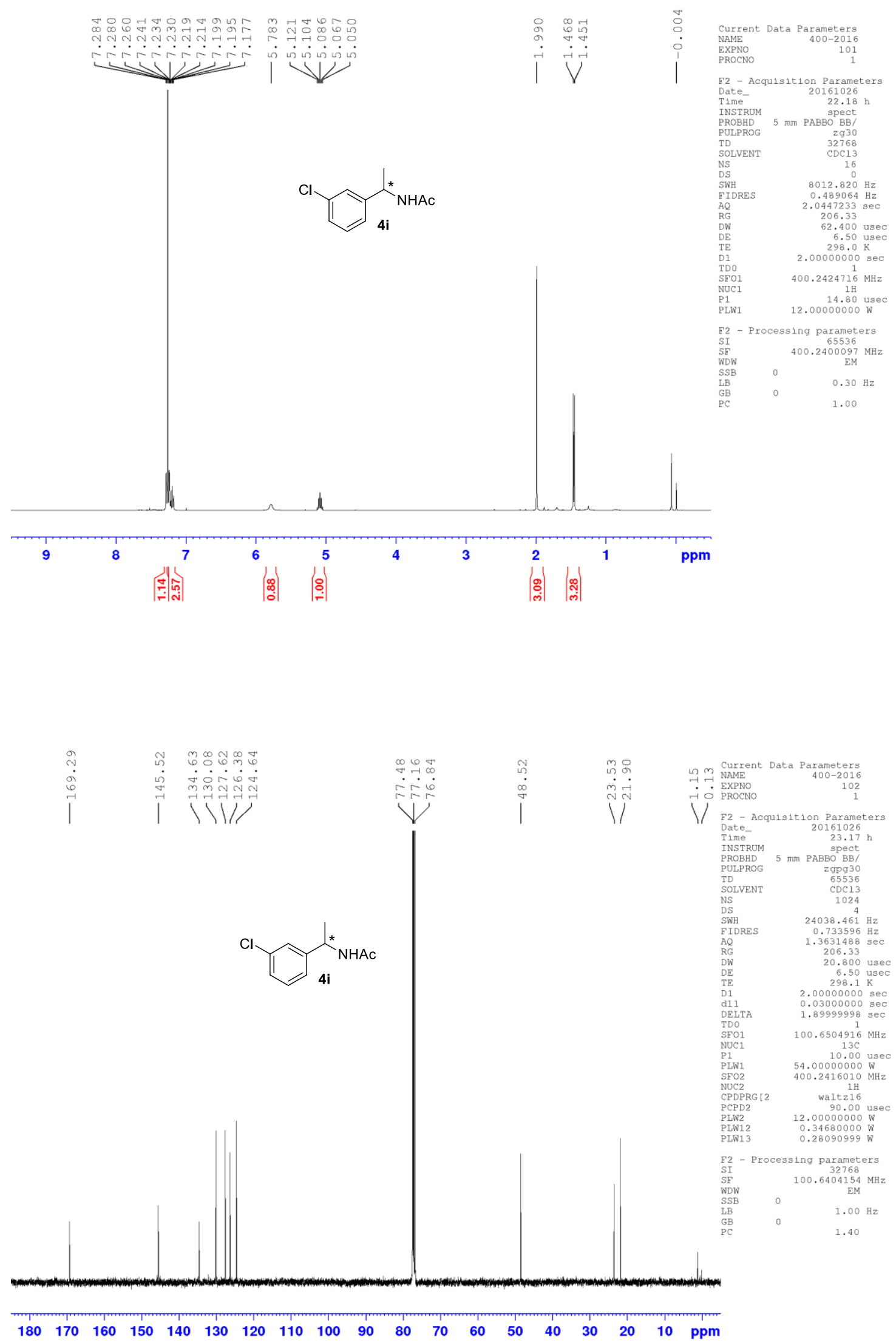

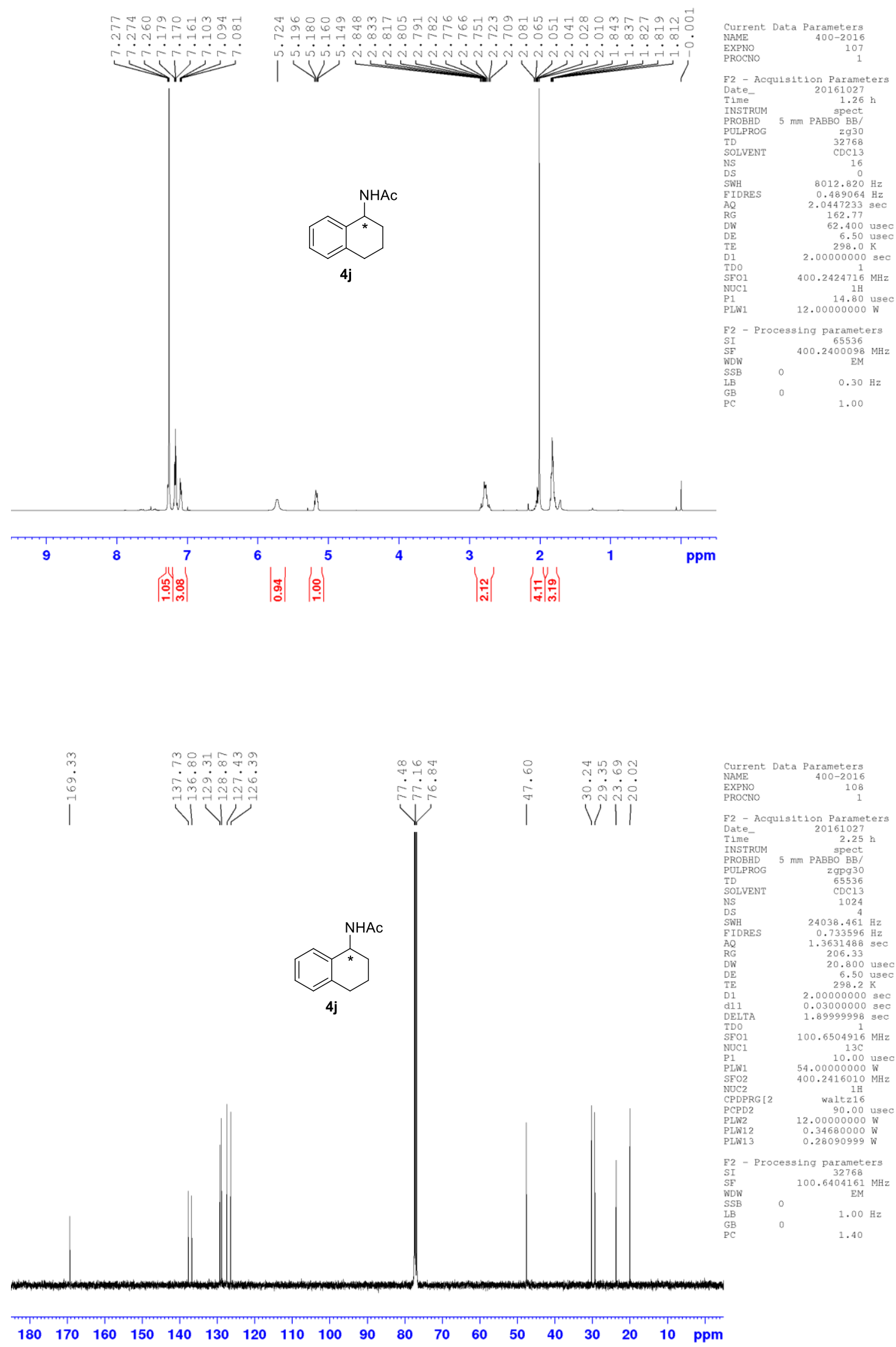
NMR spectra of branched aldehydes

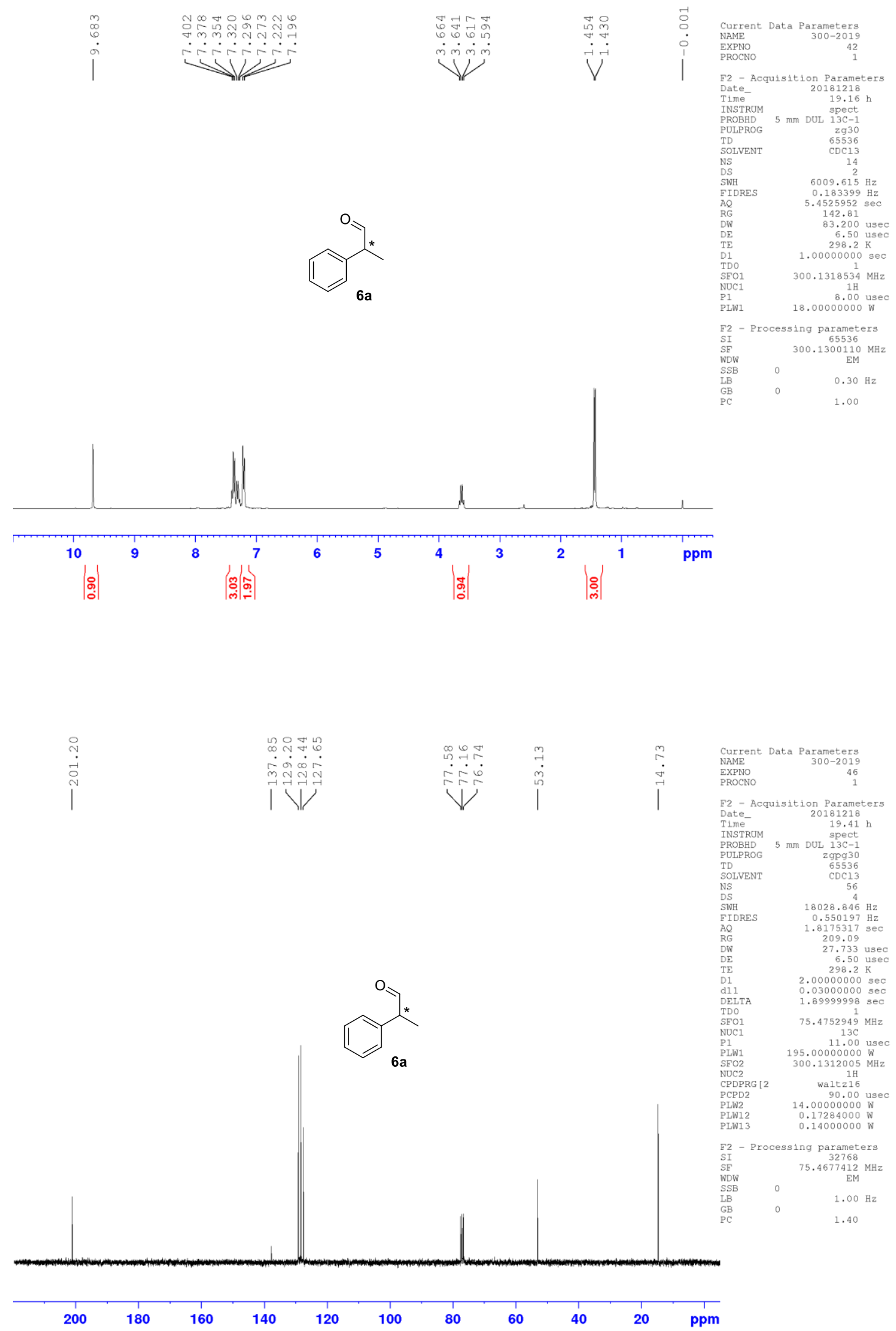



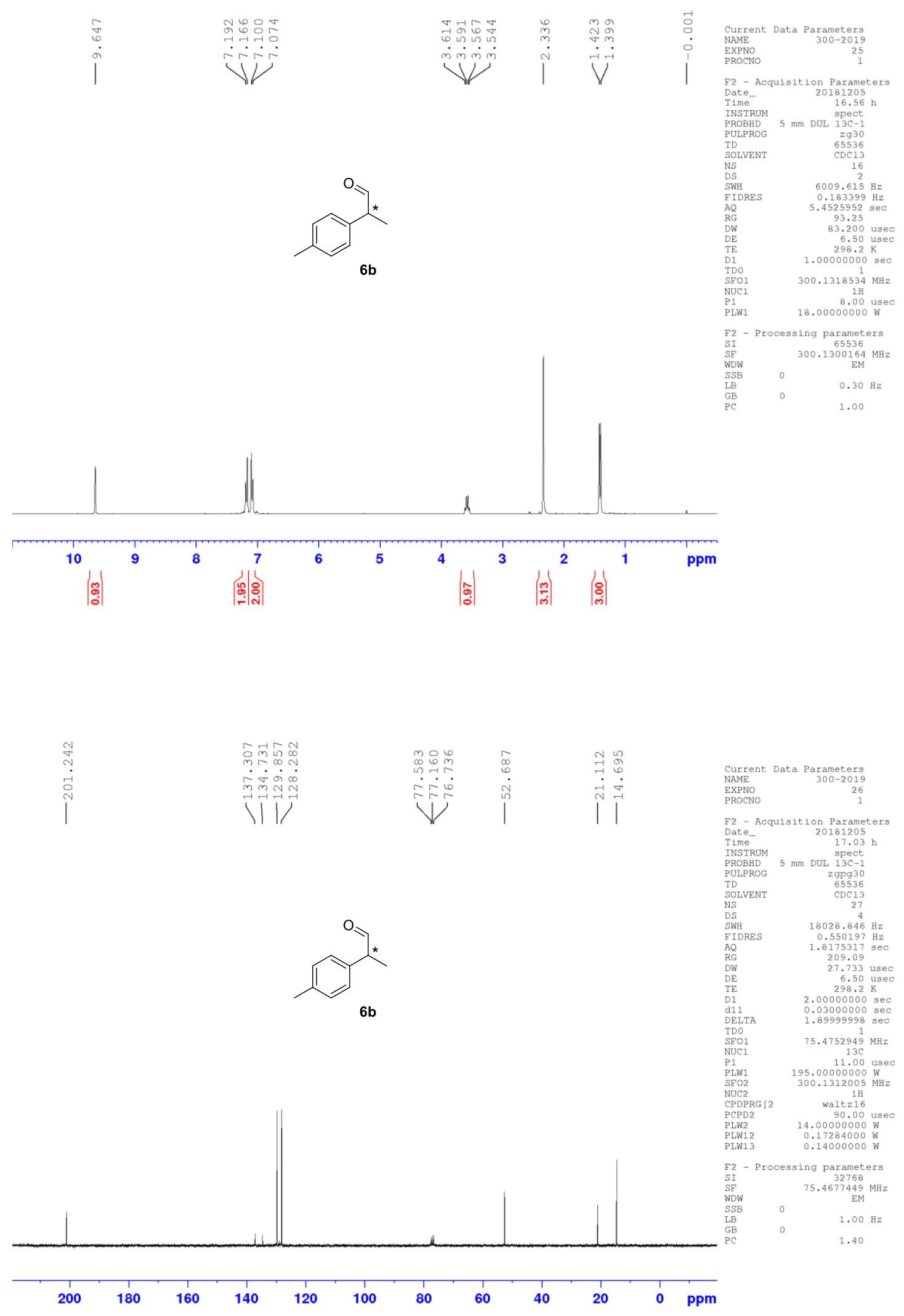


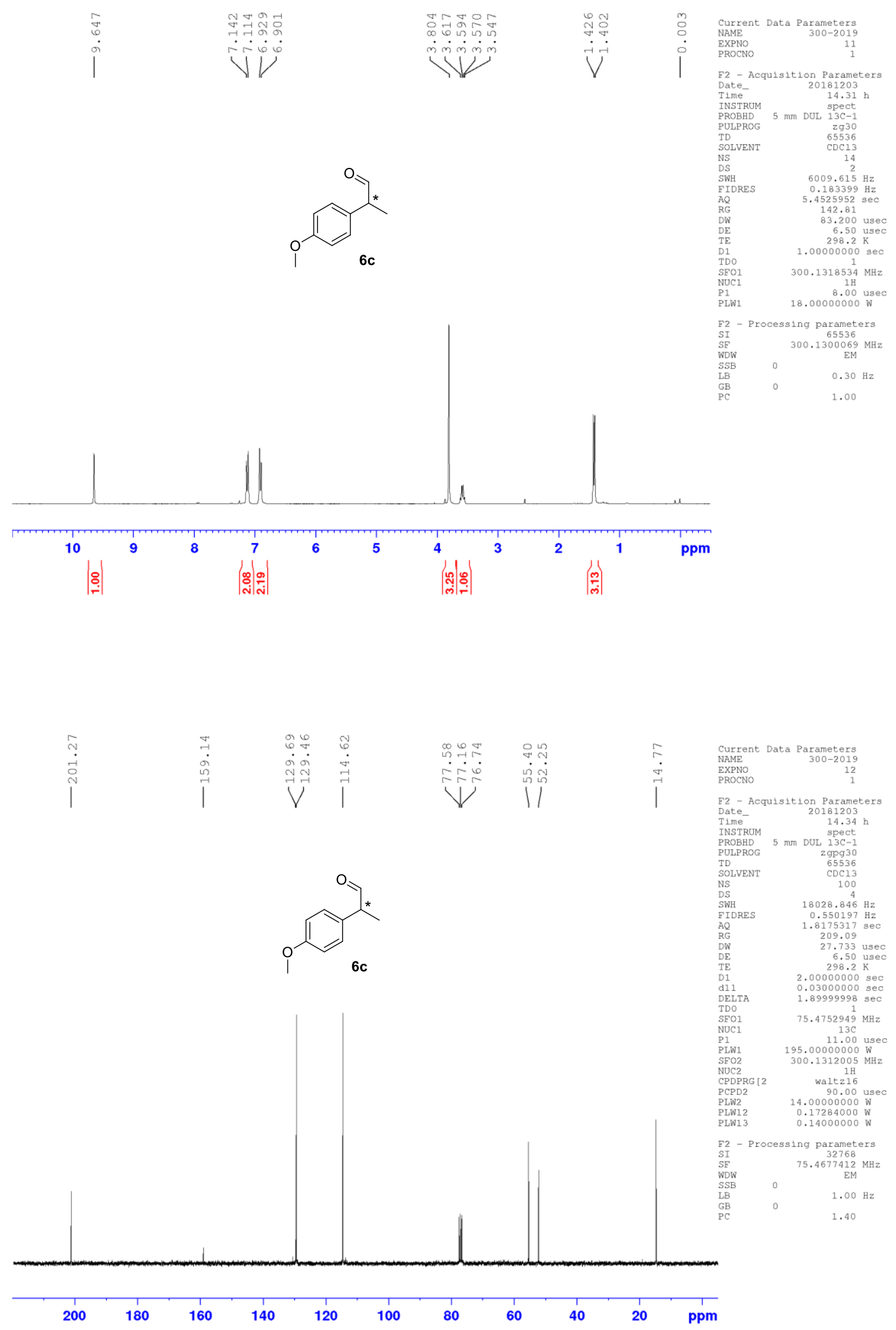




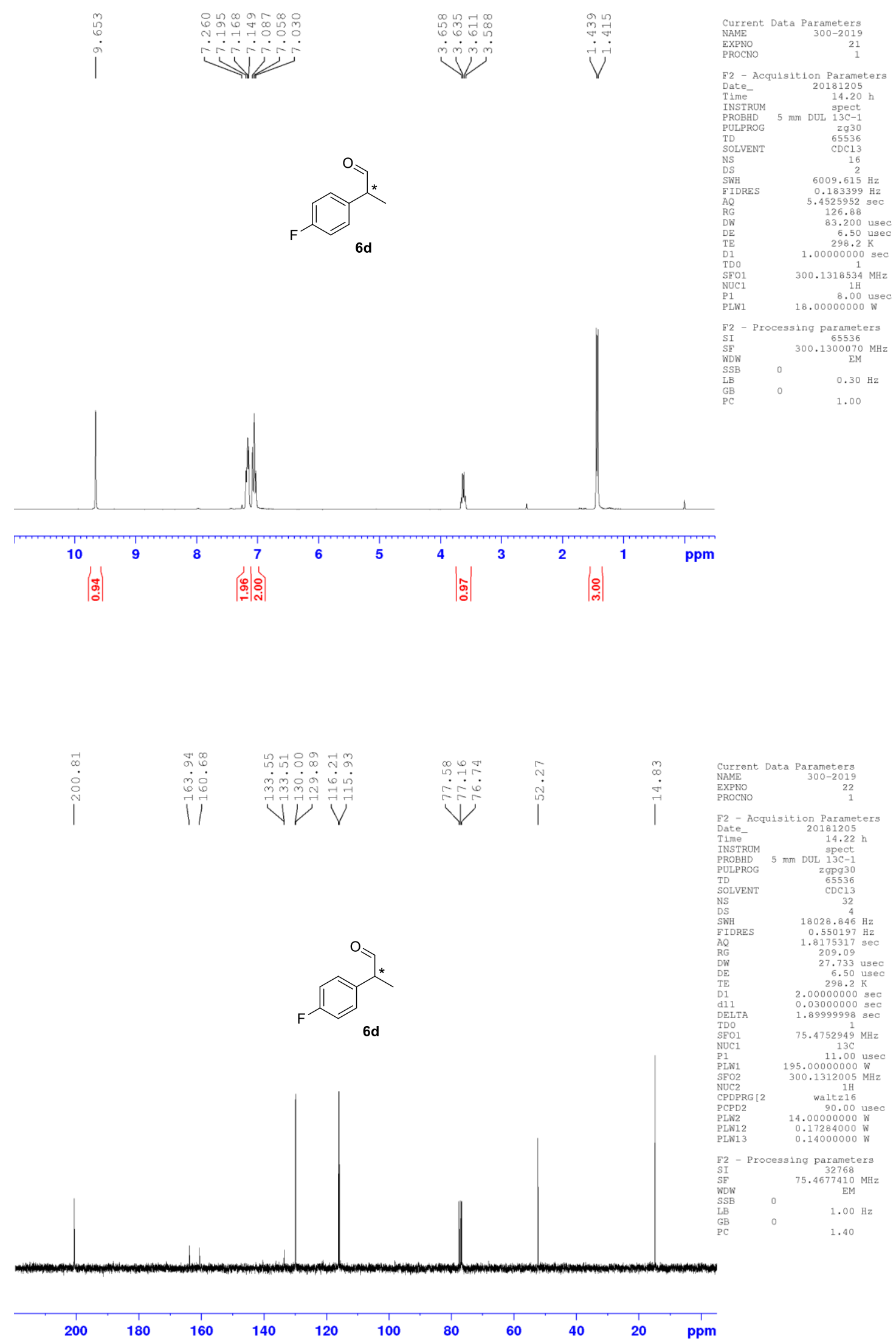




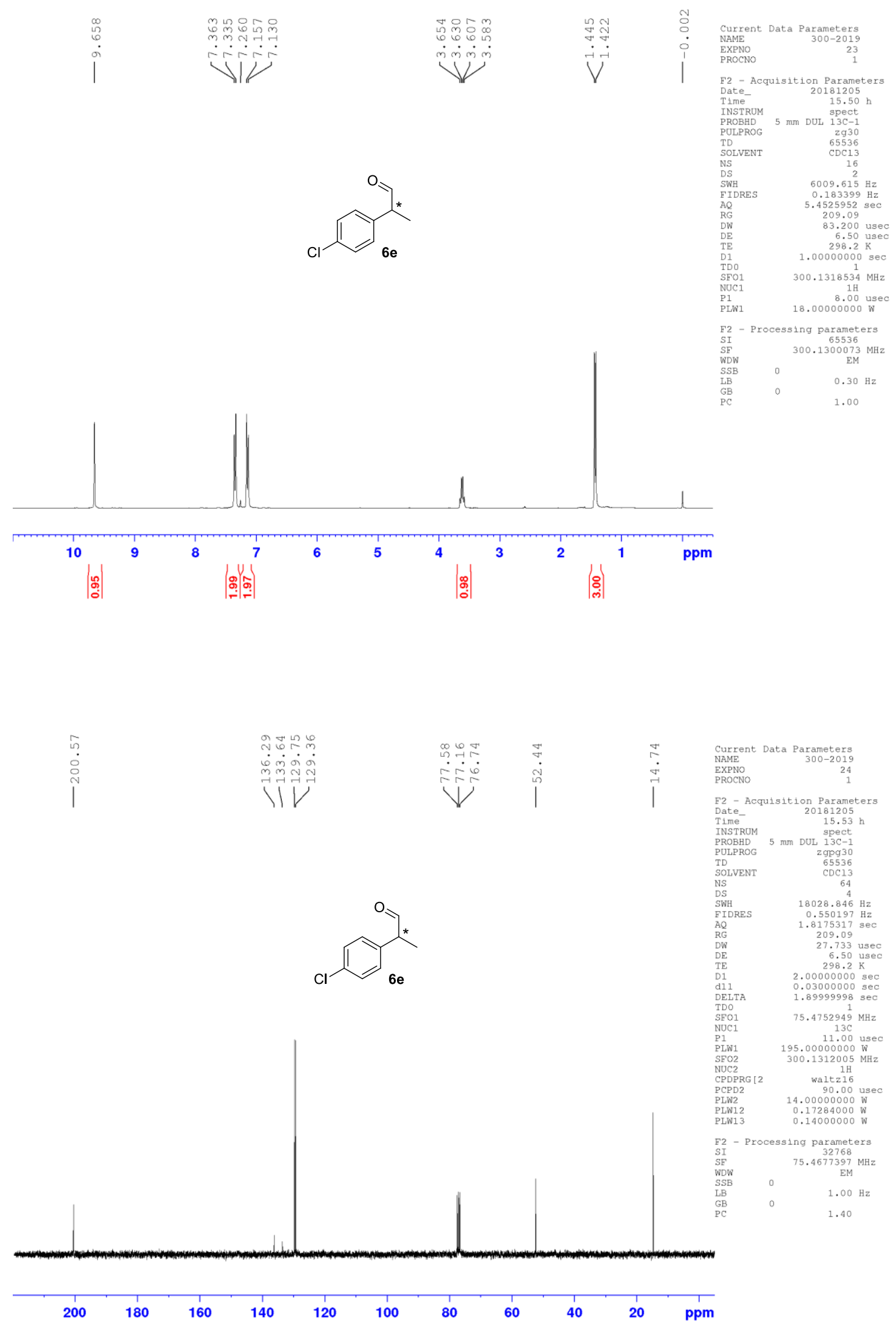




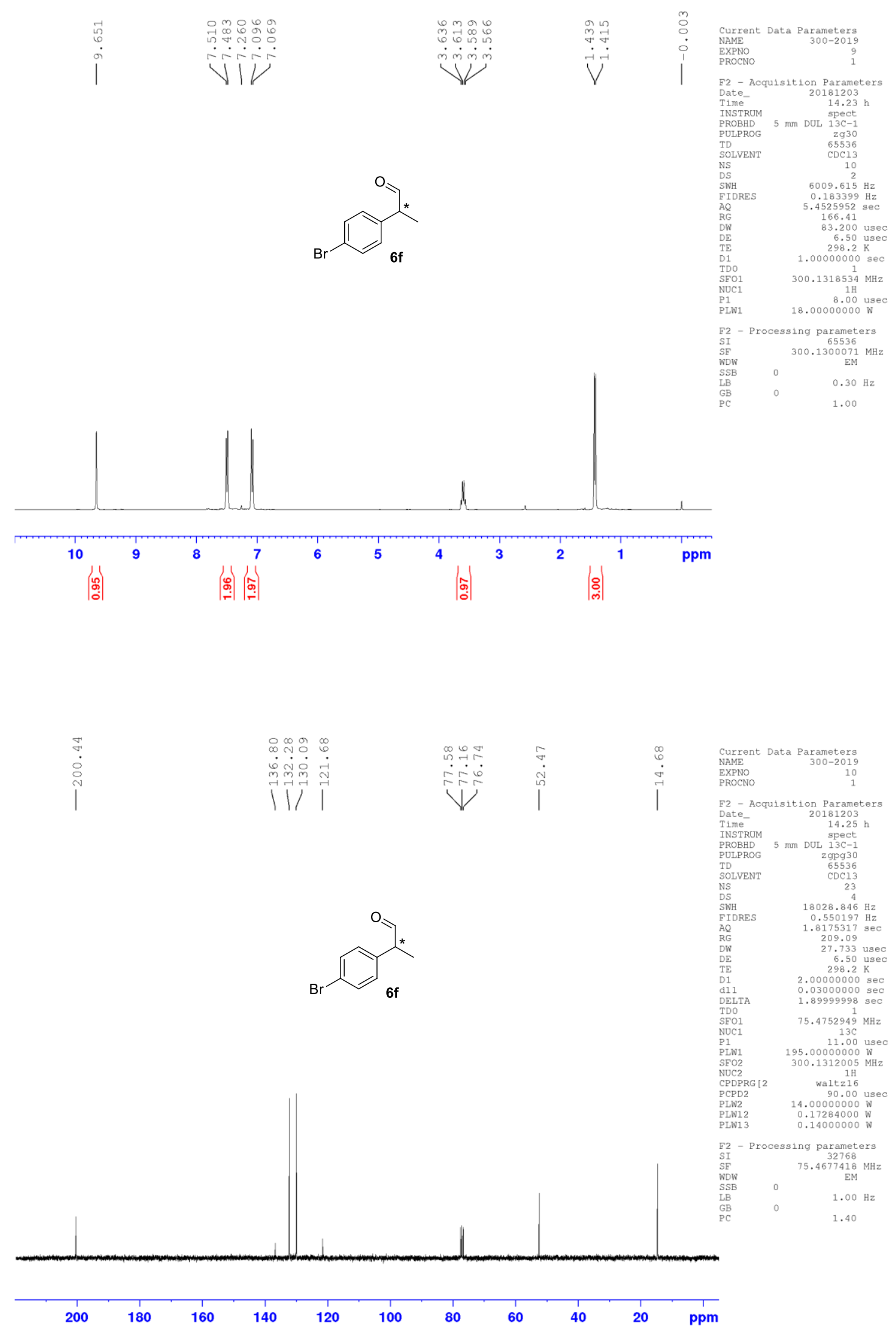




\section{NMR spectra of linear aldehydes}
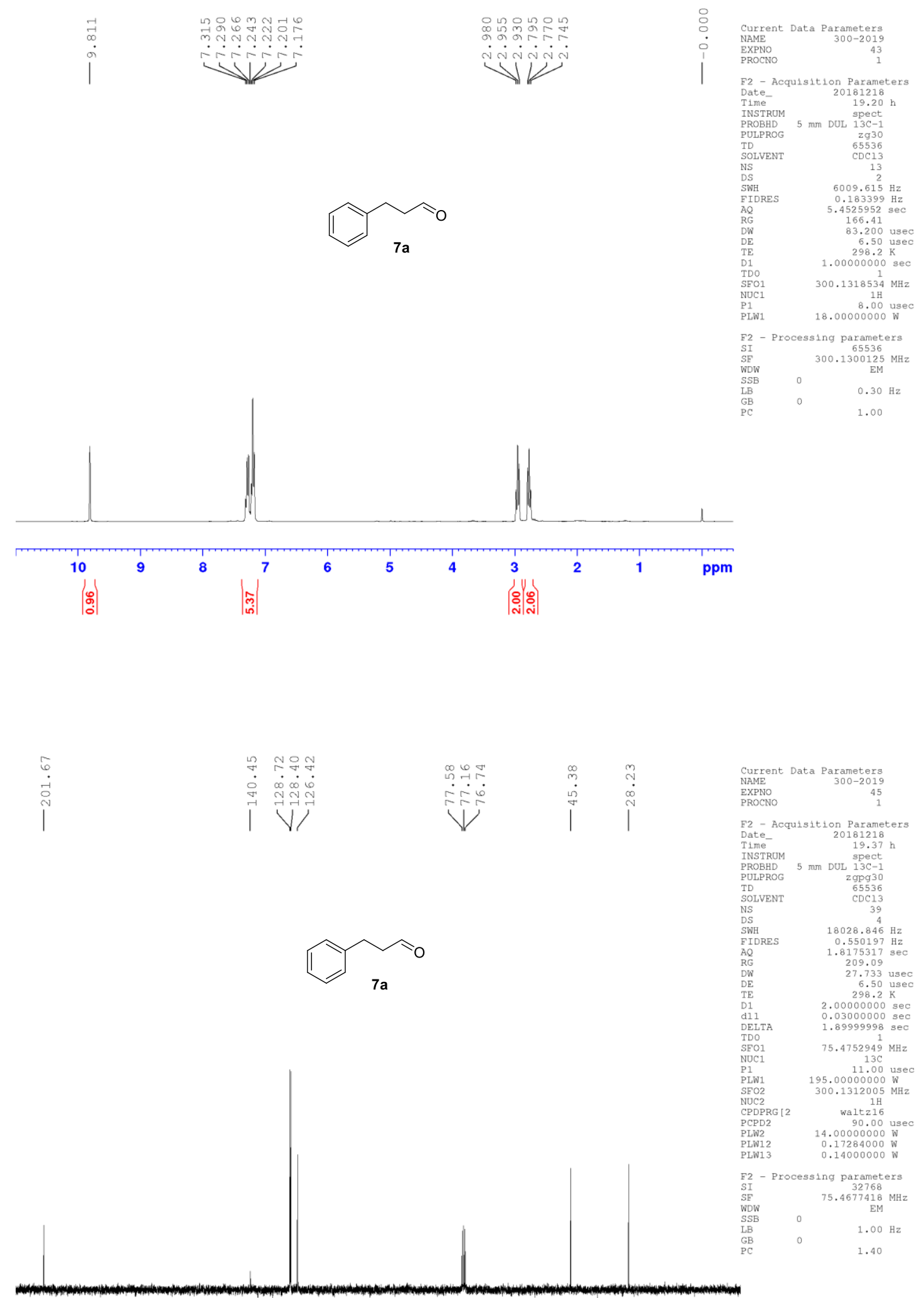


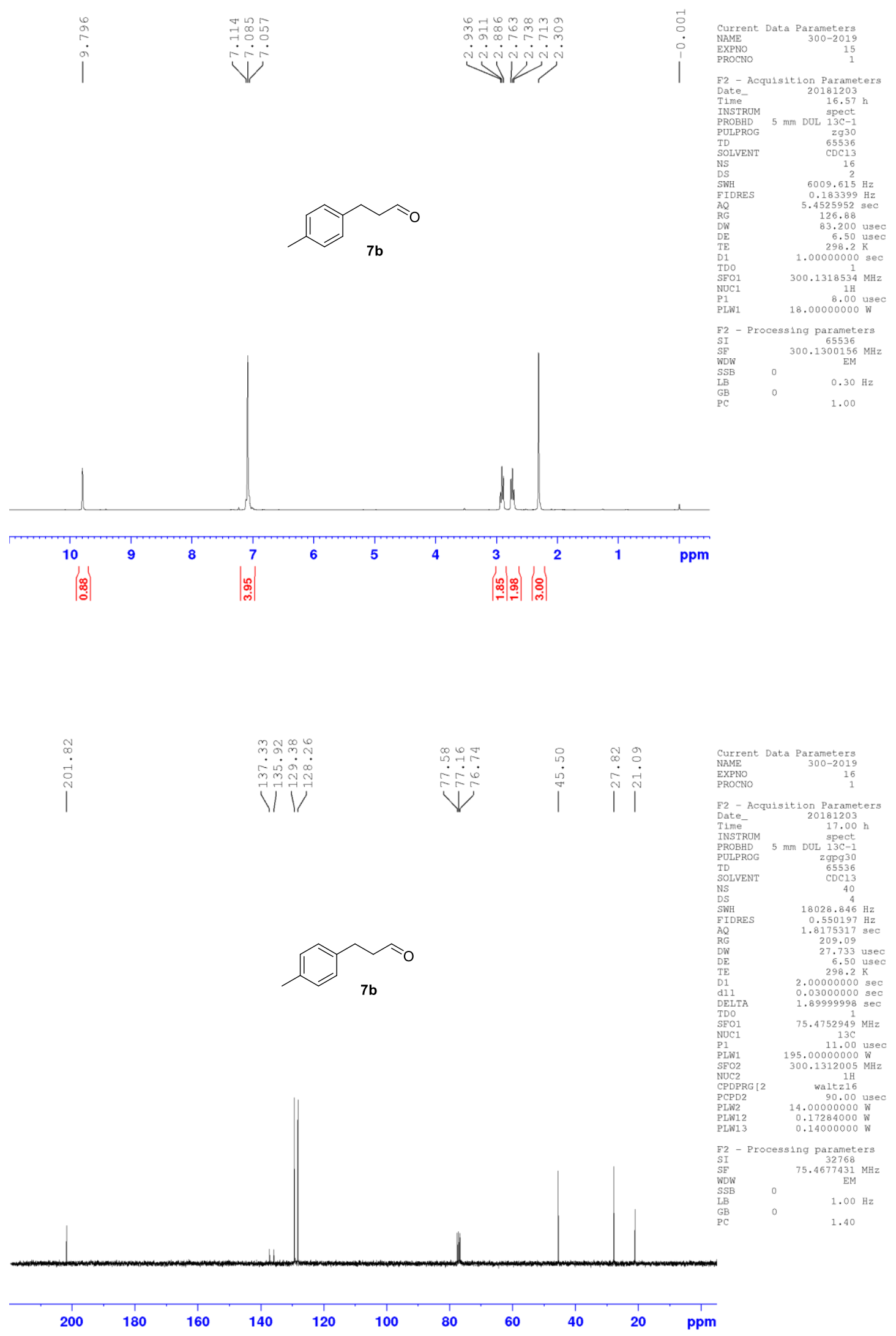




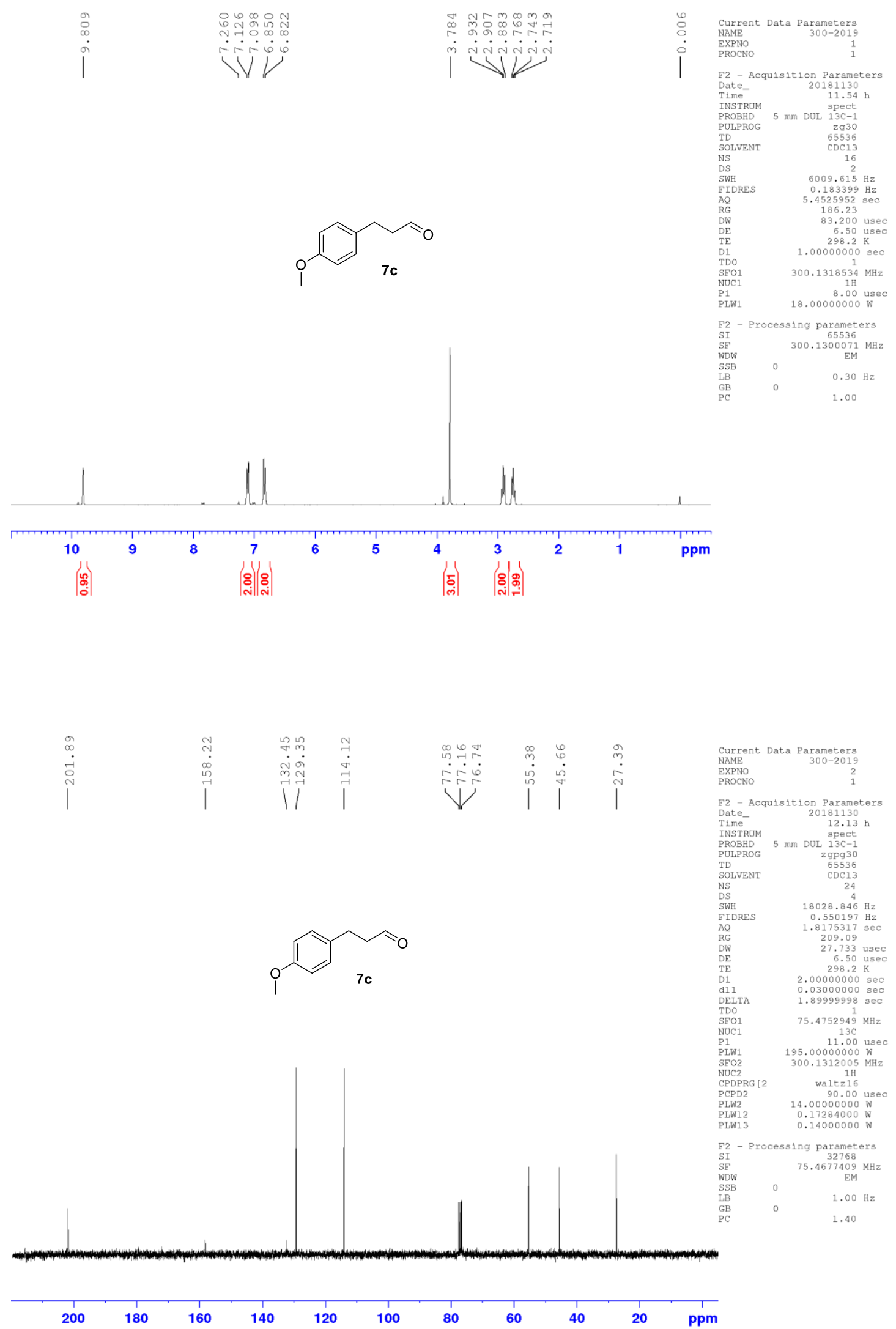




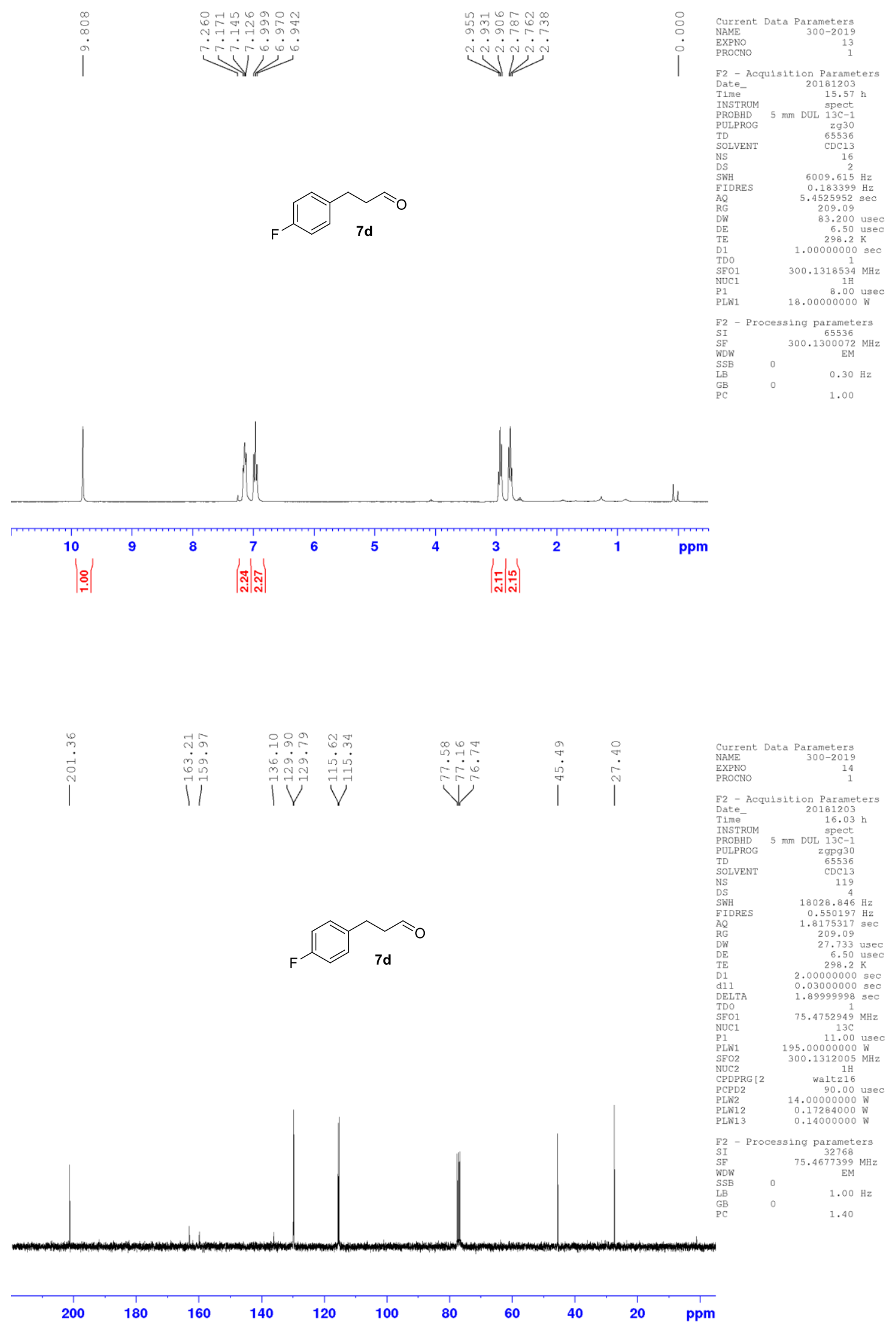




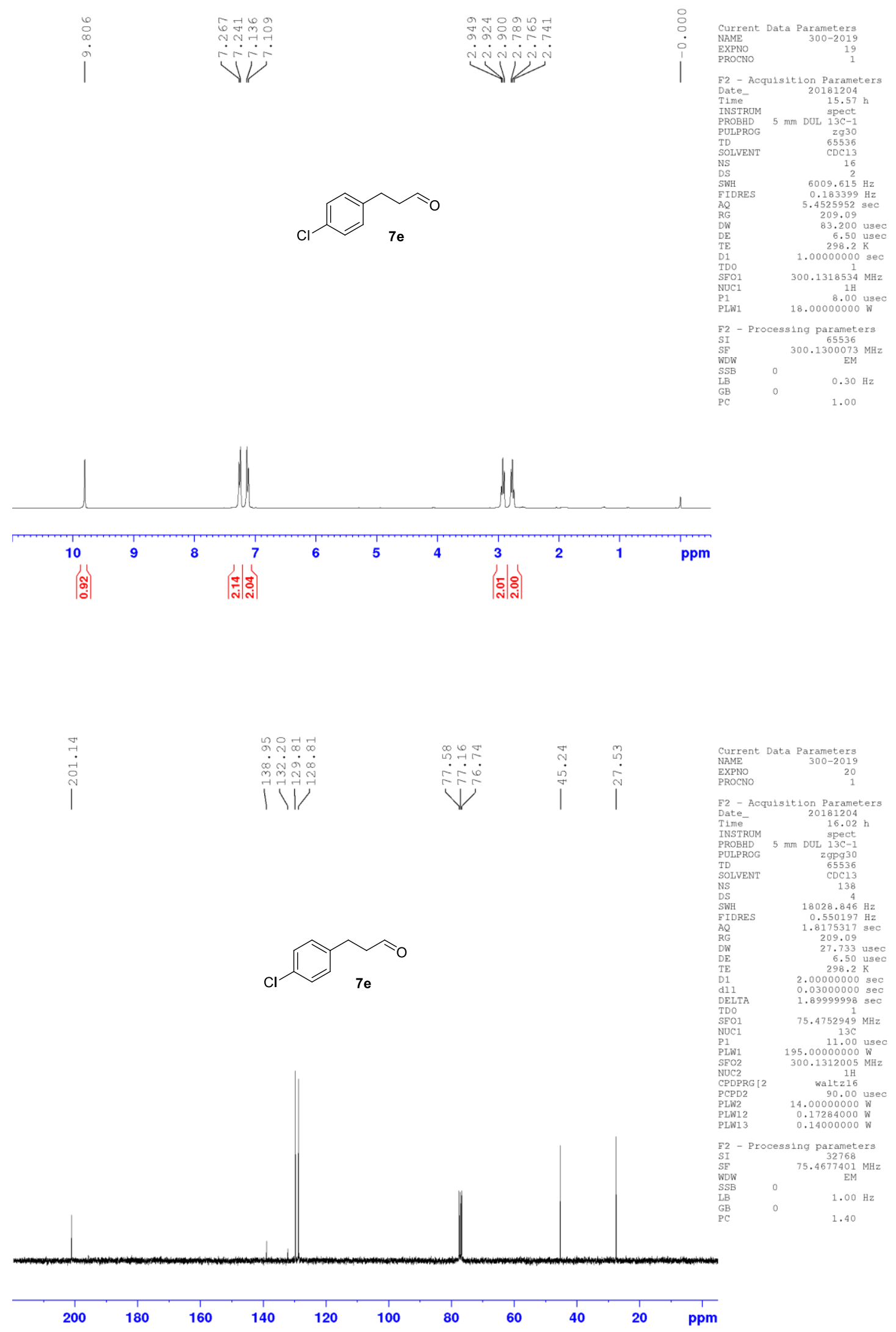




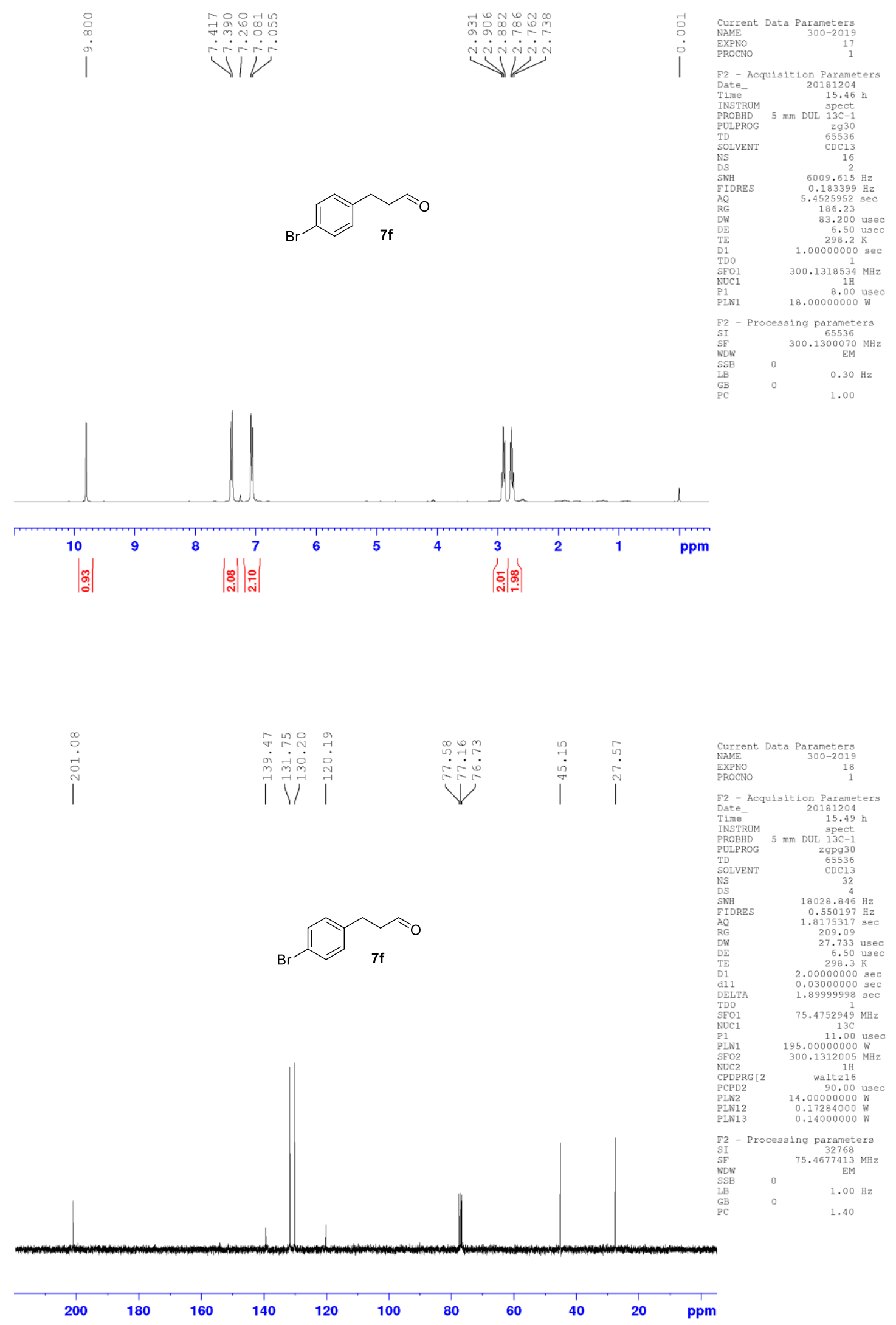




\section{Copy of GC and HPLC spectra}

Asymmetric hydrogenation of dehydroamino acid esters using $(S, S)$-L1
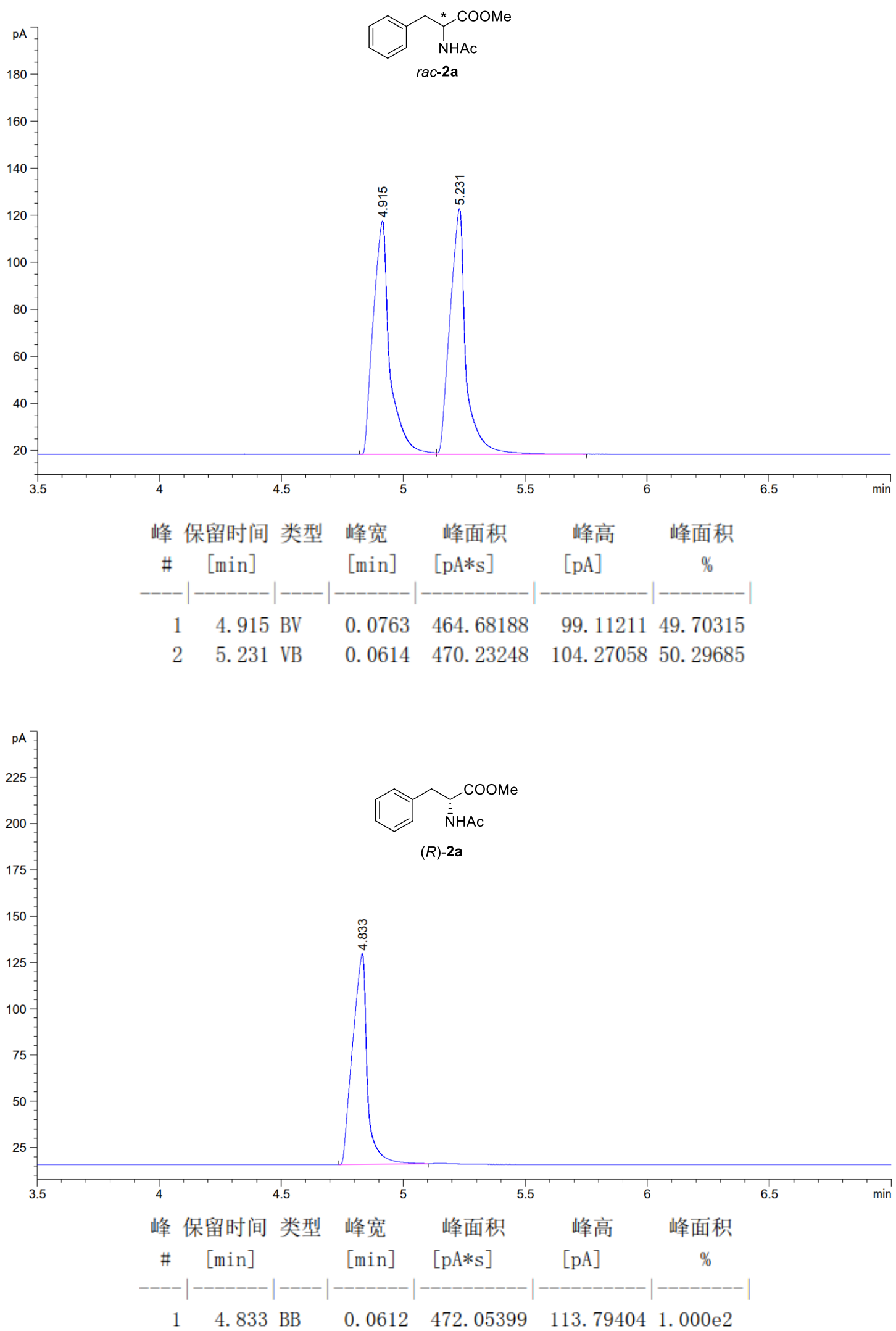

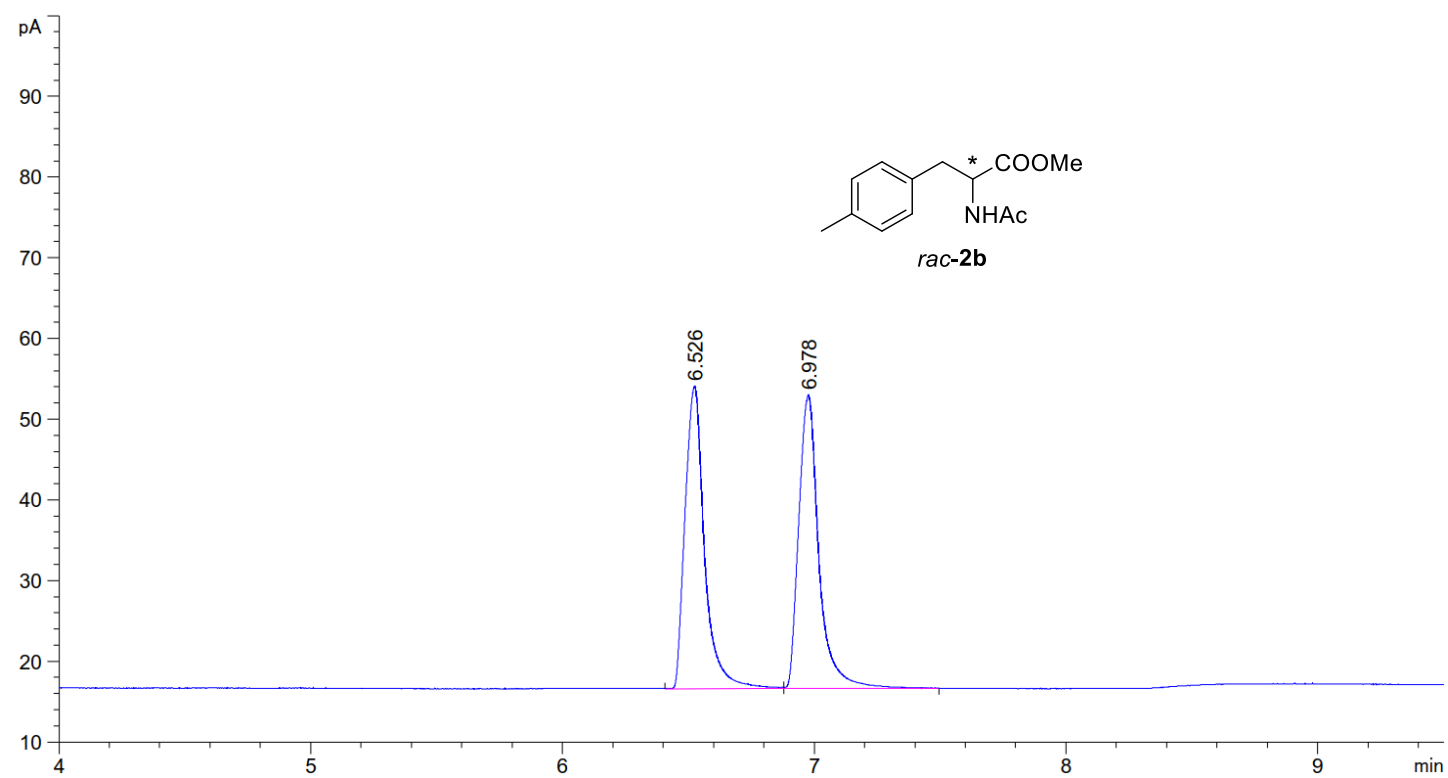

峰 保留时间 类型 峰宽 峰面积峰高 峰面积

$\begin{array}{cccccc}\# & {[\mathrm{~min}]} & {[\mathrm{min}]} & {[\mathrm{pA} * \mathrm{~s}]} & {[\mathrm{pA}]} & \% \\ -1 & 6.526 \mathrm{BV} & 0.0835 & 201.27539 & 37.47362 & 49.92747 \\ 2 & 6.978 \text { VB } & 0.0848 & 201.86021 & 36.32772 & 50.07253\end{array}$

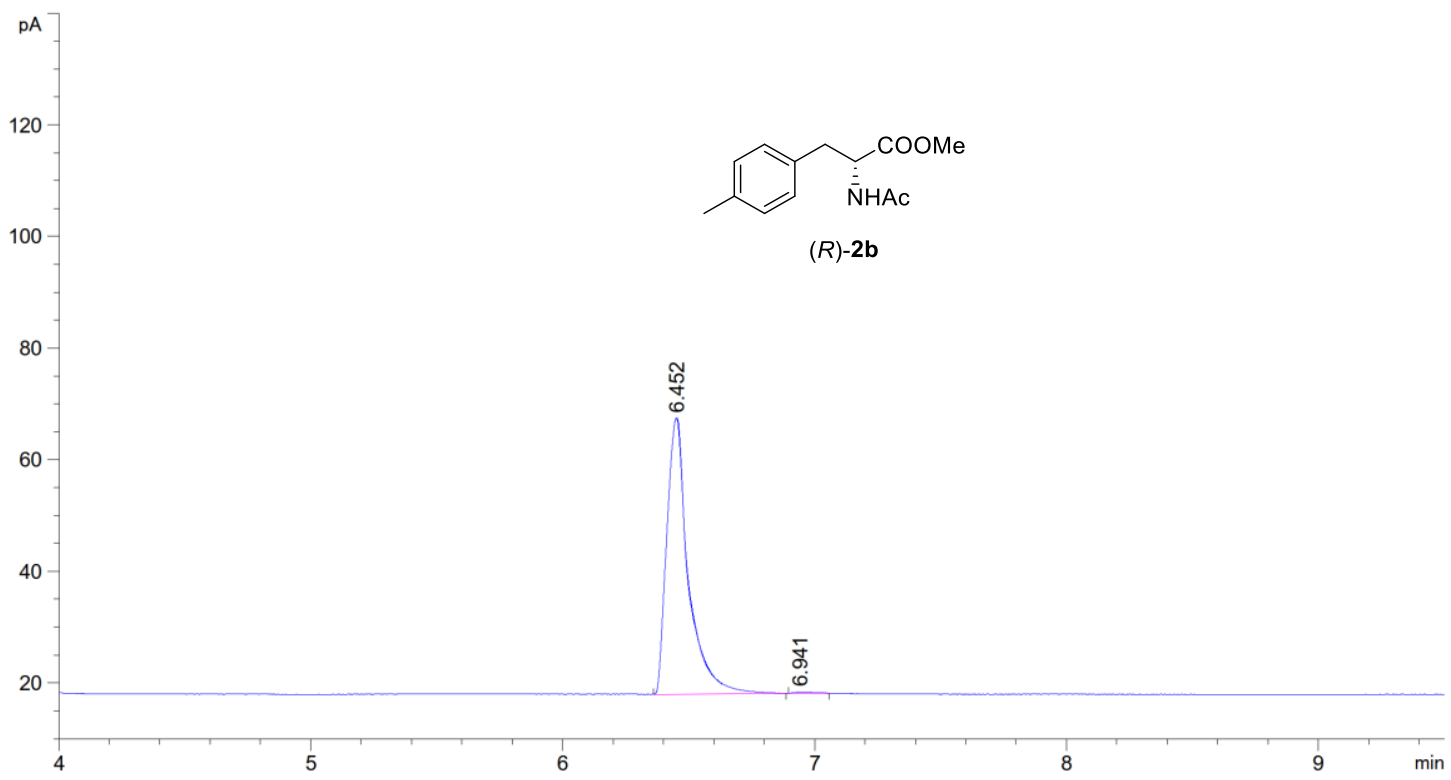

峰 保留时间 类型 峰宽 峰面积峰高 峰面积

\begin{tabular}{cccccc}
$\#$ & {$[\mathrm{~min}]$} & {$[\mathrm{min}]$} & {$[\mathrm{pA} * \mathrm{~s}]$} & {$[\mathrm{pA}]$} & $\%$ \\
\hline 1 & 6.452 BB & 0.0887 & 294.71326 & 49.48335 & 99.59355 \\
2 & 6.941 MM & 0.0861 & 1.20274 & $2.32843 \mathrm{e}^{-1}$ & 0.40645
\end{tabular}



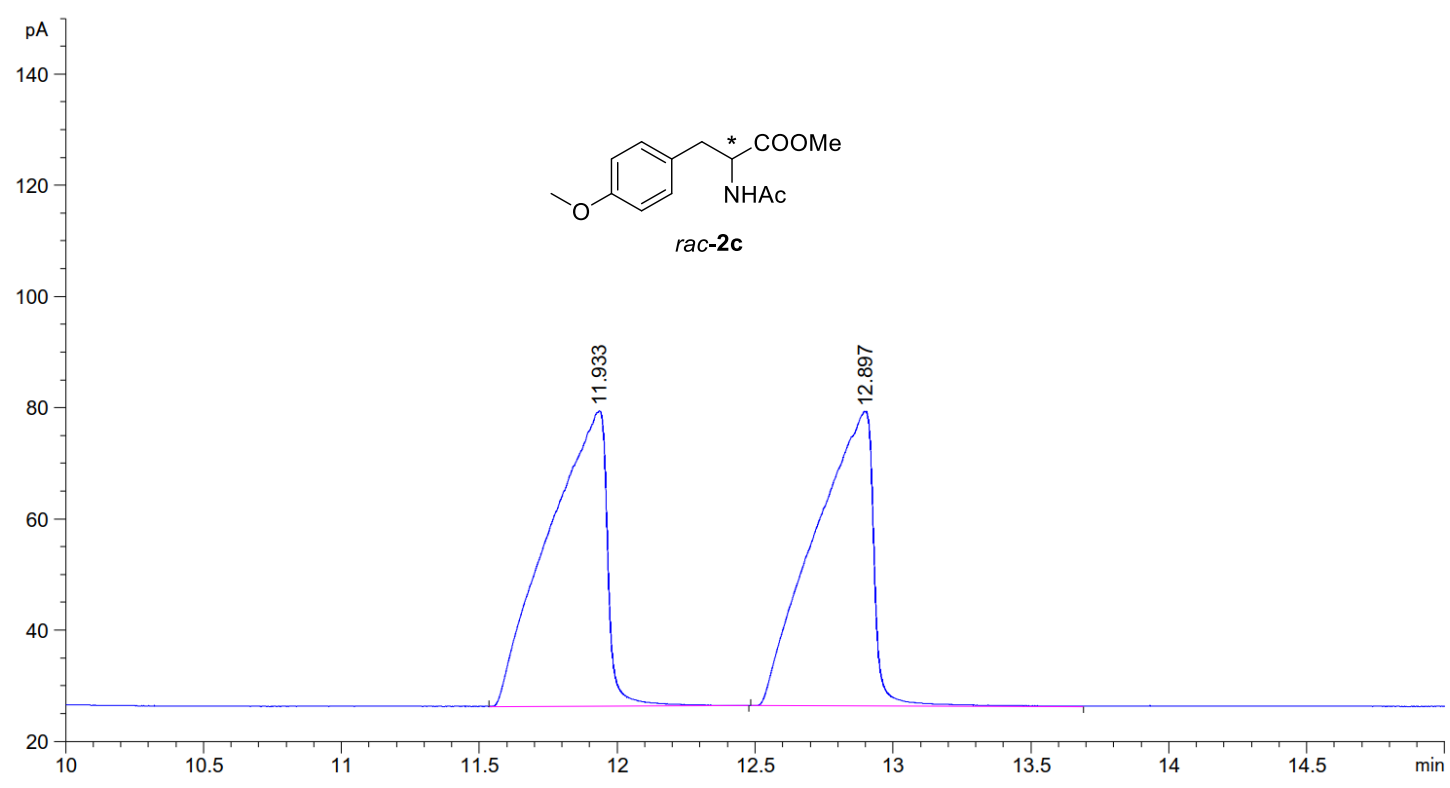

峰 保留时间 类型 峰宽 峰面积峰高 峰面积

\begin{tabular}{cccccc}
$\#$ & {$[\mathrm{~min}]$} & {$[\mathrm{min}]$} & {$[\mathrm{pA} * \mathrm{~s}]$} & {$[\mathrm{pA}]$} & $\%$ \\
\hdashline & $11.933 \mathrm{BB}$ & 0.1866 & 783.05774 & 53.04936 & 49.94987 \\
2 & $12.897 \mathrm{BB}$ & 0.1907 & 784.62964 & 52.87024 & 50.05013
\end{tabular}

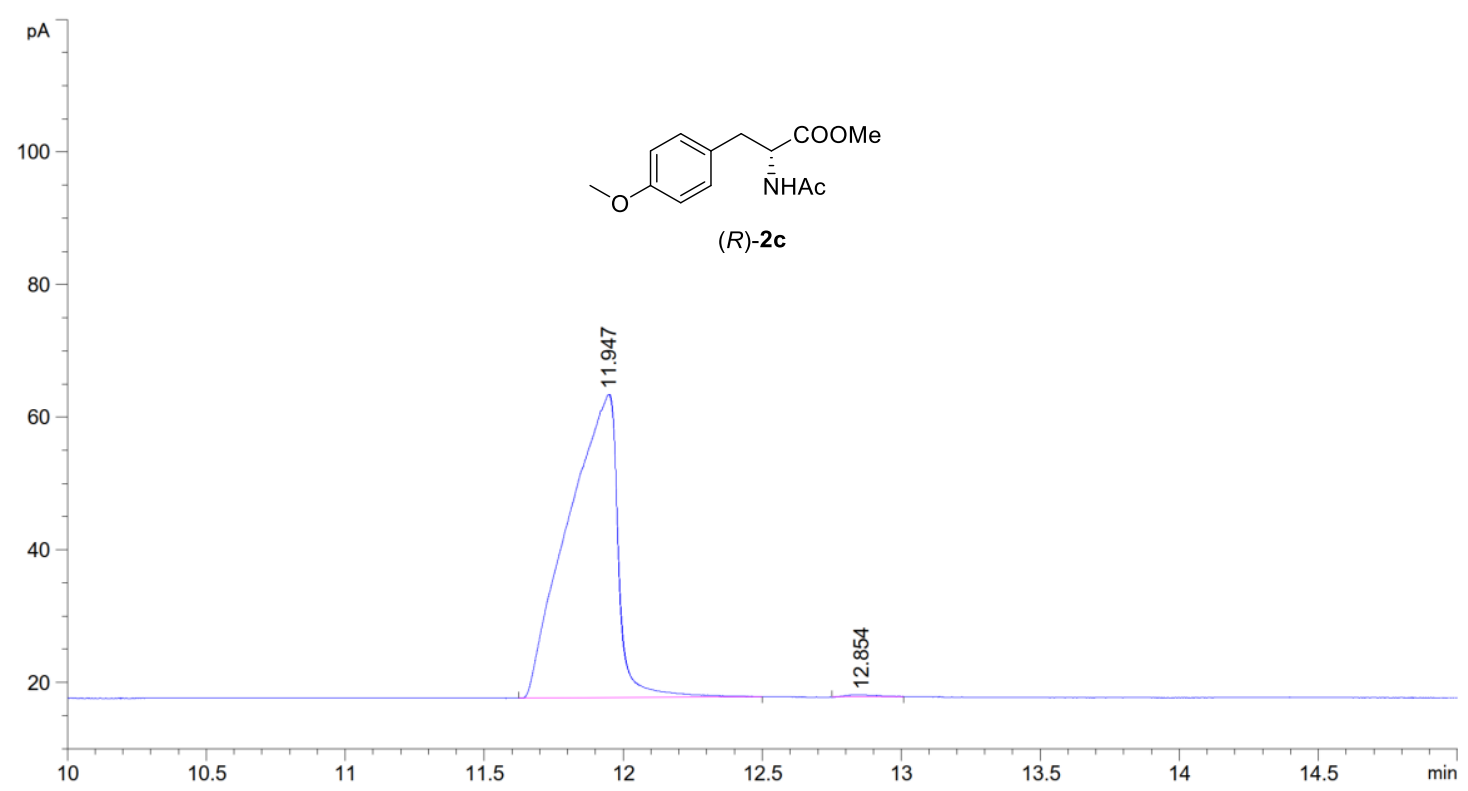

峰 保留时间类型 峰宽 峰面积峰高峰面积

\begin{tabular}{cccccc}
$\#$ & {$[\mathrm{~min}]$} & {$[\mathrm{min}]$} & {$[\mathrm{pA}$ s $]$} & {$[\mathrm{pA}]$} & $\%$ \\
\hline 1 & $11.947 \mathrm{BB}$ & 0.1568 & 570.85657 & 45.72928 & 99.50817 \\
2 & $12.854 \mathrm{MM}$ & 0.1400 & 2.82154 & $3.35878 \mathrm{e}^{-1}$ & 0.49183
\end{tabular}



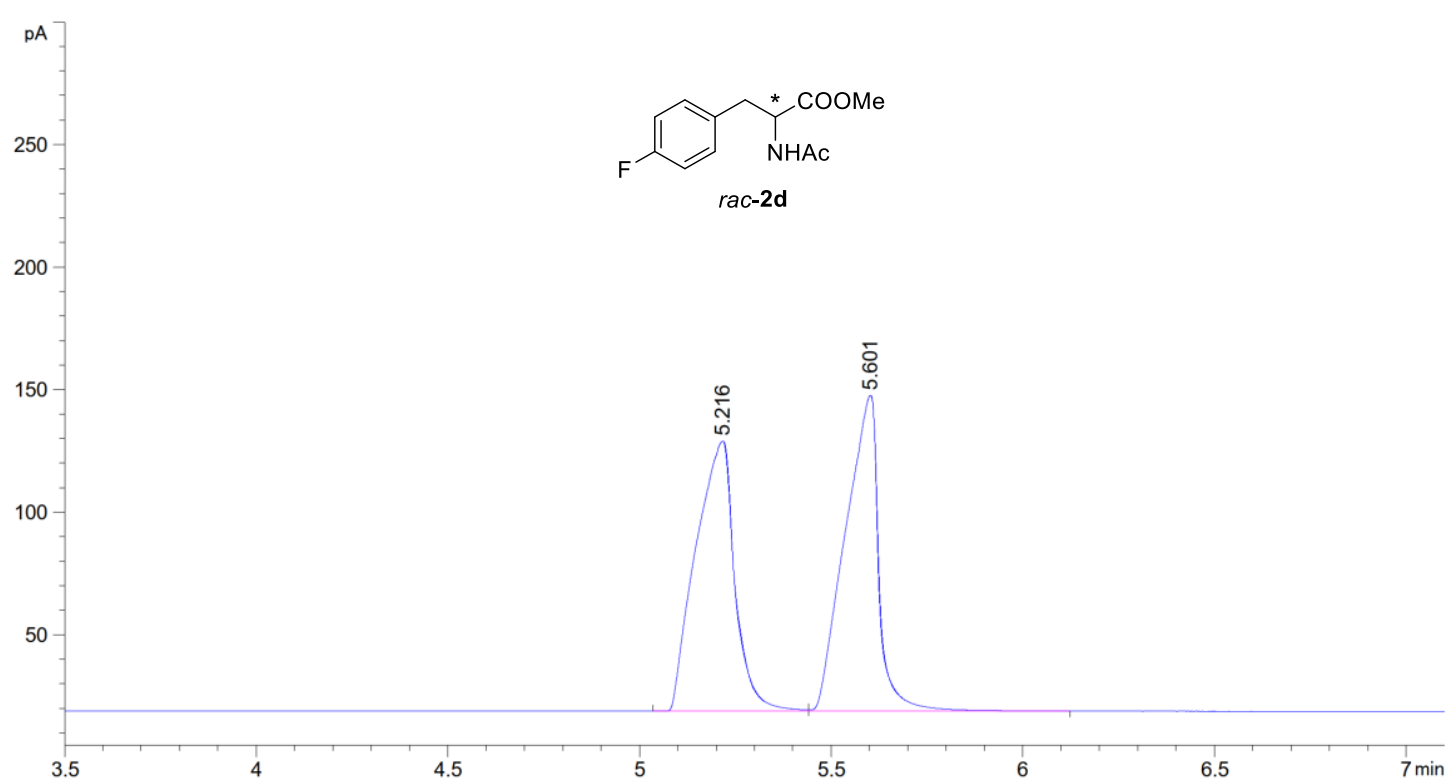

峰 保留时间 类型 峰宽 峰面积峰高峰面积

$\begin{array}{cccccc}\# & {[\mathrm{~min}]} & {[\mathrm{min}]} & {\left[\mathrm{pA} *_{\mathrm{s}}\right]} & {[\mathrm{pA}]} & \% \\ 1 & 5.216 \text { BV } & 0.0930 & 780.13898 & 110.18624 & 49.87475 \\ 2 & 5.601 \mathrm{VB} & 0.0846 & 784.05743 & 128.75928 & 50.12525\end{array}$

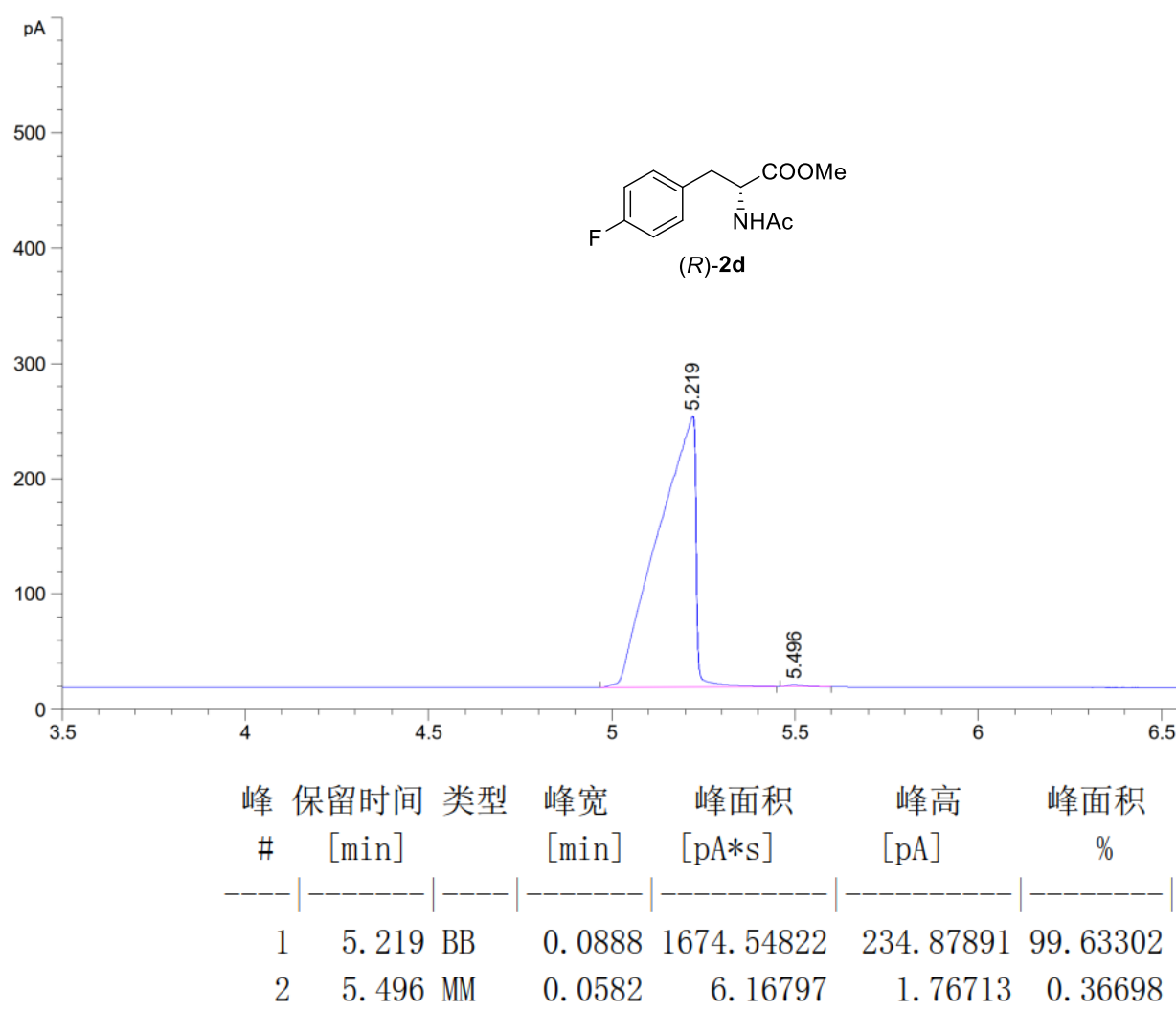



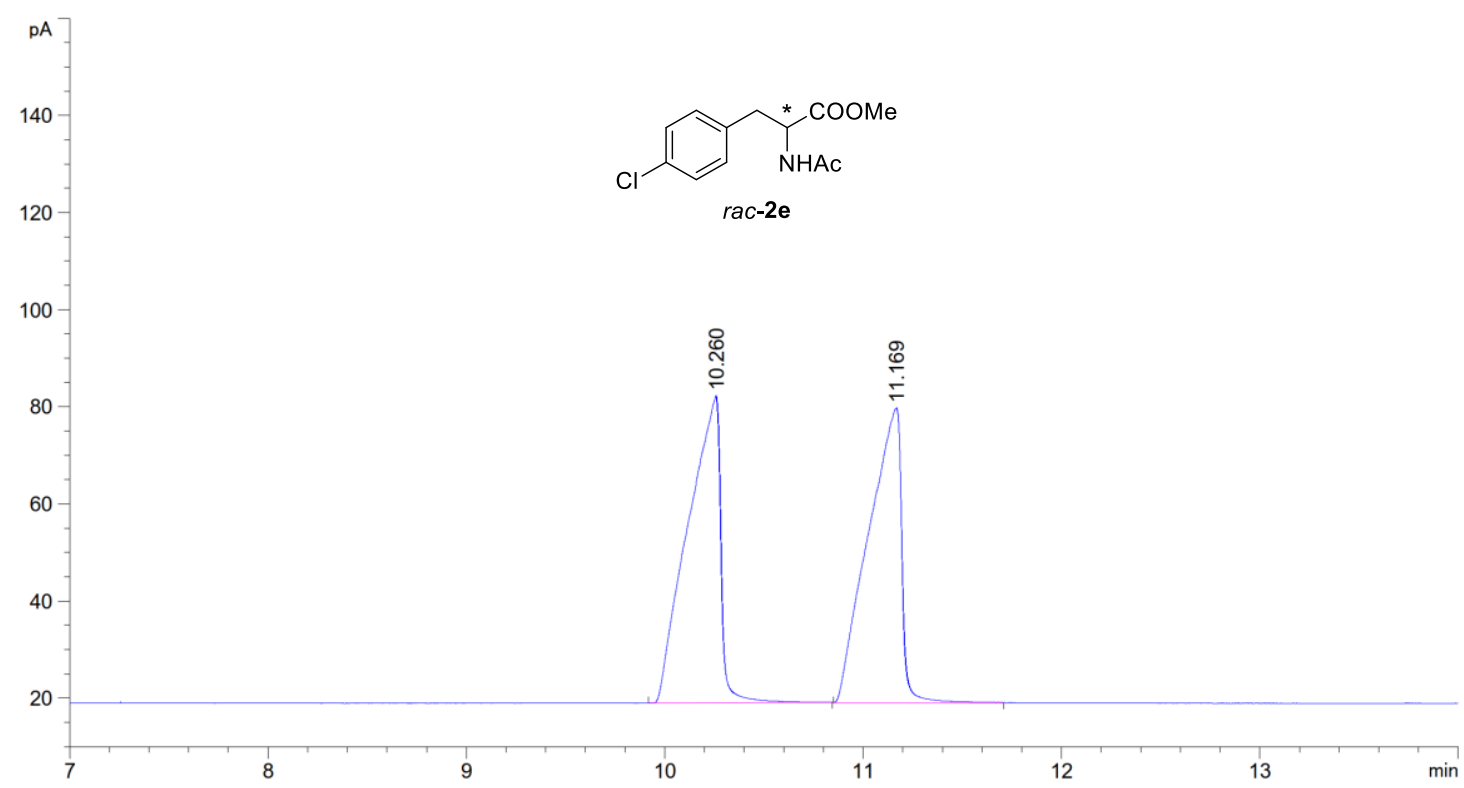

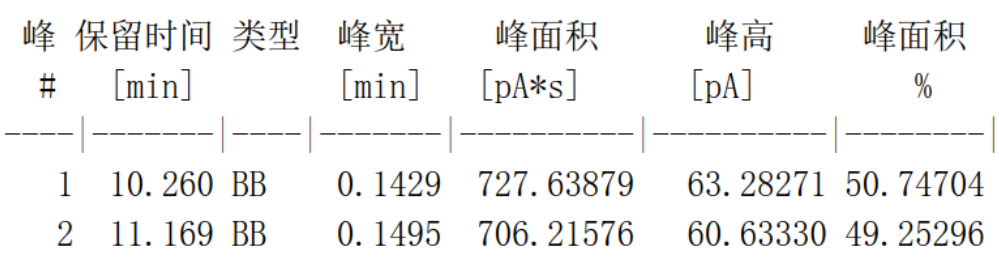

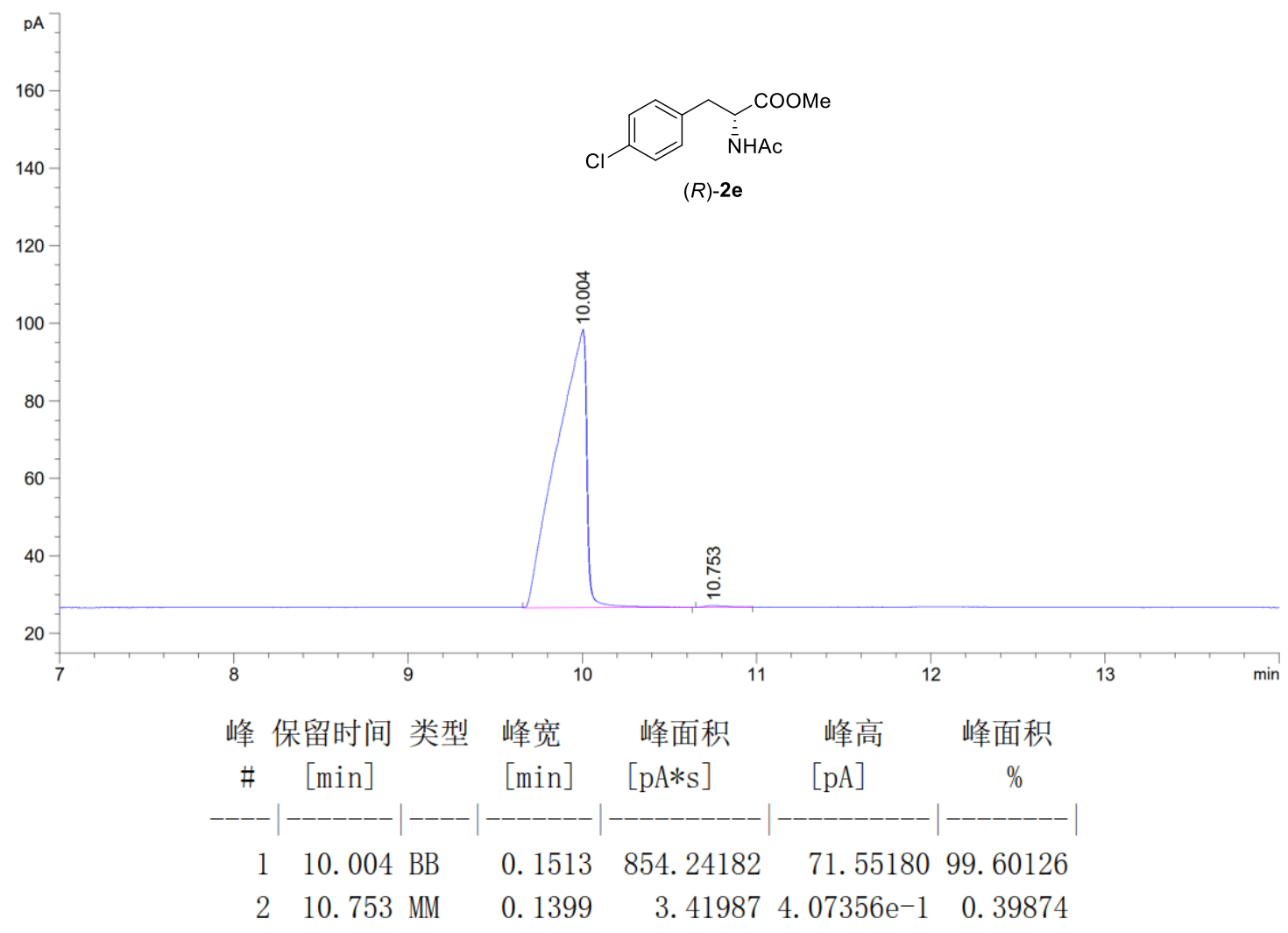




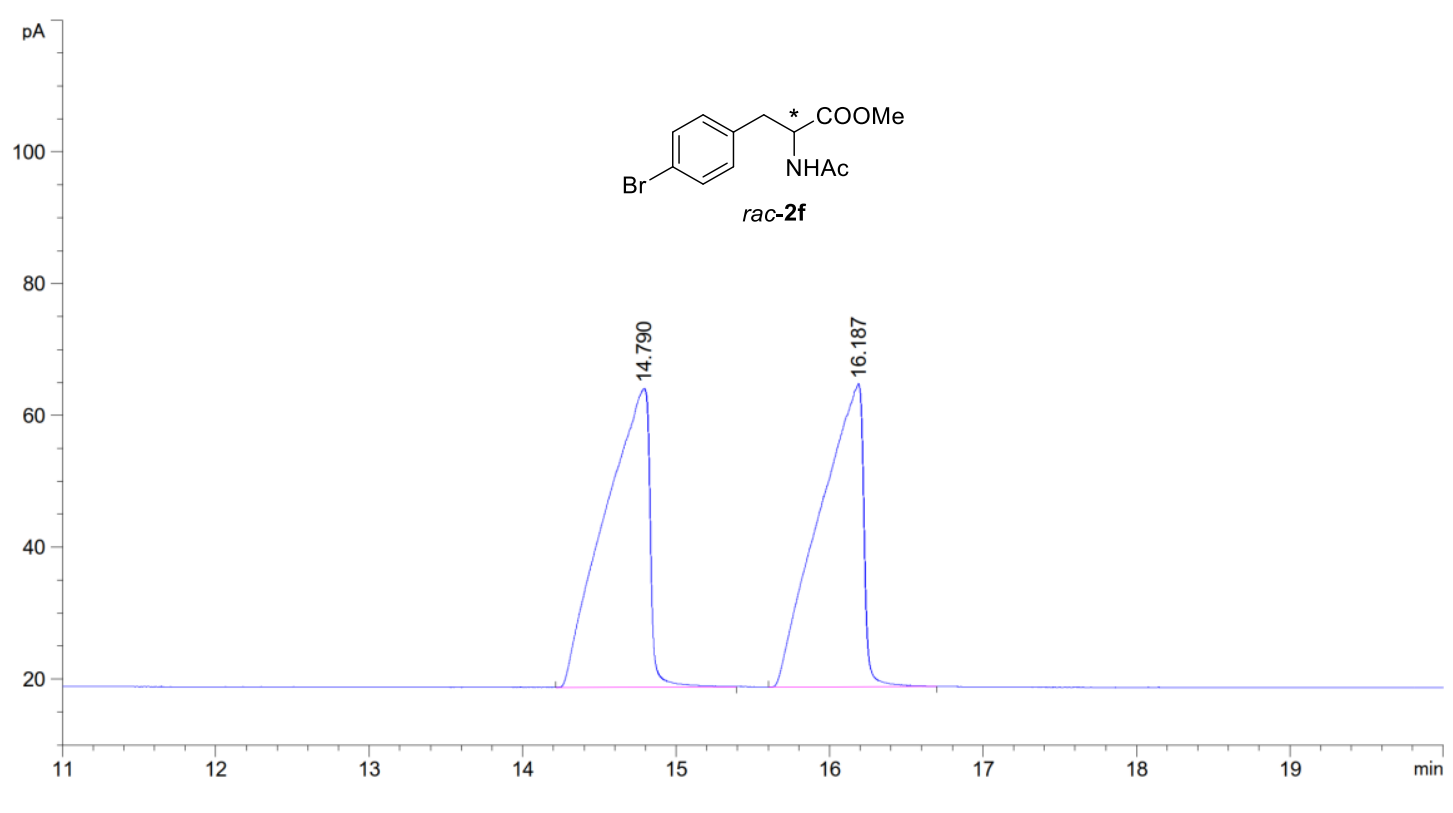

\begin{tabular}{|c|c|c|c|c|c|c|}
\hline $\begin{array}{r}\text { 峰 } \\
\#\end{array}$ & $\begin{array}{c}\text { 保留时间 } \\
\text { [min] }\end{array}$ & 类型 & $\begin{array}{l}\text { 峰宽 } \\
\text { [min] }\end{array}$ & $\begin{array}{c}\text { 峰面积 } \\
{\left[\mathrm{pA} *_{\mathrm{s}}\right]}\end{array}$ & $\begin{array}{c}\text { 峰高 } \\
{[\mathrm{pA}]}\end{array}$ & $\begin{array}{c}\text { 峰面积 } \\
\%\end{array}$ \\
\hline & & & 04 & 917. & 833 & 36 \\
\hline 2 & 16.18 & $\mathrm{BB}$ & 0.2462 & 913.64734 & 45.98726 & 9. 9056 \\
\hline
\end{tabular}

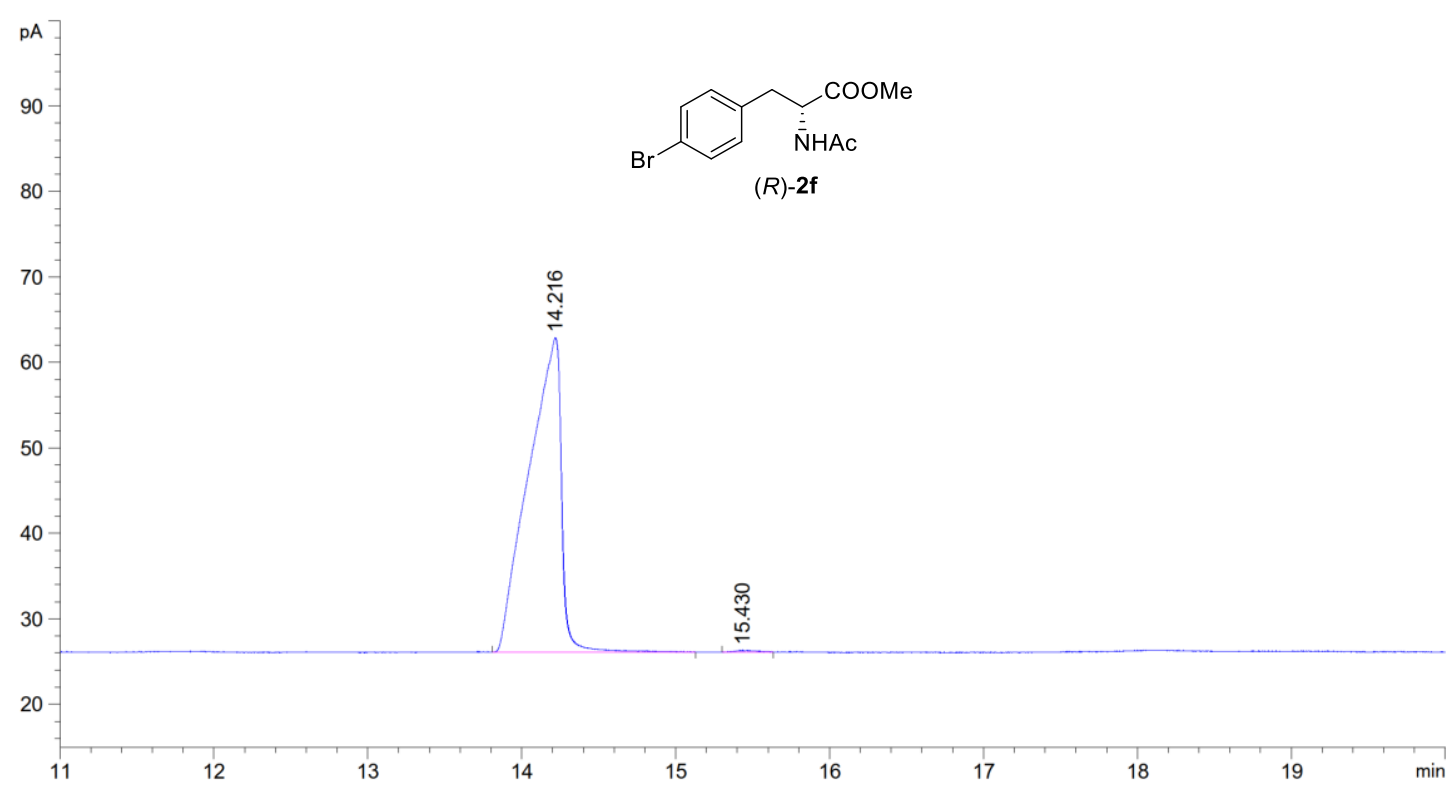

峰 保留时间 类型 峰宽 峰面积峰高峰面积

\begin{tabular}{cccccc}
$\#$ & {$[\mathrm{~min}]$} & {$[\mathrm{min}]$} & {$[\mathrm{pA} * \mathrm{~s}]$} & \multicolumn{1}{c}{$[\mathrm{pA}]$} & $\%$ \\
-1 & $14.216 \mathrm{BB}$ & 0.1914 & 545.69977 & 36.79535 & 99.62859 \\
2 & $15.430 \mathrm{MM}$ & 0.1593 & 2.03432 & $2.12815 \mathrm{e}^{-1}$ & 0.37141
\end{tabular}



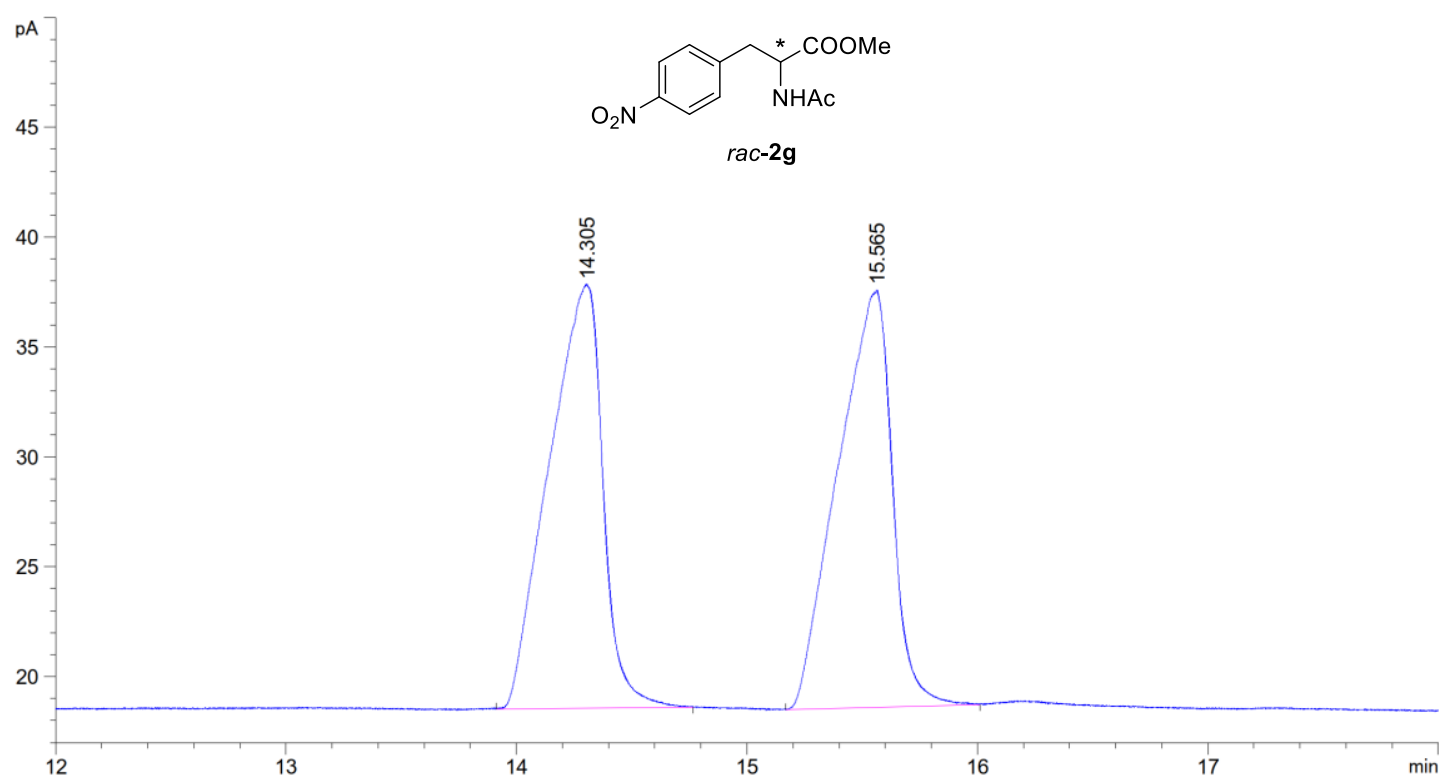

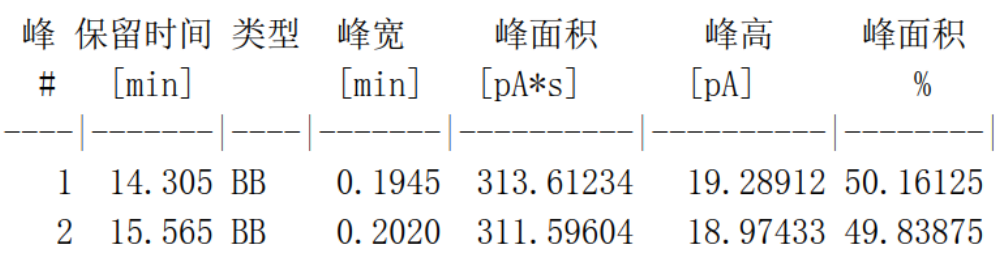

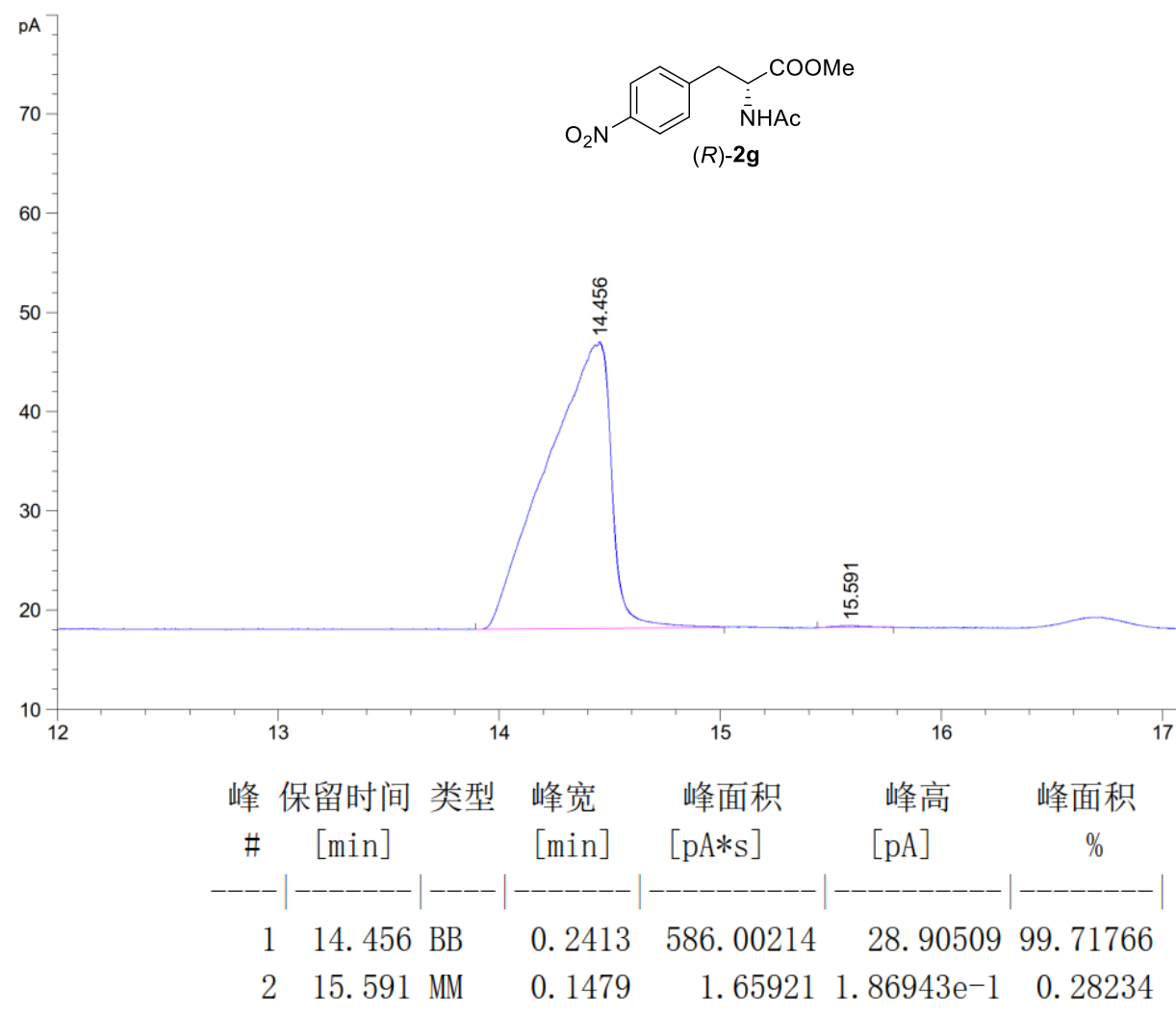




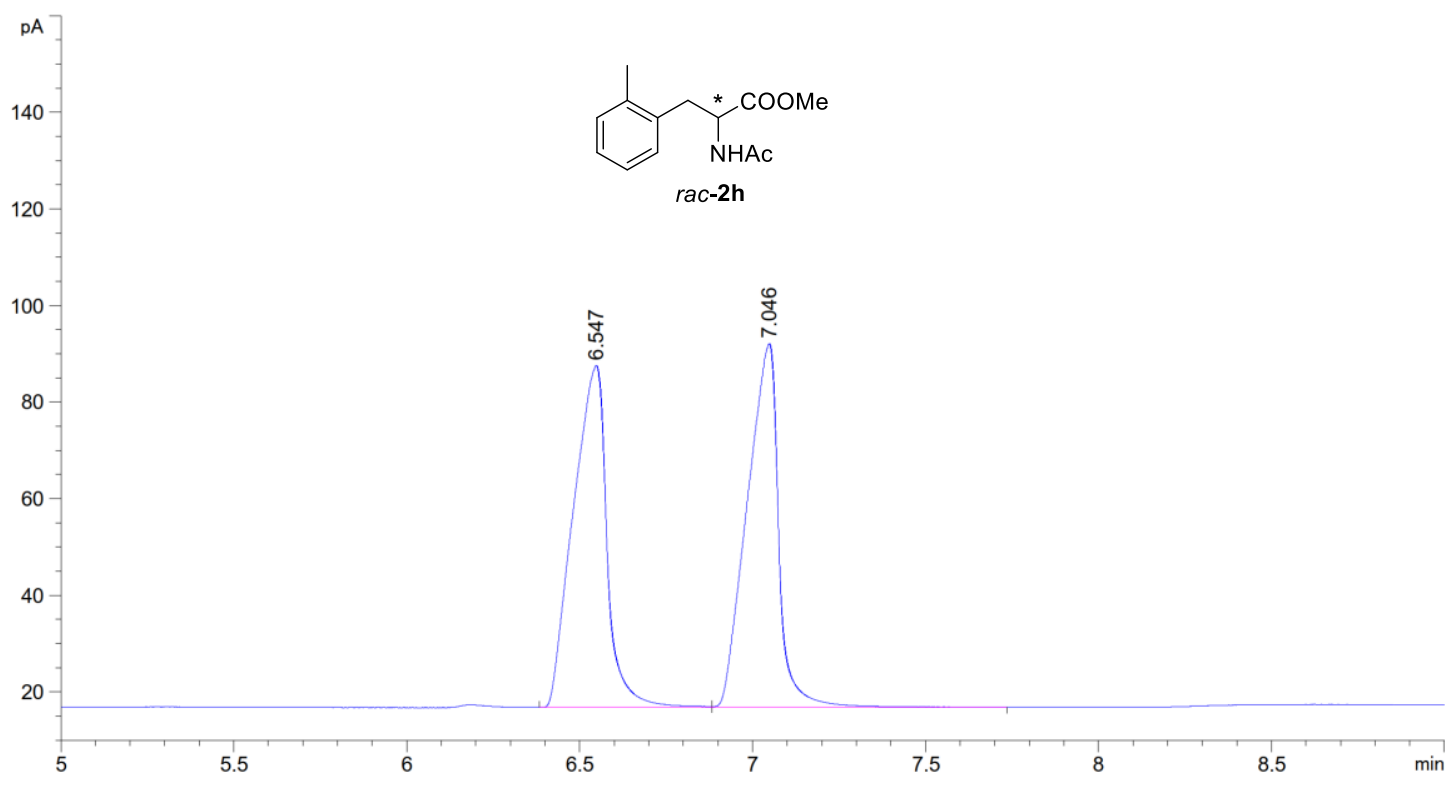

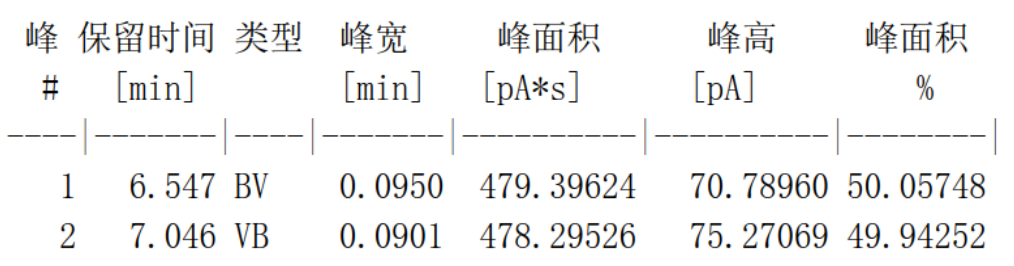

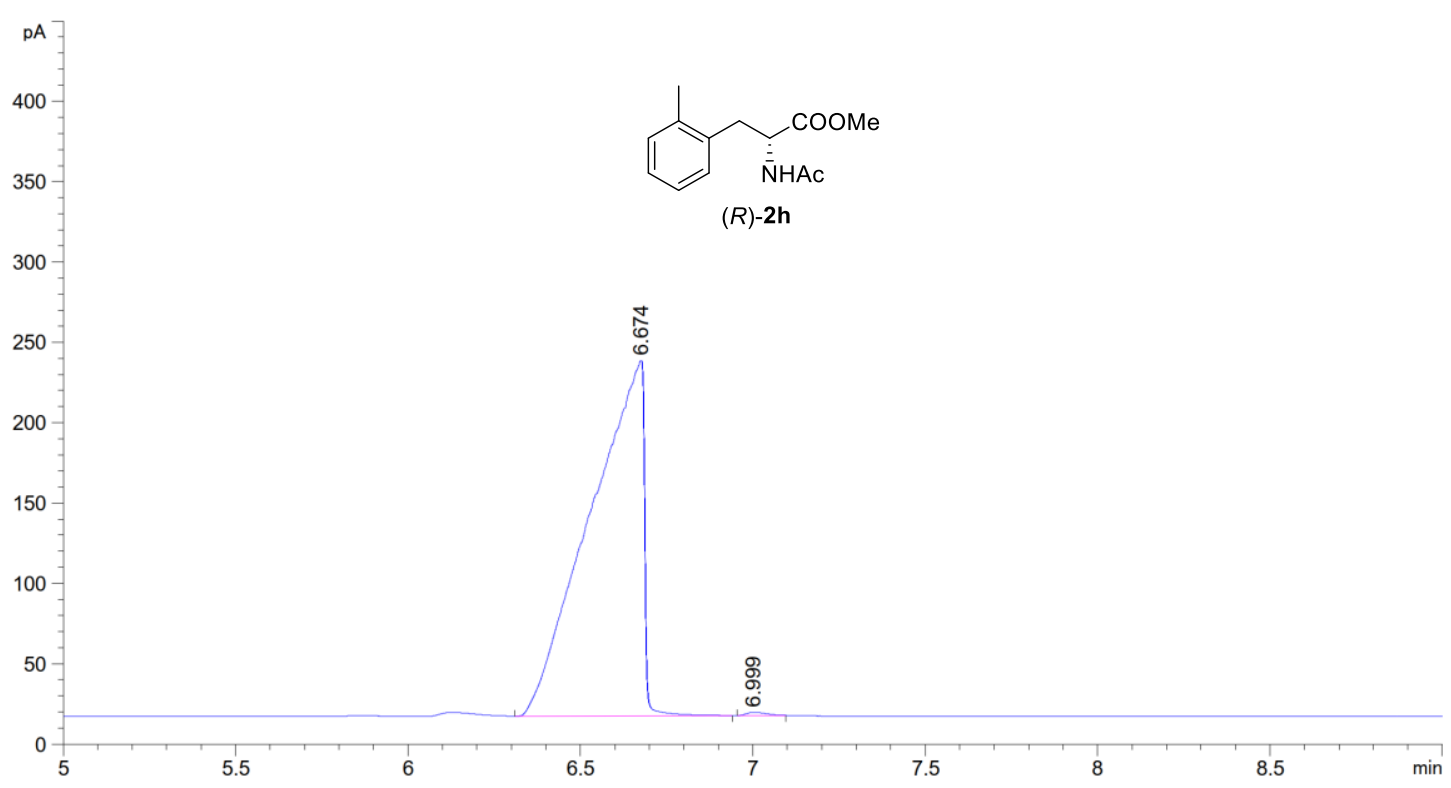

峰 保留时间 类型 峰宽 峰面积峰高 峰面积

\begin{tabular}{cccccc}
$\#$ & {$[\mathrm{~min}]$} & {$[\mathrm{min}]$} & {$[\mathrm{pA}$ s $]$} & \multicolumn{1}{c}{$[\mathrm{pA}]$} & $\%$ \\
\hline 1 & 6.674 BB & 0.1316 & 2400.15967 & 220.79480 & 99.68669 \\
2 & $6.999 \mathrm{MM}$ & 0.0655 & 7.54352 & 1.91810 & 0.31331
\end{tabular}




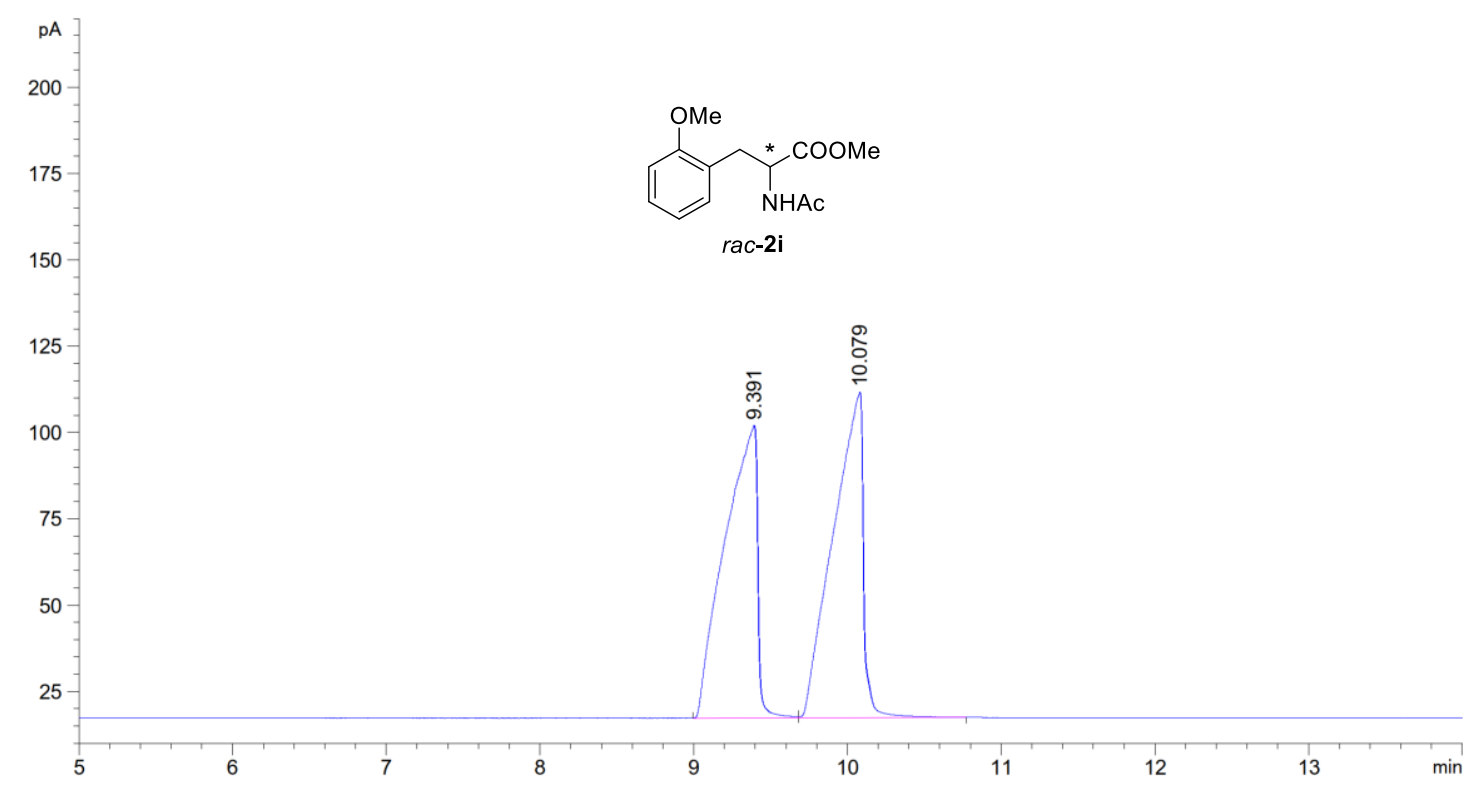

峰 保留时间 类型 峰宽 峰面积 峰高 峰面积

\begin{tabular}{crcccc}
$\#$ & {$[\mathrm{~min}]$} & {$[\mathrm{min}]$} & {$[\mathrm{pA} * \mathrm{~s}]$} & {$[\mathrm{pA}]$} & $\%$ \\
\hline 1 & 9.391 BV & 0.1817 & 1250.81128 & 84.77244 & 49.35052 \\
2 & 10.079 VB & 0.1668 & 1283.73425 & 94.29718 & 50.64948
\end{tabular}

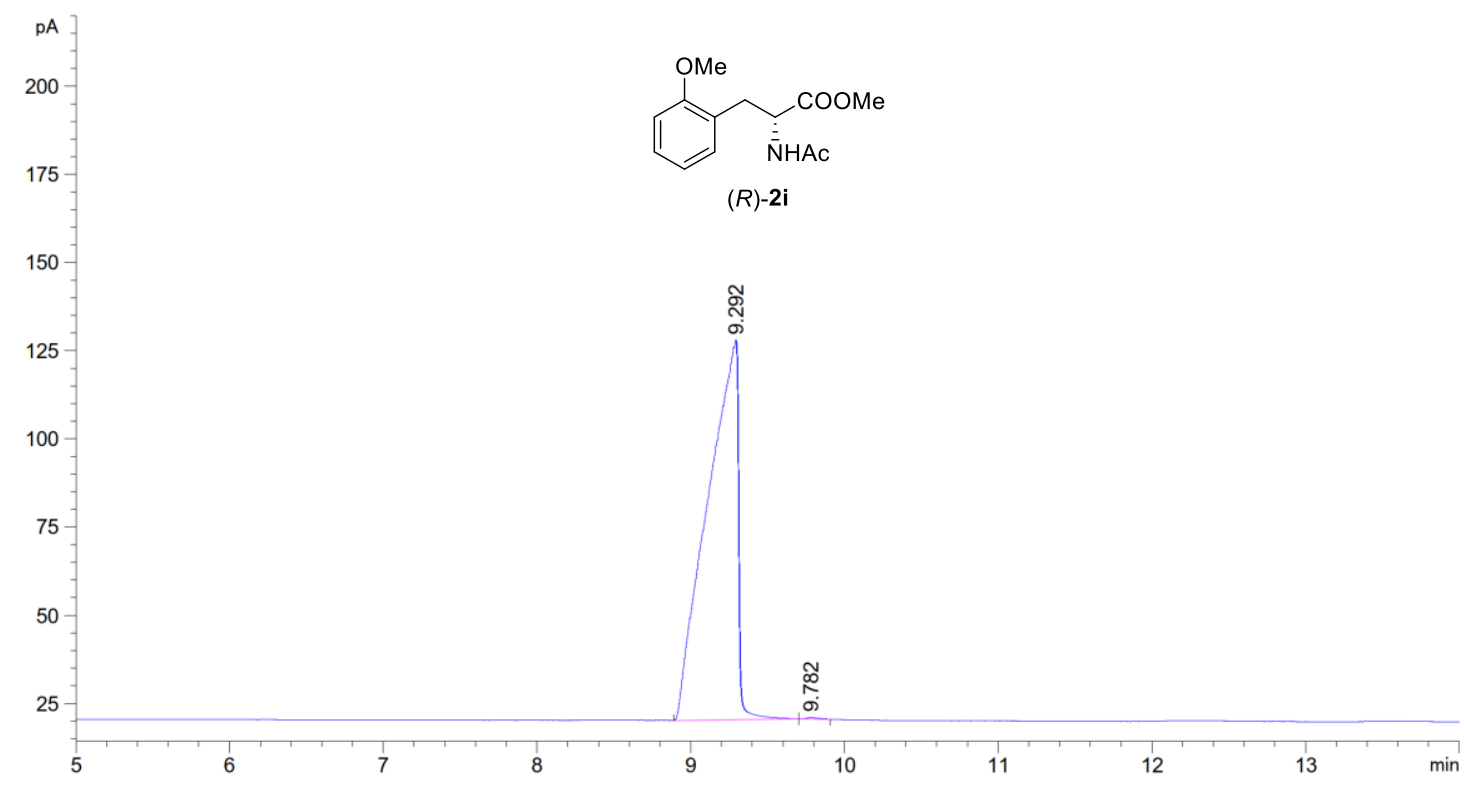

\begin{tabular}{|c|c|c|c|c|c|c|}
\hline $\begin{array}{l}\text { 峰 } \\
\text { \# }\end{array}$ & $\begin{array}{c}\text { 保留时间 } \\
\text { [min] }\end{array}$ & 类型 & $\begin{array}{l}\text { 峰宽 } \\
\text { [min] }\end{array}$ & $\begin{array}{c}\text { 峰面积 } \\
{[\mathrm{pA} * \mathrm{~s}]}\end{array}$ & $\begin{array}{l}\text { 峰高 } \\
\text { [pA] }\end{array}$ & $\begin{array}{c}\text { 峰面积 } \\
\%\end{array}$ \\
\hline 1 & 9.29 & & 0.1684 & 1486. 78406 & 107. 57627 & 99.83242 \\
\hline 2 & 9.782 & MM & 0.0959 & 2. 49578 & 4. $33805 \mathrm{e}^{-1}$ & 0.16758 \\
\hline
\end{tabular}




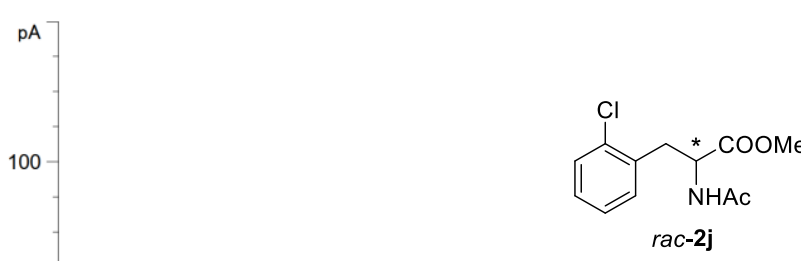

80

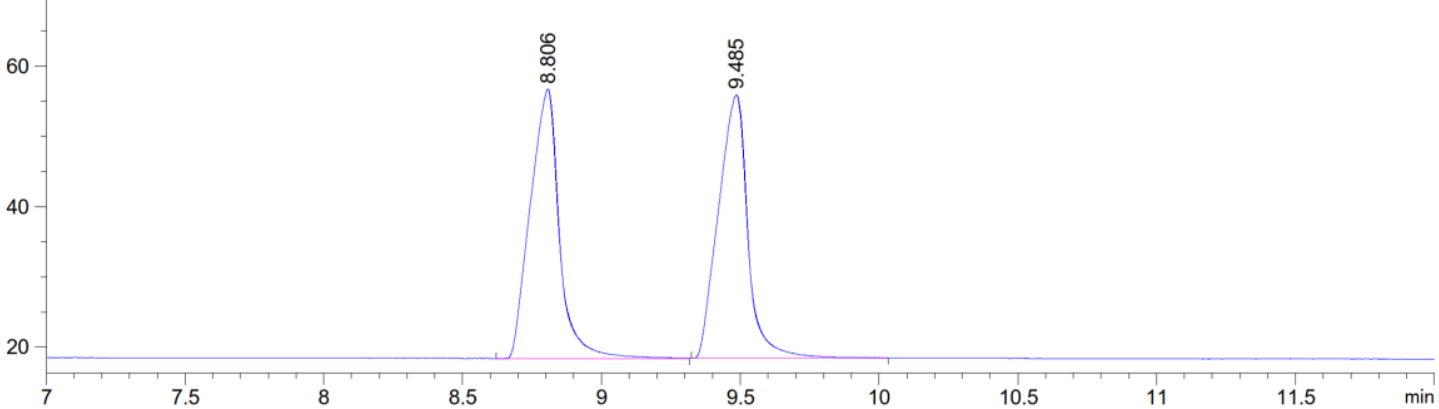

峰 保留时间 类型 峰宽 峰面积 峰高 峰面积

\begin{tabular}{cccccc}
$\#$ & {$[\mathrm{~min}]$} & {$[\mathrm{min}]$} & {$[\mathrm{pA} * \mathrm{~s}]$} & {$[\mathrm{pA}]$} & $\%$ \\
\hline 1 & $8.806 \mathrm{BB}$ & 0.0995 & 279.46207 & 38.33184 & 50.28126 \\
2 & $9.485 \mathrm{BB}$ & 0.0989 & 276.33563 & 37.44623 & 49.71874
\end{tabular}
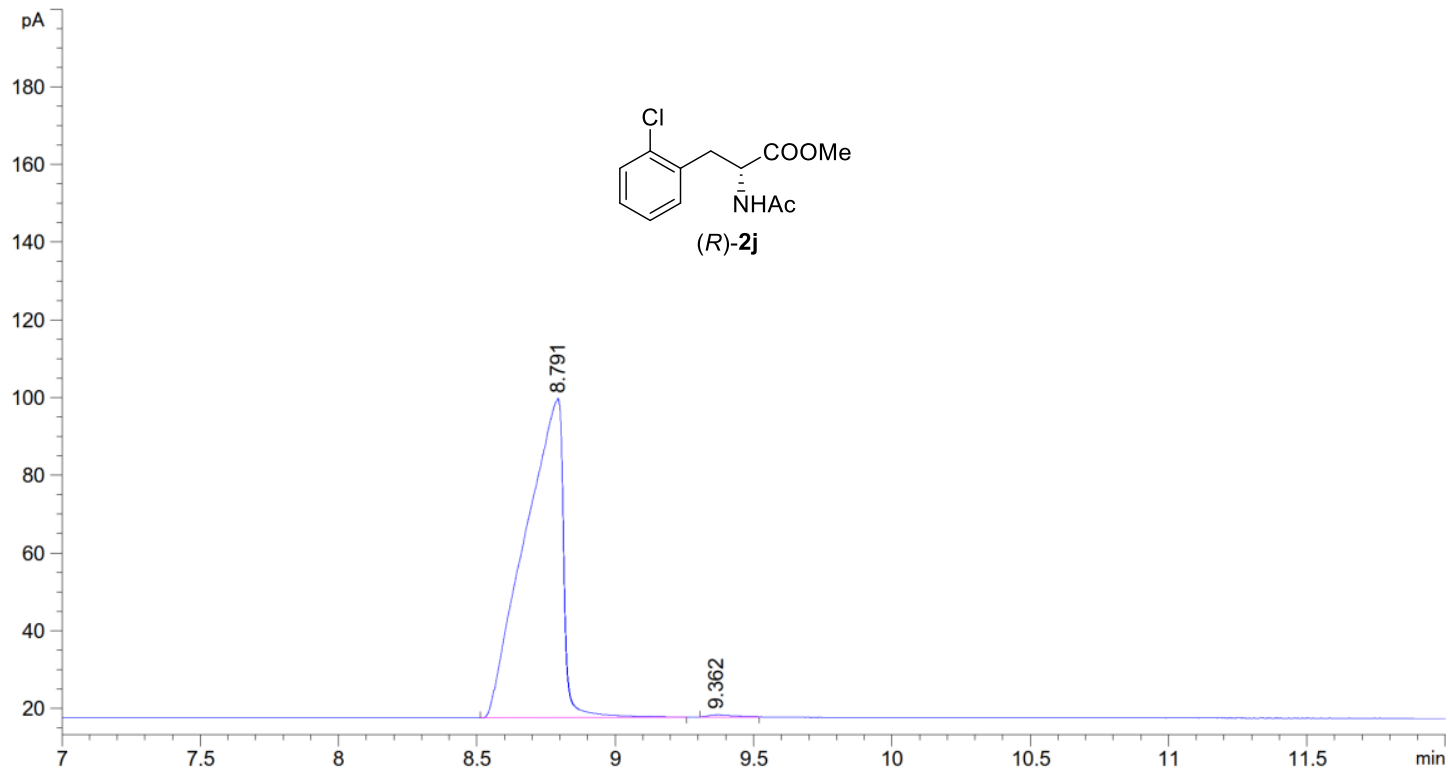

峰 保留时间 类型 峰宽 峰面积峰高峰面积

$\begin{array}{cccccc}\# & {[\mathrm{~min}]} & {[\mathrm{min}]} & {[\mathrm{pA} * \mathrm{~s}]} & {[\mathrm{pA}]} & \% \\ -1 & 8.791 \mathrm{BB} & 0.1194 & 789.50903 & 82.08967 & 99.58546 \\ 2 & 9.362 \mathrm{MM} & 0.1018 & 3.28644 & 5.38177 \mathrm{e}^{-1} & 0.41454\end{array}$




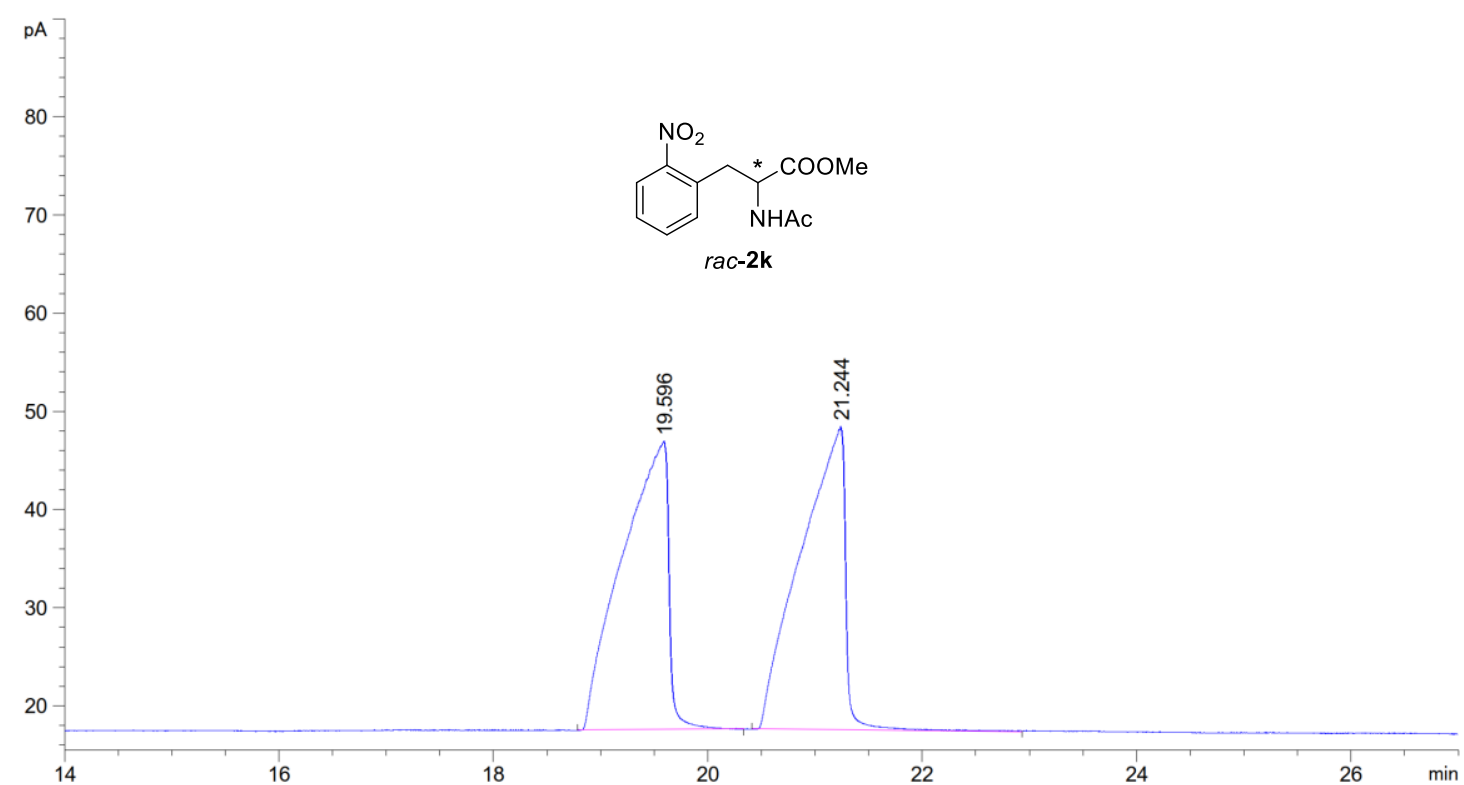

\begin{tabular}{|c|c|c|c|c|c|c|}
\hline $\begin{array}{c}\text { 峰 } \\
\text { \# }\end{array}$ & $\begin{array}{c}\text { 保留时间 } \\
\text { [min] }\end{array}$ & 类型 & $\begin{array}{l}\text { 峰宽 } \\
{[\mathrm{min}]}\end{array}$ & $\begin{array}{c}\text { 峰面积 } \\
{\left[\mathrm{pA} *_{\mathrm{S}}\right]}\end{array}$ & $\begin{array}{l}\text { 峰高 } \\
{[\mathrm{pA}]}\end{array}$ & $\begin{array}{c}\text { 峰面积 } \\
\%\end{array}$ \\
\hline & & & 0. & 878 & 29 & . \\
\hline & 21.2 & & 0.4802 & 890.18488 & 30.89749 & 50.3275 \\
\hline
\end{tabular}

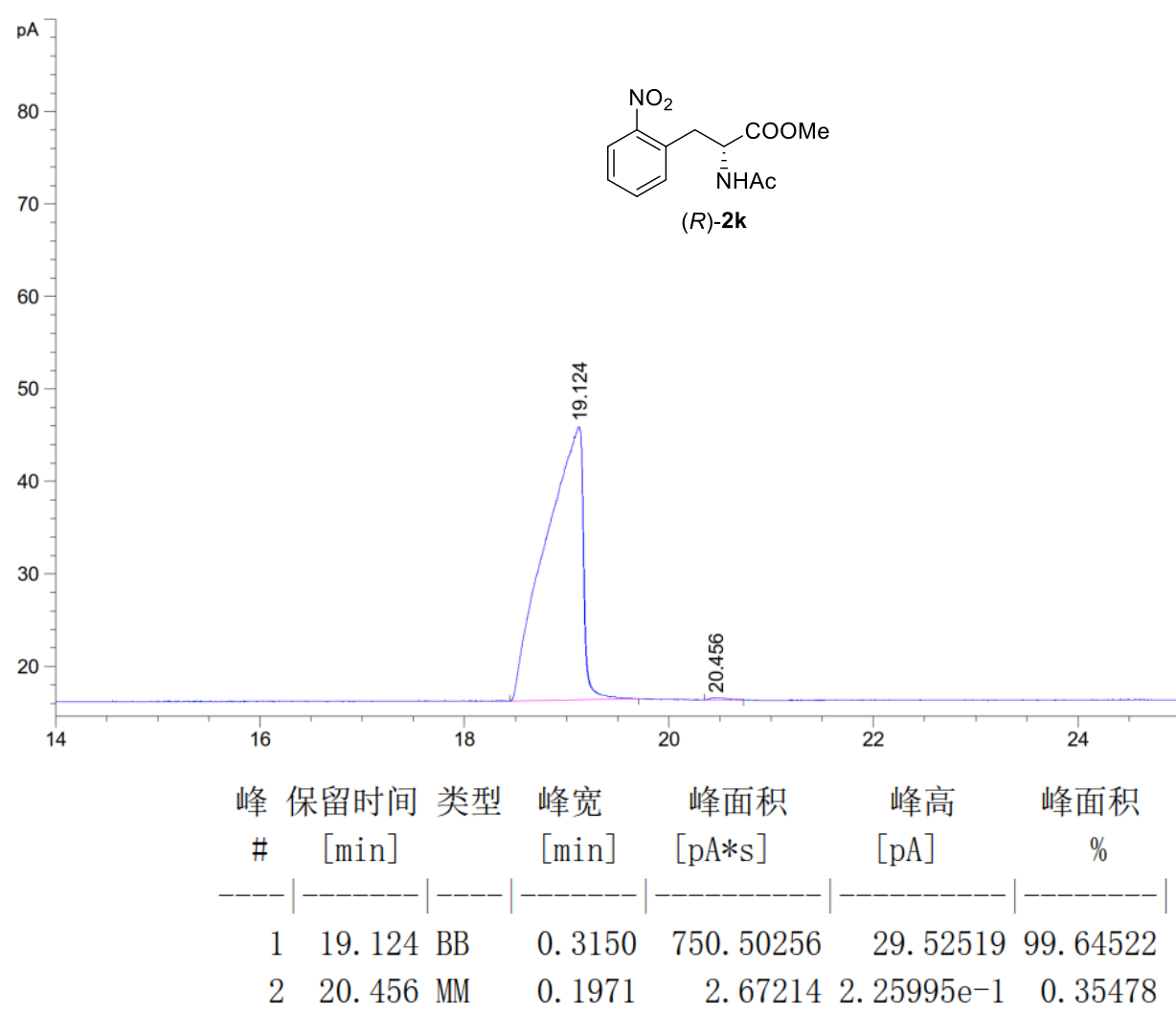



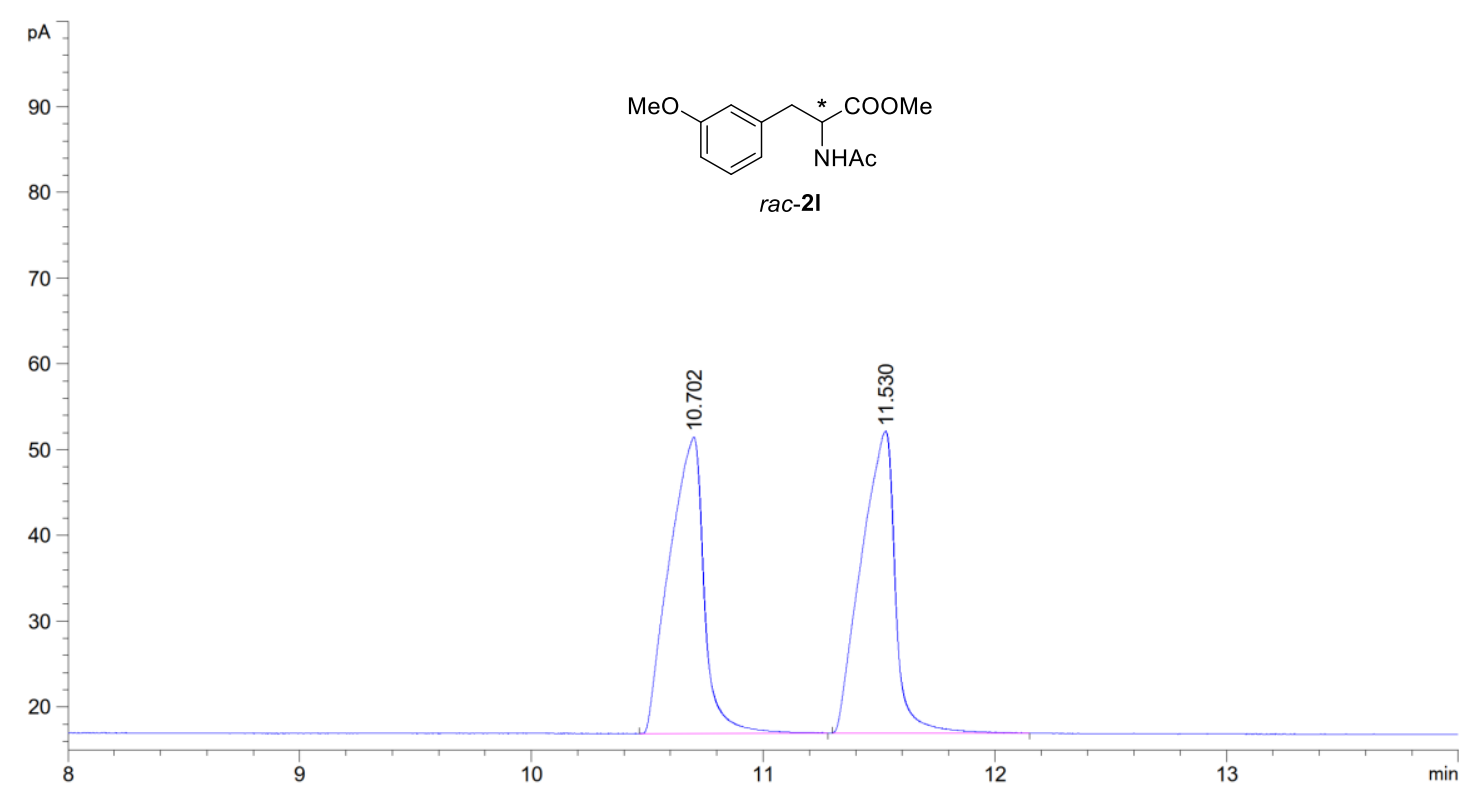

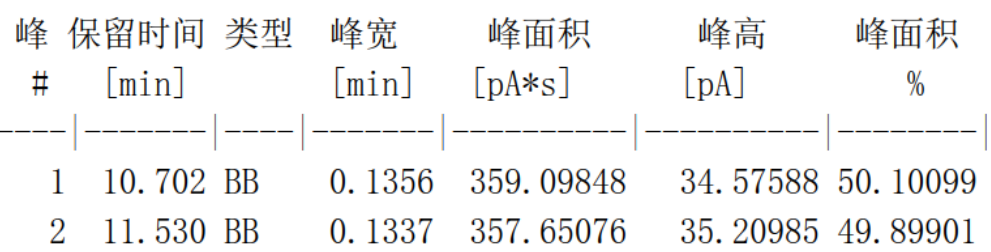

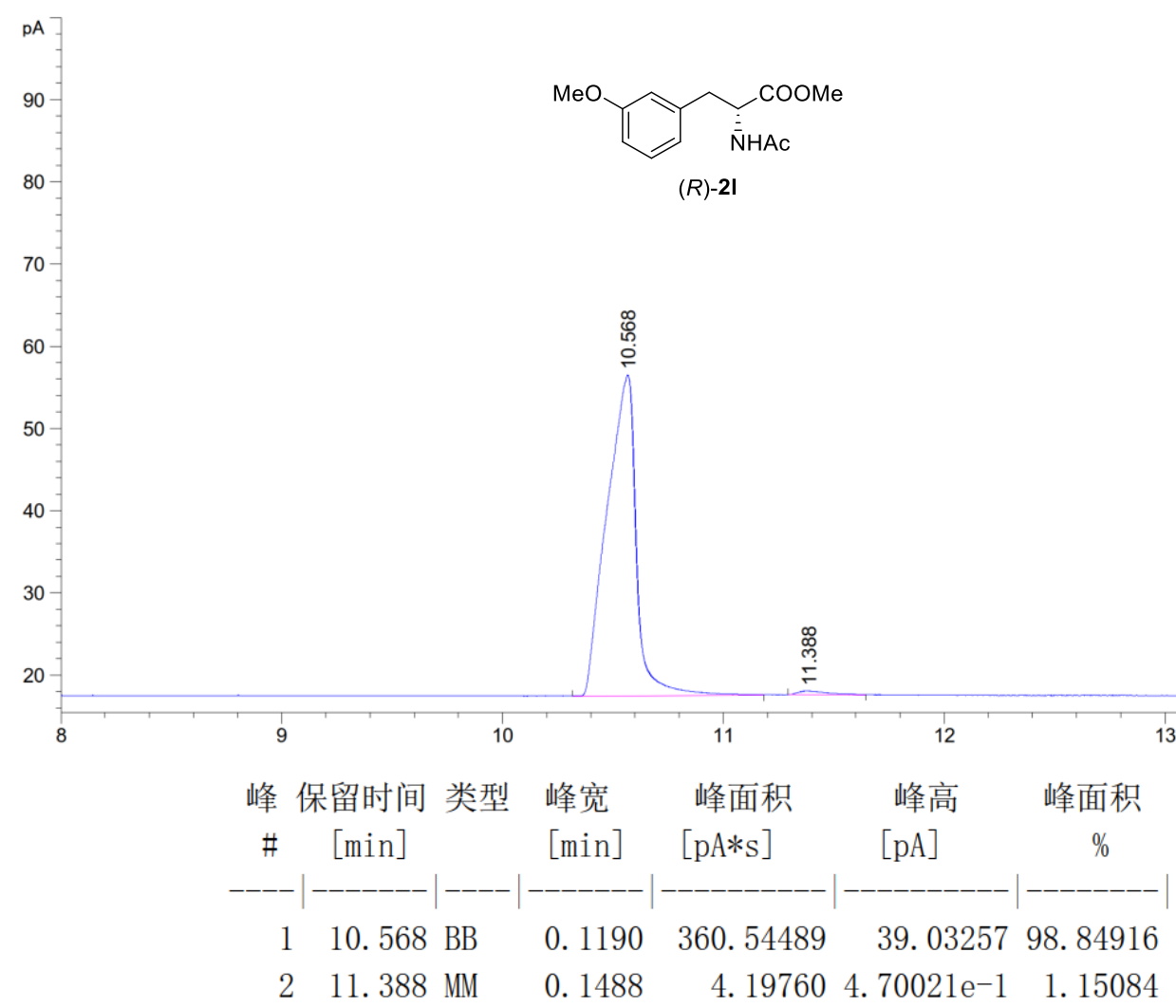




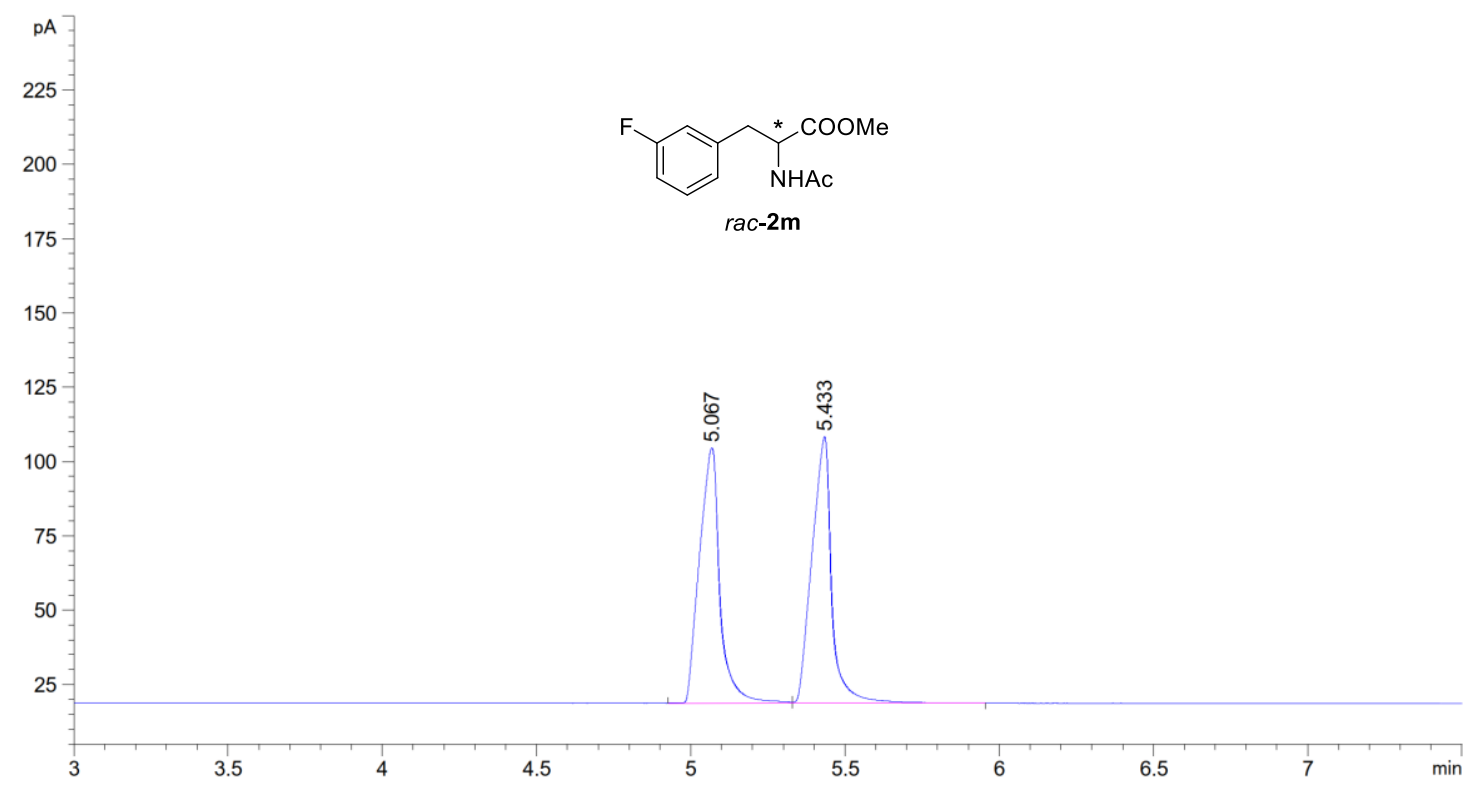

峰 保留时间 类型 峰宽峰面积峰高峰面积

$\begin{array}{cccccc}\# & {[\mathrm{~min}]} & {[\mathrm{min}]} & {[\mathrm{pA} * \mathrm{~s}]} & {[\mathrm{pA}]} & \% \\ 1 & 5.067 \text { BV } & 0.0747 & 396.46988 & 85.87380 & 49.88262 \\ 2 & 5.433 \mathrm{VB} & 0.0655 & 398.33578 & 89.56658 & 50.11738\end{array}$

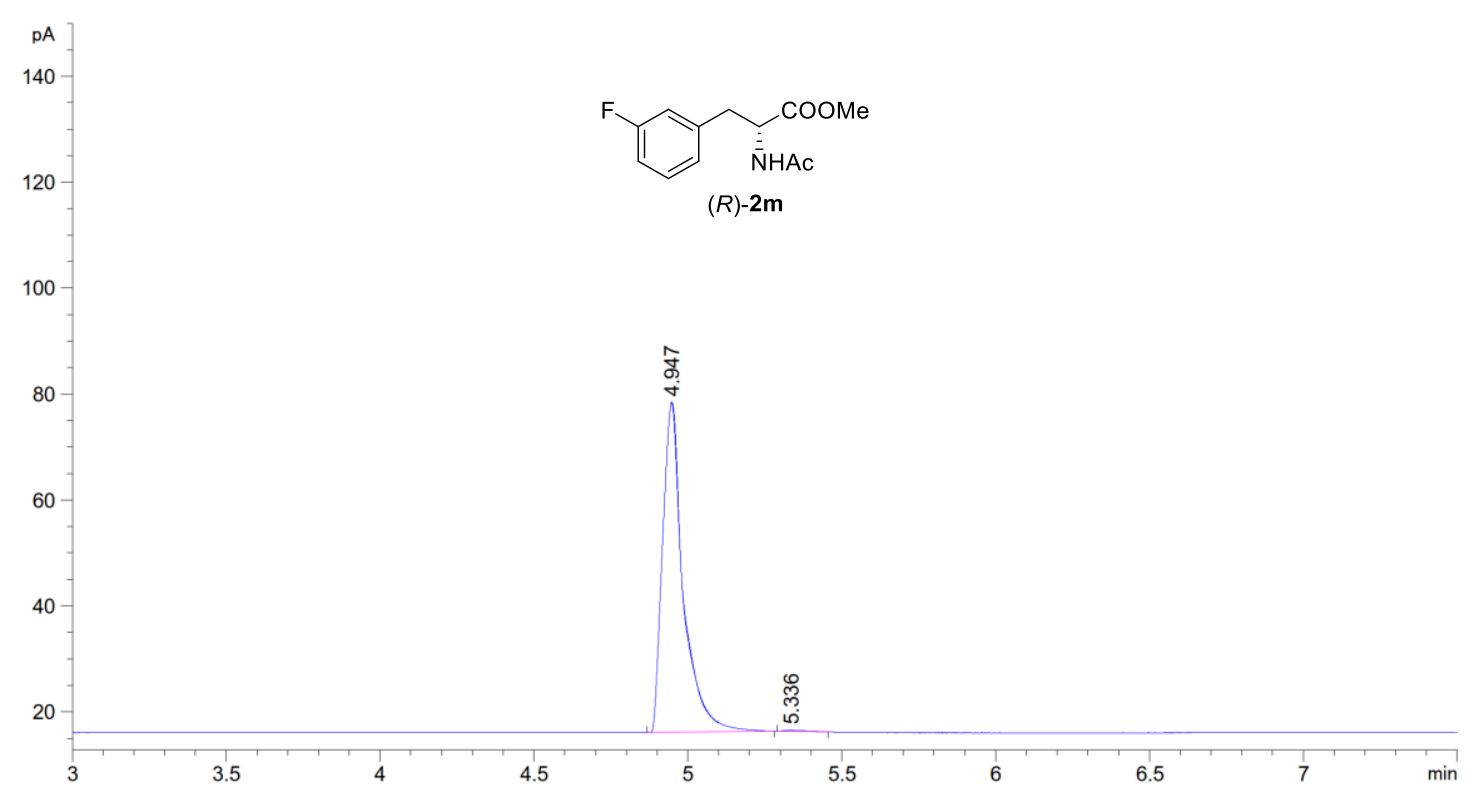

峰 保留时间 类型 峰宽 峰面积 峰高 峰面积

\begin{tabular}{ccccrc}
$\#$ & {$[\mathrm{~min}]$} & {$[\mathrm{min}]$} & {$[\mathrm{pA} * \mathrm{~s}]$} & \multicolumn{1}{c}{$[\mathrm{pA}]$} & $\%$ \\
\hline 1 & 4.947 BB & 0.0719 & 290.28851 & 62.35990 & 99.62298 \\
2 & 5.336 MM & 0.0752 & 1.09859 & $2.43331 \mathrm{e}^{-1}$ & 0.37702
\end{tabular}




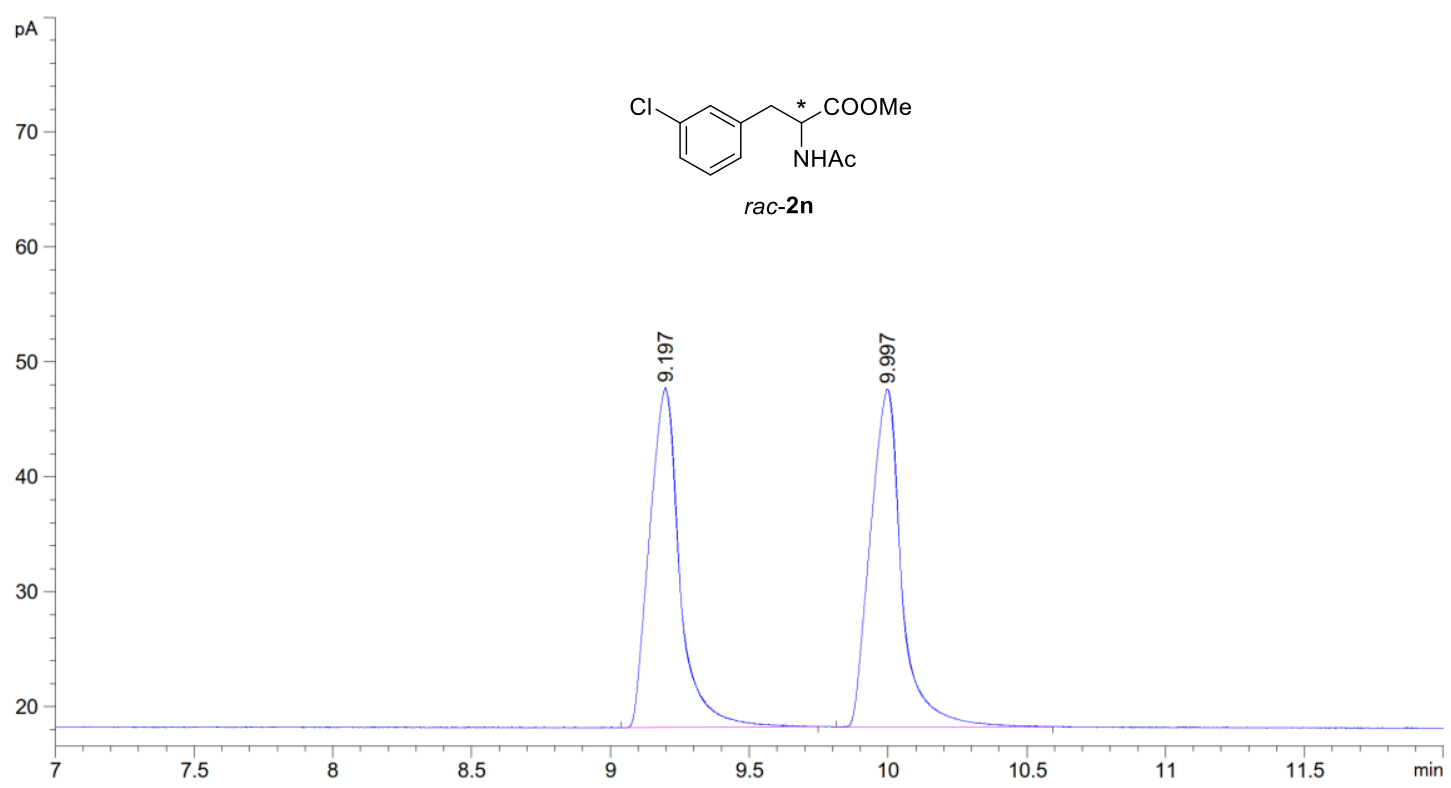

峰 保留时间 类型 峰宽 峰面积峰高峰面积

\begin{tabular}{cccccc}
$\#$ & {$[\mathrm{~min}]$} & {$[\mathrm{min}]$} & {$[\mathrm{pA}$ s $]$} & {$[\mathrm{pA}]$} & $\%$ \\
\hline 1 & $9.197 \mathrm{BB}$ & 0.1078 & 224.54764 & 29.56320 & 49.30405 \\
2 & $9.997 \mathrm{BB}$ & 0.1092 & 230.88683 & 29.41247 & 50.69595
\end{tabular}

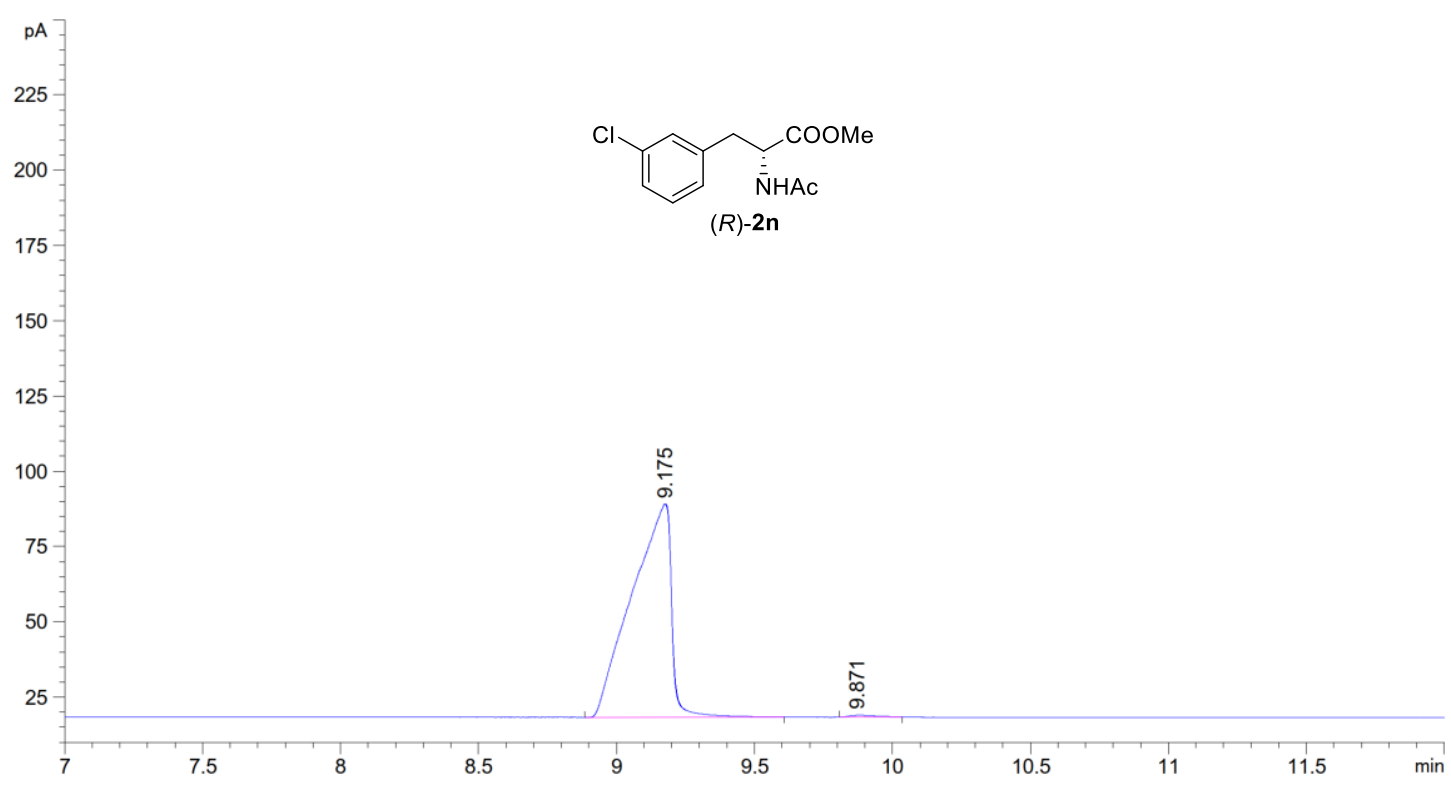

峰 保留时间 类型 峰宽 峰面积 峰高 峰面积

$\begin{array}{cccccc}\# & {[\mathrm{~min}]} & {[\mathrm{min}]} & {[\mathrm{pA} * \mathrm{~s}]} & {[\mathrm{pA}]} & \% \\ -1 & 9.175 \mathrm{BB} & 0.1249 & 704.92419 & 70.82798 & 99.40844 \\ 2 & 9.871 \mathrm{MM} & 0.1106 & 4.19488 & 6.32274 \mathrm{e}^{-1} & 0.59156\end{array}$



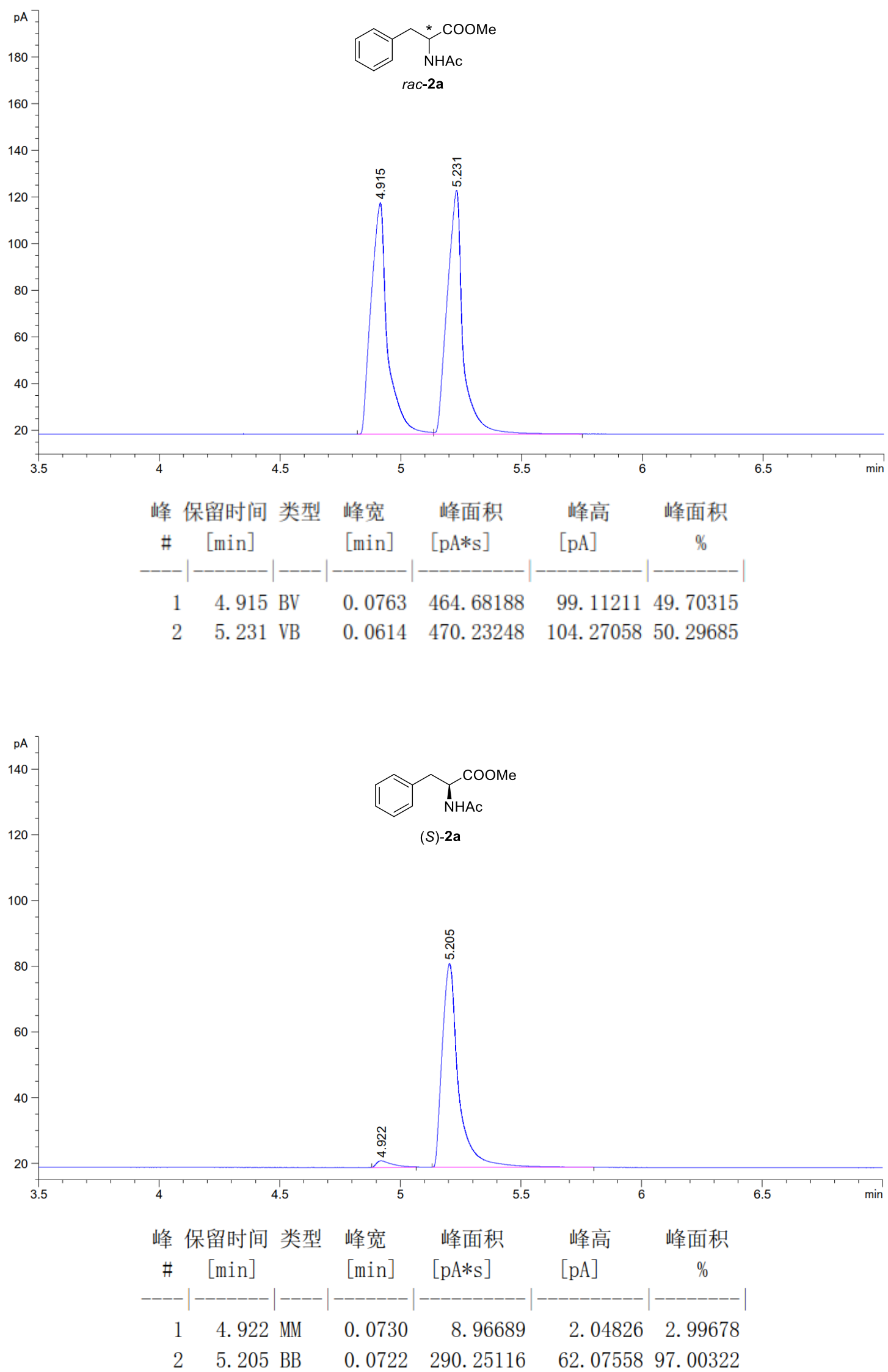


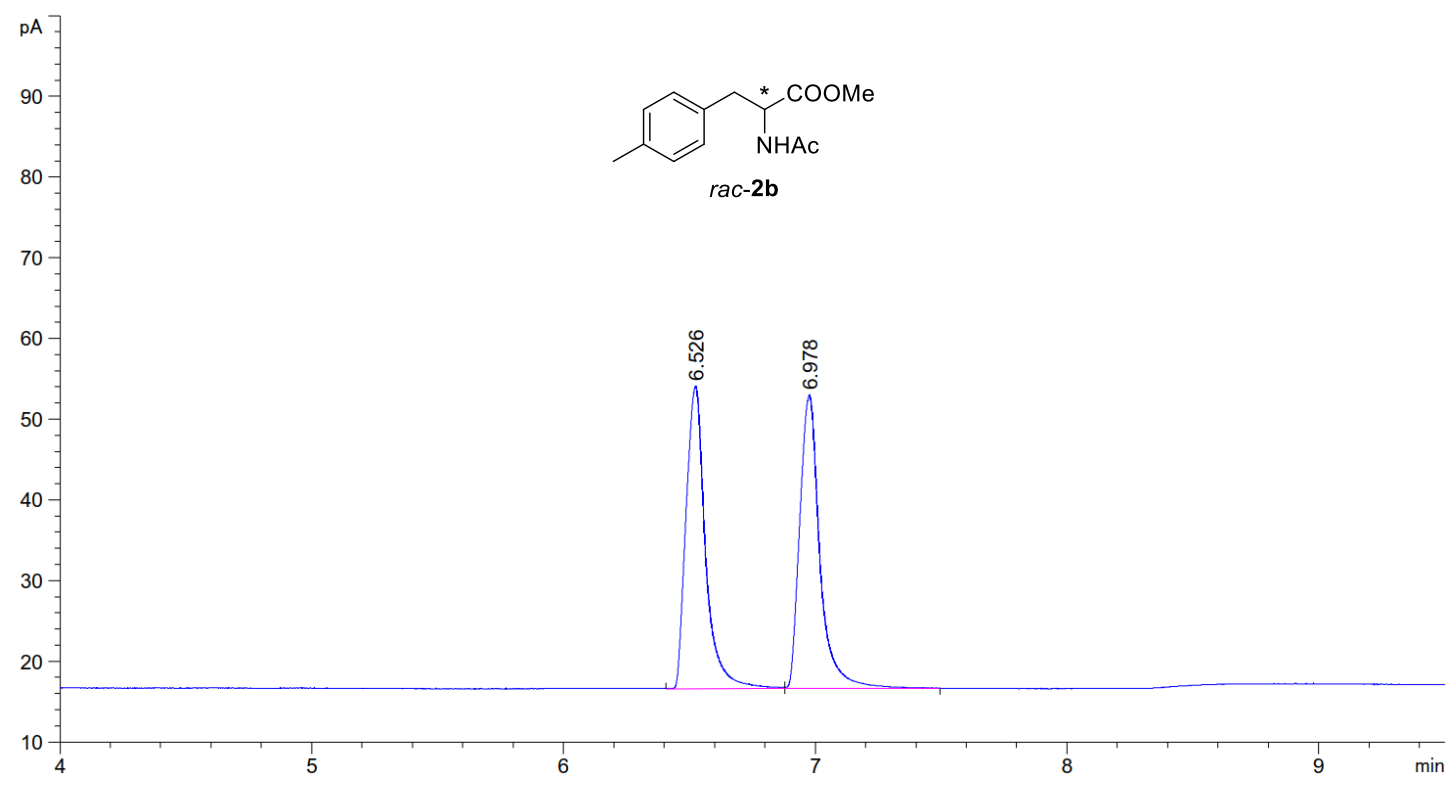

峰保留时间类型 峰宽 峰面积峰高峰面积

$\begin{array}{cccccc}\# & {[\mathrm{~min}]} & {[\mathrm{min}]} & {[\mathrm{pA} * \mathrm{~s}]} & {[\mathrm{pA}]} & \% \\ 1 & 6.526 \text { BV } & 0.0835 & 201.27539 & 37.47362 & 49.92747 \\ 2 & 6.978 \text { VB } & 0.0848 & 201.86021 & 36.32772 & 50.07253\end{array}$

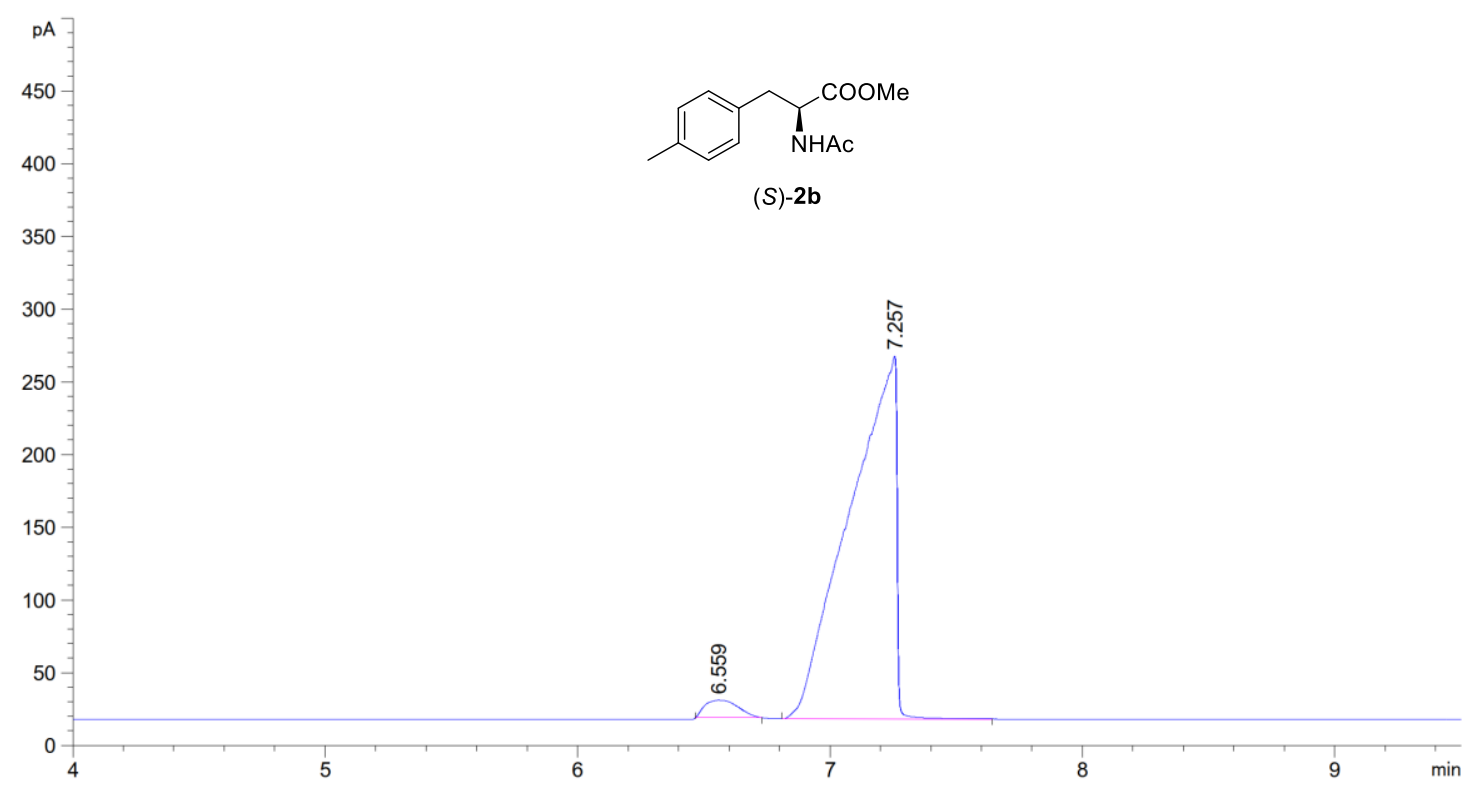

\begin{tabular}{|c|c|c|c|c|c|c|}
\hline $\begin{array}{c}\text { 峰 } \\
\#\end{array}$ & $\begin{array}{c}\text { 呆留时间 } \\
\text { [min] }\end{array}$ & 类型 & $\begin{array}{l}\text { 峰宽 } \\
\text { [min] }\end{array}$ & $\begin{array}{c}\text { 峰面积 } \\
{\left[\mathrm{pA} *_{\mathrm{s}}\right]}\end{array}$ & $\begin{array}{l}\text { 峰高 } \\
{[\mathrm{pA}]}\end{array}$ & $\begin{array}{c}\text { 峰面积 } \\
\%\end{array}$ \\
\hline 1 & 6.55 & & 0.1477 & 104. 45473 & 11. 78547 & 3. 13456 \\
\hline 2 & 7. 257 & r & 0.2152 & 3227.90771 & 250.04977 & 96.86544 \\
\hline
\end{tabular}



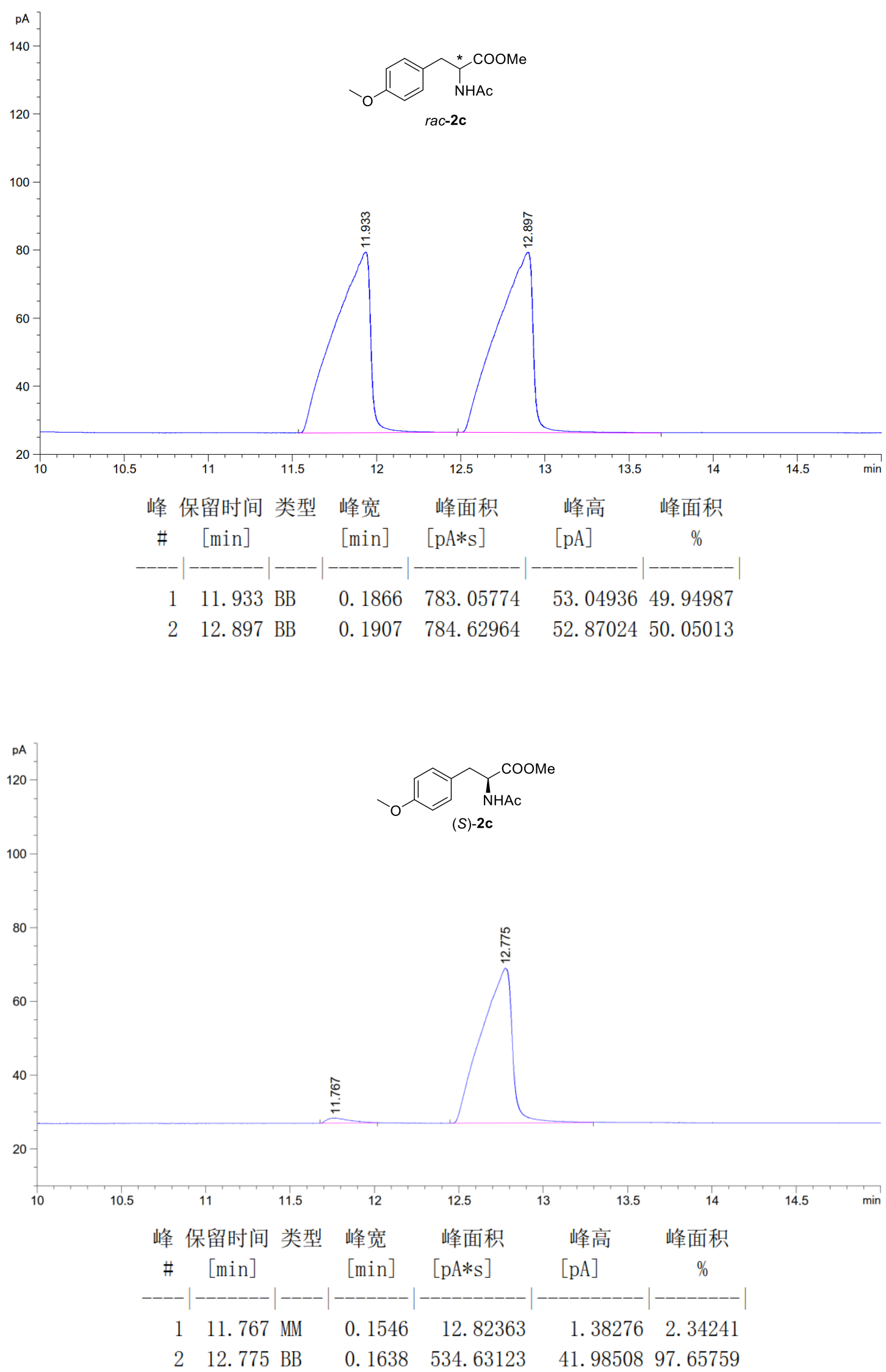


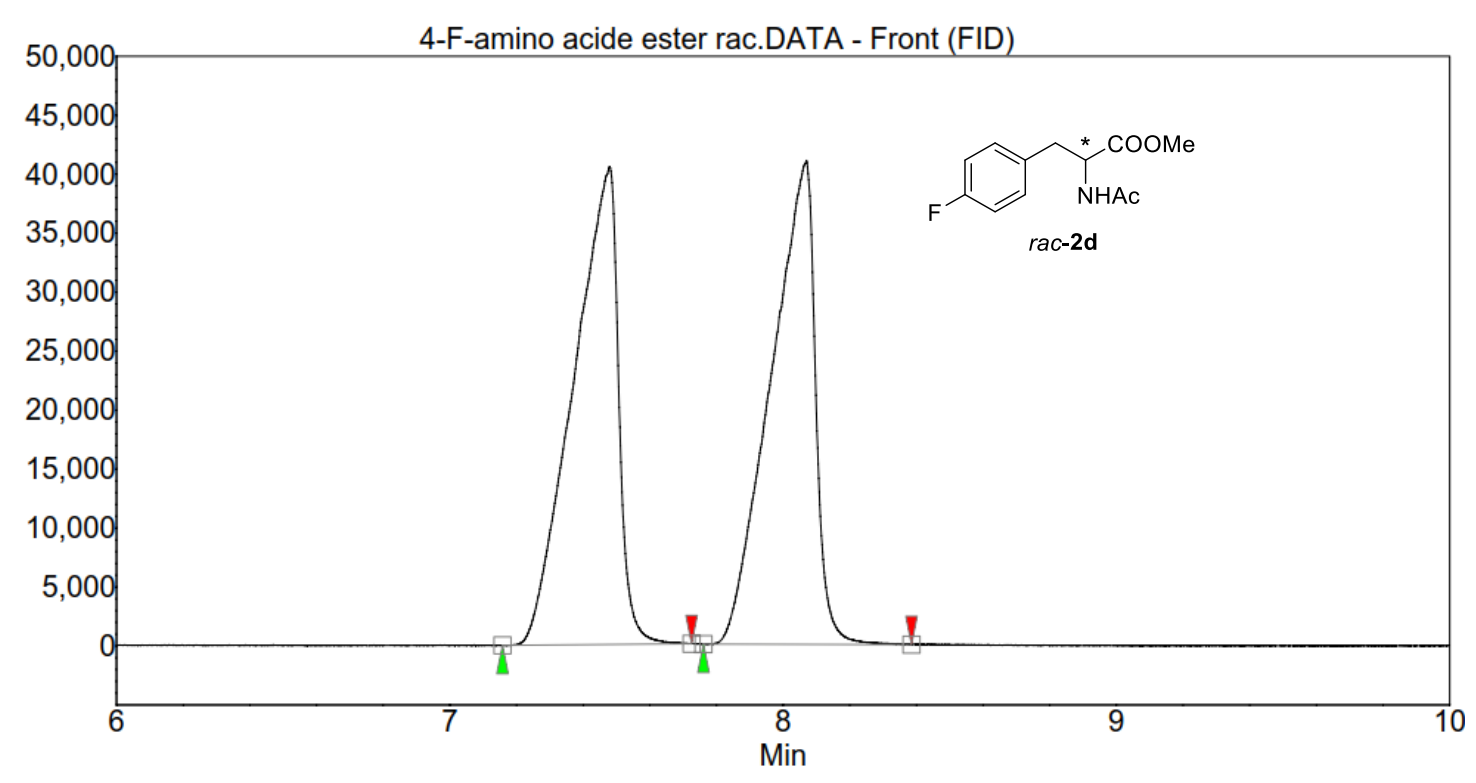

Peak results :

\begin{tabular}{|c|l|r|r|r|r|r|}
\hline Index & 文件名 & $\begin{array}{r}\text { 时间 } \\
\text { [Min] }\end{array}$ & $\begin{array}{r}\text { 数量 } \\
\text { [\% 面积] }\end{array}$ & $\begin{array}{r}\text { 高度 } \\
\text { [磺] }\end{array}$ & $\begin{array}{r}\text { Area } \\
\text { [磺.Min] }\end{array}$ & $\begin{array}{r}\text { Area \% } \\
{[\%]}\end{array}$ \\
\hline \hline 1 & 未知 & 7.48 & 49.93 & 40521.9 & 6333.2 & 49.926 \\
\hline 2 & 未知 & 8.07 & 50.07 & 41029.0 & 6352.0 & 50.074 \\
\hline & & & & & & \\
\hline Total & & & 100.00 & 81550.8 & 12685.2 & 100.000 \\
\hline
\end{tabular}

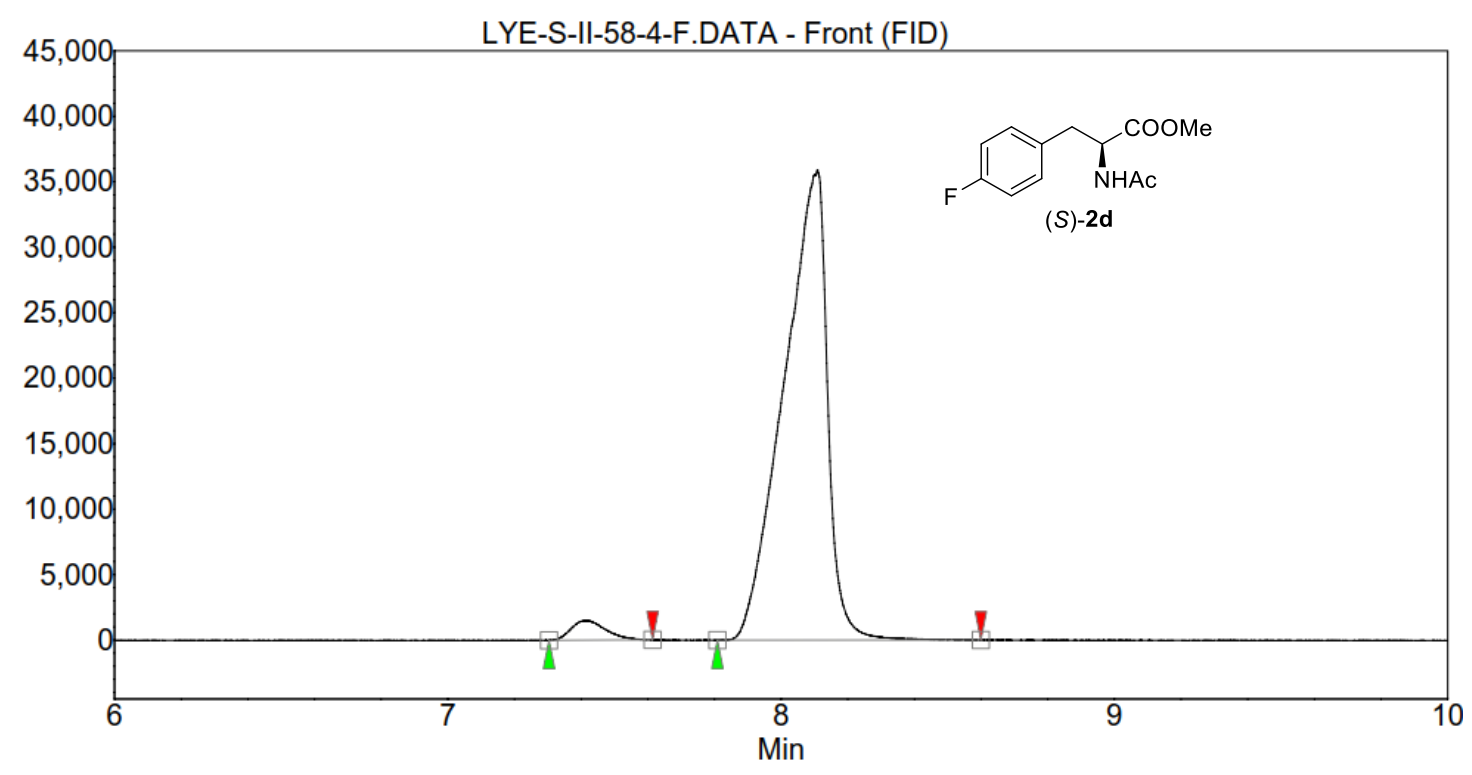

Peak results :

\begin{tabular}{|c|l|r|r|r|r|r|}
\hline Index & 文件名 & $\begin{array}{r}\text { 时间 } \\
\text { [Min] }\end{array}$ & $\begin{array}{r}\text { 数量 } \\
\text { [\% 面积] }\end{array}$ & $\begin{array}{r}\text { 高度 } \\
\text { [磺] }\end{array}$ & $\begin{array}{r}\text { Area } \\
\text { [磺.Min] }\end{array}$ & $\begin{array}{r}\text { Area \% } \\
\text { [\%] }\end{array}$ \\
\hline \hline 1 & 未知 & 7.41 & 3.18 & 1470.8 & 178.4 & 3.180 \\
\hline 2 & 未知 & 8.11 & 96.82 & 35911.4 & 5431.9 & 96.820 \\
\hline & & & & & & \\
\hline Total & & & 100.00 & 37382.2 & 5610.3 & 100.000 \\
\hline
\end{tabular}




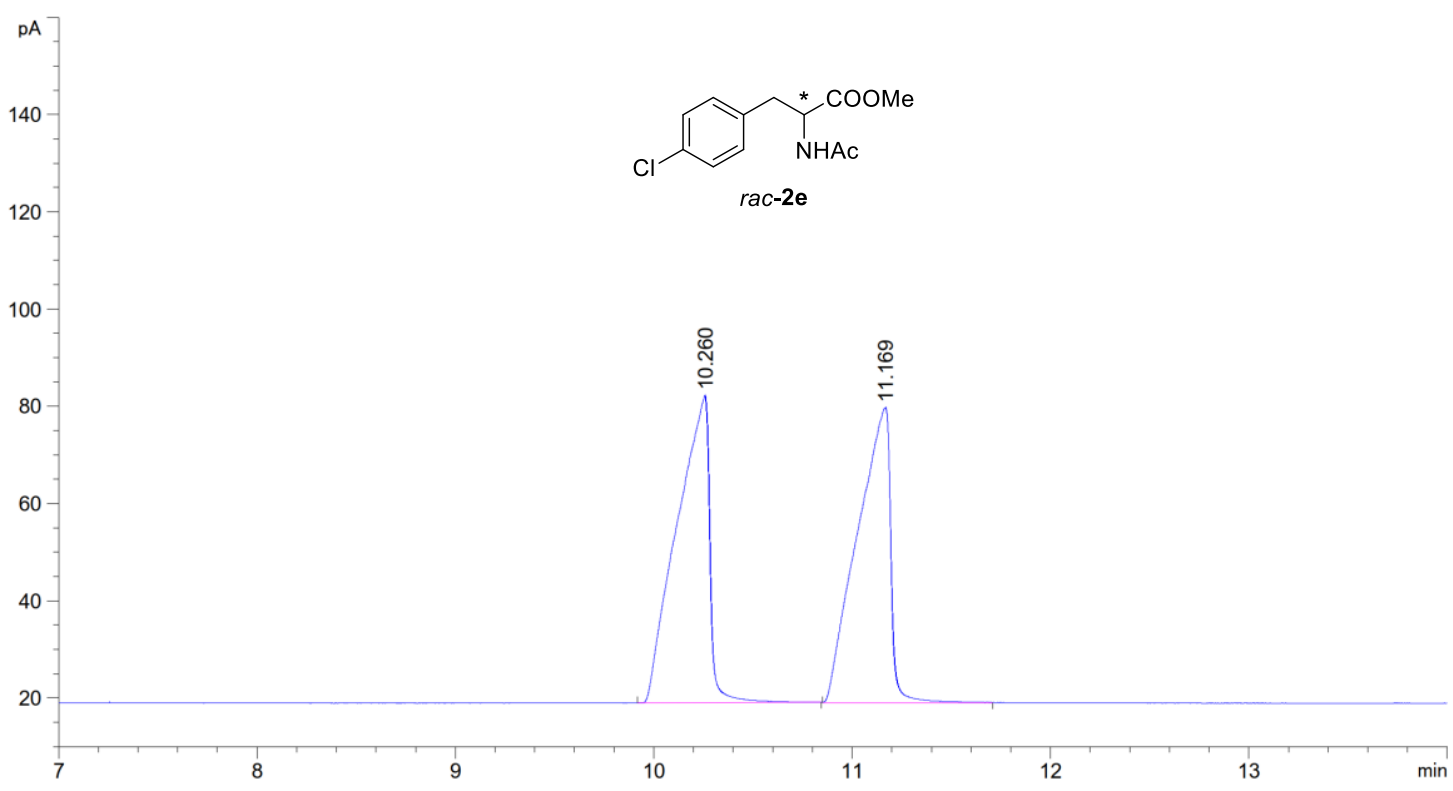

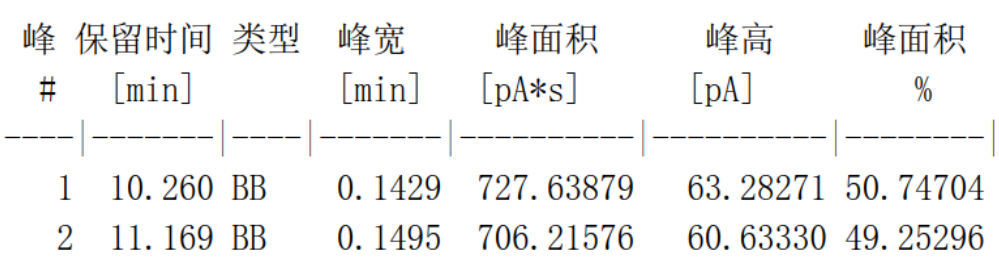

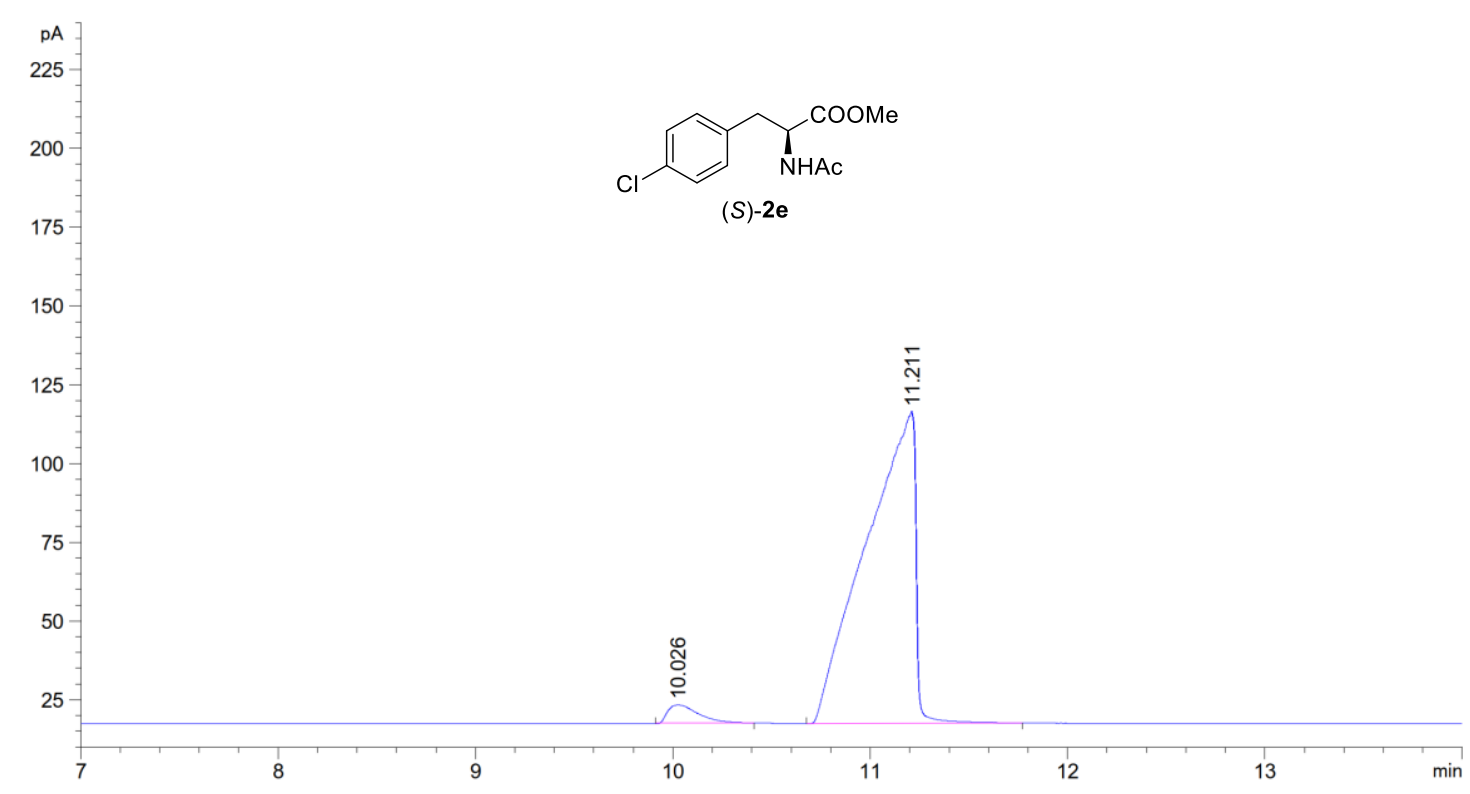

\begin{tabular}{|c|c|c|c|c|c|c|}
\hline 峰 & $\begin{array}{c}\text { 保留时间 } \\
\text { [min] }\end{array}$ & 类型 & $\begin{array}{l}\text { 峰宽 } \\
\text { [min] }\end{array}$ & $\begin{array}{l}\text { 峰面积 } \\
\text { [pA*s] }\end{array}$ & $\begin{array}{c}\text { 峰高 } \\
{[\mathrm{pA}]}\end{array}$ & $\begin{array}{c}\text { 峰面积 } \\
\%\end{array}$ \\
\hline & 10.0 & & 0.1817 & 63.52980 & 5. 82770 & 3. 54496 \\
\hline 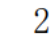 & 11.211 & $\mathrm{BB}$ & 0.2126 & 1728.58484 & 98.94540 & 96.45504 \\
\hline
\end{tabular}




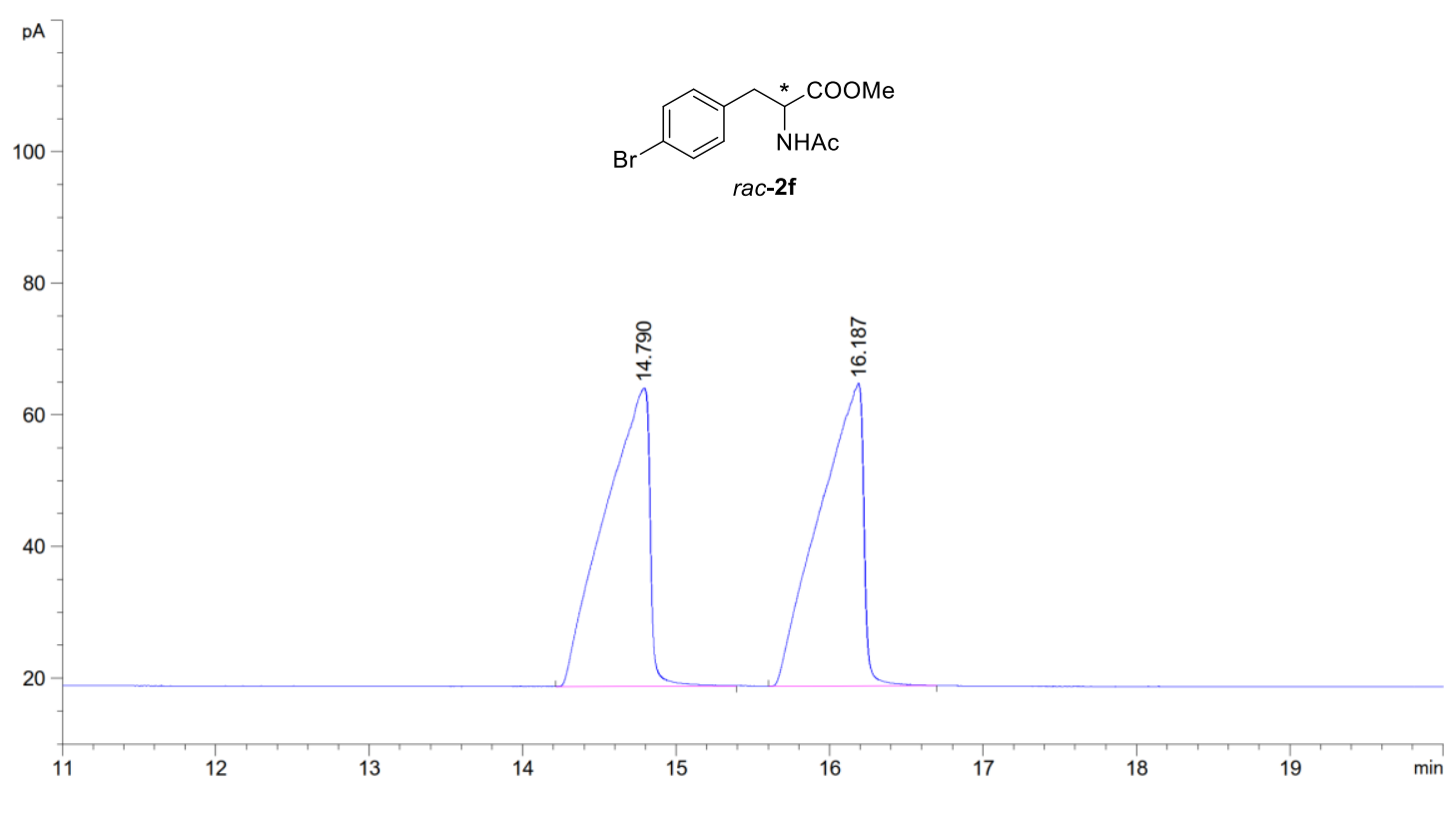

\begin{tabular}{|c|c|c|c|c|c|c|}
\hline 峰 & $\begin{array}{c}\text { 保留时间 } \\
\text { [min] }\end{array}$ & 类型 & $\begin{array}{l}\text { 峰宽 } \\
{[\mathrm{min}]}\end{array}$ & $\begin{array}{c}\text { 峰面积 } \\
{\left[\mathrm{pA} *_{\mathrm{s}}\right]}\end{array}$ & $\begin{array}{l}\text { 峰高 } \\
{[\mathrm{pA}]}\end{array}$ & $\begin{array}{c}\text { 峰面积 } \\
\%\end{array}$ \\
\hline & & & 504 & 917. & 3833 & 9436 \\
\hline & 16. $18^{\prime}$ & & 0.2462 & 913. 64734 & 45.98726 & 49. 9056 \\
\hline
\end{tabular}
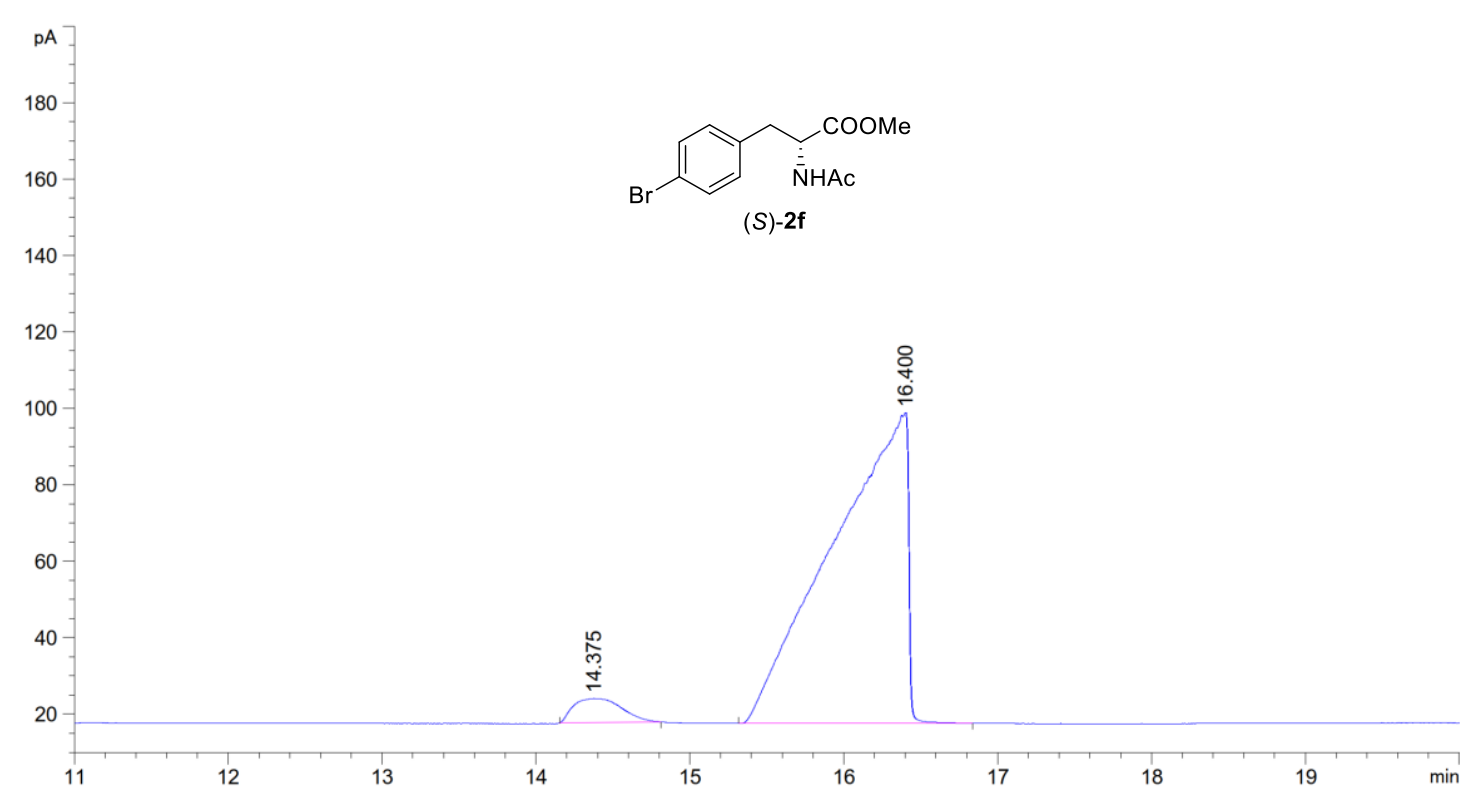

峰 保留时间 类型 峰宽 峰面积 峰高 峰面积

\begin{tabular}{cccccc}
$\#$ & {$[\mathrm{~min}]$} & {$[\mathrm{min}]$} & {$[\mathrm{pA}$ s $]$} & \multicolumn{1}{c}{$[\mathrm{pA}]$} & $\%$ \\
\hline 1 & $14.375 \mathrm{MM}$ & 0.3583 & 134.23007 & 6.24354 & 4.62722 \\
2 & $16.400 \mathrm{BB}$ & 0.4070 & 2766.64868 & 81.21313 & 95.37278
\end{tabular}



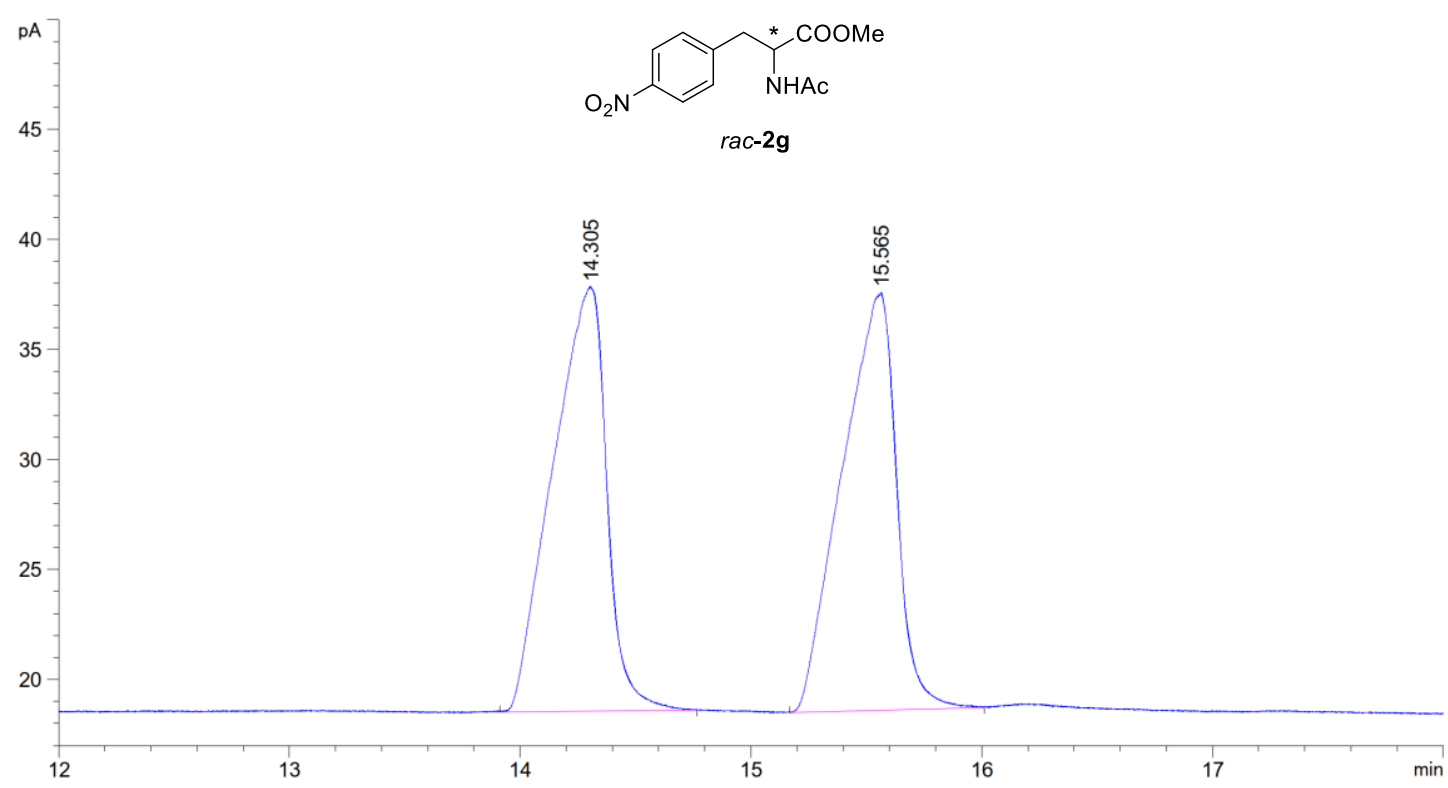

峰保留时间 类型 峰宽 峰面积峰高峰面积

$\begin{array}{cccccc}\# & {[\mathrm{~min}]} & {[\mathrm{min}]} & {[\mathrm{pA} * \mathrm{~s}]} & {[\mathrm{pA}]} & \% \\ -1 & 14.305 \text { BB } & 0.1945 & 313.61234 & 19.28912 & 50.16125 \\ 2 & 15.565 \text { BB } & 0.2020 & 311.59604 & 18.97433 & 49.83875\end{array}$

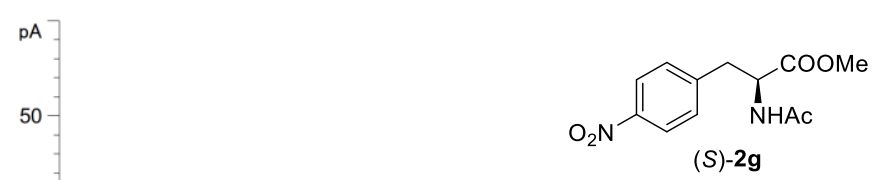

(S)-29

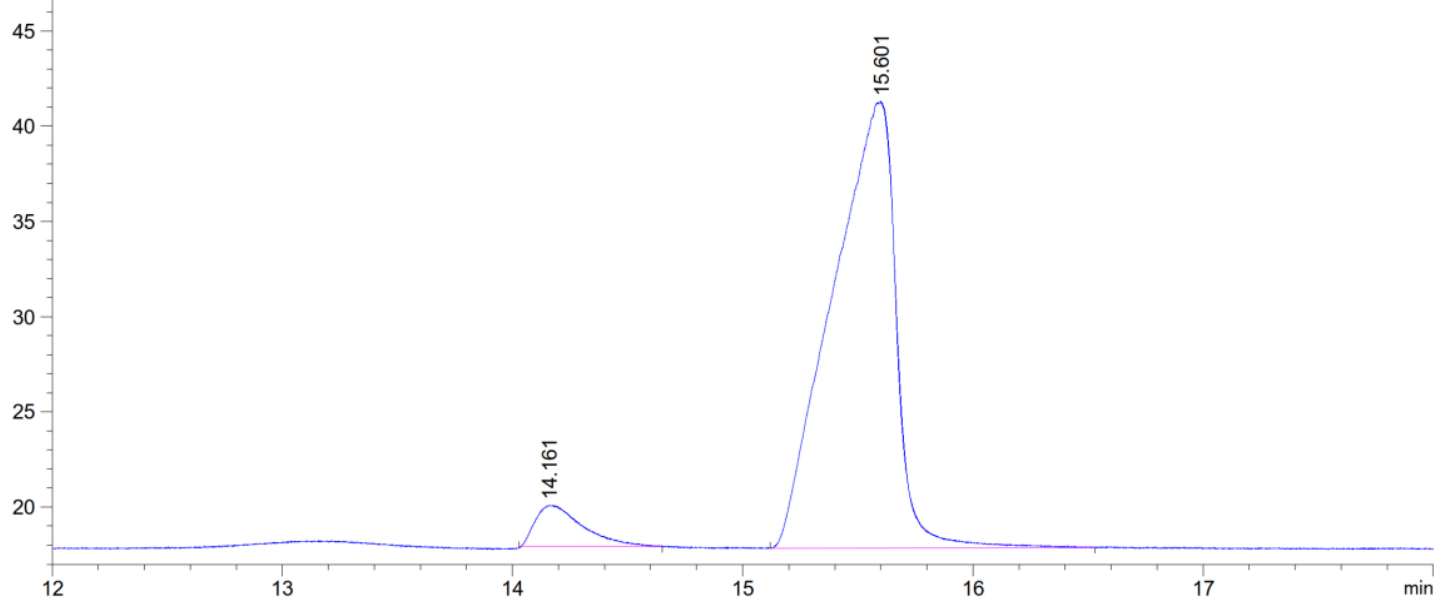

峰 保留时间 类型 峰宽峰面积峰高峰面积

\begin{tabular}{|c|c|c|c|c|c|}
\hline$\#$ & {$[\mathrm{~min}]$} & {$[\mathrm{min}]$} & {$[\mathrm{pA} * \mathrm{~s}]$} & {$[\mathrm{pA}]$} & $\%$ \\
\hline 1 & 14. $161 \mathrm{MM}$ & 0.2371 & 30.91110 & 2. 17293 & 6. 41810 \\
\hline 2 & 15. $601 \mathrm{MM}$ & 0.3205 & 450.71265 & 23. 43476 & 93.58190 \\
\hline
\end{tabular}



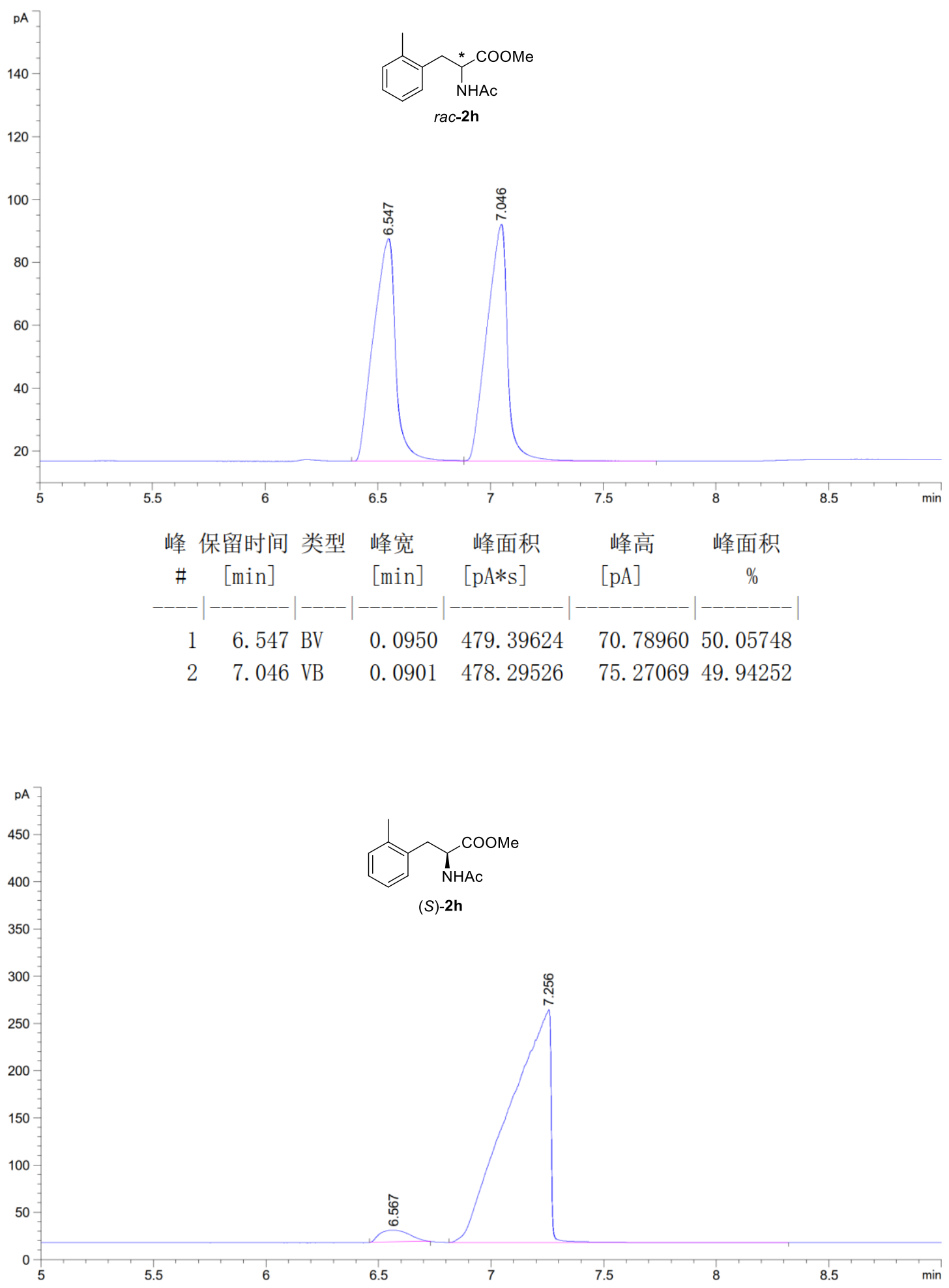

峰 保留时间类型峰宽峰面积峰高峰面积

\begin{tabular}{cccccc}
$\#$ & {$[\mathrm{~min}]$} & {$[\mathrm{min}]$} & {$[\mathrm{pA}$ *s $]$} & \multicolumn{1}{c}{$[\mathrm{pA}]$} & $\%$ \\
\hline 1 & 6.567 MM & 0.1530 & 113.65714 & 12.38358 & 3.43149 \\
2 & 7.256 MM & 0.2161 & 3198.52734 & 246.66089 & 96.56851
\end{tabular}




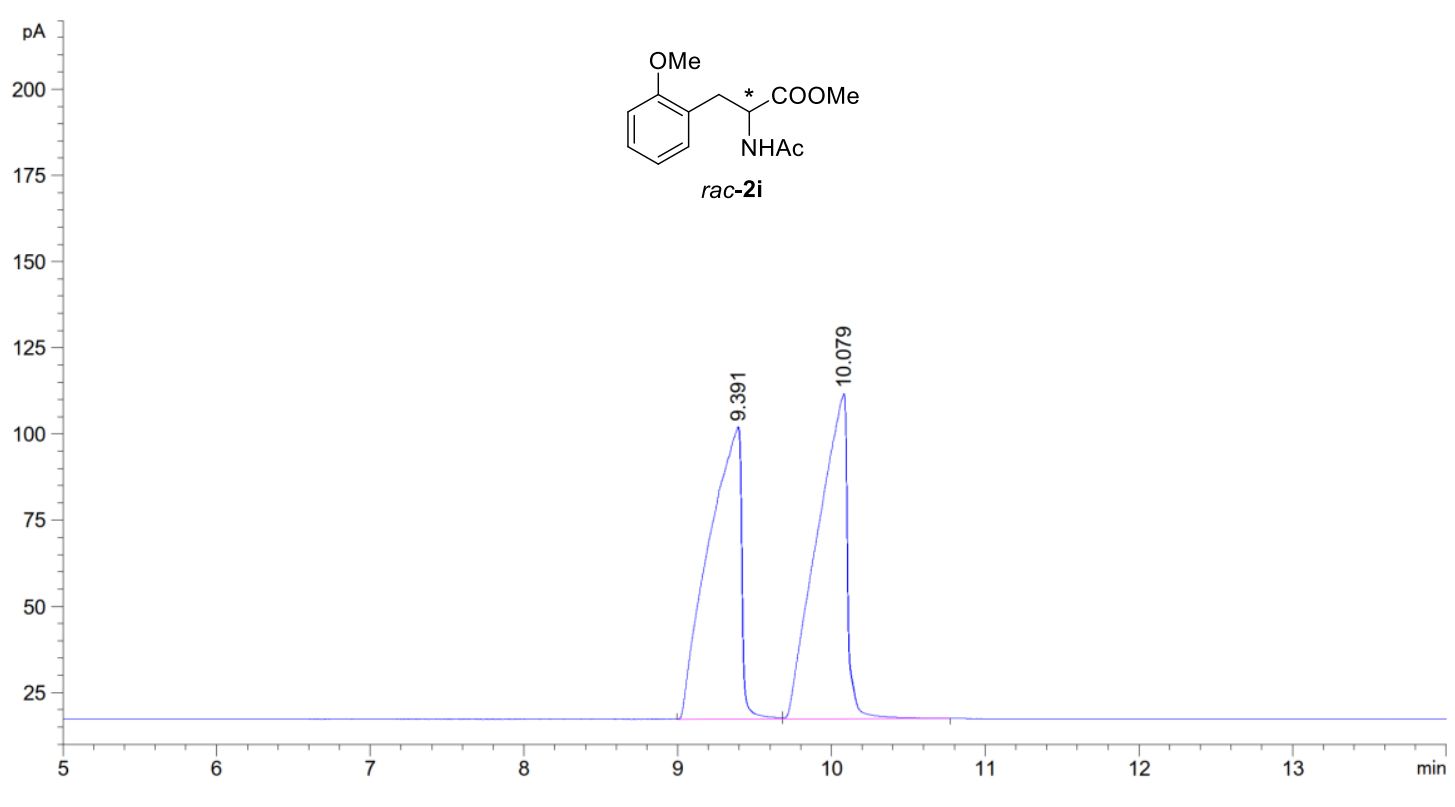

峰保留时间类型 峰宽 峰面积 峰高峰面积

\begin{tabular}{crcccc}
$\#$ & {$[\mathrm{~min}]$} & {$[\mathrm{min}]$} & {$[\mathrm{pA} * \mathrm{~s}]$} & {$[\mathrm{pA}]$} & $\%$ \\
\hline 1 & $9.391 \mathrm{BV}$ & 0.1817 & 1250.81128 & 84.77244 & 49.35052 \\
2 & $10.079 \mathrm{VB}$ & 0.1668 & 1283.73425 & 94.29718 & 50.64948
\end{tabular}

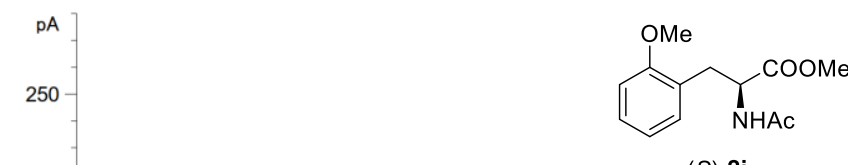

(S)-2 $\mathbf{i}$

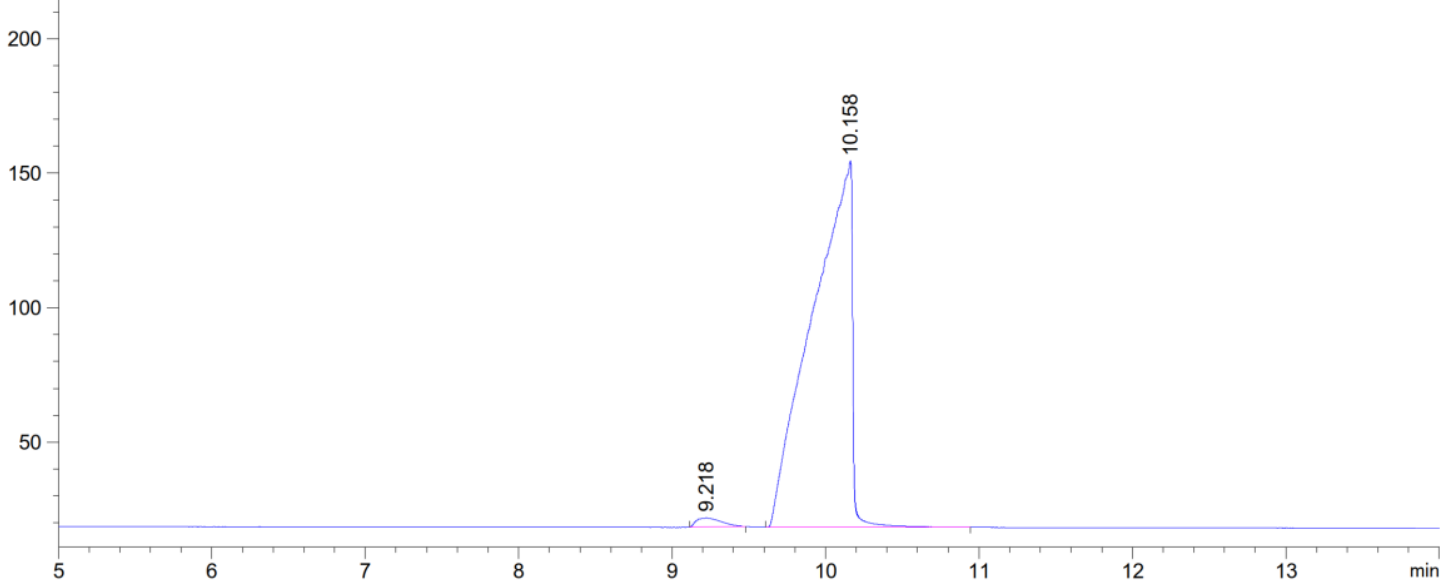

\begin{tabular}{|c|c|c|c|c|c|c|}
\hline $\begin{array}{r}\text { 峰 } \\
\#\end{array}$ & $\begin{array}{c}\text { 保留时间 } \\
\text { [min] }\end{array}$ & 类型 & $\begin{array}{l}\text { 峰宽 } \\
\text { [min] }\end{array}$ & $\begin{array}{l}\text { 峰面积 } \\
{[\mathrm{pA} * \mathrm{~s}]}\end{array}$ & $\begin{array}{c}\text { 峰高 } \\
{[\mathrm{pA}]}\end{array}$ & $\begin{array}{c}\text { 峰面积 } \\
\%\end{array}$ \\
\hline 1 & 9.218 & MM & 0.1873 & 38.26013 & 3. 40378 & 1. 52926 \\
\hline 2 & 10. 158 & $\mathrm{BB}$ & 0.2186 & 2463. 60596 & 135.98300 & 98. 47074 \\
\hline
\end{tabular}



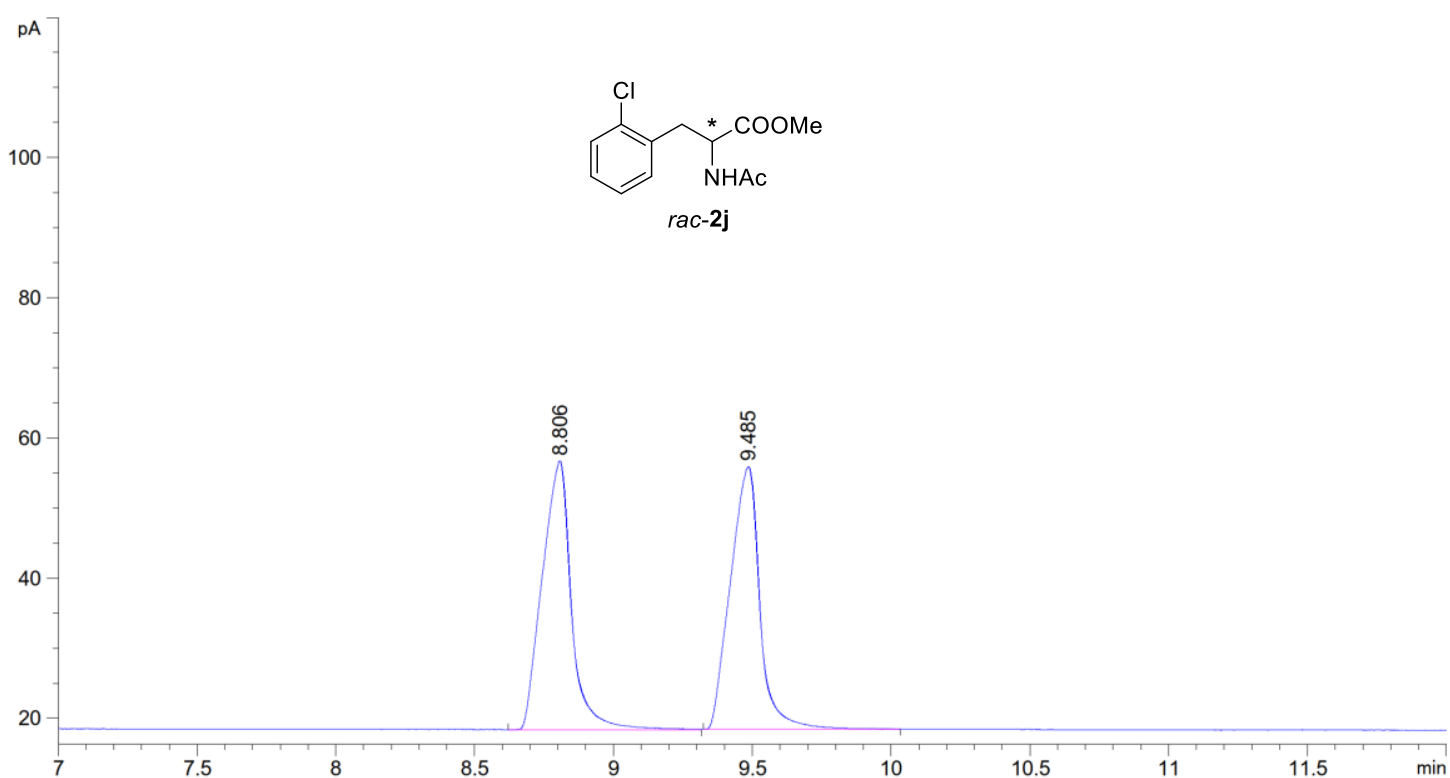

峰 保留时间 类型 峰宽峰面积峰高峰面积

\begin{tabular}{cccccc}
$\#$ & {$[\mathrm{~min}]$} & {$[\mathrm{min}]$} & {$\left[\mathrm{pA} *_{\mathrm{s}}\right]$} & {$[\mathrm{pA}]$} & $\%$ \\
\hline 1 & 8.806 BB & 0.0995 & 279.46207 & 38.33184 & 50.28126 \\
2 & 9.485 BB & 0.0989 & 276.33563 & 37.44623 & 49.71874
\end{tabular}

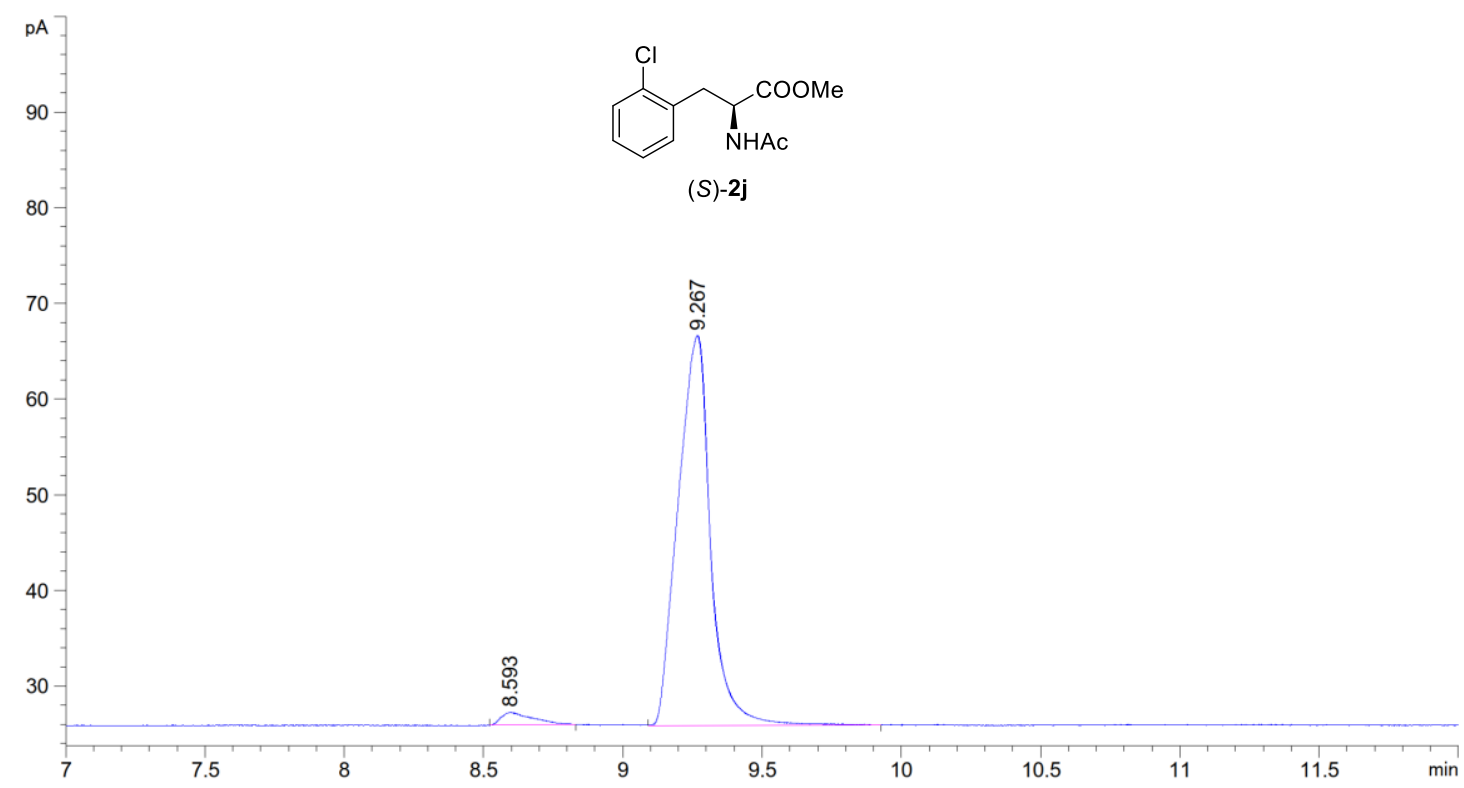

峰保留时间 类型 峰宽峰面积峰高峰面积

\begin{tabular}{cccccc}
$\#$ & {$[\mathrm{~min}]$} & {$[\mathrm{min}]$} & {$[\mathrm{pA} * \mathrm{~s}]$} & \multicolumn{1}{c}{$[\mathrm{pA}]$} & $\%$ \\
\hline 1 & 8.593 MM & 0.1254 & 9.89358 & 1.31463 & 2.90502 \\
2 & 9.267 BB & 0.1148 & 330.67526 & 40.70329 & 97.09498
\end{tabular}




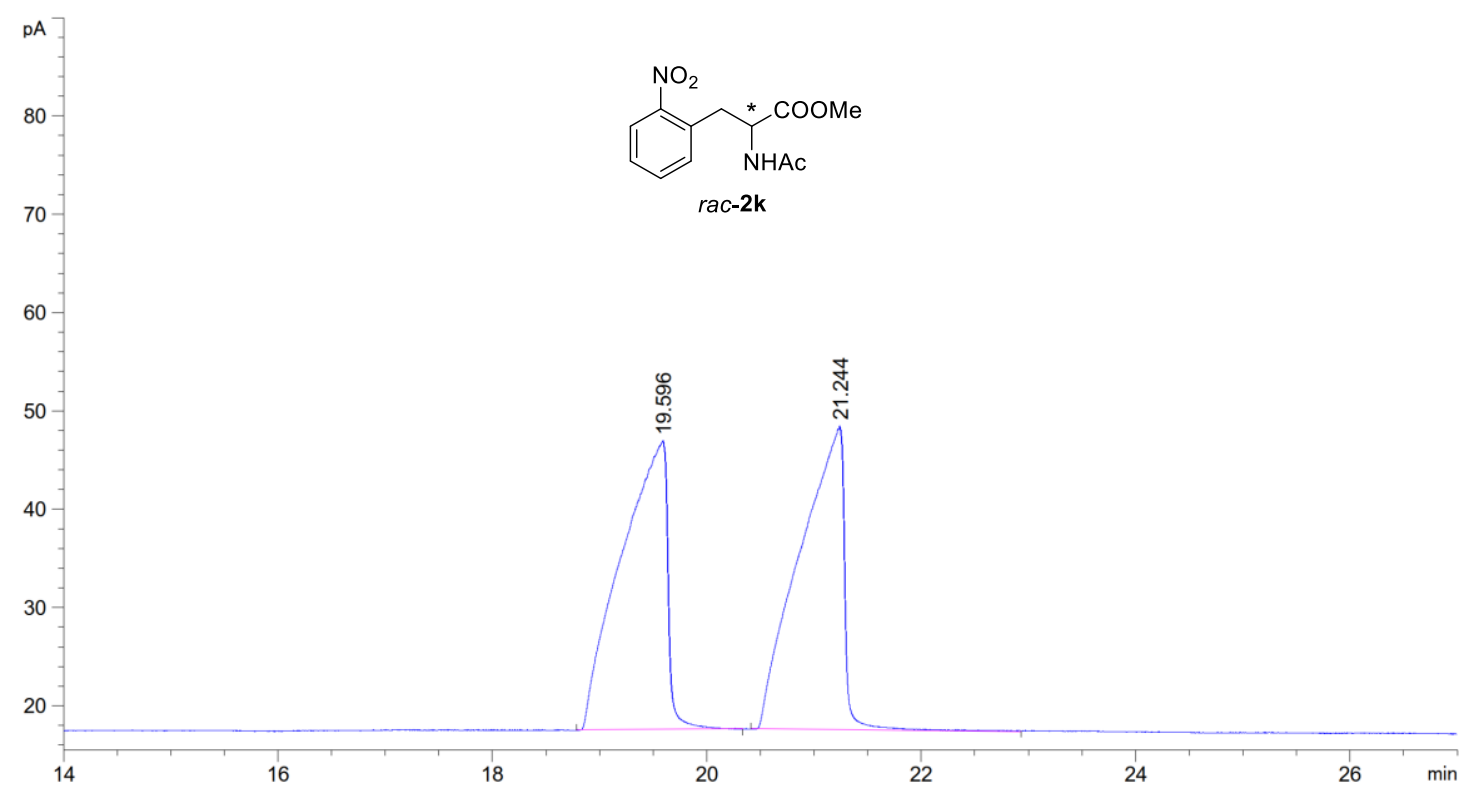

\begin{tabular}{|c|c|c|c|c|c|c|}
\hline$\#$ & $\begin{array}{c}\text { 保留时间 } \\
\text { [min] }\end{array}$ & 类型 & $\begin{array}{l}\text { 峰宽 } \\
{[\mathrm{min}]}\end{array}$ & $\begin{array}{c}\text { 峰面积 } \\
{\left[\mathrm{pA} *_{\mathrm{s}}\right]}\end{array}$ & $\begin{array}{l}\text { 峰高 } \\
{[\mathrm{pA}]}\end{array}$ & $\begin{array}{c}\text { 峰面积 } \\
\%\end{array}$ \\
\hline & & & 0. & 878 & 671 & 7243 \\
\hline & 21.2 & & 0.4802 & 890.18488 & 30.89749 & 50.3275 \\
\hline
\end{tabular}

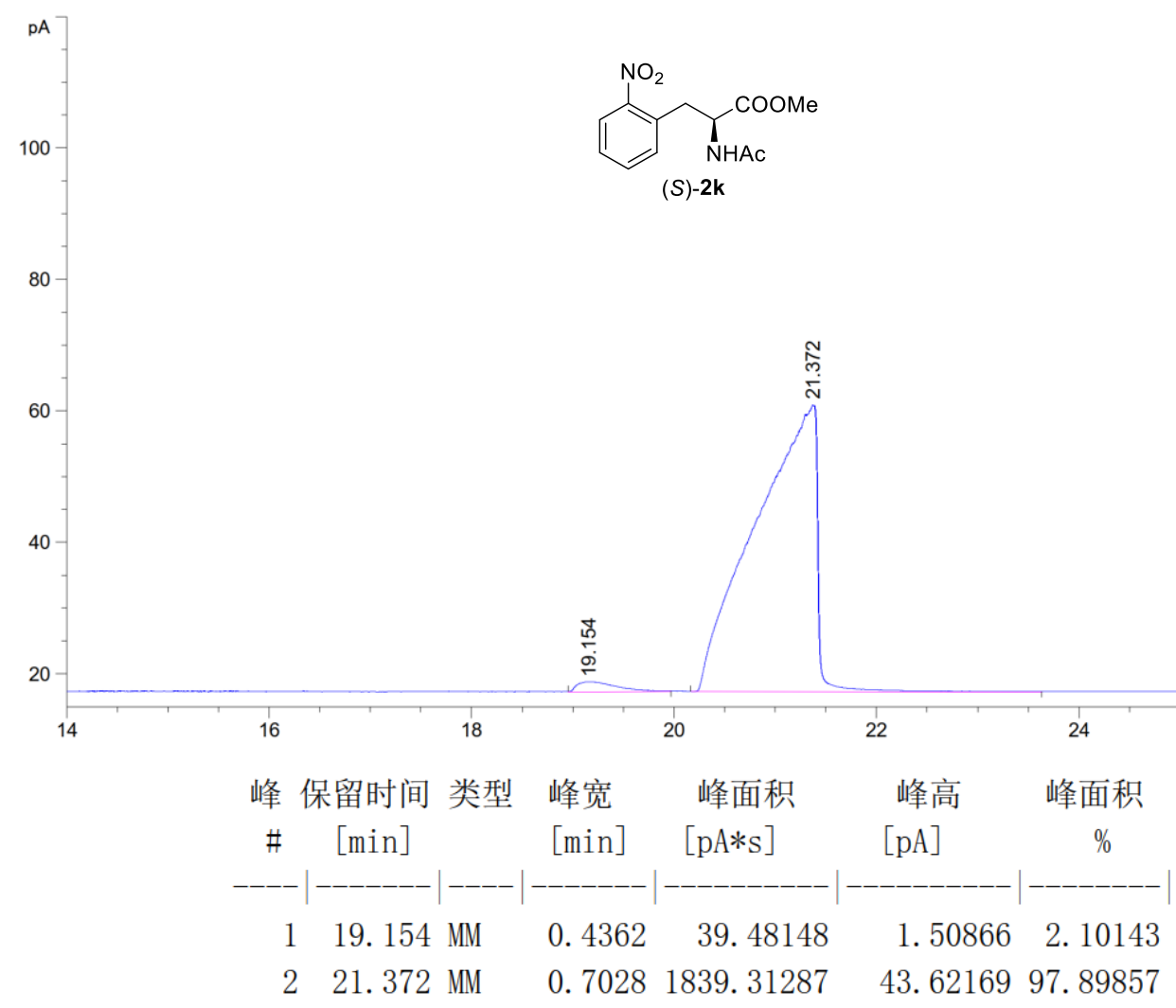




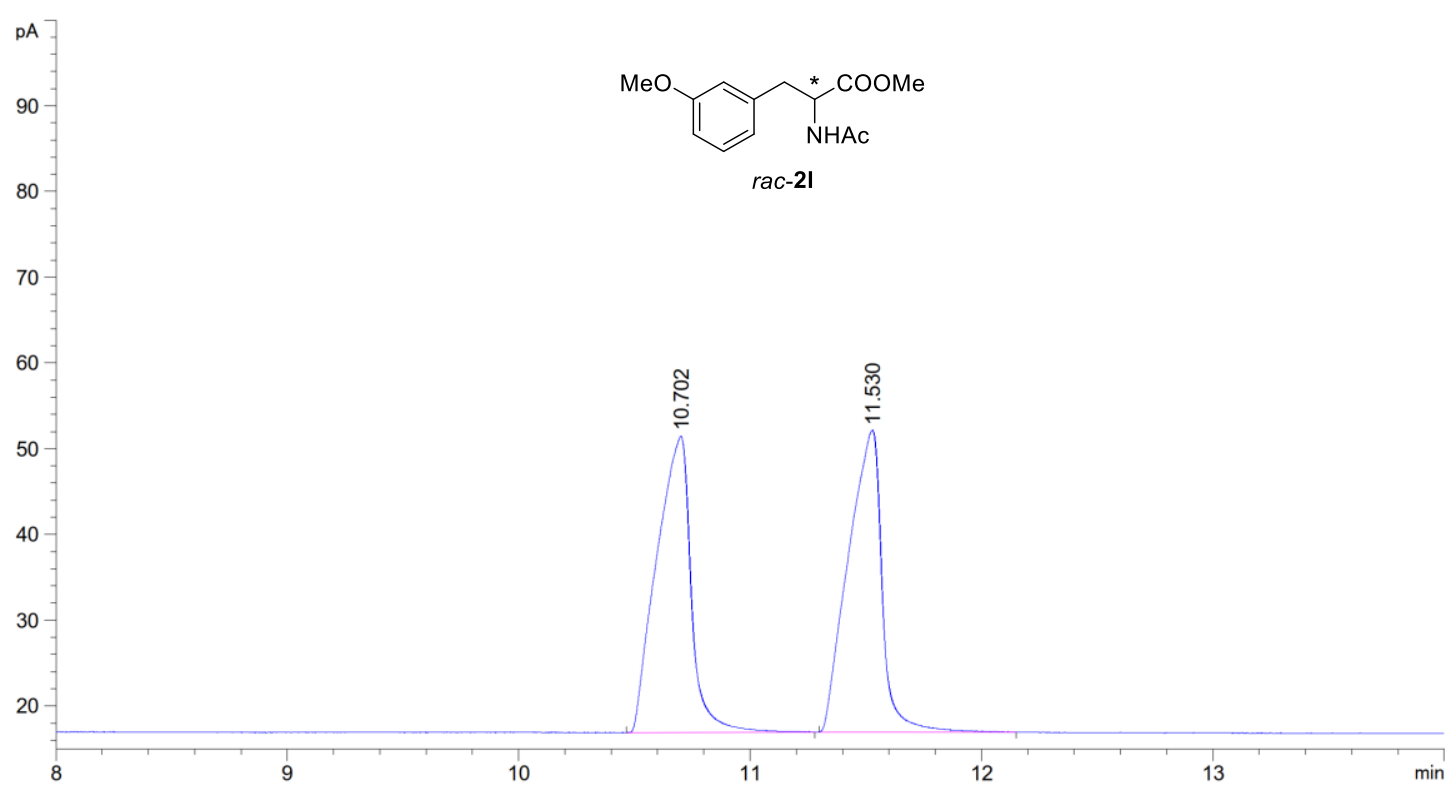

\begin{tabular}{|c|c|c|c|c|c|c|}
\hline 峰 & $\begin{array}{c}\text { 保留时间 } \\
\text { [min] }\end{array}$ & 类型 & $\begin{array}{l}\text { 峰宽 } \\
\text { [min] }\end{array}$ & $\begin{array}{c}\text { 峰面积 } \\
{\left[\mathrm{pA} *_{\mathrm{s}}\right]}\end{array}$ & $\begin{array}{c}\text { 峰高 } \\
{[\mathrm{pA}]}\end{array}$ & $\begin{array}{c}\text { 峰面积 } \\
\%\end{array}$ \\
\hline 1 & $10.7 \mathrm{C}$ & & 0.1356 & 359. 09848 & 34. 57588 & 0.10099 \\
\hline ? & 11.530 & 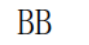 & 0.1337 & 357.65076 & 35.20985 & 49. 89901 \\
\hline
\end{tabular}

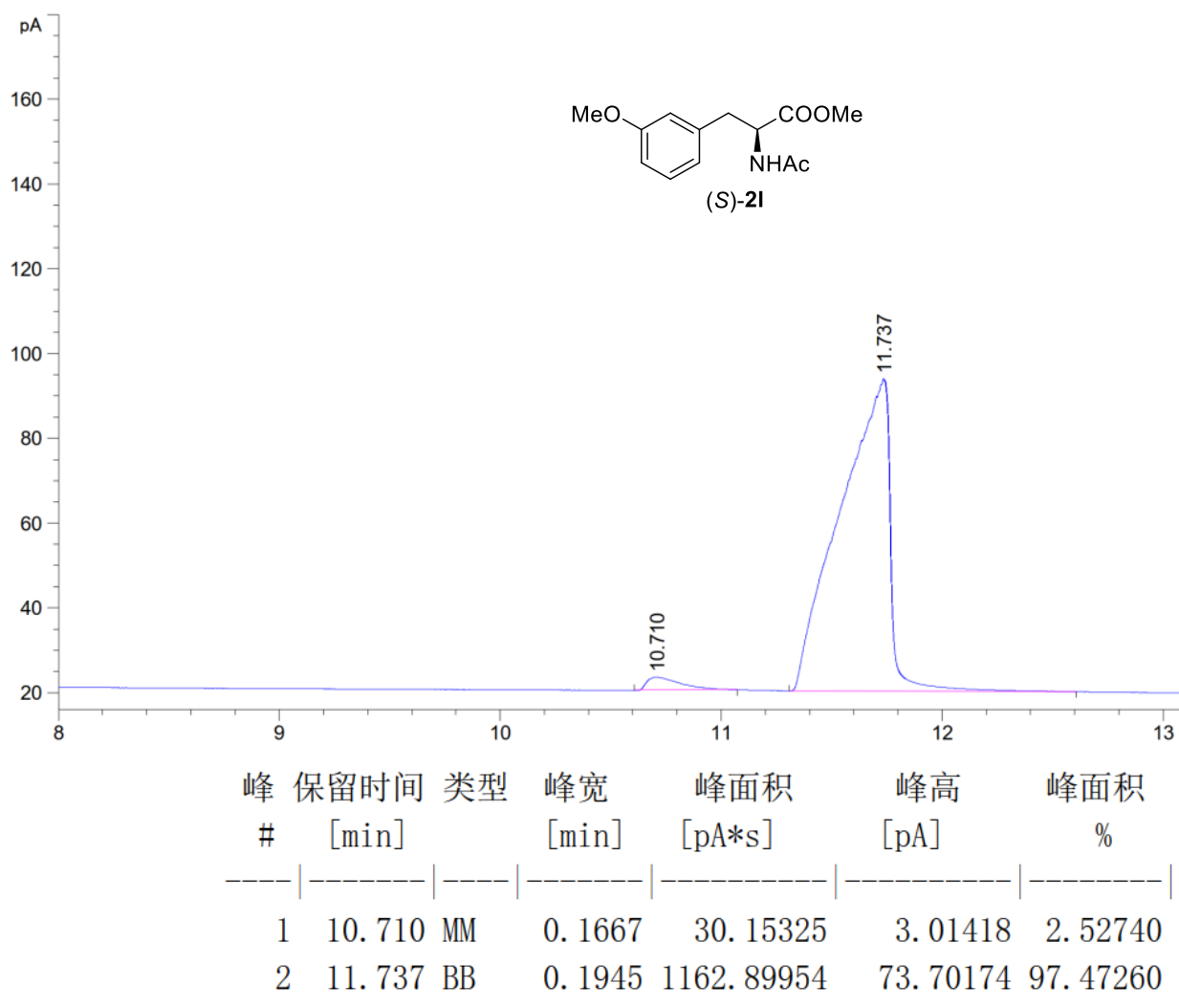




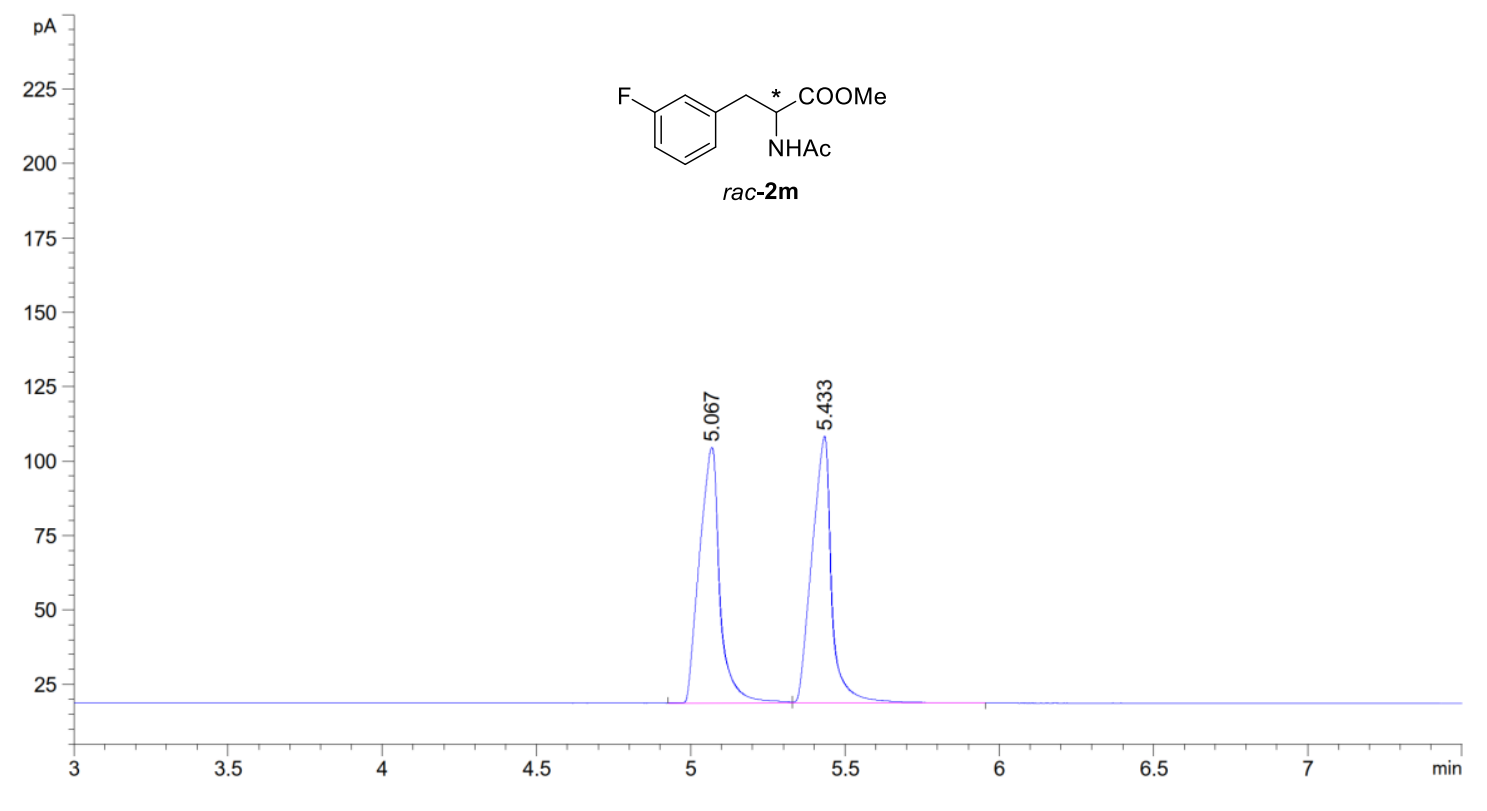

\begin{tabular}{|c|c|c|c|c|c|c|}
\hline 圣 & $\begin{array}{c}\text { 保留时间 } \\
\text { [min] }\end{array}$ & 类型 & $\begin{array}{l}\text { 峰宽 } \\
\text { [min] }\end{array}$ & $\begin{array}{c}\text { 峰面积 } \\
{\left[\mathrm{pA} *_{\mathrm{s}}\right]}\end{array}$ & $\begin{array}{c}\text { 峰高 } \\
{[\mathrm{pA}]}\end{array}$ & $\begin{array}{c}\text { 峰面积 } \\
\%\end{array}$ \\
\hline & & & 0.0747 & 396. & 380 & 8262 \\
\hline & 5.433 & & 0.0655 & 398. 33578 & 89. 56658 & 50.1173 \\
\hline
\end{tabular}

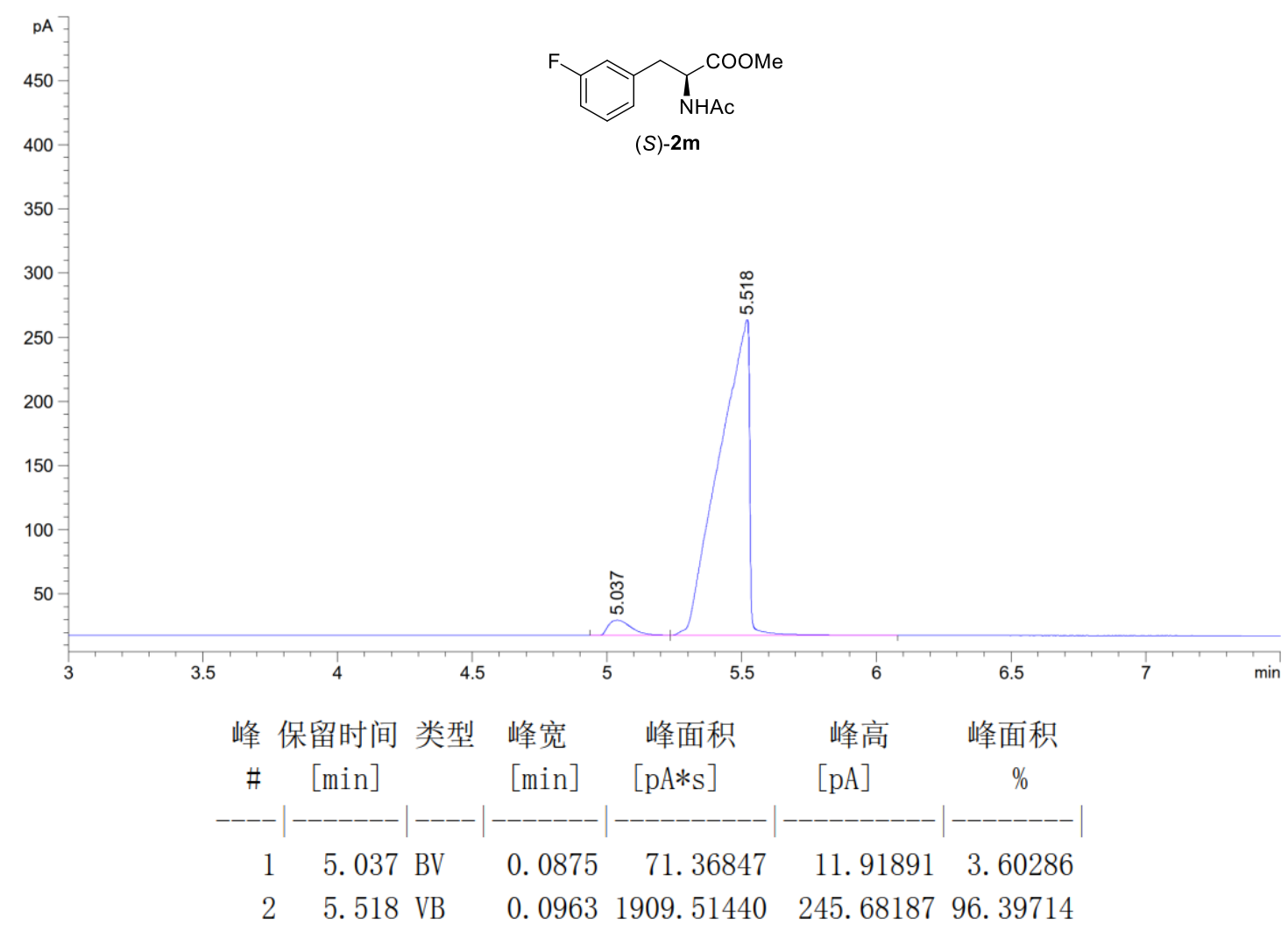




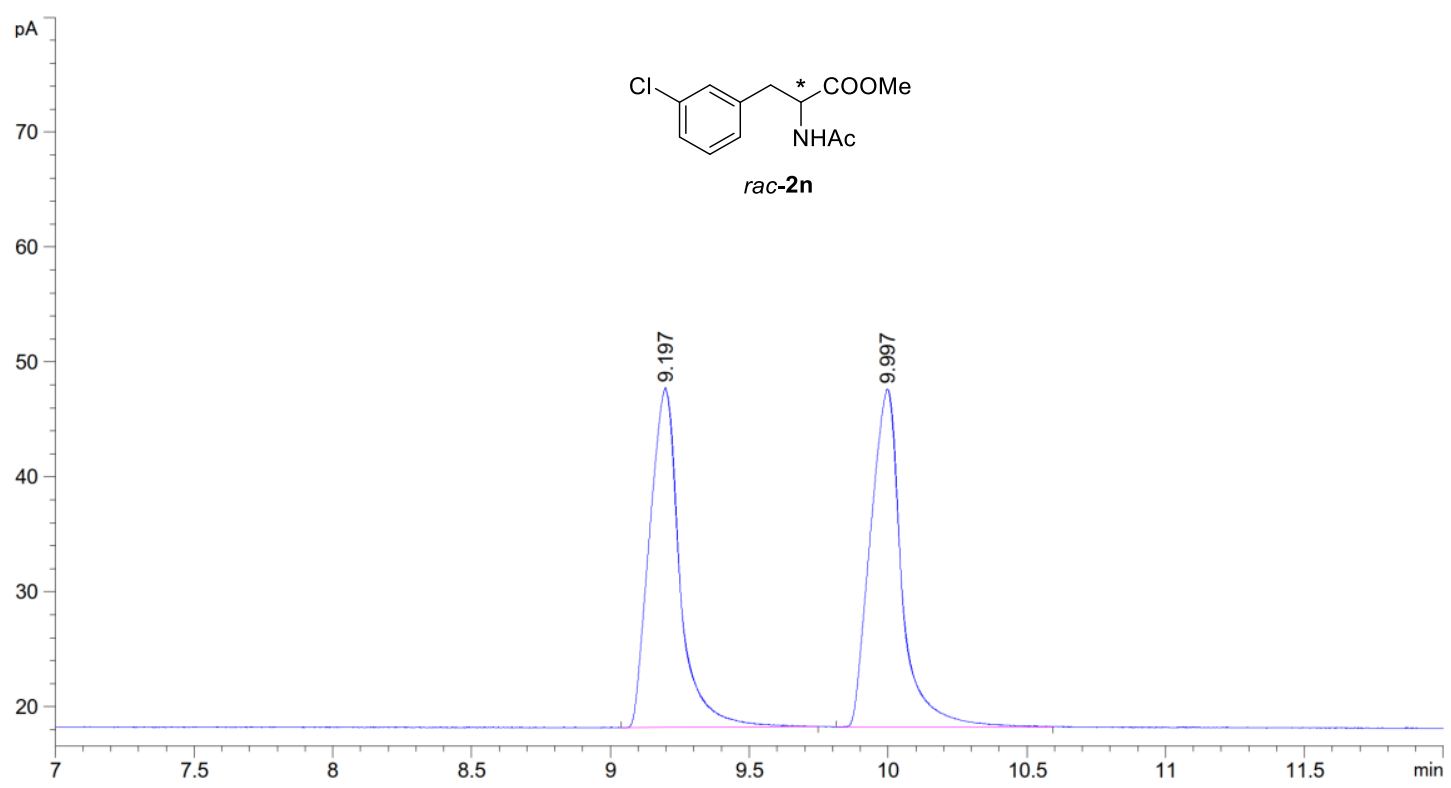

峰 保留时间 类型 峰宽 峰面积峰高 峰面积

\begin{tabular}{cccccc}
$\#$ & {$[\mathrm{~min}]$} & {$[\mathrm{min}]$} & {$[\mathrm{pA}$ s $]$} & {$[\mathrm{pA}]$} & $\%$ \\
\hline 1 & $9.197 \mathrm{BB}$ & 0.1078 & 224.54764 & 29.56320 & 49.30405 \\
2 & 9.997 BB & 0.1092 & 230.88683 & 29.41247 & 50.69595
\end{tabular}

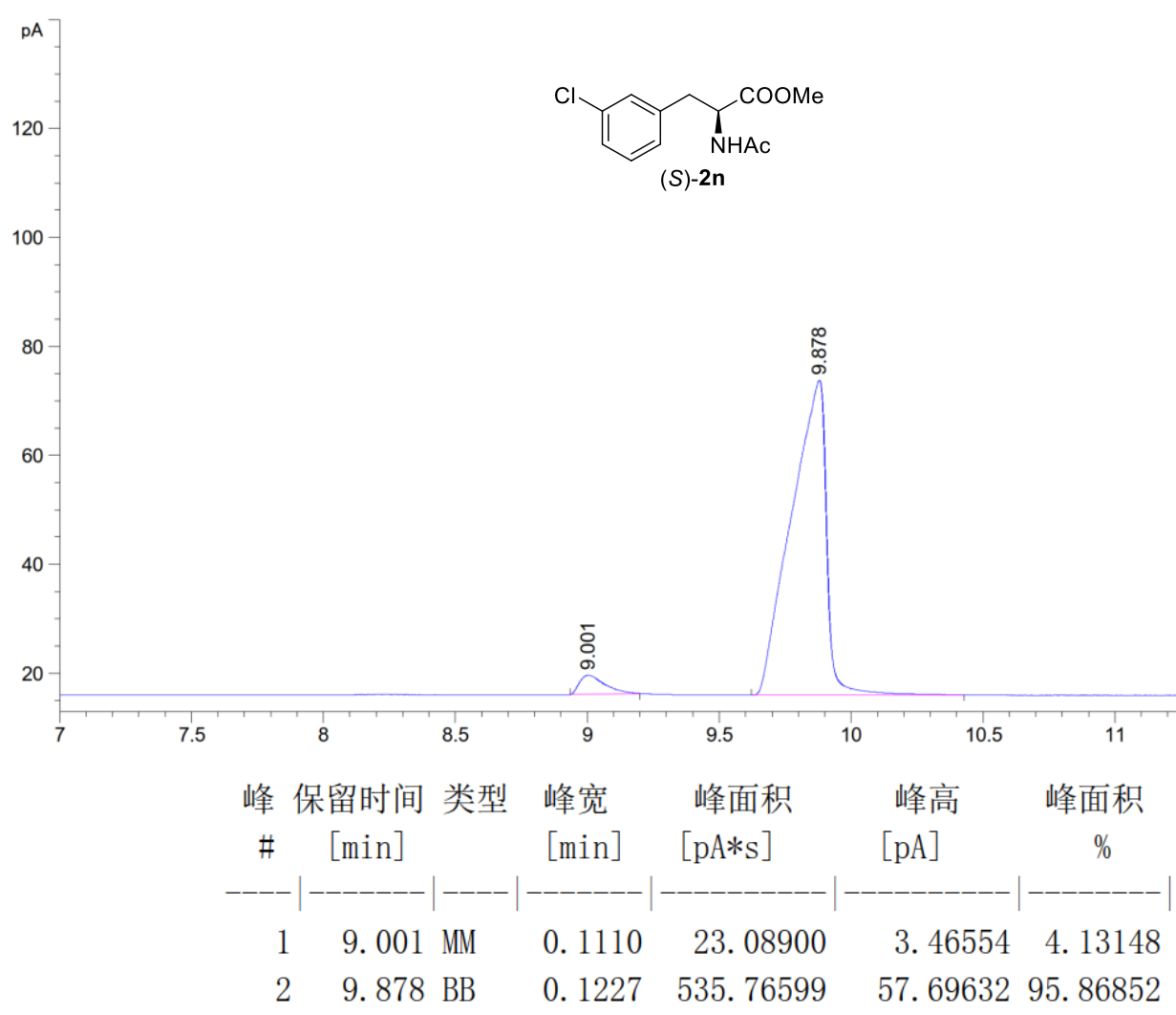




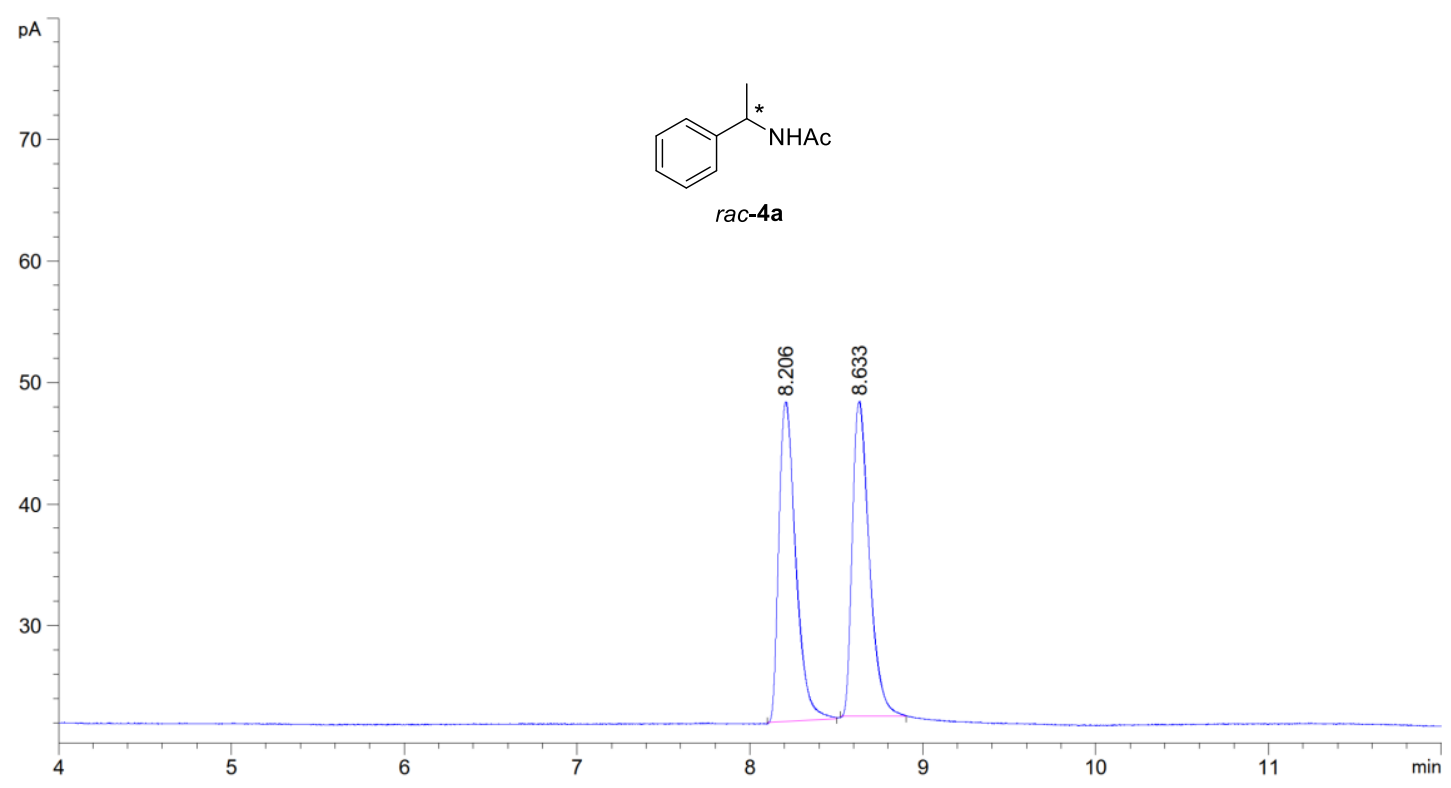

\begin{tabular}{|c|c|c|c|c|c|c|}
\hline 峰 & $\begin{array}{c}\text { 保留时间 } \\
\text { [min] }\end{array}$ & 类型 & $\begin{array}{l}\text { 峰宽 } \\
\text { [min] }\end{array}$ & $\begin{array}{c}\text { 峰面积 } \\
{\left[\mathrm{pA} *_{\mathrm{s}}\right]}\end{array}$ & $\begin{array}{l}\text { 峰高 } \\
{[\mathrm{pA}]}\end{array}$ & $\begin{array}{c}\text { 峰面积 } \\
\%\end{array}$ \\
\hline & & & 0.1133 & & & \\
\hline & 8. 6 & & 0.1136 & 176. 86096 & 25.94208 & 49. 73467 \\
\hline
\end{tabular}
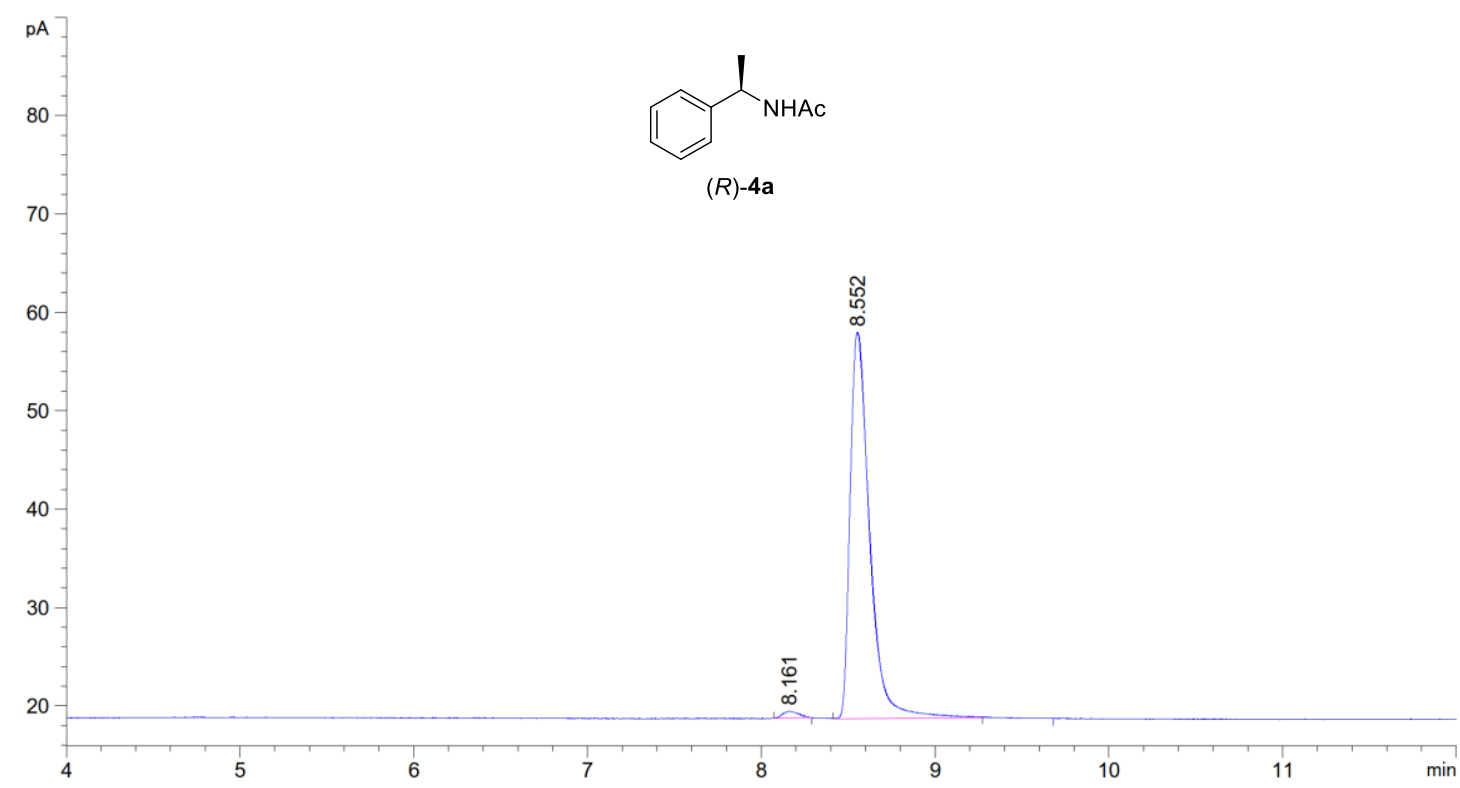

\begin{tabular}{|c|c|c|c|c|c|c|}
\hline 峰 & $\begin{array}{c}\text { 保留时间 } \\
\text { [min] }\end{array}$ & 类型 & $\begin{array}{l}\text { 峰宽 } \\
\text { [min] }\end{array}$ & $\begin{array}{l}\text { 峰面积 } \\
\text { [pA*s] }\end{array}$ & $\begin{array}{l}\text { 峰高 } \\
{[\mathrm{pA}]}\end{array}$ & $\begin{array}{c}\text { 峰面积 } \\
\%\end{array}$ \\
\hline & & & 0.1073 & 61 & 6.991 & 53 \\
\hline & 8.552 & & 0.1067 & 305. 11102 & 39. 24563 & 98.5463 \\
\hline
\end{tabular}




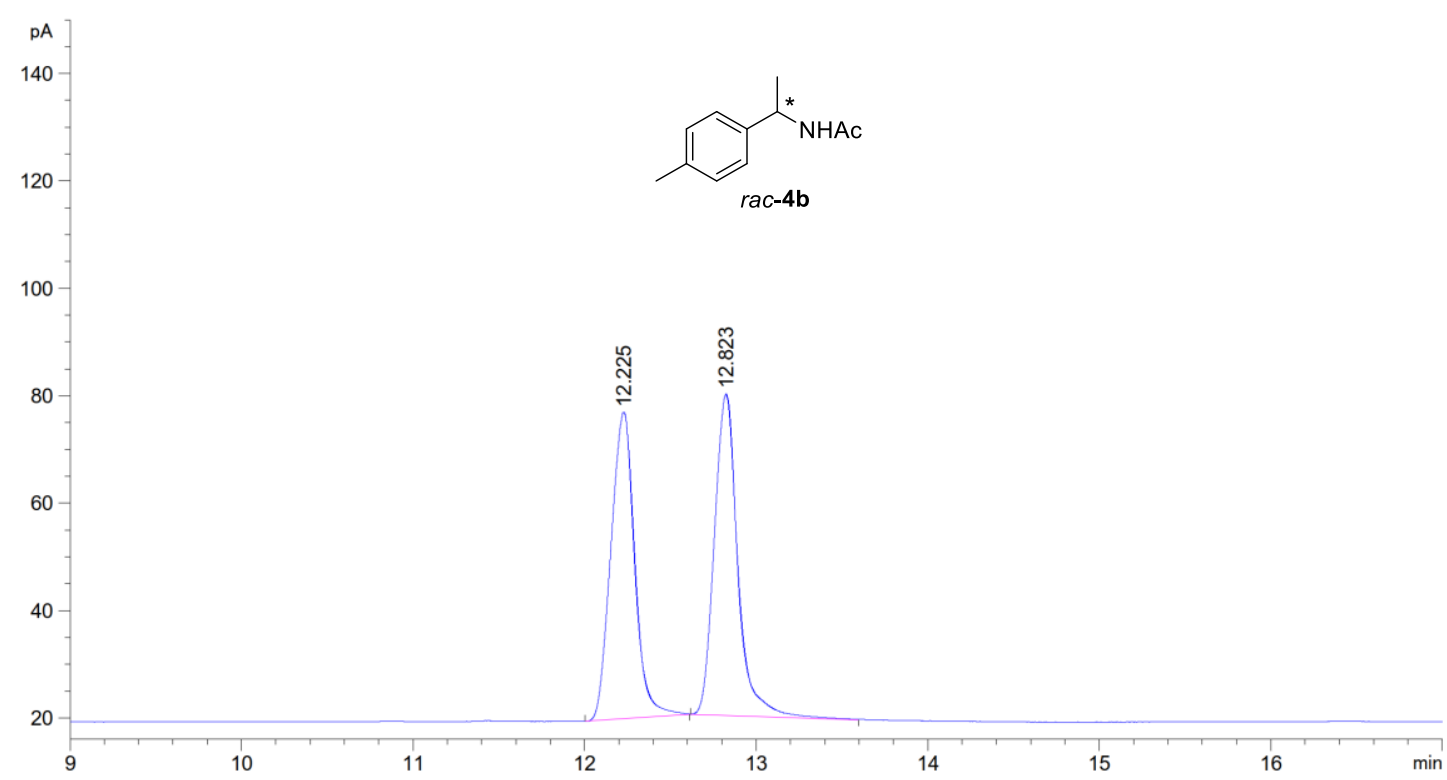

\begin{tabular}{|c|c|c|c|c|c|c|}
\hline & $\begin{array}{c}\text { 保留时间 } \\
\text { [min] }\end{array}$ & 类型 & $\begin{array}{l}\text { 峰宽 } \\
\text { [min] }\end{array}$ & $\begin{array}{l}\text { 峰面积 } \\
{\left[\mathrm{pA} *_{\mathrm{s}}\right]}\end{array}$ & $\begin{array}{l}\text { 峰高 } \\
{[\mathrm{pA}]}\end{array}$ & $\begin{array}{c}\text { 峰面积 } \\
\%\end{array}$ \\
\hline & & & 0.1386 & 548 & & 97 \\
\hline & 12.8 & & 0.1312 & 566. 14020 & 59.82830 & $50.776 \mathrm{C}$ \\
\hline
\end{tabular}

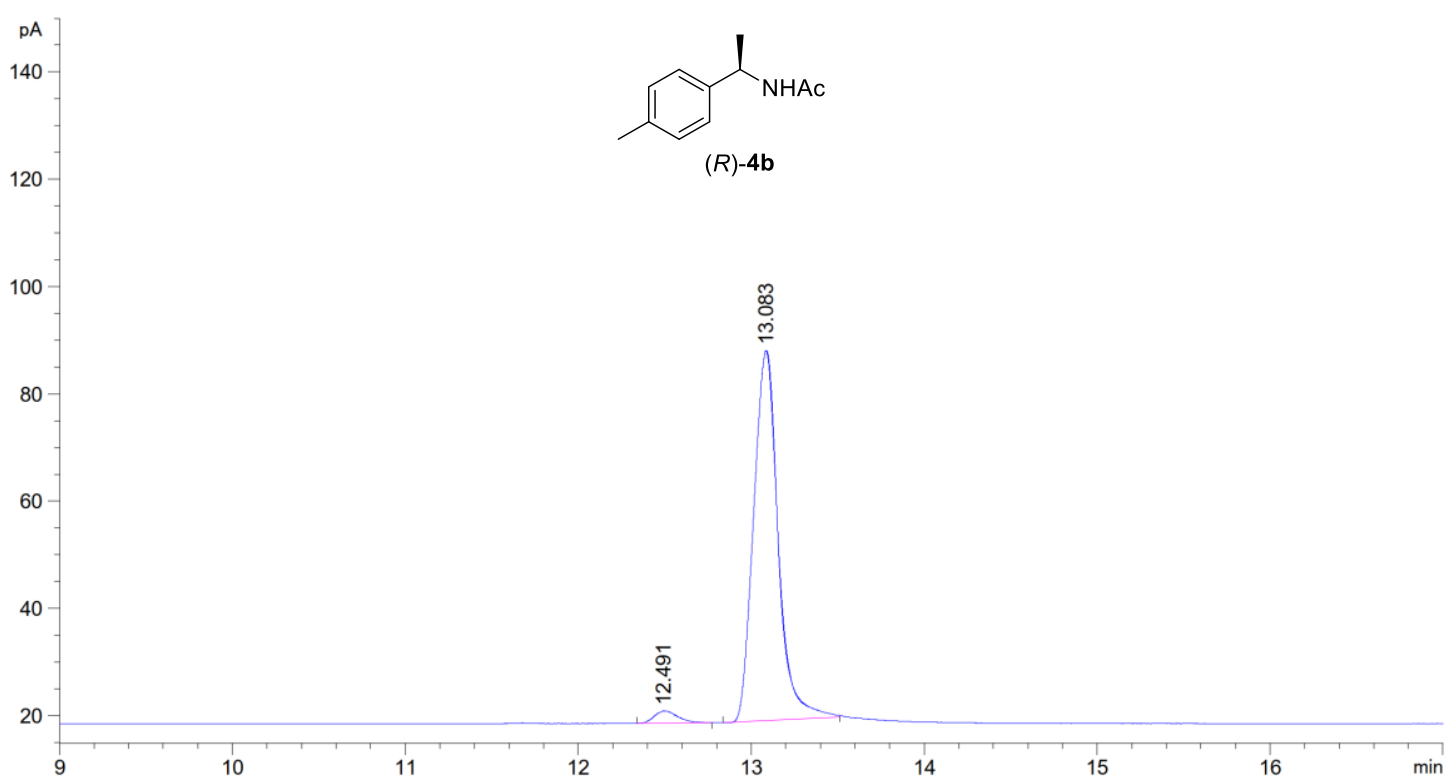

\begin{tabular}{|c|c|c|c|c|c|c|}
\hline $\begin{array}{r}\text { 峰 } \\
\#\end{array}$ & $\begin{array}{c}\text { 保留时间 } \\
\text { [min] }\end{array}$ & 类型 & $\begin{array}{l}\text { 峰宽 } \\
\text { [min] }\end{array}$ & $\begin{array}{c}\text { 峰面积 } \\
{\left[\mathrm{pA} *_{\mathrm{s}}\right]}\end{array}$ & $\begin{array}{l}\text { 峰高 } \\
{[\mathrm{pA}]}\end{array}$ & $\begin{array}{c}\text { 峰面积 } \\
\%\end{array}$ \\
\hline 1 & 12 & & 0.1553 & 21. 19101 & 2. 27484 & 3. 03631 \\
\hline 2 & 13. 083 & , & 0.1635 & 676.72760 & 68.98246 & 96.96369 \\
\hline
\end{tabular}




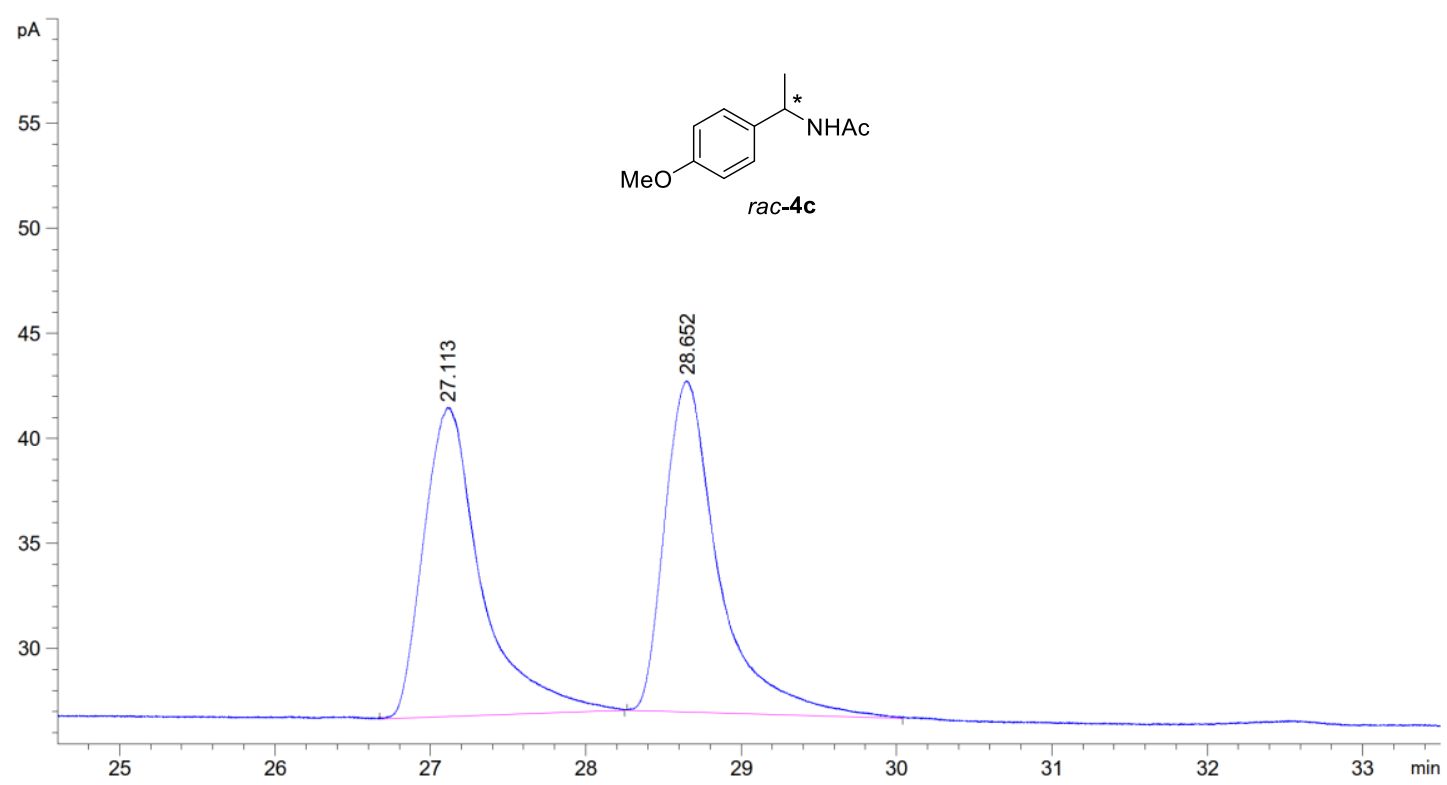

峰 保留时间类型峰宽峰面积峰高峰面积

\begin{tabular}{cccccc}
$\# \quad[\mathrm{~min}]$ & {$[\mathrm{min}]$} & {$[\mathrm{pA}$ s $]$} & {$[\mathrm{pA}]$} & $\%$ \\
\hline 1 & 27.113 BB & 0.3037 & 380.03418 & 14.71518 & 49.91941 \\
2 & $28.652 \mathrm{BB}$ & 0.2845 & 381.26123 & 15.76871 & 50.08059
\end{tabular}

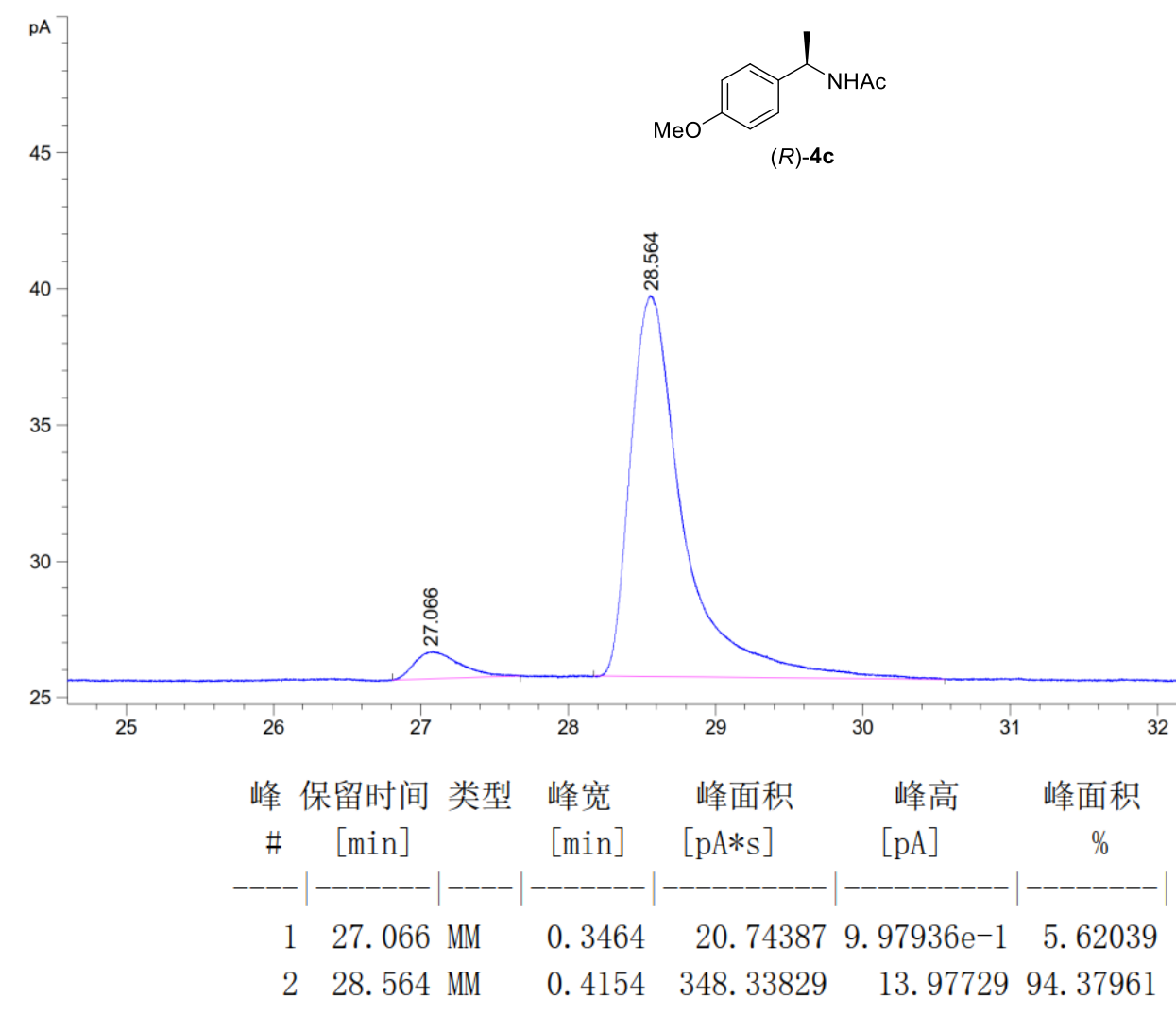




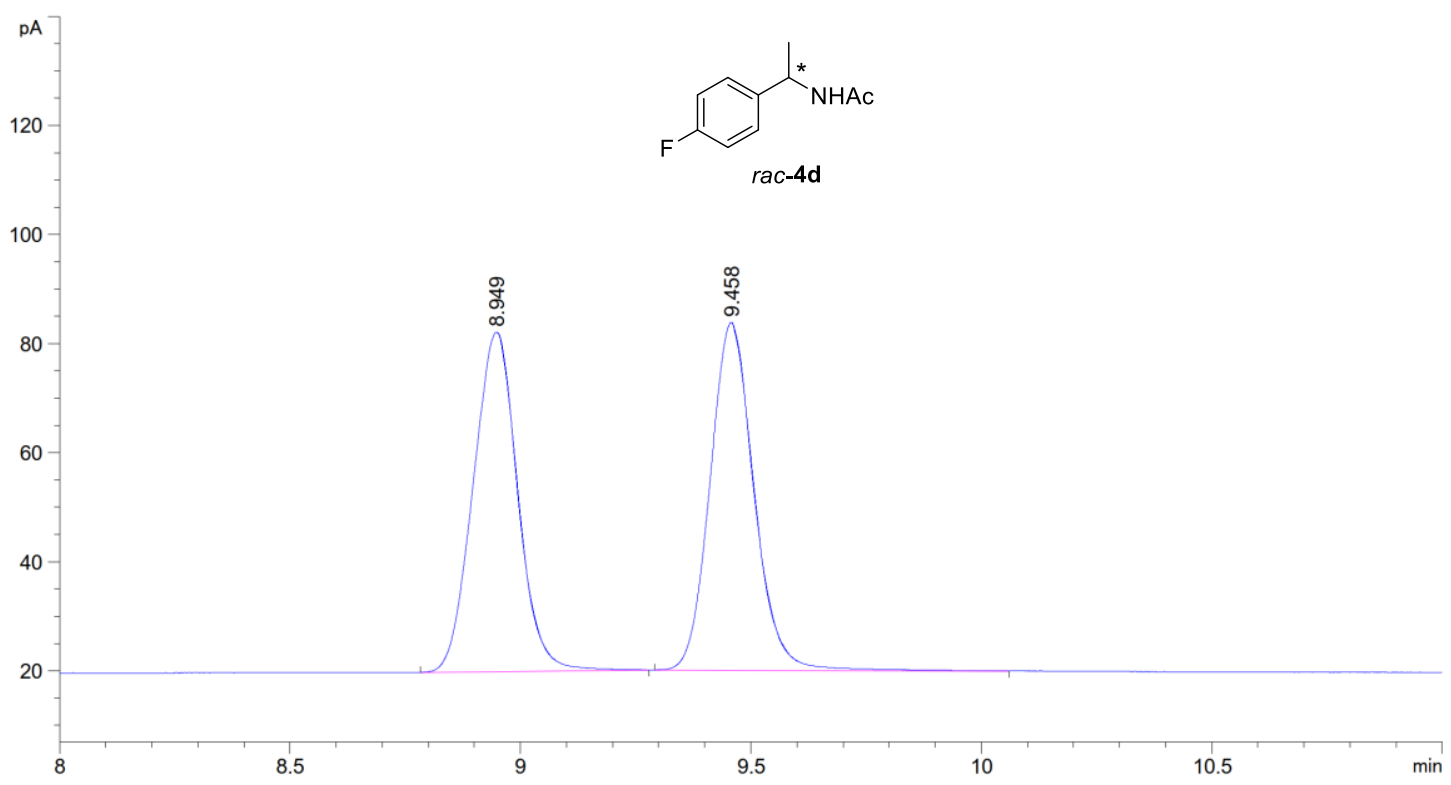

峰保留时间类型峰宽峰面积峰高峰面积

\begin{tabular}{cccccc}
$\#$ & {$[\mathrm{~min}]$} & {$[\mathrm{min}]$} & {$[\mathrm{pA}$ s $]$} & {$[\mathrm{pA}]$} & $\%$ \\
\hline 1 & 8.949 BB & 0.0966 & 417.31369 & 62.24757 & 49.73588 \\
2 & 9.458 BB & 0.0995 & 421.74588 & 63.78868 & 50.26412
\end{tabular}

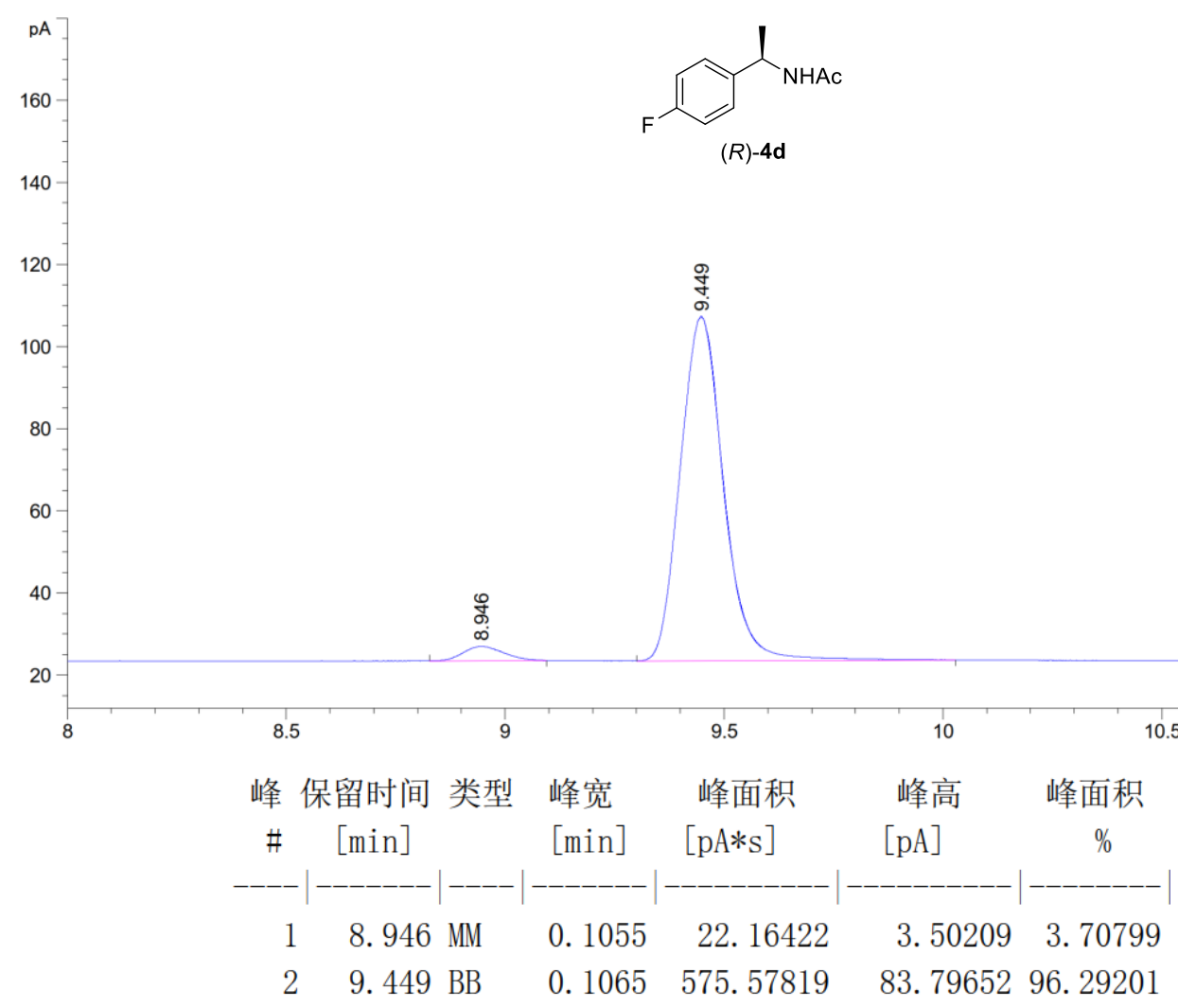



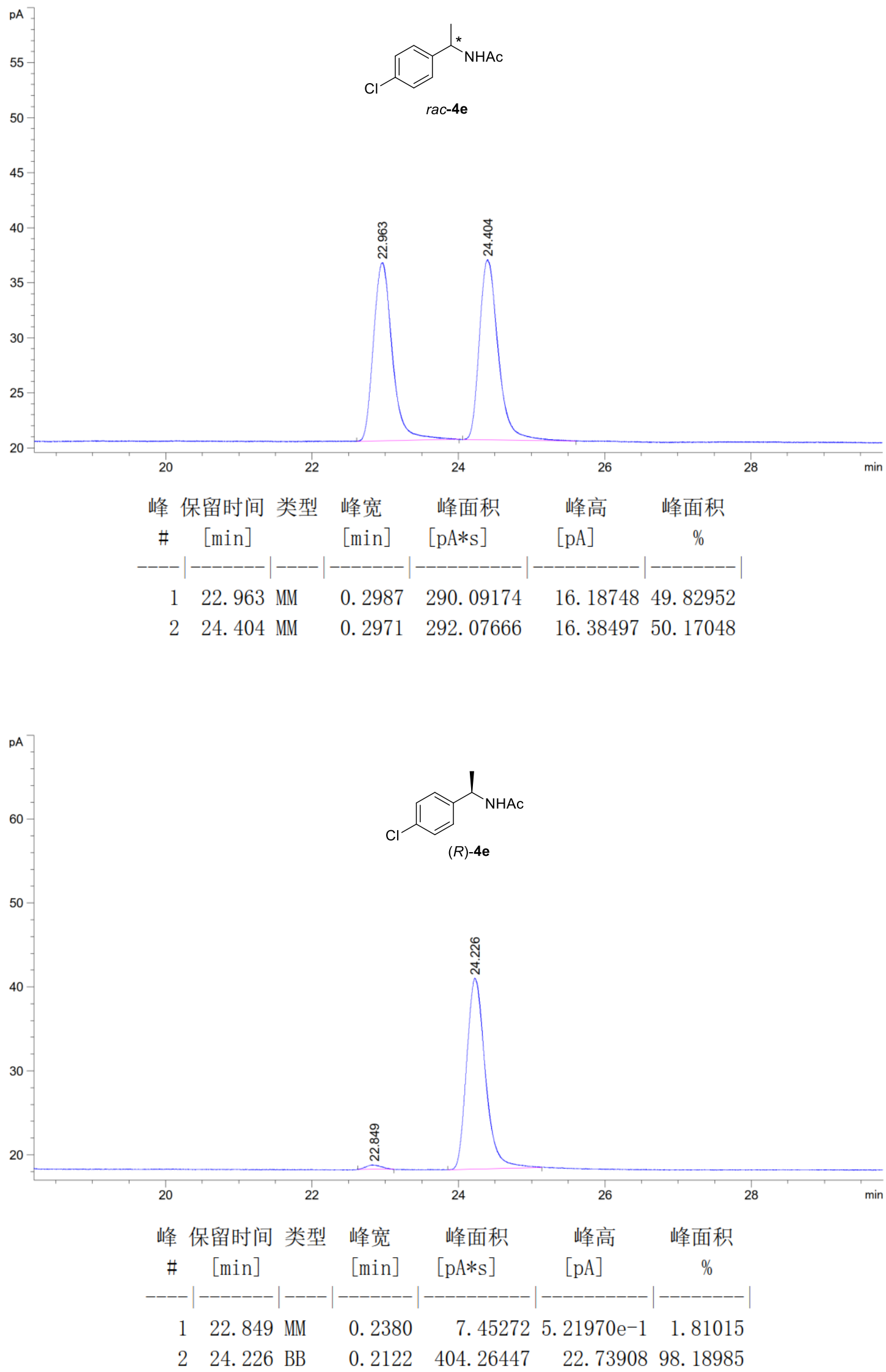


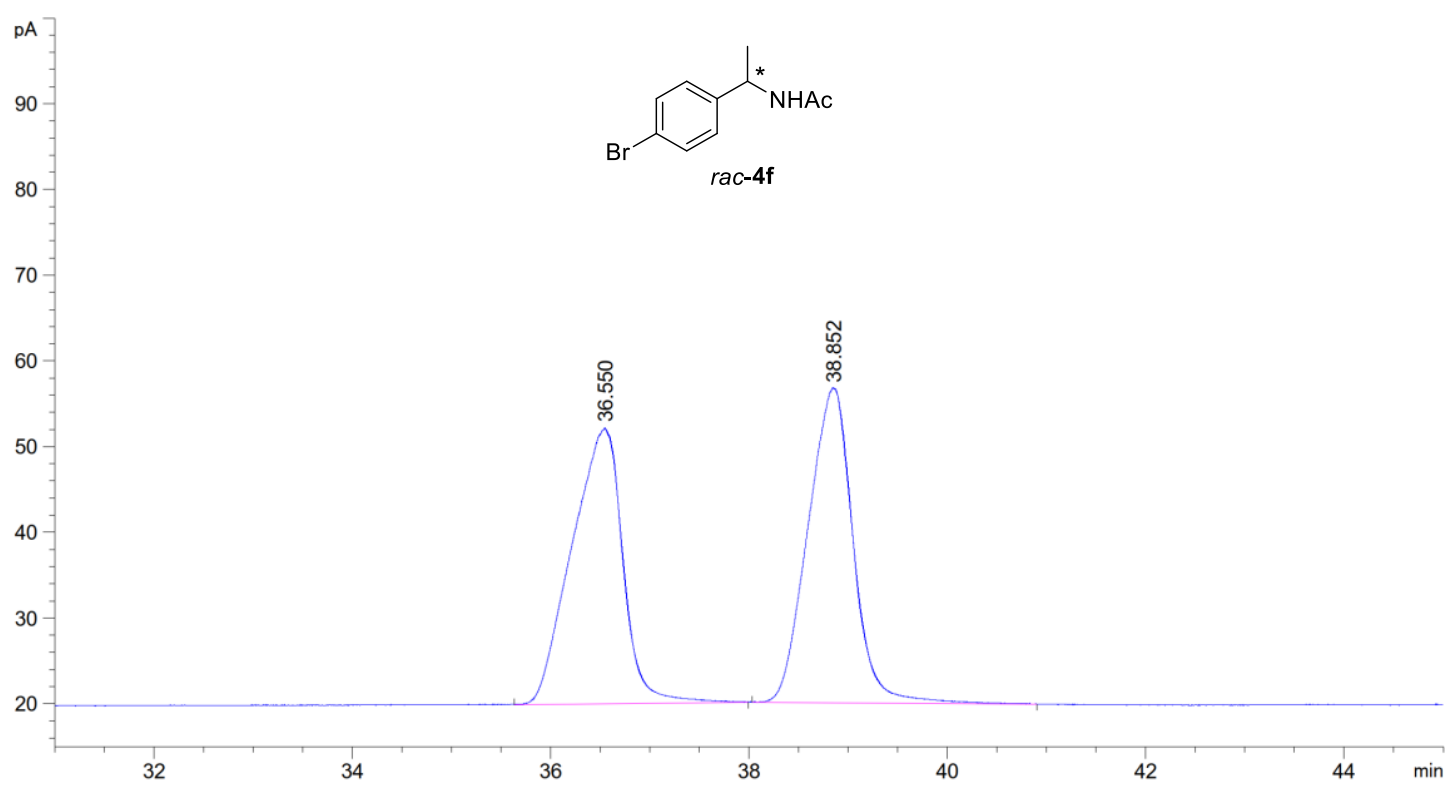

\begin{tabular}{|c|c|c|c|c|c|c|}
\hline $\begin{array}{r}\text { 峰 } \\
\text { \# }\end{array}$ & $\begin{array}{c}\text { 保留时间 } \\
\text { [min] }\end{array}$ & 类型 & $\begin{array}{l}\text { 峰宽 } \\
\text { [min] }\end{array}$ & $\begin{array}{l}\text { 峰面积 } \\
{\left[\mathrm{pA} *_{\mathrm{s}}\right]}\end{array}$ & $\begin{array}{l}\text { 峰高 } \\
{[\mathrm{pA}]}\end{array}$ & $\begin{array}{c}\text { 峰面积 } \\
\%\end{array}$ \\
\hline 1 & 36 . & & 0.4247 & 1147. 38599 & 32. 18442 & 49. 61627 \\
\hline 2 & 38.852 & & 0.3765 & 1165. 13379 & 36.73081 & 50.38373 \\
\hline
\end{tabular}

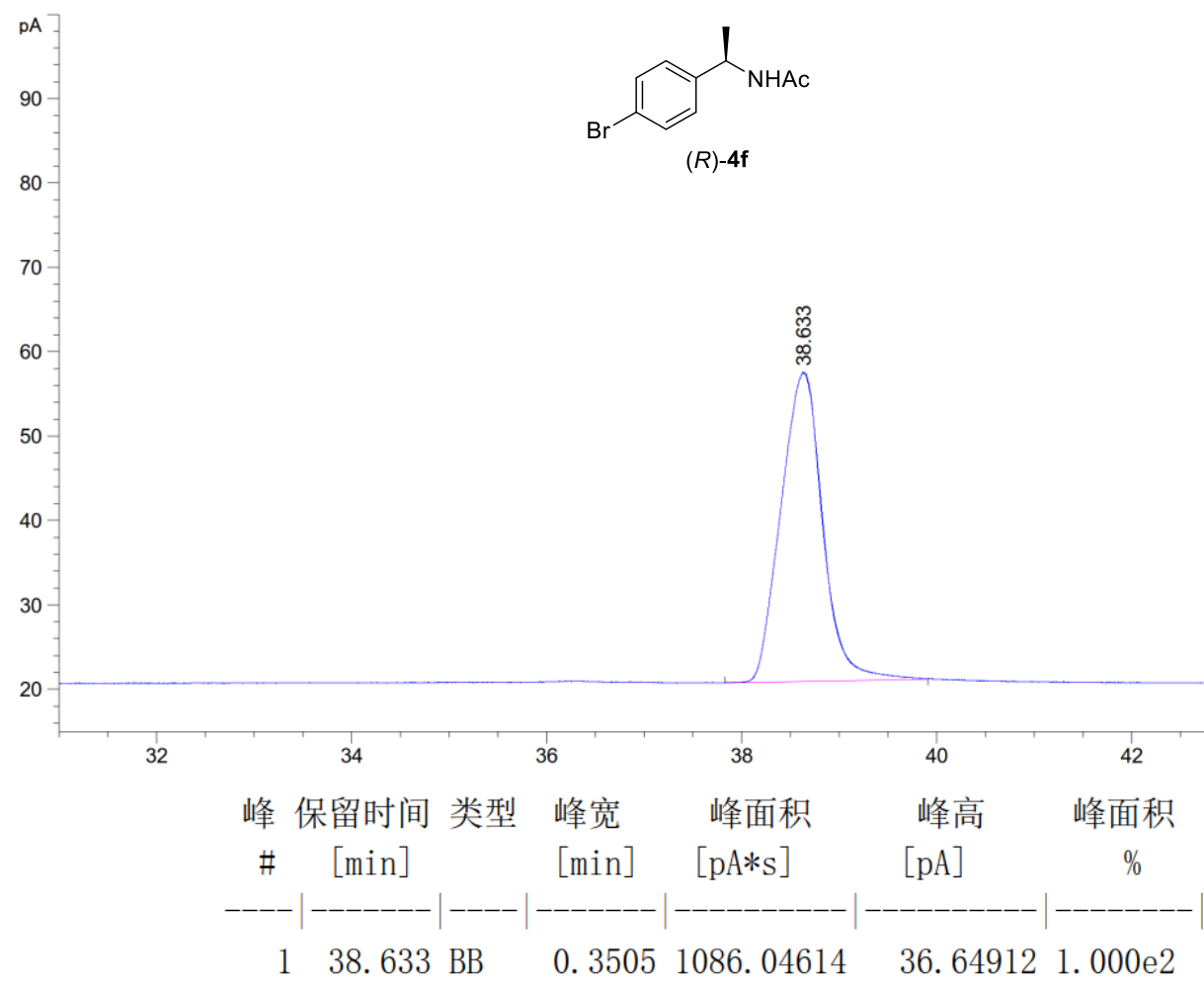




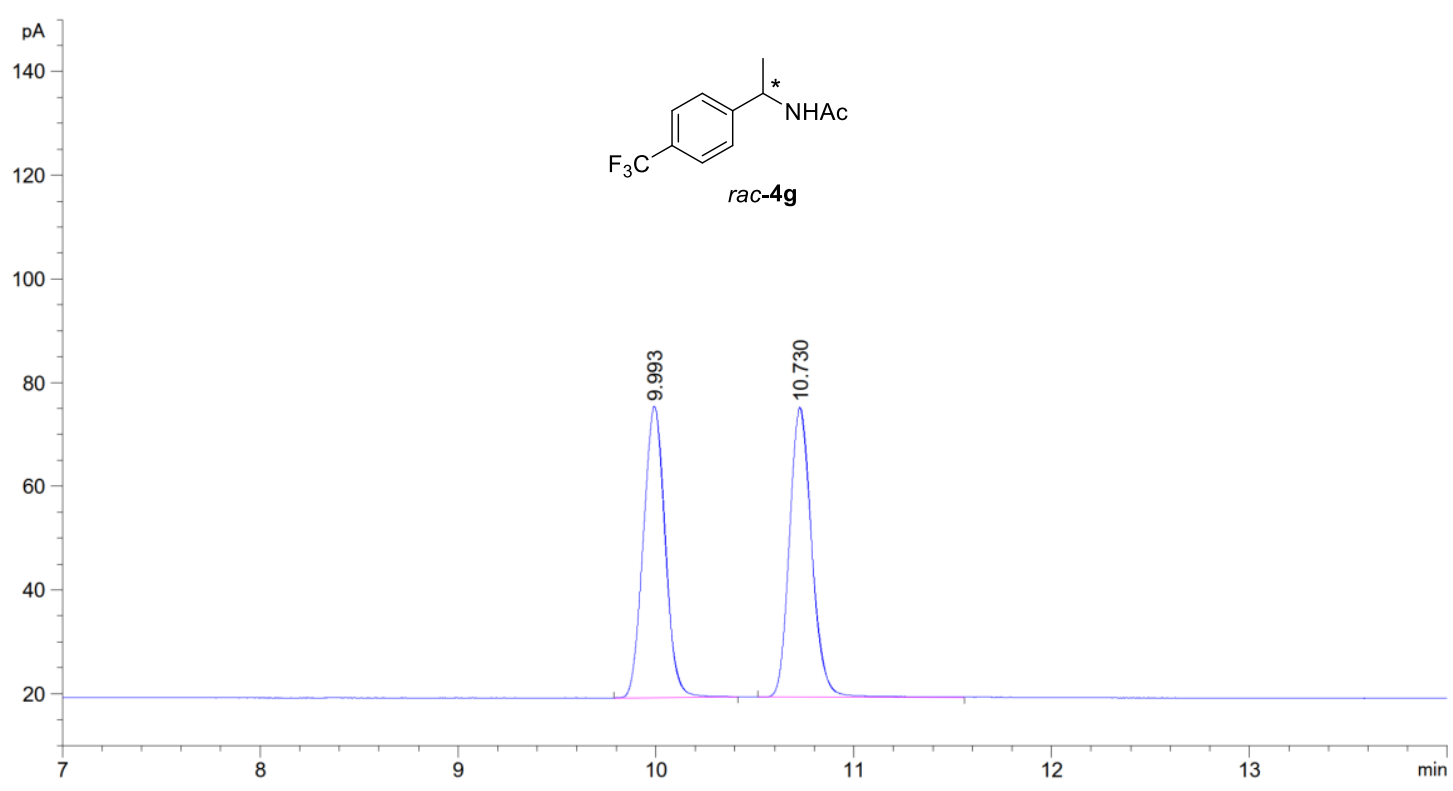

\begin{tabular}{|c|c|c|c|c|c|c|}
\hline 逢 & $\begin{array}{c}\text { 呆留时间 } \\
\text { [min] }\end{array}$ & 类型 & $\begin{array}{l}\text { 峰宽 } \\
\text { [min] }\end{array}$ & $\begin{array}{l}\text { 峰面积 } \\
{[\mathrm{pA} * \mathrm{~s}]}\end{array}$ & $\begin{array}{c}\text { 峰高 } \\
{[\mathrm{pA}]}\end{array}$ & $\begin{array}{c}\text { 峰面积 } \\
\%\end{array}$ \\
\hline & & & 0.1120 & 431. & 84 & 9 \\
\hline & 10.730 & & 0.1215 & 436. 66708 & 55.91251 & 50.30501 \\
\hline
\end{tabular}

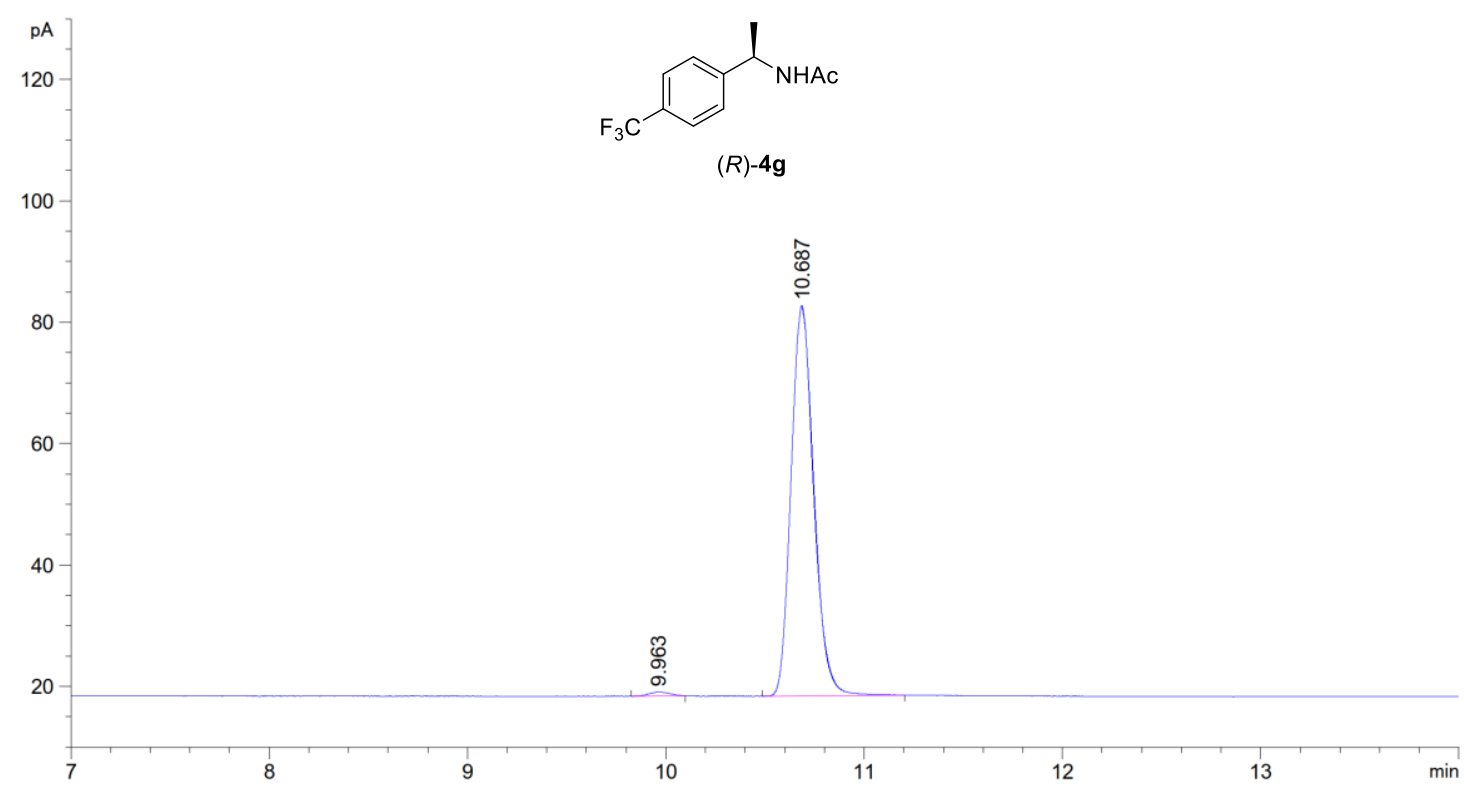

\begin{tabular}{|c|c|c|c|c|c|c|}
\hline 峰 & $\begin{array}{c}\text { 保留时间 } \\
\text { [min] }\end{array}$ & 类型 & $\begin{array}{l}\text { 峰宽 } \\
\text { [min] }\end{array}$ & $\begin{array}{c}\text { 峰面积 } \\
{\left[\mathrm{pA} *_{\mathrm{s}}\right]}\end{array}$ & $\begin{array}{c}\text { 峰高 } \\
{[\mathrm{pA}]}\end{array}$ & $\begin{array}{c}\text { 峰面积 } \\
\%\end{array}$ \\
\hline & & & 0.1 & 66 & $3 e^{-1}$ & 4179 \\
\hline & 10. 687 & & 0.1240 & 511.24744 & 64. 27779 & 99. 058 \\
\hline
\end{tabular}




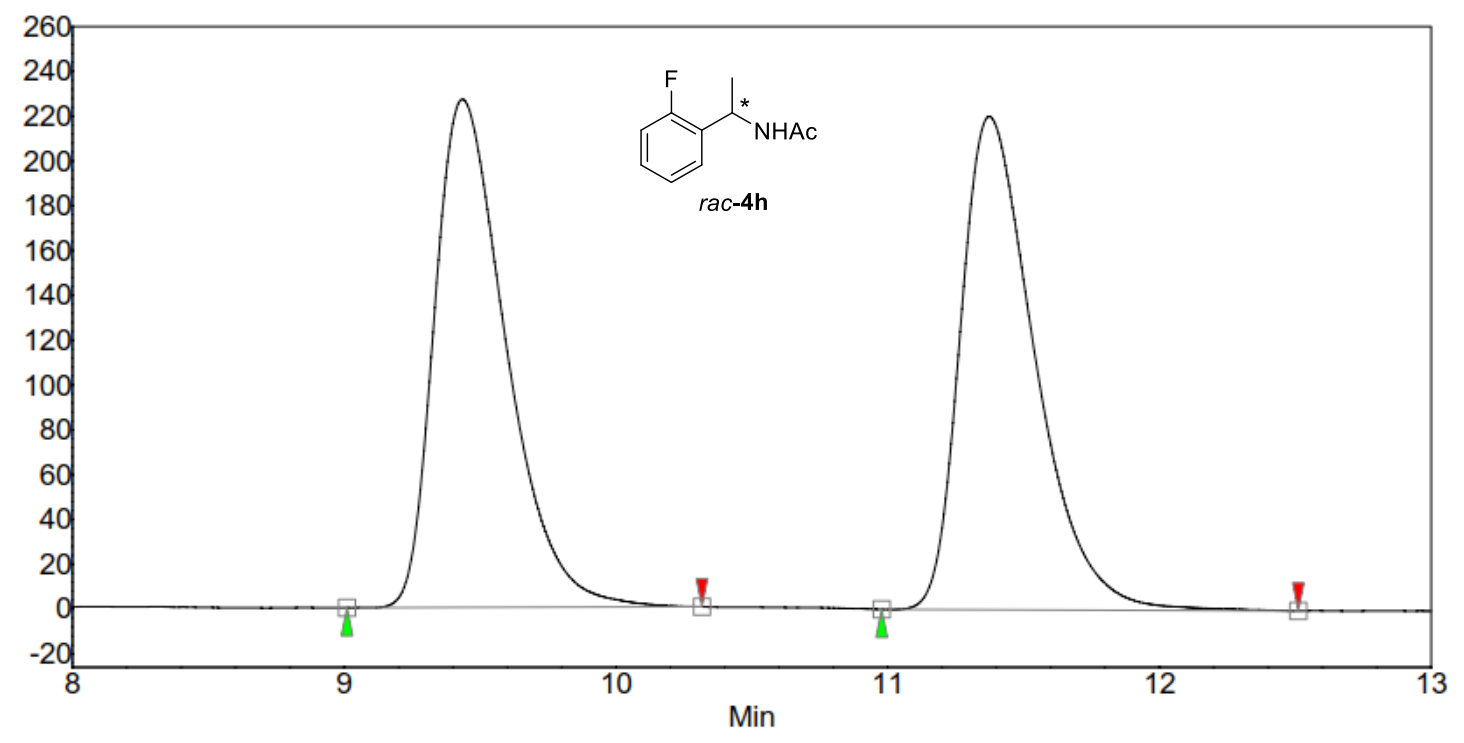

\begin{tabular}{|c|l|r|r|r|r|r|}
\hline Index & 文件名 & $\begin{array}{r}\text { 时间 } \\
\text { [Min] }\end{array}$ & $\begin{array}{r}\text { 数量 } \\
\text { [\% 面积] }\end{array}$ & $\begin{array}{r}\text { 高度 } \\
\text { [mAU] }\end{array}$ & $\begin{array}{r}\text { Area } \\
\text { [mAU.Min] }\end{array}$ & $\begin{array}{r}\text { Area \% } \\
{[\%]}\end{array}$ \\
\hline \hline 1 & 未知 & 9.43 & 49.79 & 226.9 & 69.4 & 49.793 \\
\hline 2 & 未知 & 11.37 & 50.21 & 220.3 & 70.0 & 50.207 \\
\hline & & & & & & \\
\hline Total & & & 100.00 & 447.1 & 139.4 & 100.000 \\
\hline
\end{tabular}

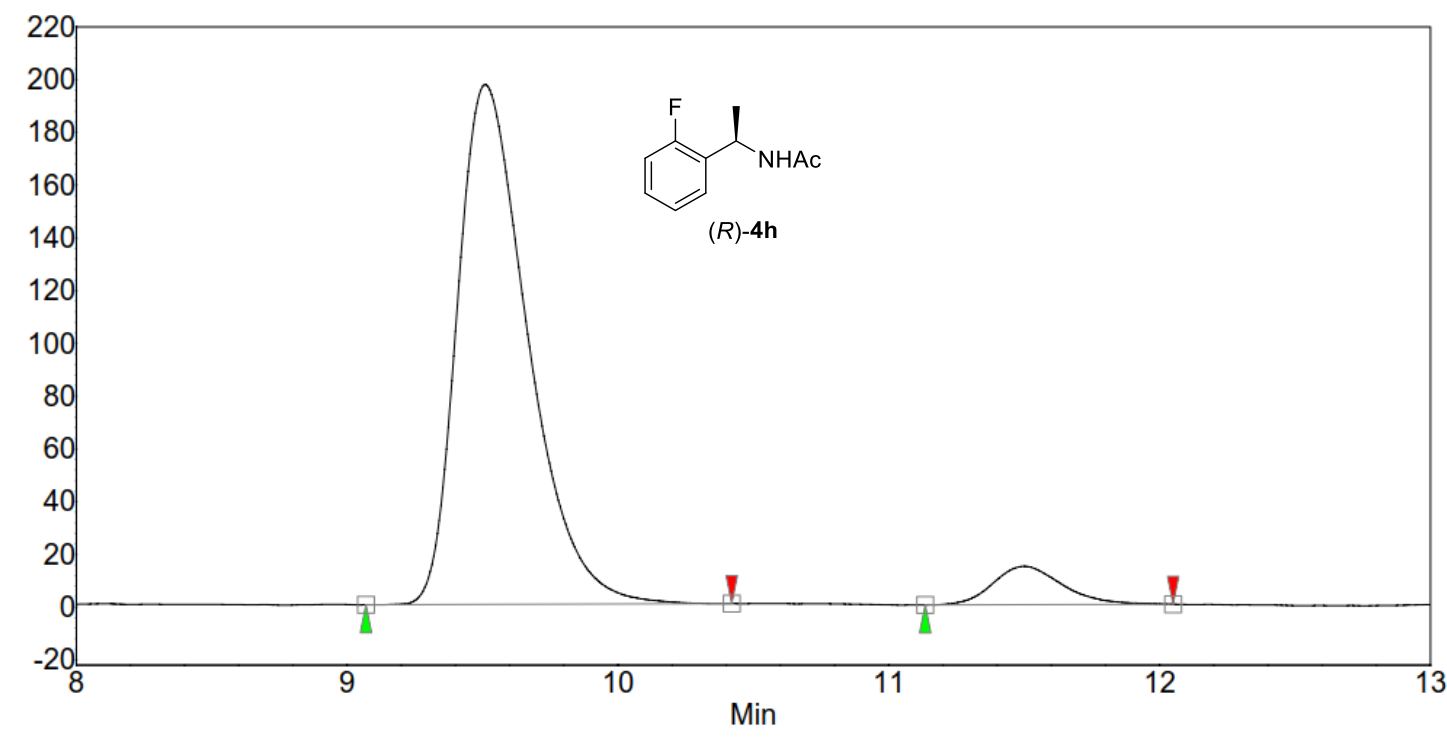

\begin{tabular}{|c|l|r|r|r|r|r|}
\hline Index & 文件名 & $\begin{array}{r}\text { 时间 } \\
\text { [Min] }\end{array}$ & $\begin{array}{r}\text { 数量 } \\
\text { [\% 面积] }\end{array}$ & $\begin{array}{r}\text { 高度 } \\
\text { [mAU] }\end{array}$ & $\begin{array}{r}\text { Area } \\
\text { [mAU.Min] }\end{array}$ & $\begin{array}{r}\text { Area \% } \\
{[\%]}\end{array}$ \\
\hline \hline 1 & 未知 & 9.51 & 93.25 & 197.1 & 59.4 & 93.245 \\
\hline 2 & 未知 & 11.50 & 6.75 & 14.5 & 4.3 & 6.755 \\
\hline & & & & & & \\
\hline Total & & & 100.00 & 211.6 & 63.7 & 100.000 \\
\hline
\end{tabular}



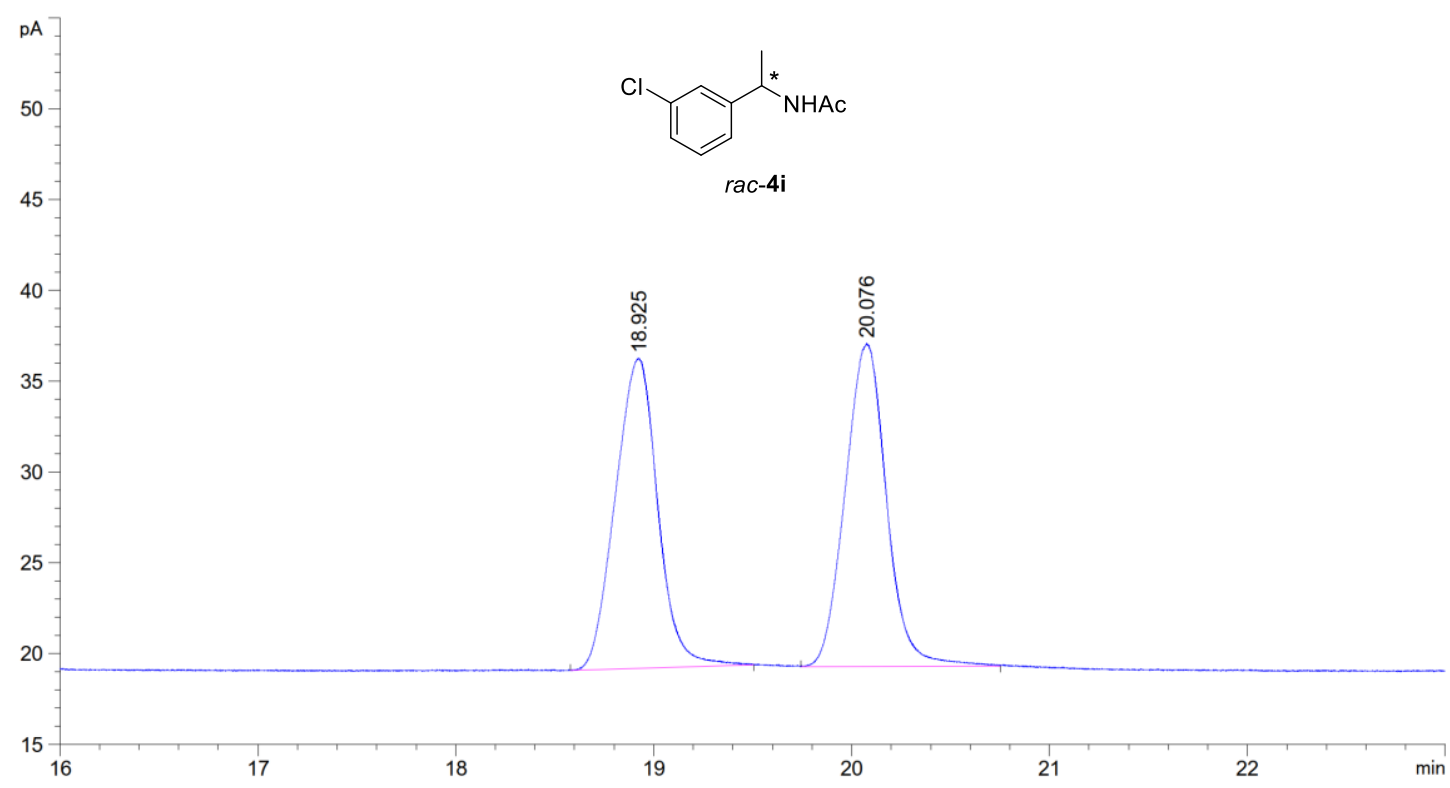

峰保留时间 类型峰宽峰面积峰高峰面积

\begin{tabular}{cccccc}
$\#$ & {$[\mathrm{~min}]$} & {$[\mathrm{min}]$} & {$[\mathrm{pA}$ s $]$} & {$[\mathrm{pA}]$} & $\%$ \\
\hline 1 & $18.925 \mathrm{BB}$ & 0.1787 & 254.51295 & 17.07800 & 49.72259 \\
2 & 20.076 BB & 0.1707 & 257.35287 & 17.75992 & 50.27741
\end{tabular}

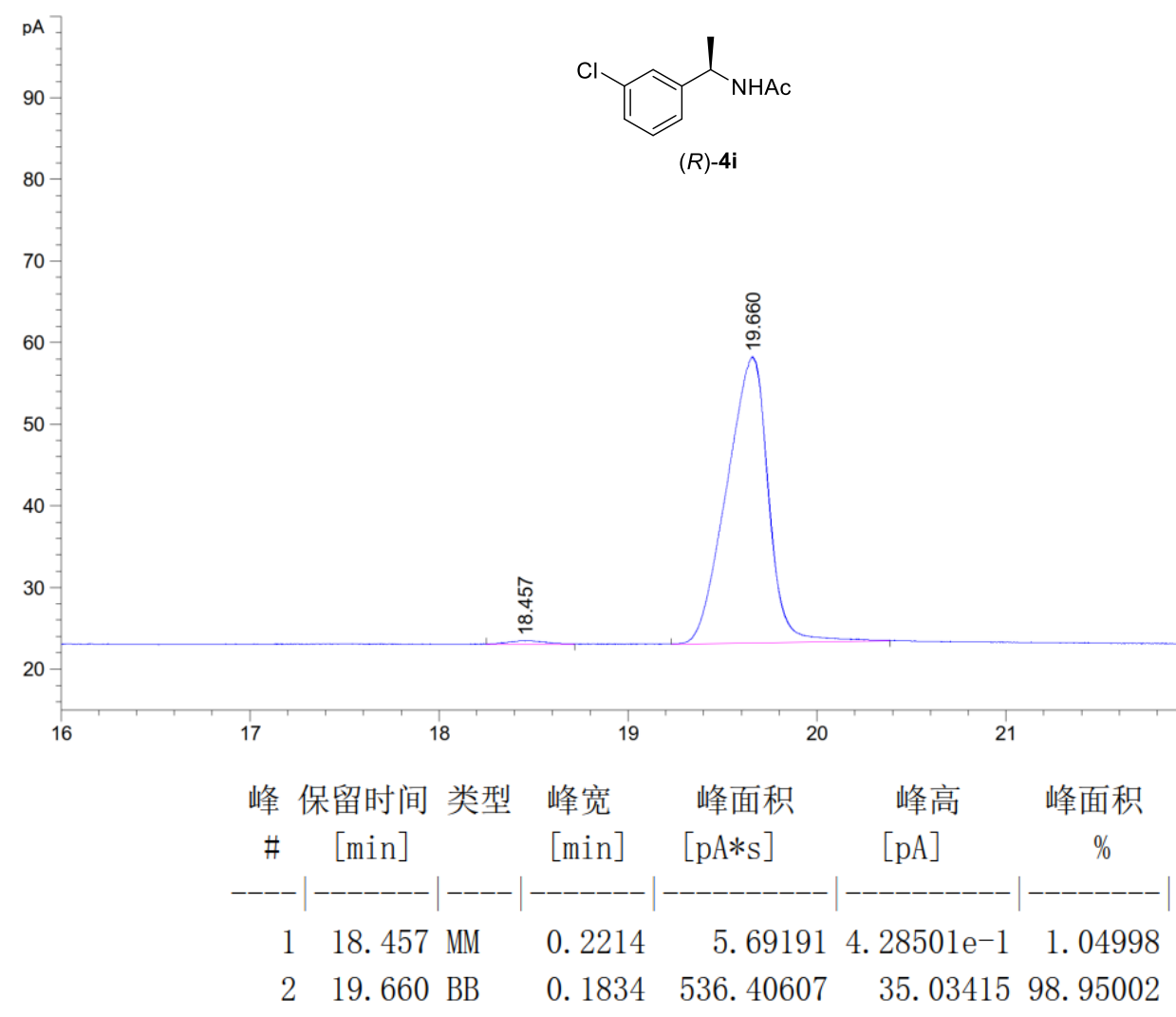



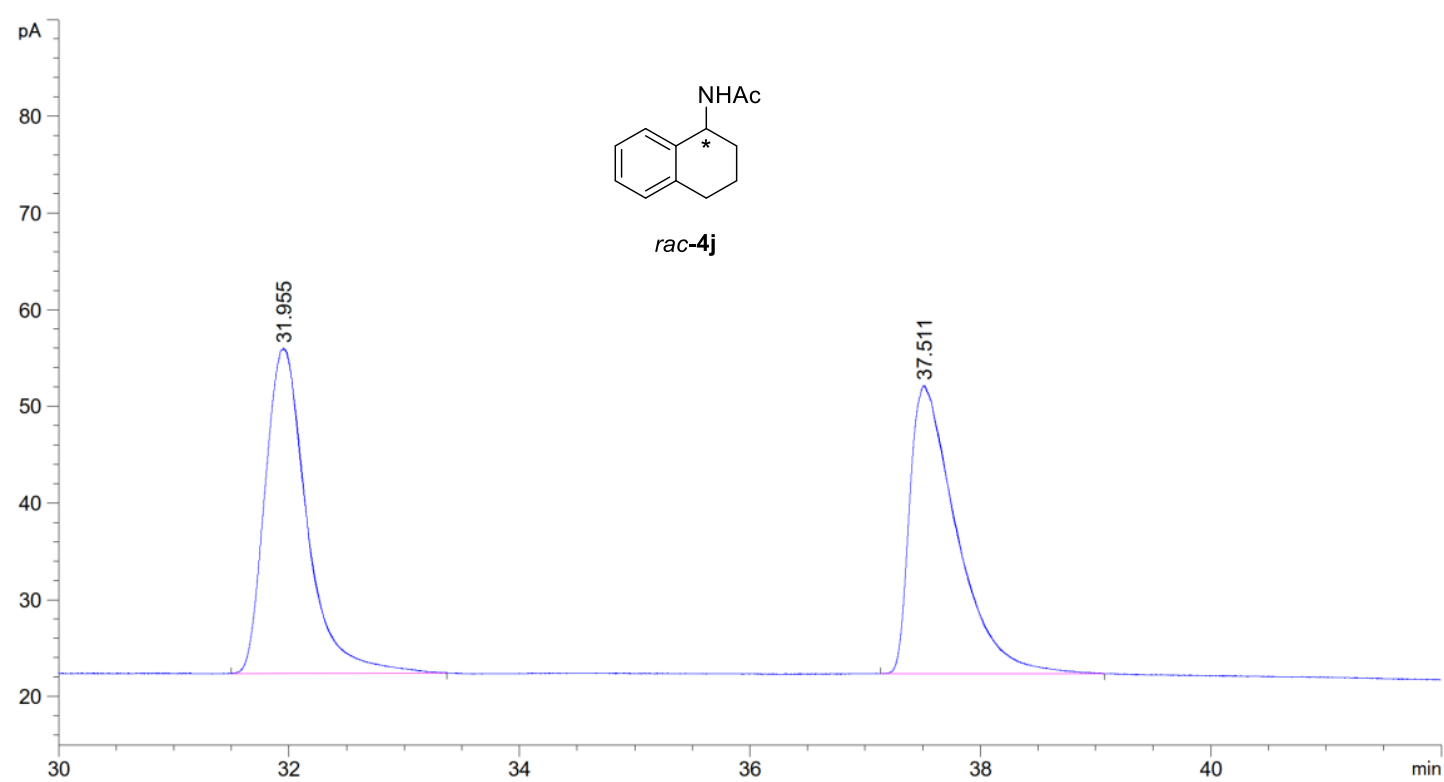

峰 保留时间 类型 峰宽 峰面积 峰高 峰面积

$\begin{array}{cccccc}\# & {[\mathrm{~min}]} & {[\mathrm{min}]} & {[\mathrm{pA} * \mathrm{~s}]} & {[\mathrm{pA}]} & \% \\ -1 & 31.955 \mathrm{BB} & 0.2963 & 849.67145 & 33.63360 & 50.12179 \\ 2 & 37.511 \text { BB } & 0.3342 & 845.54224 & 29.80530 & 49.87821\end{array}$
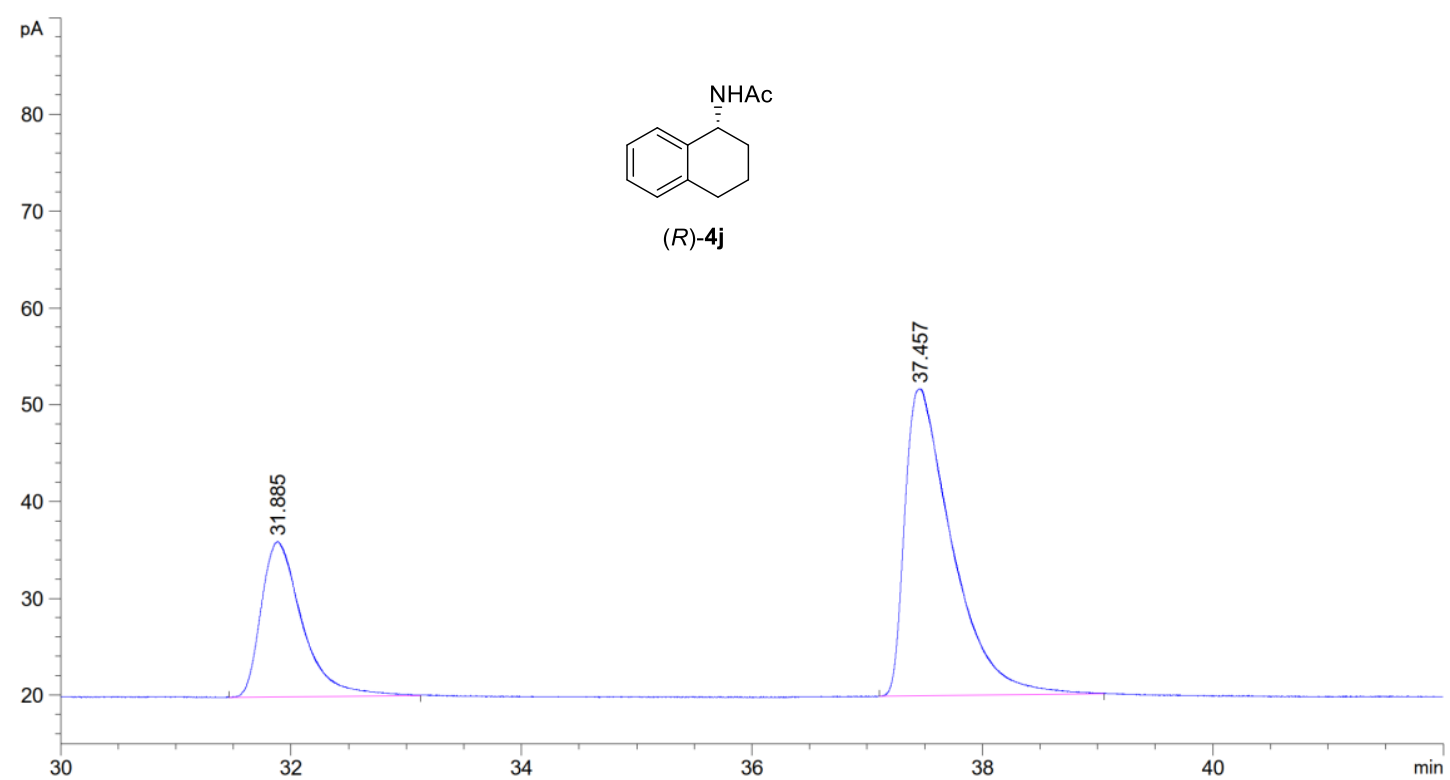

峰 保留时间 类型 峰宽 峰面积 峰高 峰面积

\begin{tabular}{|c|c|c|c|c|c|}
\hline \# & [min] & {$[\mathrm{min}]$} & {$[\mathrm{pA} * \mathrm{~s}]$} & {$[\mathrm{pA}]$} & $\%$ \\
\hline & 85 & 0.2922 & 397.68707 & 16. 05609 & 30.526 \\
\hline & 37. $457 \mathrm{BB}$ & 0.3366 & 905.07318 & 31.74497 & 69. 47350 \\
\hline
\end{tabular}


Asymmetric hydrogenation of enamides using $(S, S)-\mathrm{L} 3$

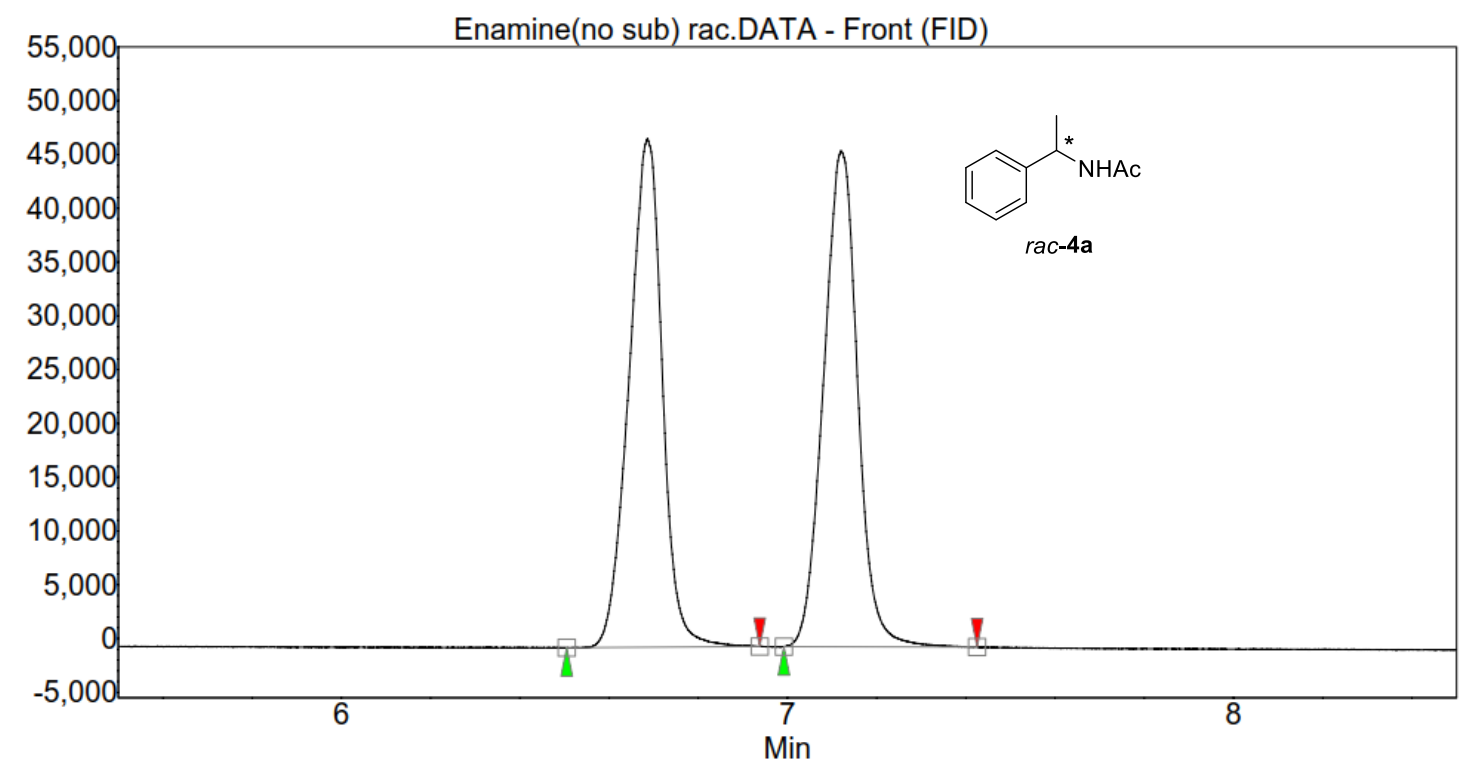

Peak results :

\begin{tabular}{|c|l|r|r|r|r|r|}
\hline Index & 文件名 & $\begin{array}{r}\text { 时间 } \\
\text { [Min] }\end{array}$ & $\begin{array}{r}\text { 数量 } \\
\text { [\% 面积] }\end{array}$ & $\begin{array}{r}\text { 高度 } \\
\text { [磺] }\end{array}$ & $\begin{array}{r}\text { Area } \\
\text { [磺.Min] }\end{array}$ & $\begin{array}{r}\text { Area \% } \\
\text { [\%] }\end{array}$ \\
\hline \hline 1 & 未知 & 6.69 & 49.99 & 47261.4 & 4071.6 & 49.987 \\
\hline 2 & 未知 & 7.12 & 50.01 & 46102.1 & 4073.6 & 50.013 \\
\hline & & & & & & \\
\hline Total & & & 100.00 & 93363.4 & 8145.2 & 100.000 \\
\hline
\end{tabular}

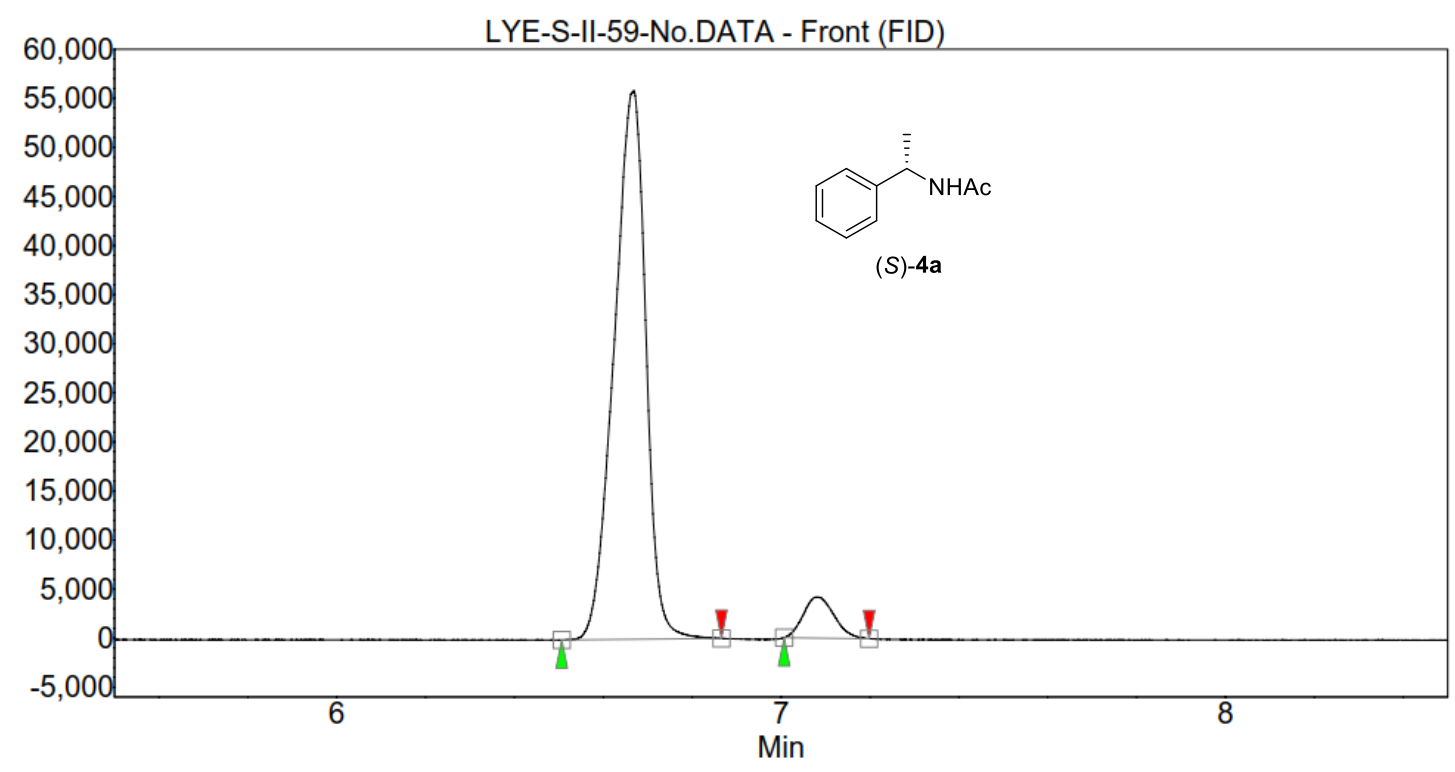

Peak results :

\begin{tabular}{|c|l|r|r|r|r|r|}
\hline Index & 文件名 & $\begin{array}{r}\text { 时间 } \\
\text { [Min] }\end{array}$ & $\begin{array}{r}\text { 数量 } \\
\text { 面积] }\end{array}$ & $\begin{array}{r}\text { 高度 } \\
\text { [磺] }\end{array}$ & $\begin{array}{r}\text { Area } \\
\text { [礧.Min] }\end{array}$ & $\begin{array}{r}\text { Area \% } \\
\text { [\%] }\end{array}$ \\
\hline \hline 2 & 未知 & 6.67 & 93.23 & 55896.1 & 4614.2 & 93.234 \\
\hline 1 & 未知 & 7.08 & 6.77 & 4159.8 & 334.9 & 6.766 \\
\hline & & & & & & \\
\hline Total & & & 100.00 & 60055.9 & 4949.0 & 100.000 \\
\hline
\end{tabular}



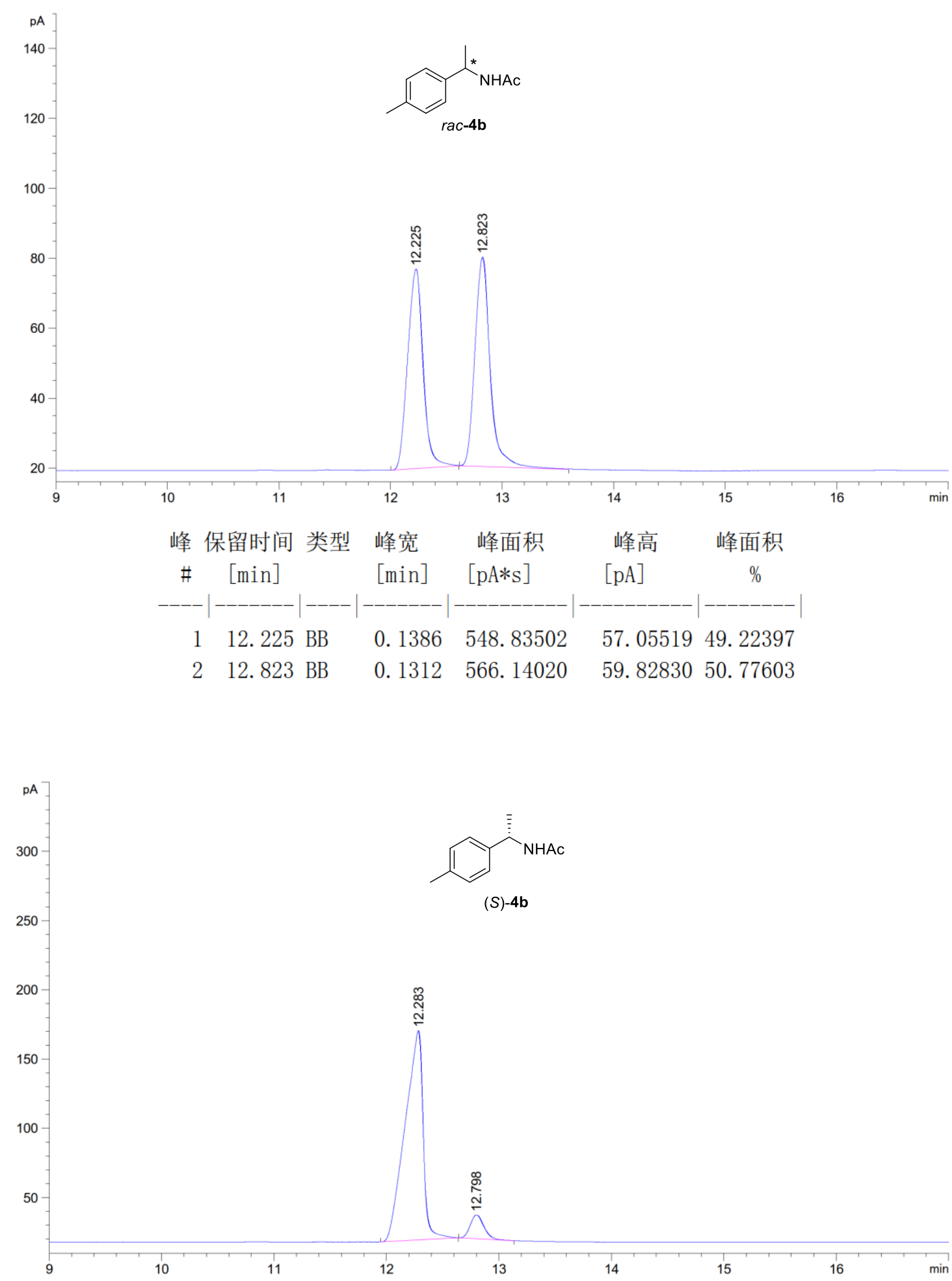

峰 保留时间 类型 峰宽 峰面积峰高峰面积

\begin{tabular}{cccccc}
$\#$ & {$[\mathrm{~min}]$} & {$[\mathrm{min}]$} & {$[\mathrm{pA} * \mathrm{~s}]$} & \multicolumn{1}{c}{$[\mathrm{pA}]$} & $\%$ \\
\hline 1 & $12.283 \mathrm{BB}$ & 0.1475 & 1692.51611 & 151.10838 & 92.17685 \\
2 & 12.798 BB & 0.1211 & 143.64569 & 17.11806 & 7.82315
\end{tabular}




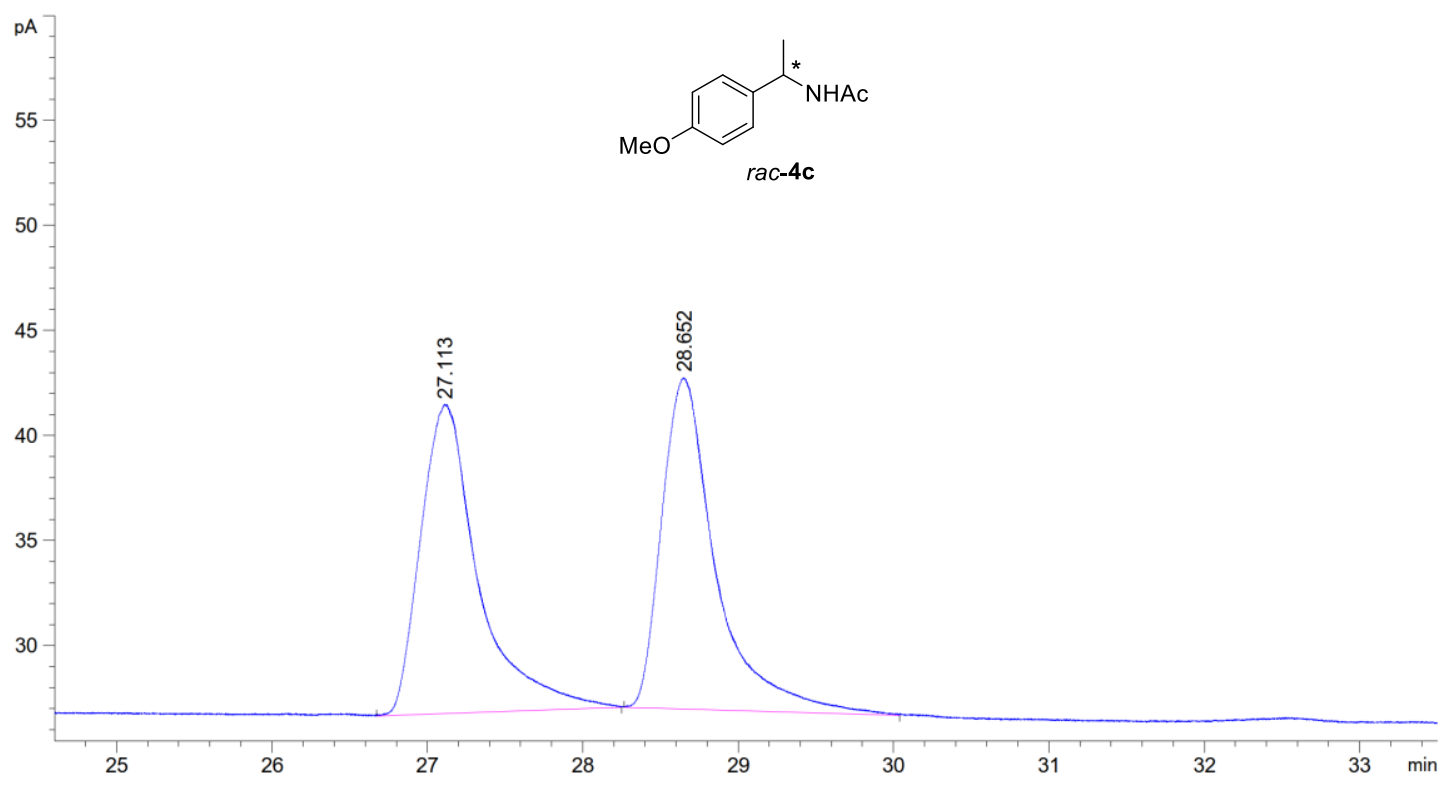

峰 保留时间类型峰宽峰面积峰高峰面积

\begin{tabular}{cccccc}
$\#$ & {$[\mathrm{~min}]$} & {$[\mathrm{min}]$} & {$[\mathrm{pA} * \mathrm{~s}]$} & {$[\mathrm{pA}]$} & $\%$ \\
\hline 1 & 27.113 BB & 0.3037 & 380.03418 & 14.71518 & 49.91941 \\
2 & $28.652 \mathrm{BB}$ & 0.2845 & 381.26123 & 15.76871 & 50.08059
\end{tabular}

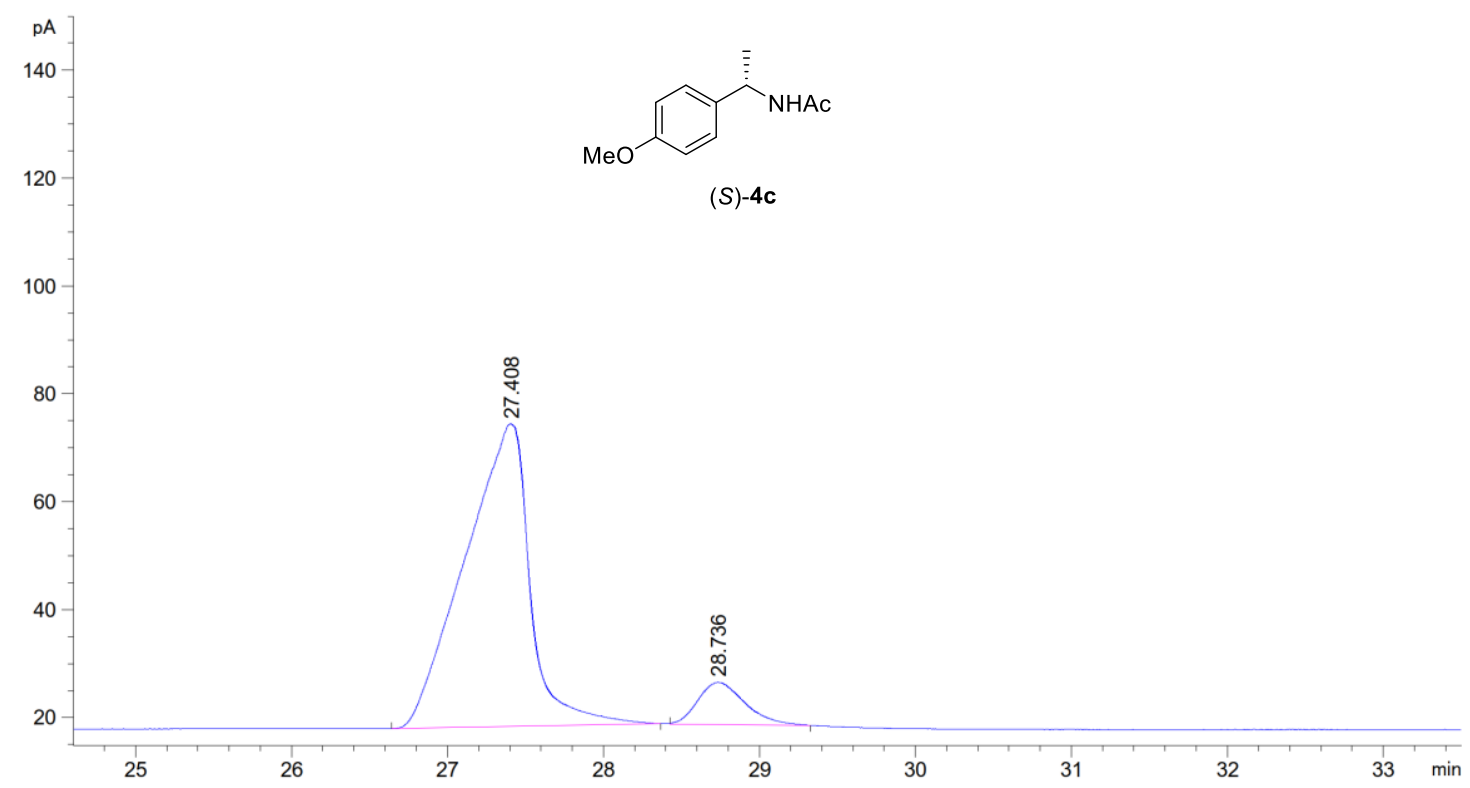

峰 保留时间类型峰宽峰面积峰高峰面积

\begin{tabular}{cccccc}
$\#$ & {$[\mathrm{~min}]$} & {$[\mathrm{min}]$} & {$[\mathrm{pA} * \mathrm{~s}]$} & \multicolumn{1}{c}{$[\mathrm{pA}]$} & \multicolumn{1}{c}{$\%$} \\
\hline 1 & $27.408 \mathrm{BB}$ & 0.3459 & 1631.07874 & 56.05799 & 90.87191 \\
2 & $28.736 \mathrm{MM}$ & 0.3473 & 163.84192 & 7.86259 & 9.12809
\end{tabular}



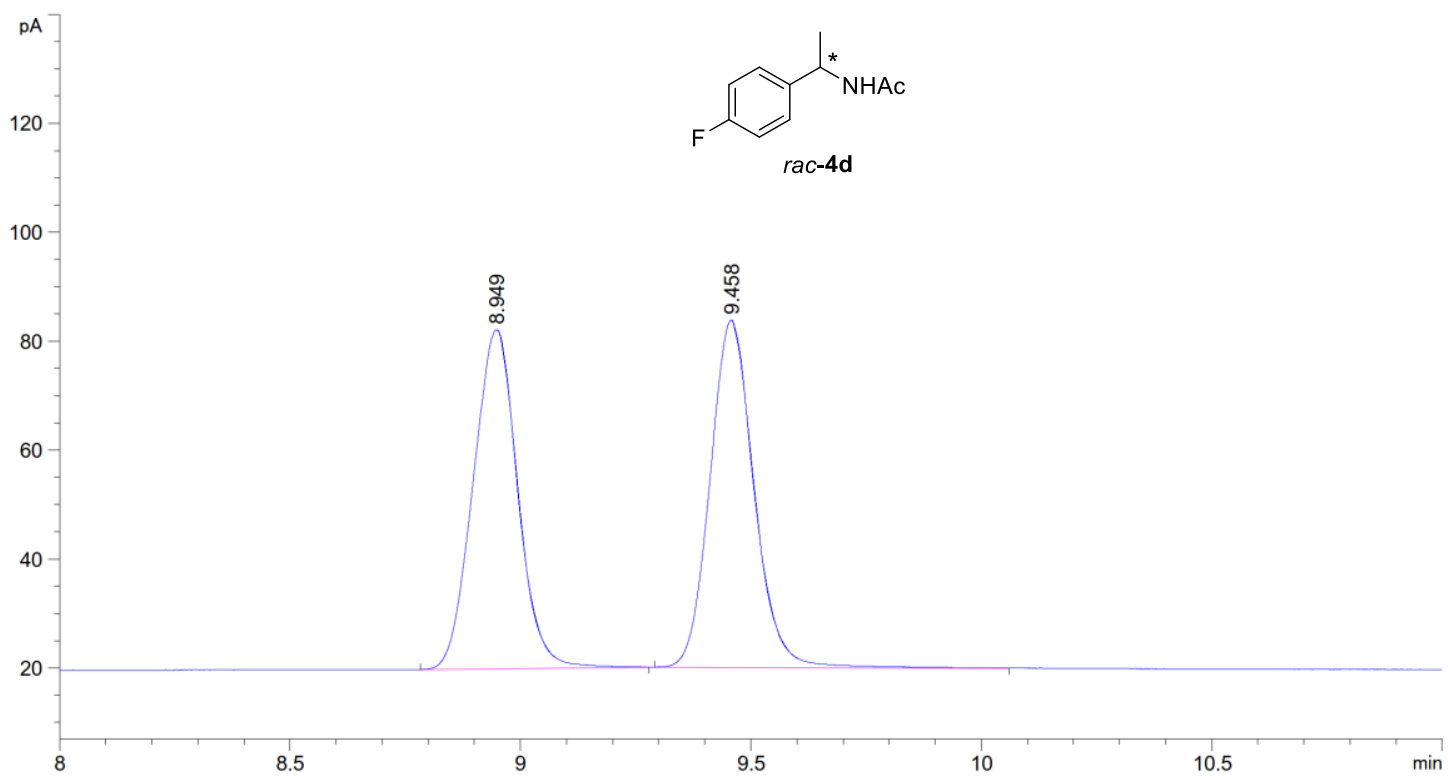

峰 保留时间 类型 峰宽 峰面积峰高峰面积

$\begin{array}{cccccc}\# & {[\mathrm{~min}]} & {[\mathrm{min}]} & {[\mathrm{pA} * \mathrm{~s}]} & {[\mathrm{pA}]} & \% \\ 1 & 8.949 \text { BB } & 0.0966 & 417.31369 & 62.24757 & 49.73588 \\ 2 & 9.458 \text { BB } & 0.0995 & 421.74588 & 63.78868 & 50.26412\end{array}$

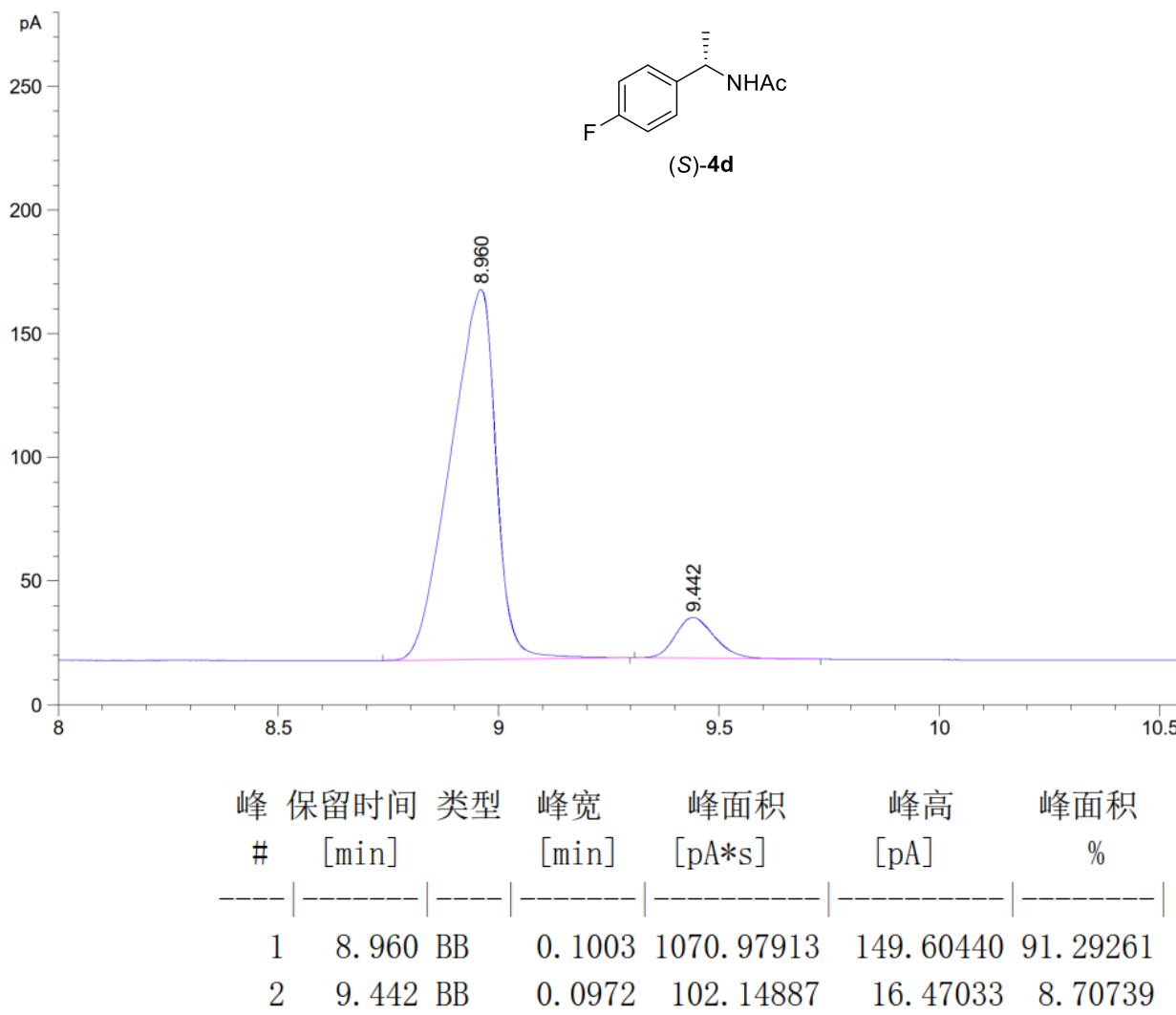



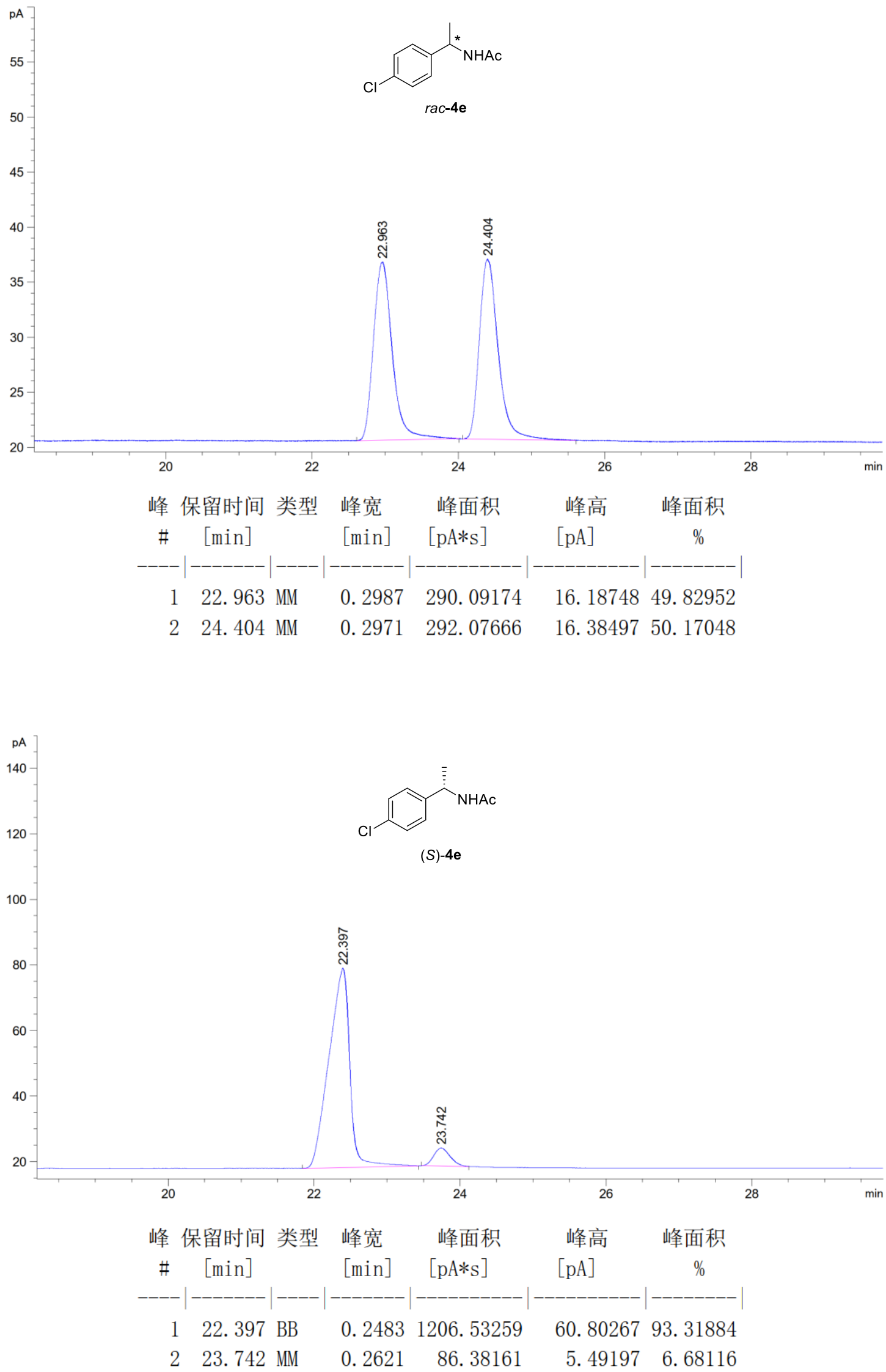


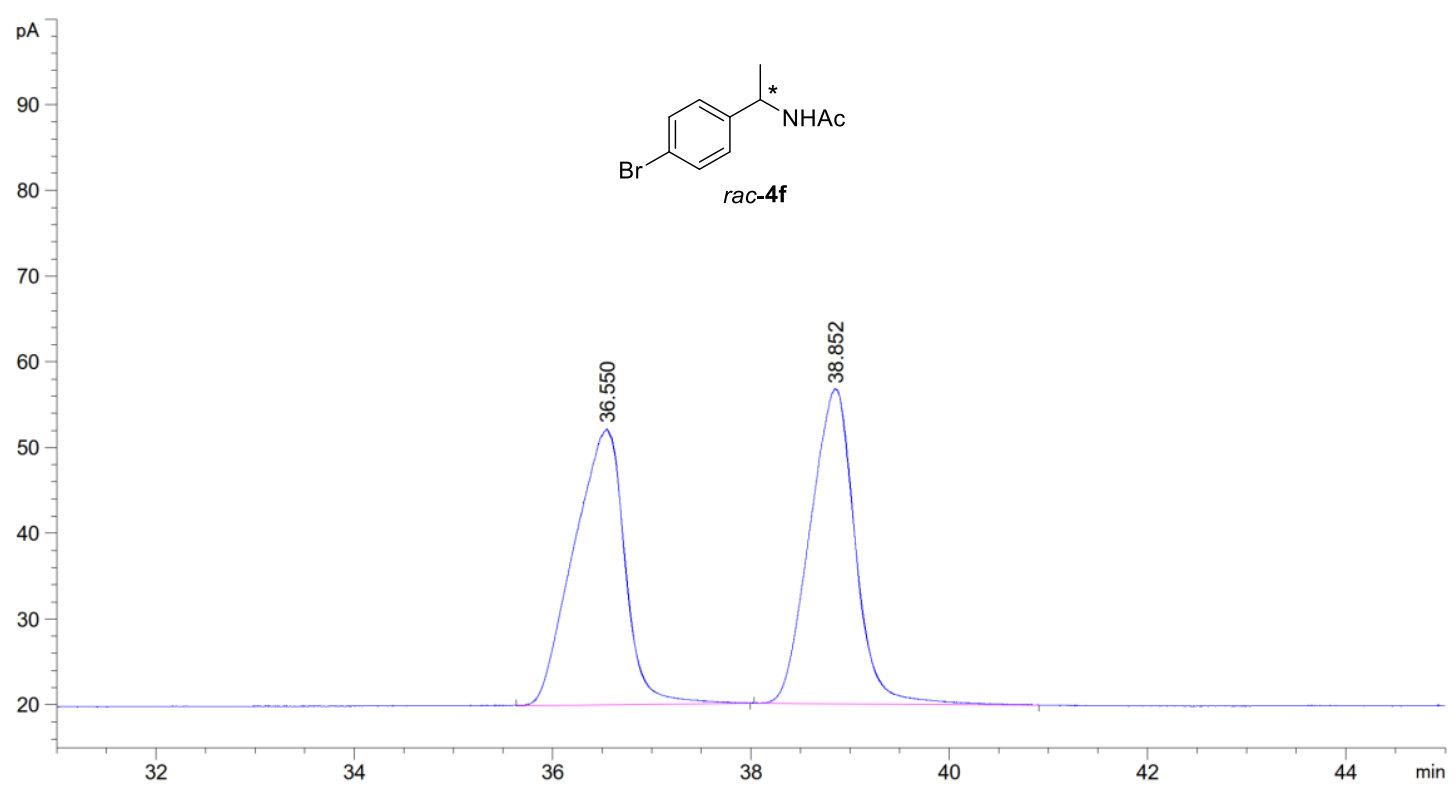

峰保留时间 类型 峰宽 峰面积 峰高 峰面积

\begin{tabular}{|c|c|c|c|c|c|}
\hline & {$[\mathrm{min}]$} & {$[\min ]$} & {$[\mathrm{pA} * \mathrm{~s}]$} & {$[\mathrm{pA}]$} & $\%$ \\
\hline 1 & 36. & 0.4247 & 1147 & 32. 18442 & 49. 61627 \\
\hline & 38. $852 \mathrm{BB}$ & 0.3765 & 1165. 13379 & 36.73081 & 50. 38373 \\
\hline
\end{tabular}

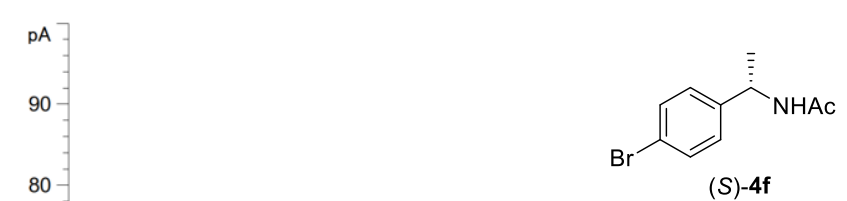

$(S)-4 f$

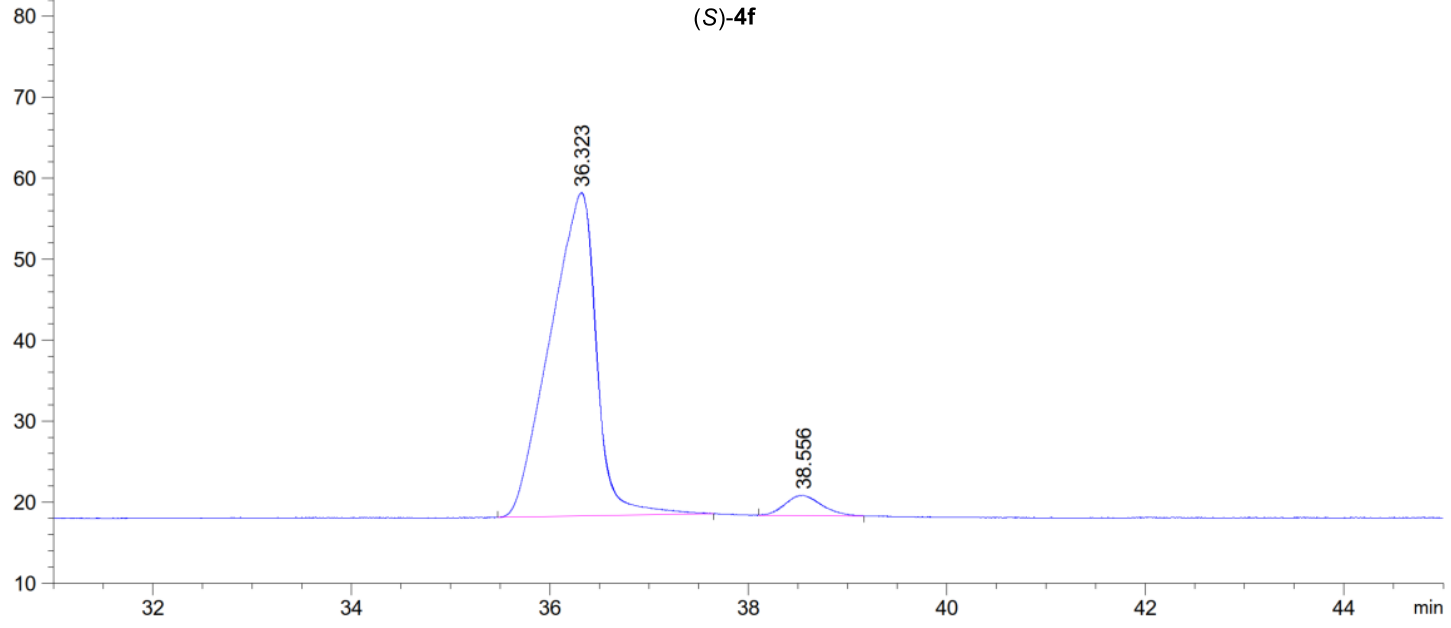

峰 保留时间 类型 峰宽 峰面积峰高 峰面积

\begin{tabular}{cccccc}
$\# \quad[\mathrm{~min}]$ & {$[\mathrm{min}]$} & {$[\mathrm{pA}$ *s $]$} & {$[\mathrm{pA}]$} & $\%$ \\
\hline 1 & 36.323 BB & 0.3862 & 1295.93359 & 39.98034 & 95.21904 \\
2 & 38.556 MM & 0.4315 & 65.06893 & 2.51346 & 4.78096
\end{tabular}




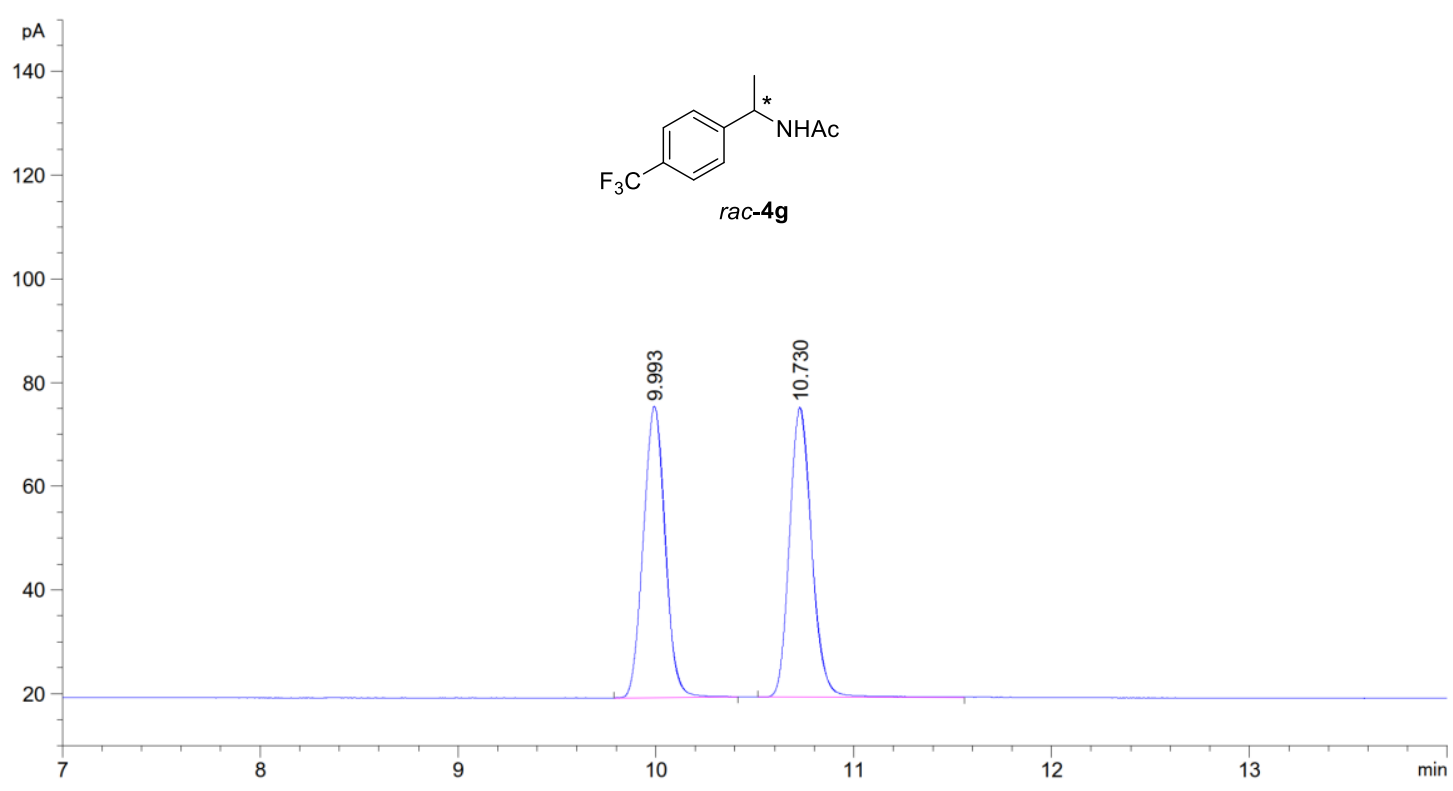

\begin{tabular}{|c|c|c|c|c|c|c|}
\hline & $\begin{array}{c}\text { 呆留时间 } \\
\text { [min] }\end{array}$ & 类型 & $\begin{array}{l}\text { 峰宽 } \\
\text { [min] }\end{array}$ & $\begin{array}{l}\text { 峰面积 } \\
{\left[\mathrm{pA} *_{\mathrm{s}}\right]}\end{array}$ & $\begin{array}{c}\text { 峰高 } \\
{[\mathrm{pA}]}\end{array}$ & $\begin{array}{c}\text { 峰面积 } \\
\%\end{array}$ \\
\hline & & & 20 & 43 & 284 & 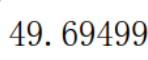 \\
\hline & 10.730 & & 0.1215 & 436. 66708 & 55.91251 & 50. 30501 \\
\hline
\end{tabular}

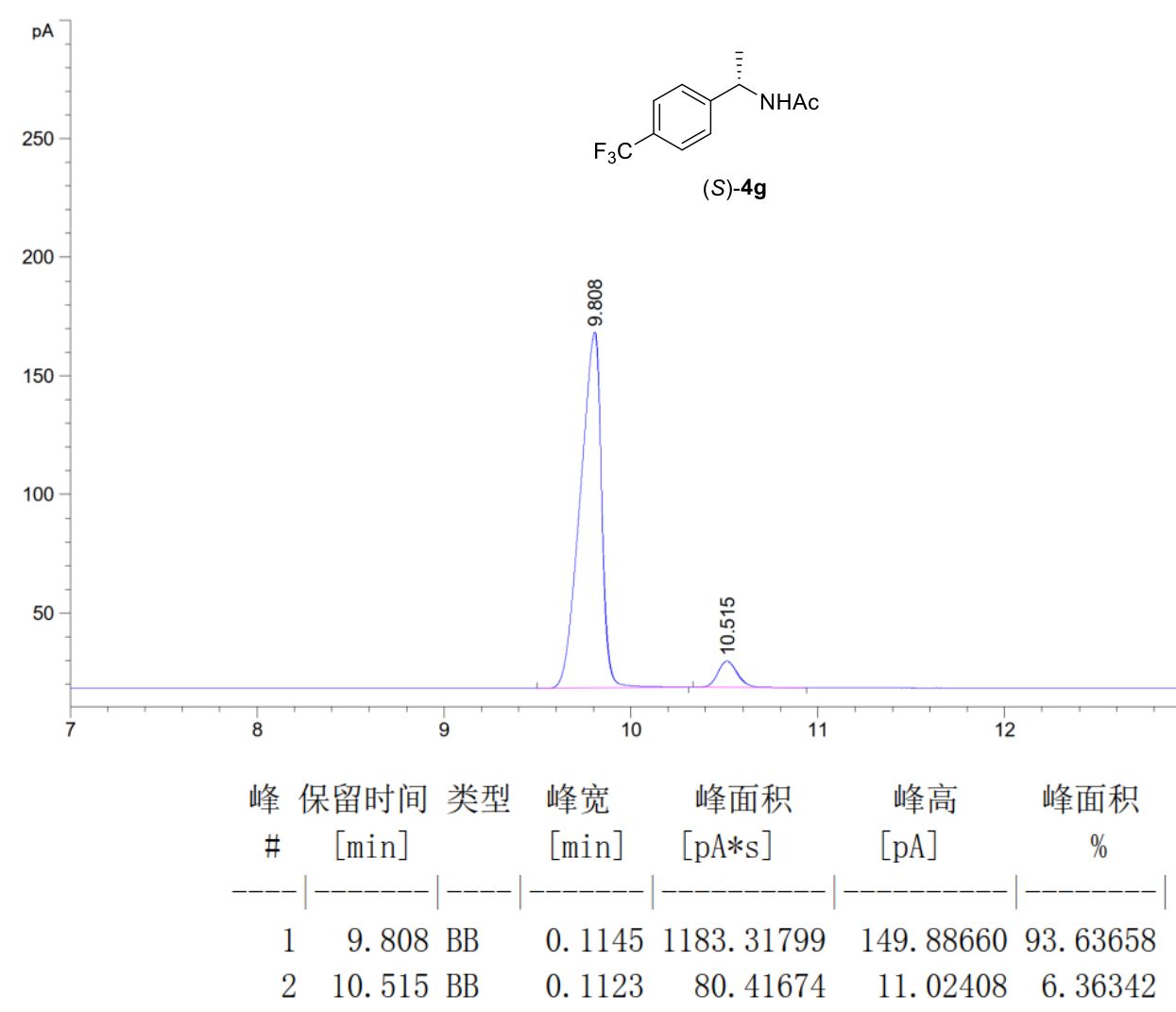




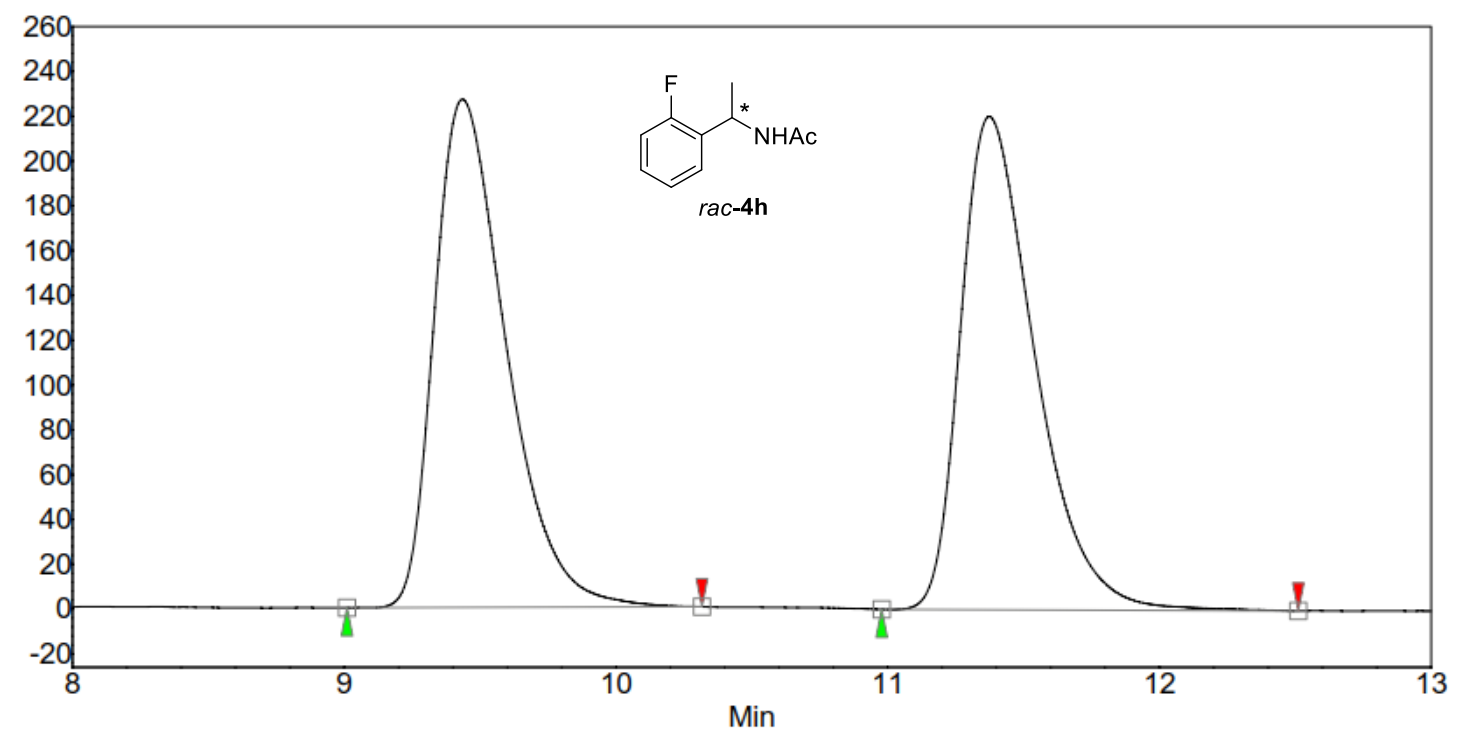

\begin{tabular}{|c|l|r|r|r|r|r|}
\hline Index & 文件名 & $\begin{array}{r}\text { 时间 } \\
\text { [Min] }\end{array}$ & $\begin{array}{r}\text { 数量 } \\
\text { [\% 面积] }\end{array}$ & $\begin{array}{r}\text { 高度 } \\
{[\mathrm{mAU}]}\end{array}$ & $\begin{array}{r}\text { Area } \\
\text { [mAU.Min] }\end{array}$ & $\begin{array}{r}\text { Area \% } \\
{[\%]}\end{array}$ \\
\hline \hline 1 & 未知 & 9.43 & 49.79 & 226.9 & 69.4 & 49.793 \\
\hline 2 & 未知 & 11.37 & 50.21 & 220.3 & 70.0 & 50.207 \\
\hline & & & & & & \\
\hline Total & & & 100.00 & 447.1 & 139.4 & 100.000 \\
\hline
\end{tabular}

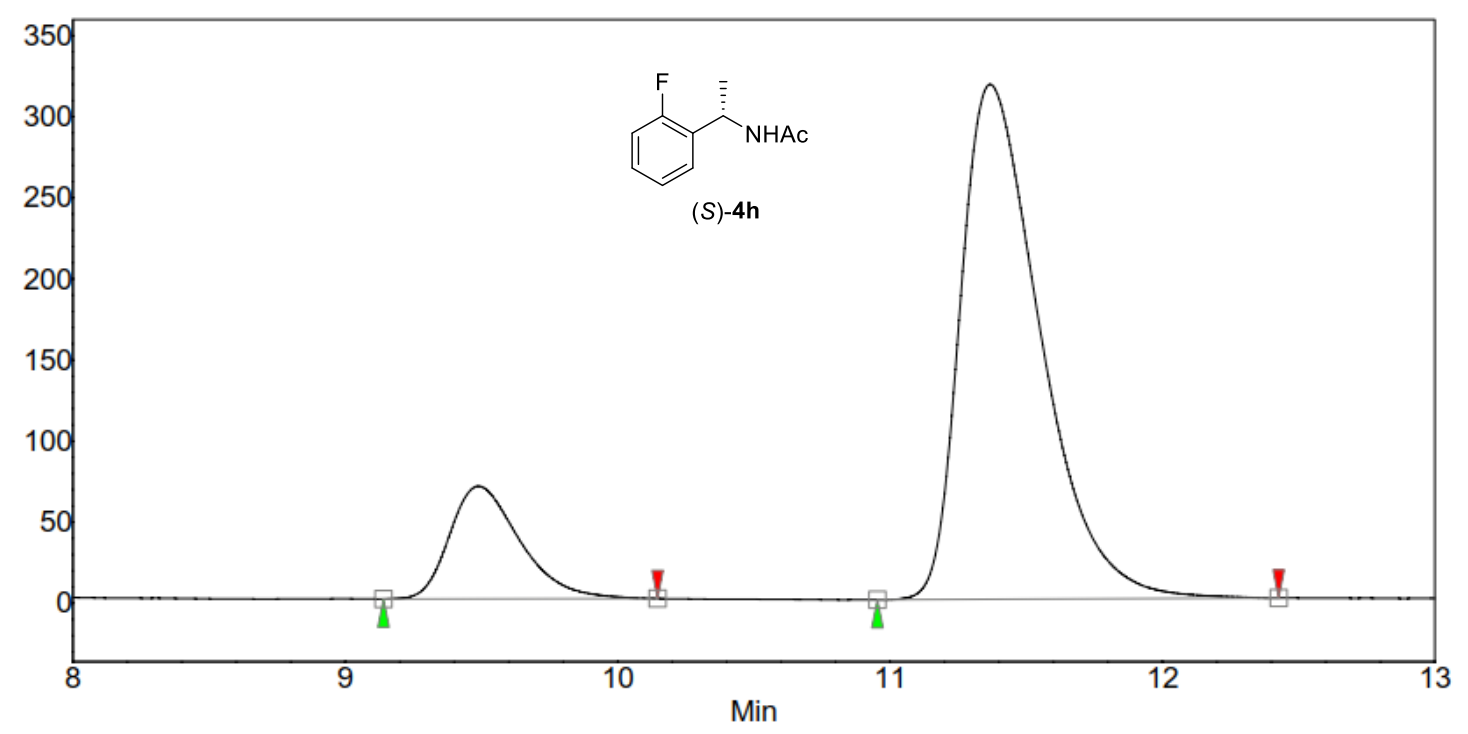

\begin{tabular}{|c|l|r|r|r|r|r|}
\hline Index & 文件名 & $\begin{array}{r}\text { 时间 } \\
\text { [Min] }\end{array}$ & $\begin{array}{r}\text { 数量 } \\
\text { [\% 面积] }\end{array}$ & $\begin{array}{r}\text { 高度 } \\
\text { [mAU] }\end{array}$ & $\begin{array}{r}\text { Area } \\
\text { [mAU.Min] }\end{array}$ & $\begin{array}{r}\text { Area \% } \\
{[\%]}\end{array}$ \\
\hline \hline 1 & 未知 & 9.49 & 16.08 & 69.4 & 20.7 & 16.084 \\
\hline 2 & 未知 & 11.37 & 83.92 & 317.6 & 108.1 & 83.916 \\
\hline & & & & & & \\
\hline Total & & & 100.00 & 387.0 & 128.8 & 100.000 \\
\hline
\end{tabular}



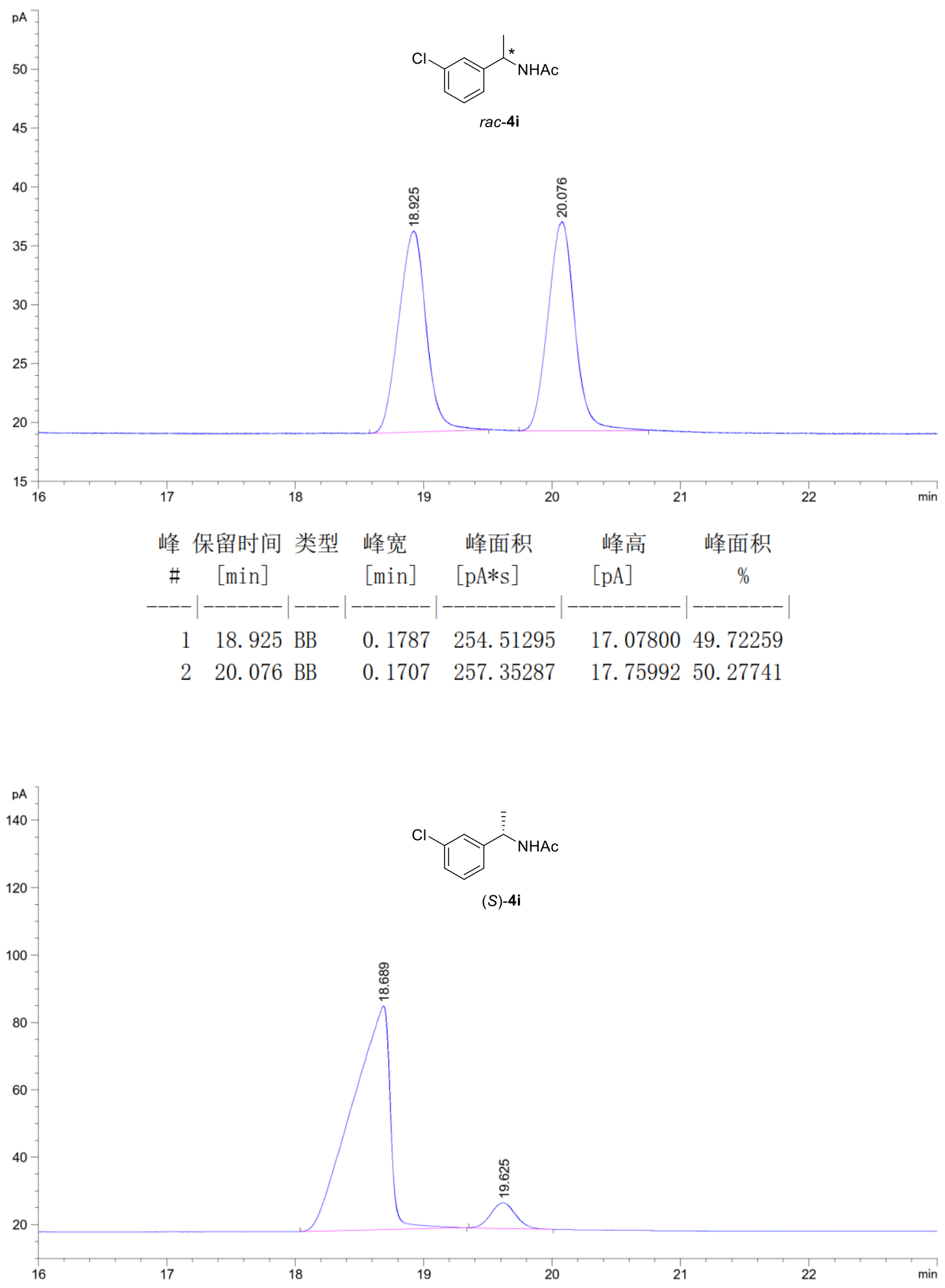

\begin{tabular}{|c|c|c|c|c|c|c|}
\hline 峰 & $\begin{array}{c}\text { 保留时间 } \\
\text { [min] }\end{array}$ & 类型 & $\begin{array}{l}\text { 峰宽 } \\
\text { [min] }\end{array}$ & $\begin{array}{c}\text { 峰面积 } \\
\text { [pA*s] }\end{array}$ & $\begin{array}{c}\text { 峰高 } \\
{[\mathrm{pA}]}\end{array}$ & $\begin{array}{c}\text { 峰面积 } \\
\%\end{array}$ \\
\hline & & & 0.2603 & 1367 & 161 & 98 \\
\hline & 19. 625 & & 0.2246 & 102.93468 & 7. 63971 & 7. 001 \\
\hline
\end{tabular}



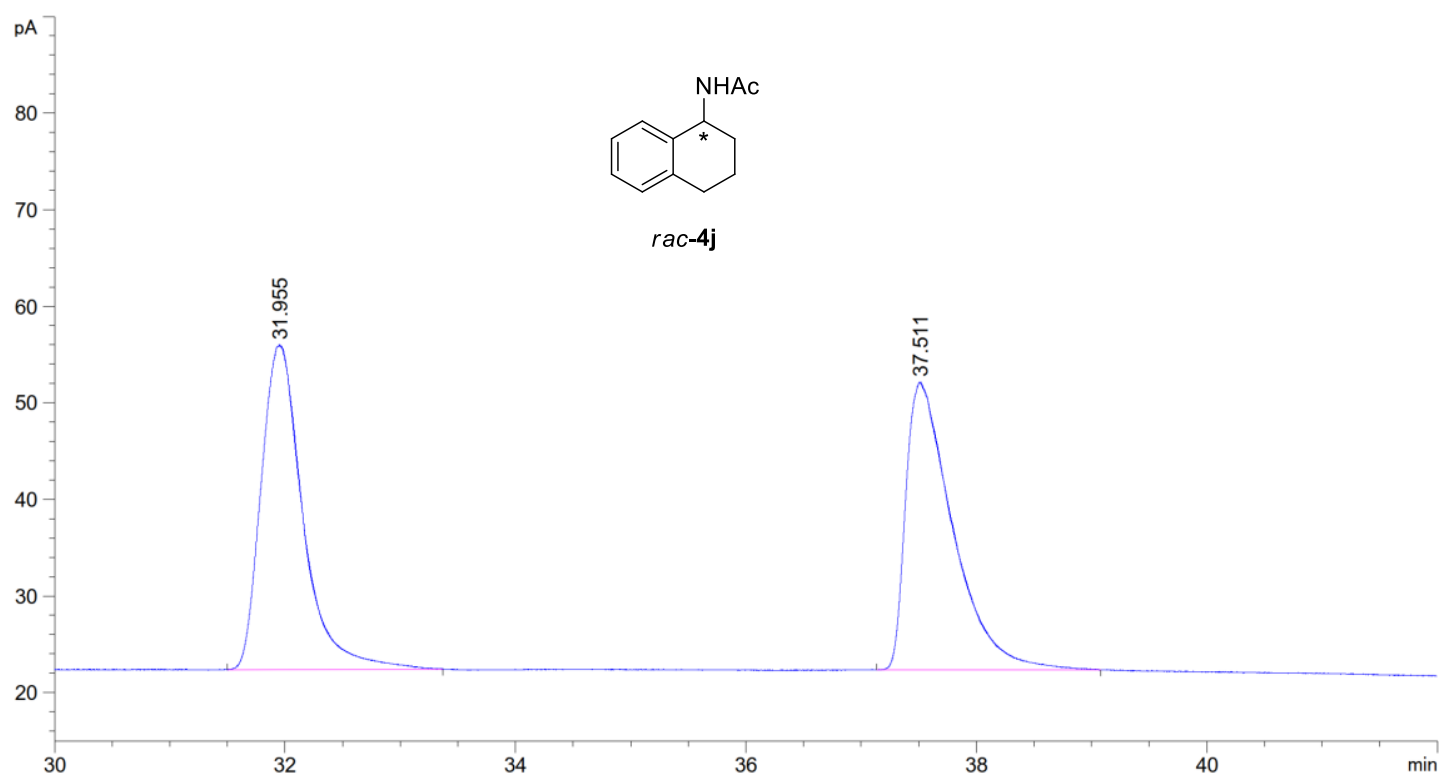

峰 保留时间 类型 峰宽峰面积峰高峰面积

$\begin{array}{cccccc}\# & {[\mathrm{~min}]} & {[\mathrm{min}]} & {\left[\mathrm{pA} *_{\mathrm{s}}\right]} & {[\mathrm{pA}]} & \% \\ -1 & 31.955 \text { BB } & 0.2963 & 849.67145 & 33.63360 & 50.12179 \\ 2 & 37.511 \text { BB } & 0.3342 & 845.54224 & 29.80530 & 49.87821\end{array}$
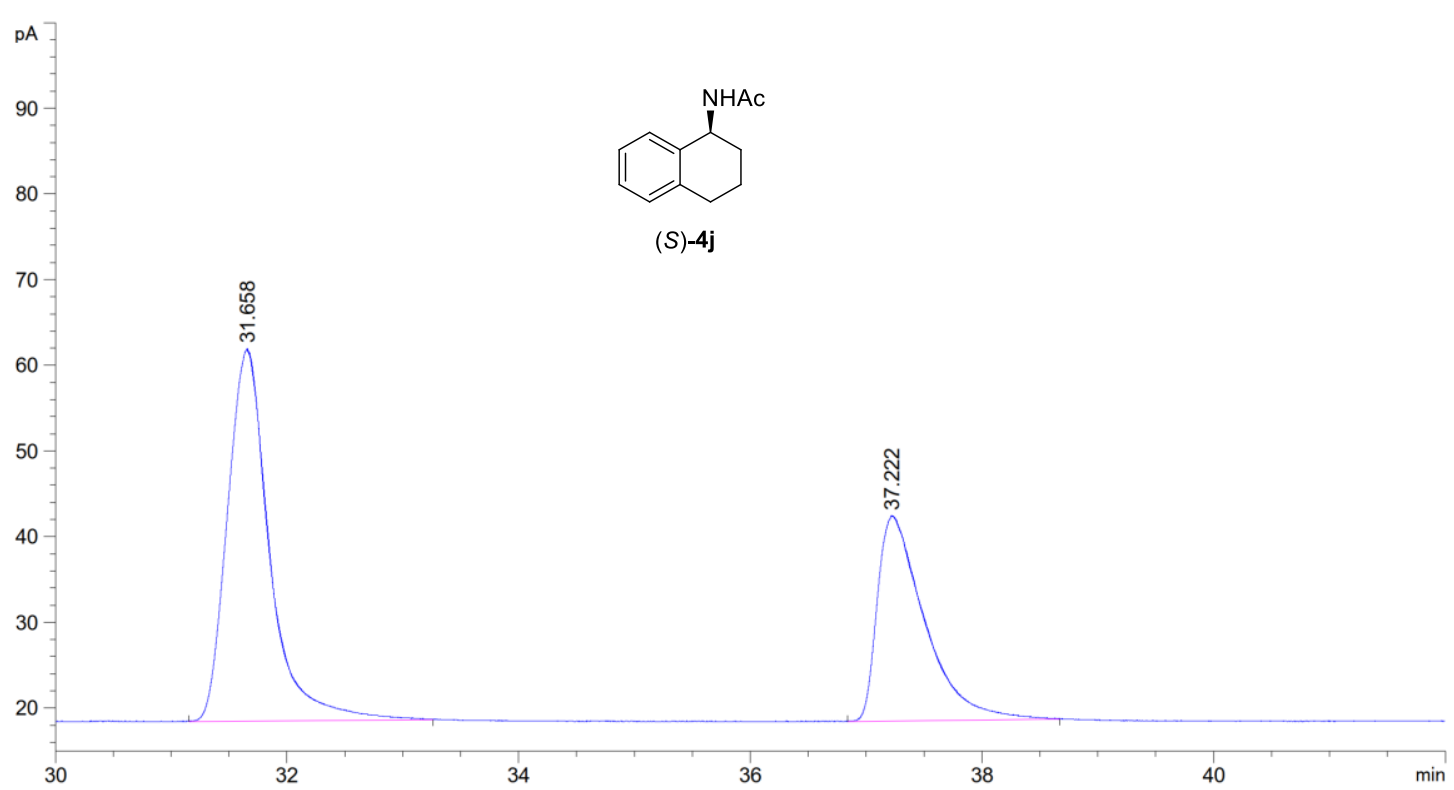

峰 保留时间 类型 峰宽 峰面积峰高峰面积

$\begin{array}{cccccc}\# & {[\mathrm{~min}]} & {[\mathrm{min}]} & {[\mathrm{pA} * \mathrm{~s}]} & {[\mathrm{pA}]} & \% \\ 1 & 31.658 \text { BB } & 0.2970 & 1093.72742 & 43.43515 & 61.67457 \\ 2 & 37.222 \mathrm{BB} & 0.3343 & 679.65723 & 23.95169 & 38.32543\end{array}$


Asymmetric hydroformylation of styrenes using $(S, S)$-L3/NaBArF

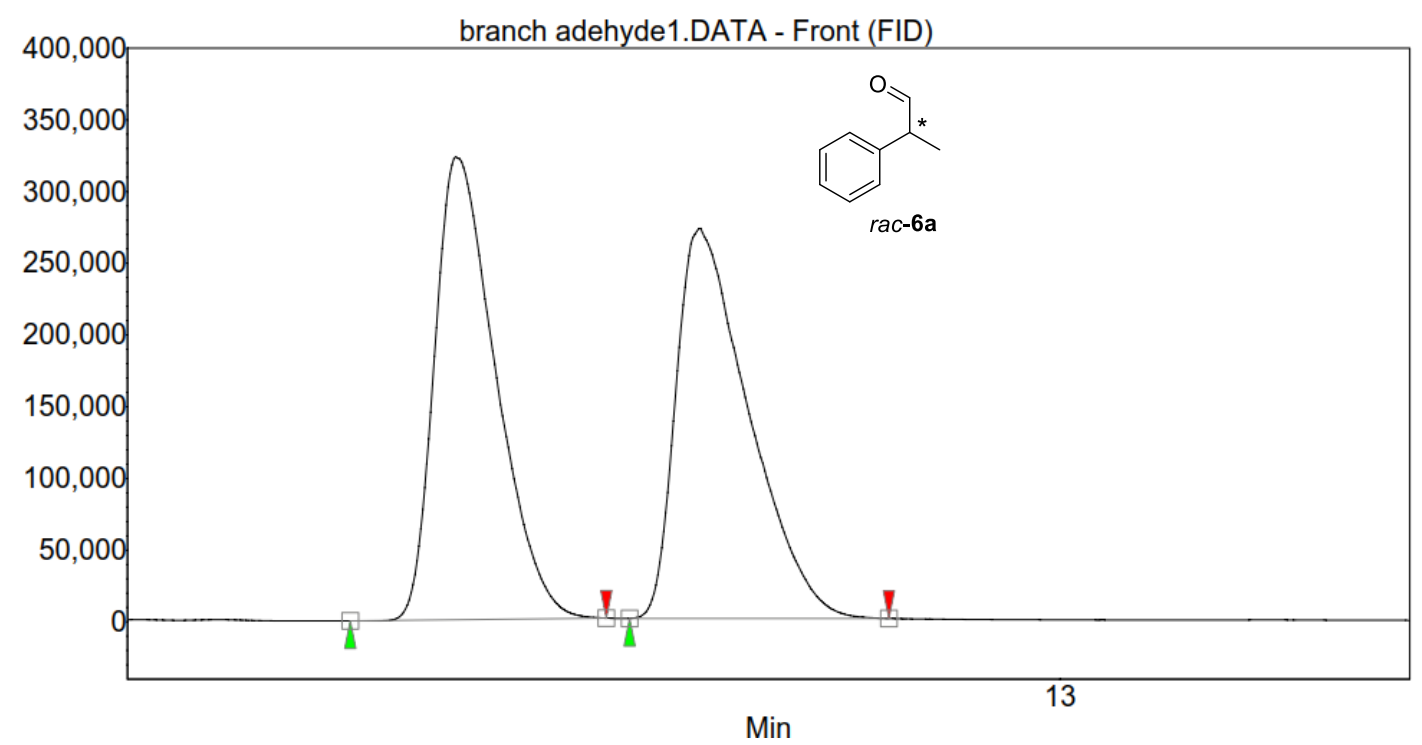

Peak results :

\begin{tabular}{|c|c|c|c|c|c|c|}
\hline Index & Name & $\begin{array}{l}\text { Time } \\
\text { [Min] }\end{array}$ & $\begin{array}{l}\text { Quantity } \\
\text { [\% Area] }\end{array}$ & $\begin{array}{r}\text { Height } \\
\text { [磺] }\end{array}$ & $\begin{array}{r}\text { Area } \\
\text { [礓.Min] }\end{array}$ & $\begin{array}{r}\text { Area \% } \\
{[\%]}\end{array}$ \\
\hline 1 & $\overline{\mathrm{UU}}$ & 12.48 & 499.99 & 322903.2 & 19617.9 & 49.994 \\
\hline 2 & UNKNOWN & 12.69 & 50.01 & 272029.5 & 19622.3 & 50.006 \\
\hline Total & & & 10000 & 5949327 & 392402 & \\
\hline
\end{tabular}

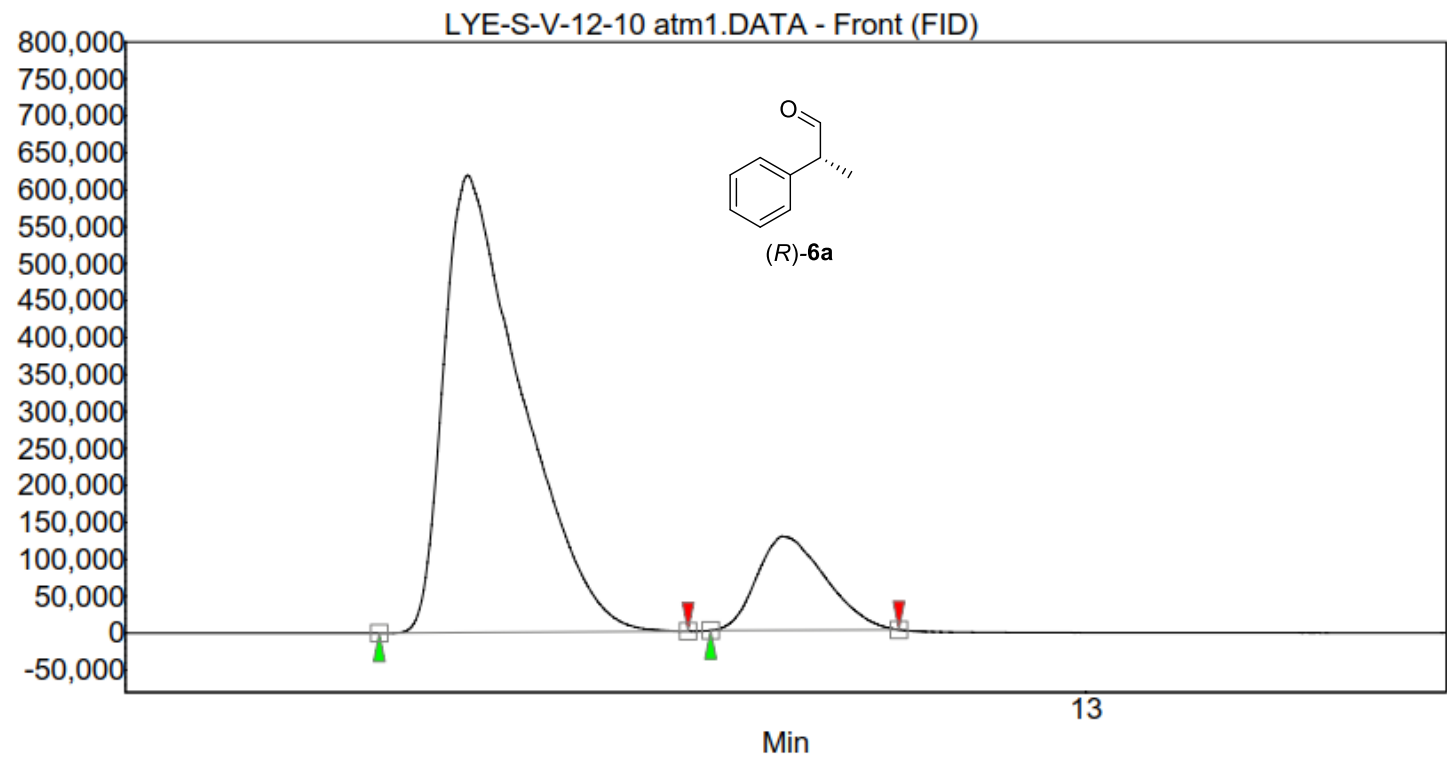

Peak results :

\begin{tabular}{|c|c|c|c|c|c|c|}
\hline Index & Name & $\begin{array}{l}\text { Time } \\
\text { [Min] }\end{array}$ & $\begin{array}{l}\text { Quantity } \\
\text { [\% 面积] }\end{array}$ & $\begin{array}{r}\text { Height } \\
\text { [磰] }\end{array}$ & 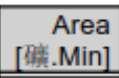 & $\begin{array}{r}\text { Area \% } \\
{[\%]} \\
\end{array}$ \\
\hline 1 & UNK & 12.49 & 84.75 & \begin{tabular}{|l}
618930.2 \\
\end{tabular} & \begin{tabular}{|l|}
46609.4 \\
\end{tabular} & 84.755 \\
\hline 2 & UNKNOWN & 12.75 & 15.25 & 127048.1 & 8383.8 & 15.245 \\
\hline otal & & & 100.00 & 745978.3 & 3.2 & 100.000 \\
\hline
\end{tabular}

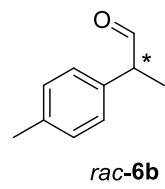




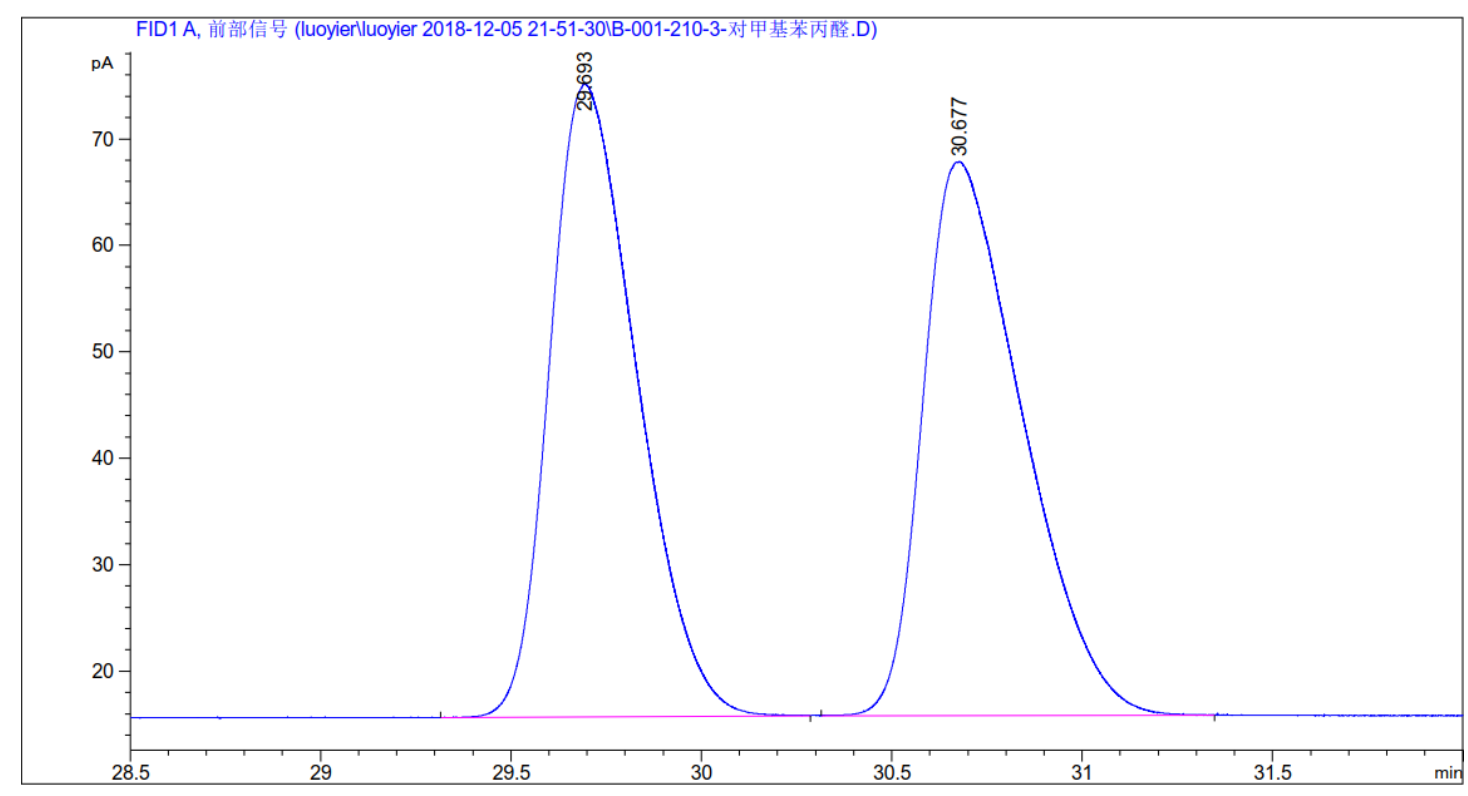

\begin{tabular}{|c|c|c|c|c|c|c|}
\hline $\begin{array}{l}\text { 峰 } \\
\#\end{array}$ & $\begin{array}{c}\text { 保留时间 } \\
{[\text { min] }}\end{array}$ & 类型 & $\begin{array}{l}\text { 峰宽 } \\
\text { [min] }\end{array}$ & $\begin{array}{c}\text { 峰面积 } \\
\text { [pA*s] }\end{array}$ & $\begin{array}{c}\text { 峰高 } \\
{[\mathrm{pA}]}\end{array}$ & $\begin{array}{c}\text { 峰面积 } \\
\%\end{array}$ \\
\hline 1 & 29 & & 0.2100 & 943.89545 & 59. 47013 & 50.05560 \\
\hline 2 & 30.677 & D & 0.2273 & 941.79871 & 52.00474 & 49.94440 \\
\hline
\end{tabular}

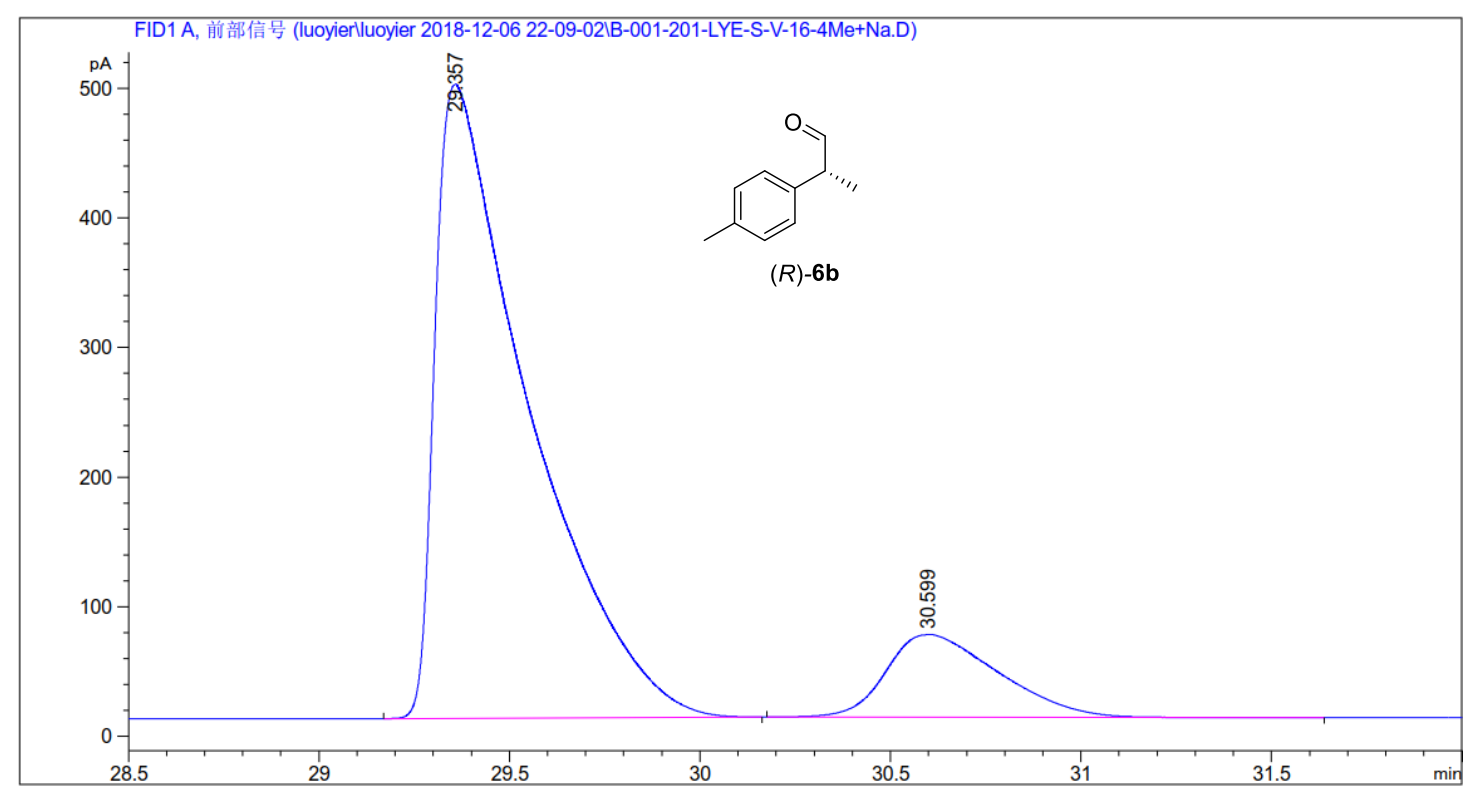

峰 保留时间 类型 峰宽 峰面积峰高峰面积

\begin{tabular}{|c|c|c|c|c|c|}
\hline \# & [min] & {$[\mathrm{min}]$} & {$[\mathrm{pA} * \mathrm{~s}]$} & {$[\mathrm{pA}]$} & $\%$ \\
\hline 1 & 29. $357 \mathrm{BB}$ & 0.2269 & 8344.57715 & 489. 38519 & 86.45770 \\
\hline 2 & $30.599 \mathrm{BB}$ & 0.2881 & 1307. 05212 & 63.71766 & 13. 54230 \\
\hline
\end{tabular}




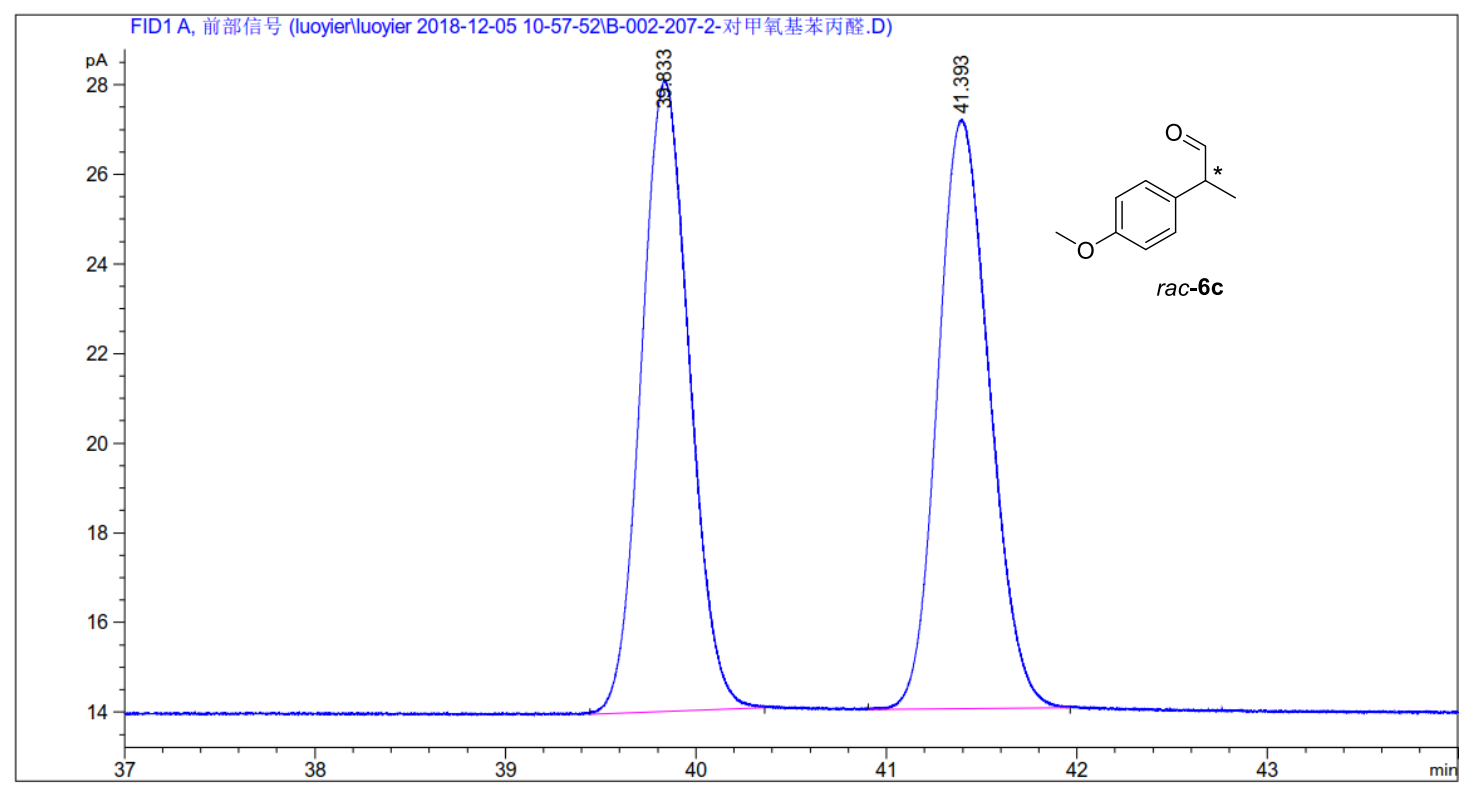

\begin{tabular}{|c|c|c|c|c|c|c|}
\hline $\begin{array}{r}\text { 峰 } \\
\text { \# }\end{array}$ & $\begin{array}{c}\text { 保留时间 } \\
\text { [min] }\end{array}$ & 类型 & $\begin{array}{l}\text { 峰宽 } \\
\text { [min] }\end{array}$ & $\begin{array}{c}\begin{array}{c}\text { 峰面积 } \\
\text { [pA*s }]\end{array}\end{array}$ & $\begin{array}{c}\begin{array}{l}\text { 峰高 } \\
{[\mathrm{pA}]}\end{array}\end{array}$ & $\begin{array}{c}\text { 峰面积 } \\
\%\end{array}$ \\
\hline 1 & 39.833 & BB & 0.2138 & 247. 16164 & 14. 06544 & 49. 82913 \\
\hline 2 & 41. 393 & B & 0.2344 & 248.85672 & 13. 15009 & 50.17087 \\
\hline
\end{tabular}

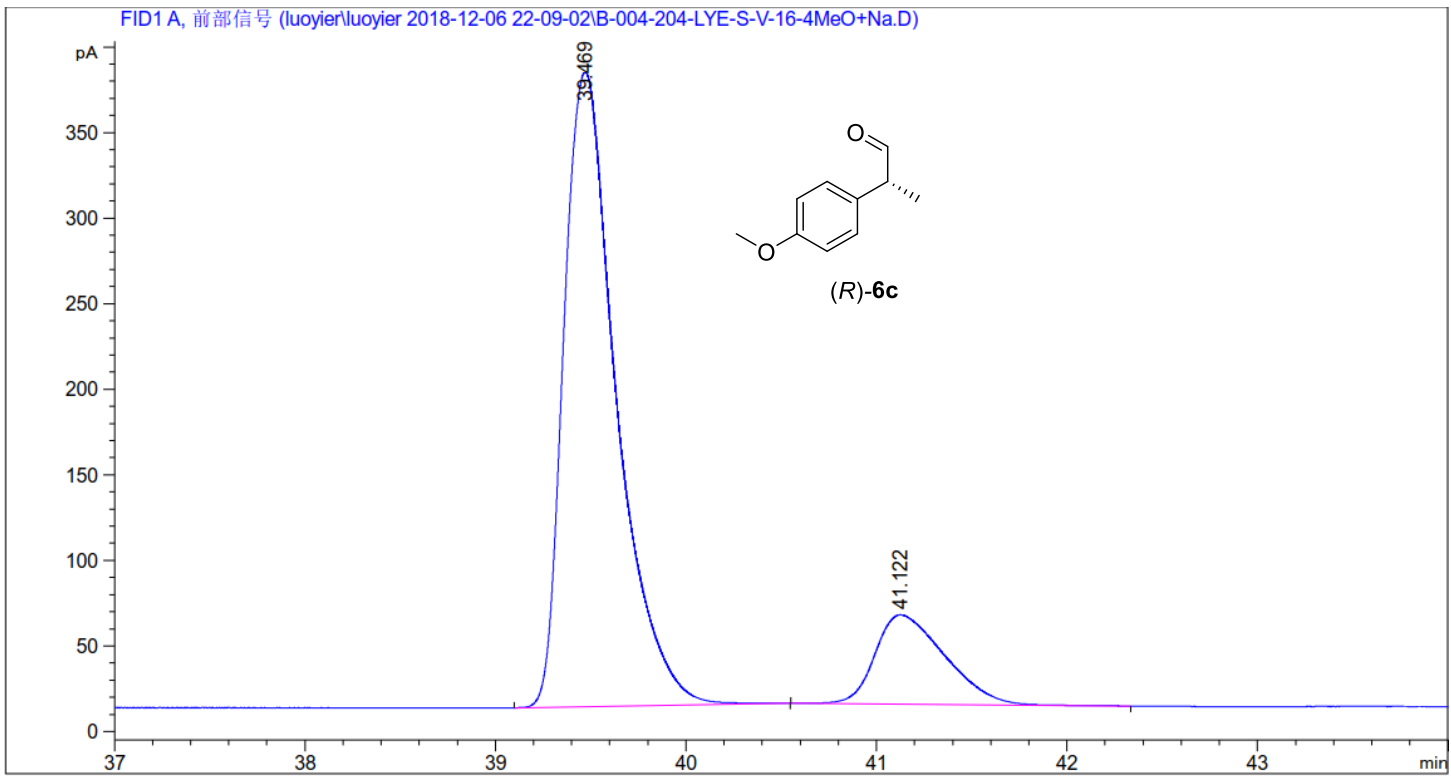

\begin{tabular}{|c|c|c|c|c|c|c|}
\hline & $\begin{array}{c}\text { 保留时间 } \\
\text { [min] }\end{array}$ & 类型 & $\begin{array}{l}\text { 峰宽 } \\
\text { [min] }\end{array}$ & $\begin{array}{l}\text { 峰面积 } \\
{[\mathrm{pA*s} \text { ) }}\end{array}$ & $\begin{array}{l}\begin{array}{l}\text { 峰高 } \\
{[\mathrm{pA}]}\end{array}\end{array}$ & $\begin{array}{c}\text { 峰面积 } \\
\%\end{array}$ \\
\hline & 39. $46 \mathrm{c}$ & & 2750 & 7079. 87598 & 370.43759 & 4814 \\
\hline & 41.122 & & 0.3378 & 1333. 70972 & 52.38346 & 15. 85186 \\
\hline
\end{tabular}




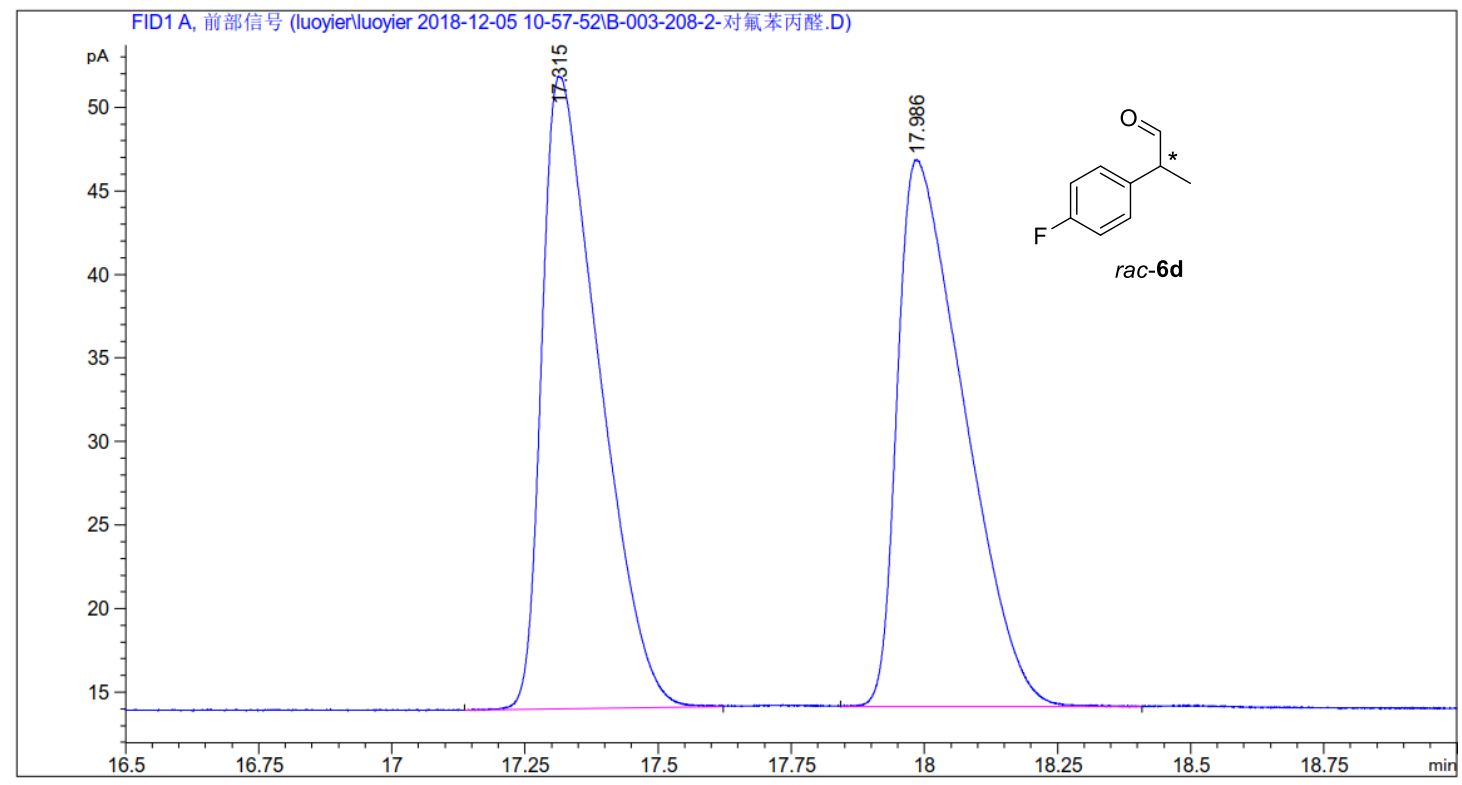

峰 保留时间 类型 峰宽峰面积峰高峰面积

\begin{tabular}{|c|c|c|c|c|c|}
\hline \# & {$[\min ]$} & [min] & {$[\mathrm{pA} * \mathrm{~s}]$} & {$[\mathrm{pA}]$} & $\%$ \\
\hline 1 & 17. $315 \mathrm{BB}$ & 0.1073 & 282. 76889 & 37.81016 & 49.91328 \\
\hline 2 & 17. $986 \mathrm{BB}$ & 0.1292 & 283. 75143 & 32.70085 & 50.08672 \\
\hline
\end{tabular}

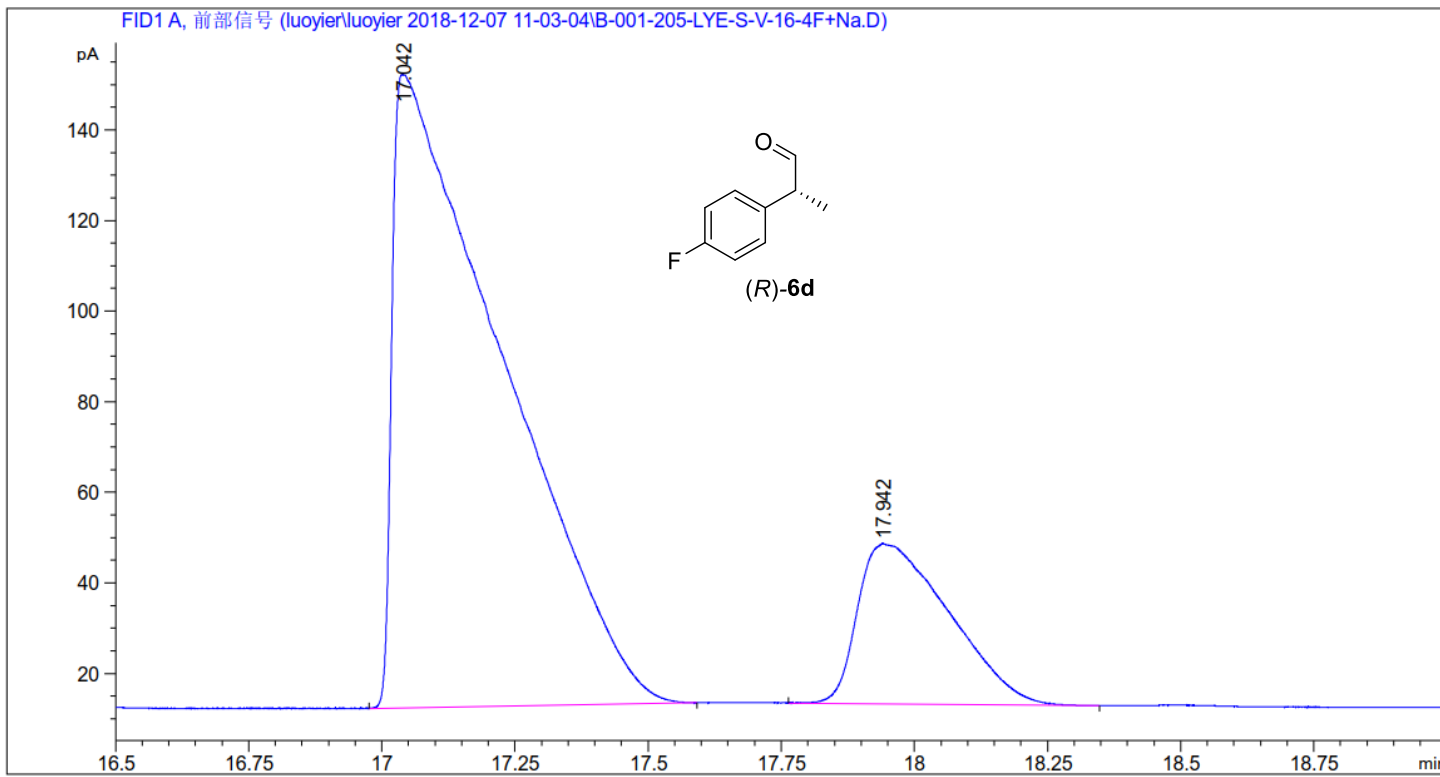

\begin{tabular}{|c|c|c|c|c|c|c|}
\hline $\begin{array}{l}\text { 峰 } \\
\text { \# }\end{array}$ & $\begin{array}{c}\text { 保留时间 } \\
\text { [min] }\end{array}$ & 类型 & $\begin{array}{l}\text { 峰宽 } \\
\text { [min] }\end{array}$ & $\begin{array}{l}\text { 峰面积 } \\
{[\mathrm{pA*s}]}\end{array}$ & $\begin{array}{l}\text { 峰高 } \\
{[\mathrm{pA}]}\end{array}$ & $\begin{array}{c}\text { 峰面积 } \\
\%\end{array}$ \\
\hline 1 & 17 & & 0.1832 & 2001. 72729 & 139. 67984 & .59314 \\
\hline & 17. 942 & & 0.1578 & 421.87274 & 35. 30652 & 17. 4068 \\
\hline
\end{tabular}



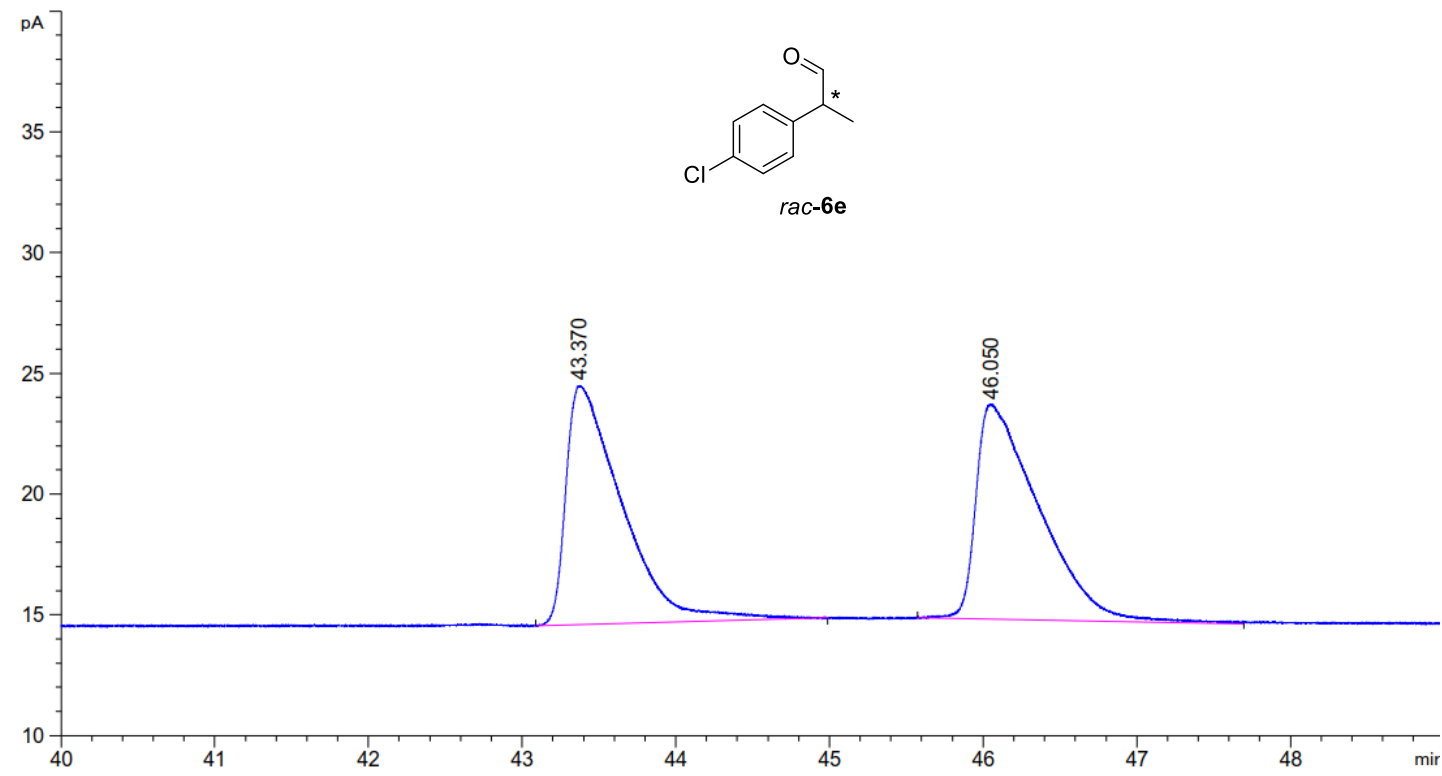

\begin{tabular}{|c|c|c|c|c|c|c|}
\hline $\begin{array}{r}\text { 峰 } \\
\text { \# }\end{array}$ & $\begin{array}{c}\text { 呆留时间 } \\
\text { [min] }\end{array}$ & 类型 & $\begin{array}{l}\text { 峰宽 } \\
\text { [min] }\end{array}$ & $\begin{array}{c}\text { 峰面积 } \\
\text { [pA*s] }\end{array}$ & $\begin{array}{l}\text { 峰高 } \\
\text { [pA] }\end{array}$ & $\begin{array}{c}\text { 峰面积 } \\
\%\end{array}$ \\
\hline 1 & 43.370 & MM & 0.4209 & 250.28178 & 9. 91026 & 49. 75095 \\
\hline 2 & 46. 050 & MM & 0.4744 & 252. 78757 & 8.88183 & 50.24905 \\
\hline
\end{tabular}

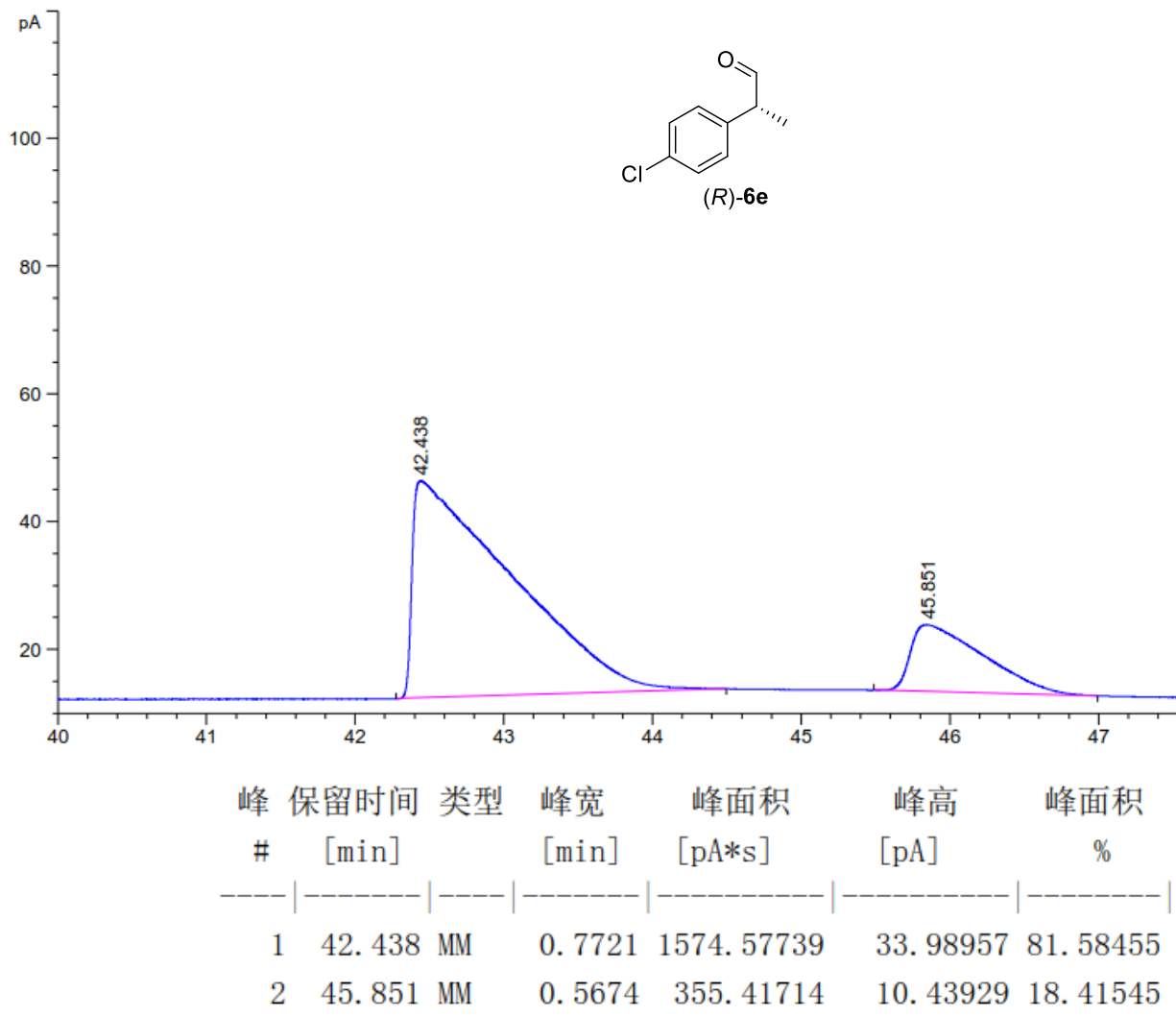




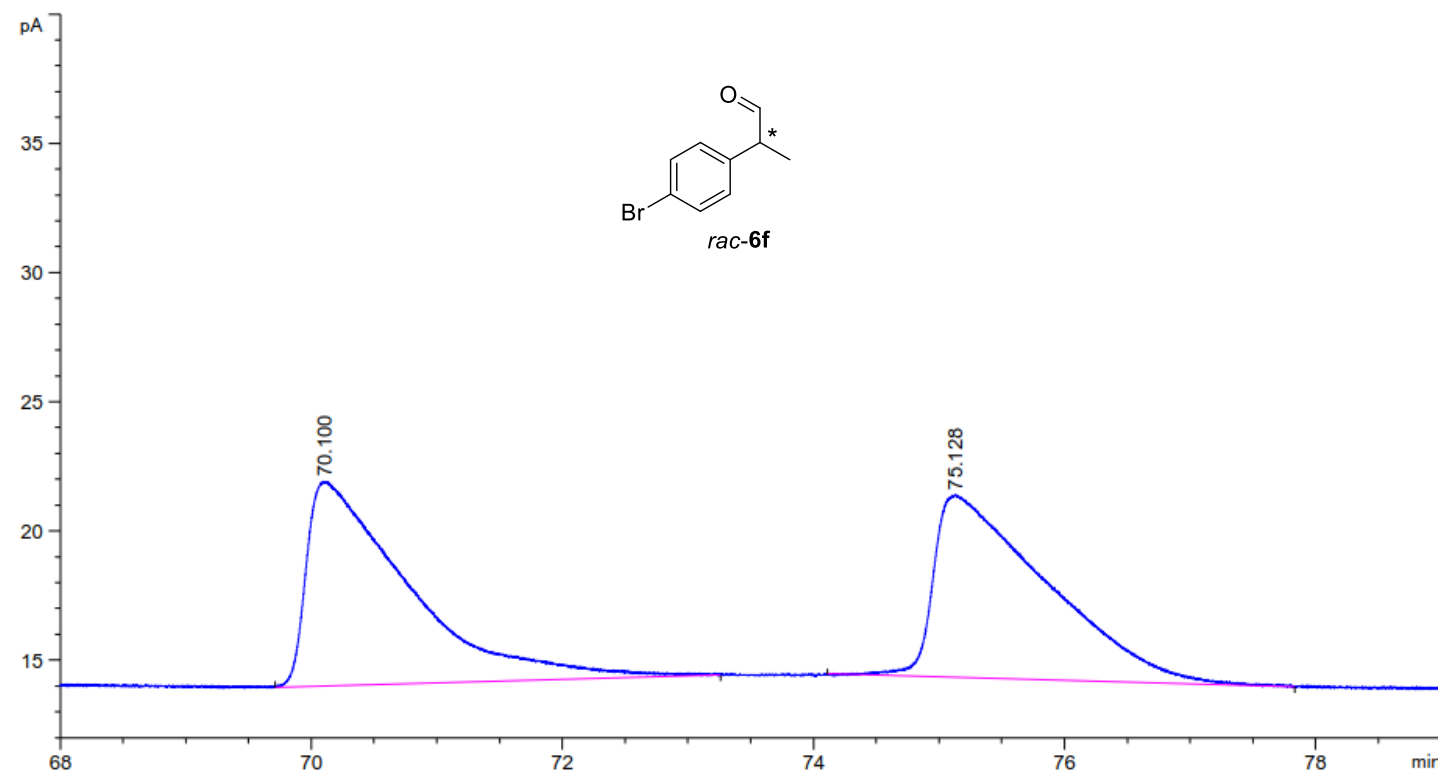

峰保留时间类型峰宽峰面积峰高峰面积

$\begin{array}{cccccc}\# & {[\mathrm{~min}]} & {[\mathrm{min}]} & {[\mathrm{pA} * \mathrm{~s}]} & {[\mathrm{pA}]} & \% \\ -1 & 70.100 \mathrm{MM} & 0.9417 & 446.65872 & 7.90533 & 50.41813 \\ 2 & 75.128 \mathrm{MM} & 1.0392 & 439.25015 & 7.04453 & 49.58187\end{array}$

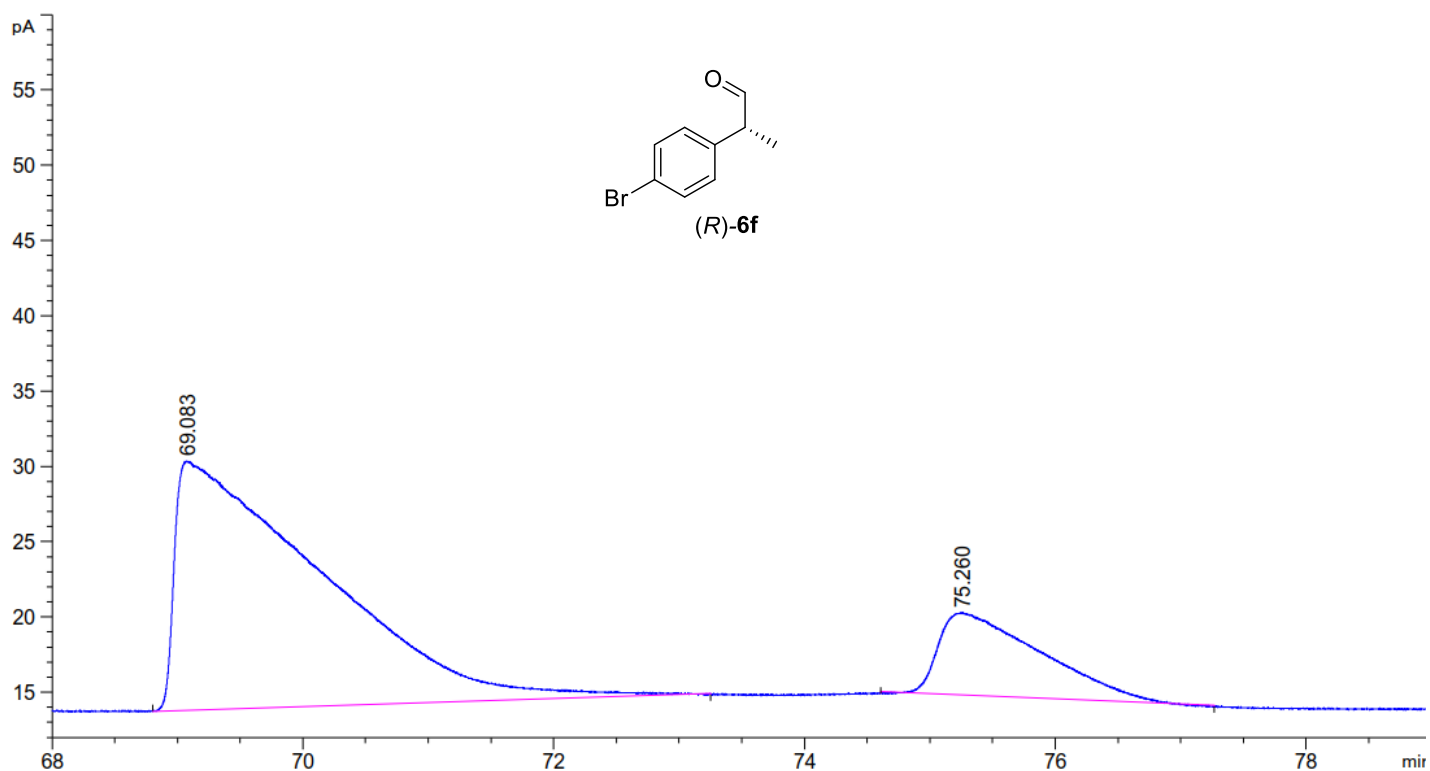

峰 保留时间 类型 峰宽 峰面积峰高 峰面积

\begin{tabular}{cccccc}
$\#$ & {$[\mathrm{~min}]$} & {$[\mathrm{min}]$} & {$[\mathrm{pA}$ *s $]$} & \multicolumn{1}{c}{ [pA] } & $\%$ \\
1 & 69.083 MM & 1.3453 & 1334.89319 & 16.53771 & 81.22497 \\
2 & 75.260 MM & 0.9509 & 308.55847 & 5.40841 & 18.77503
\end{tabular}

